Prepared for the California Department of Water Resources, Salton Sea Ecosystem Restoration Program

\title{
Salton Sea Ecosystem Monitoring and Assessment Plan
}

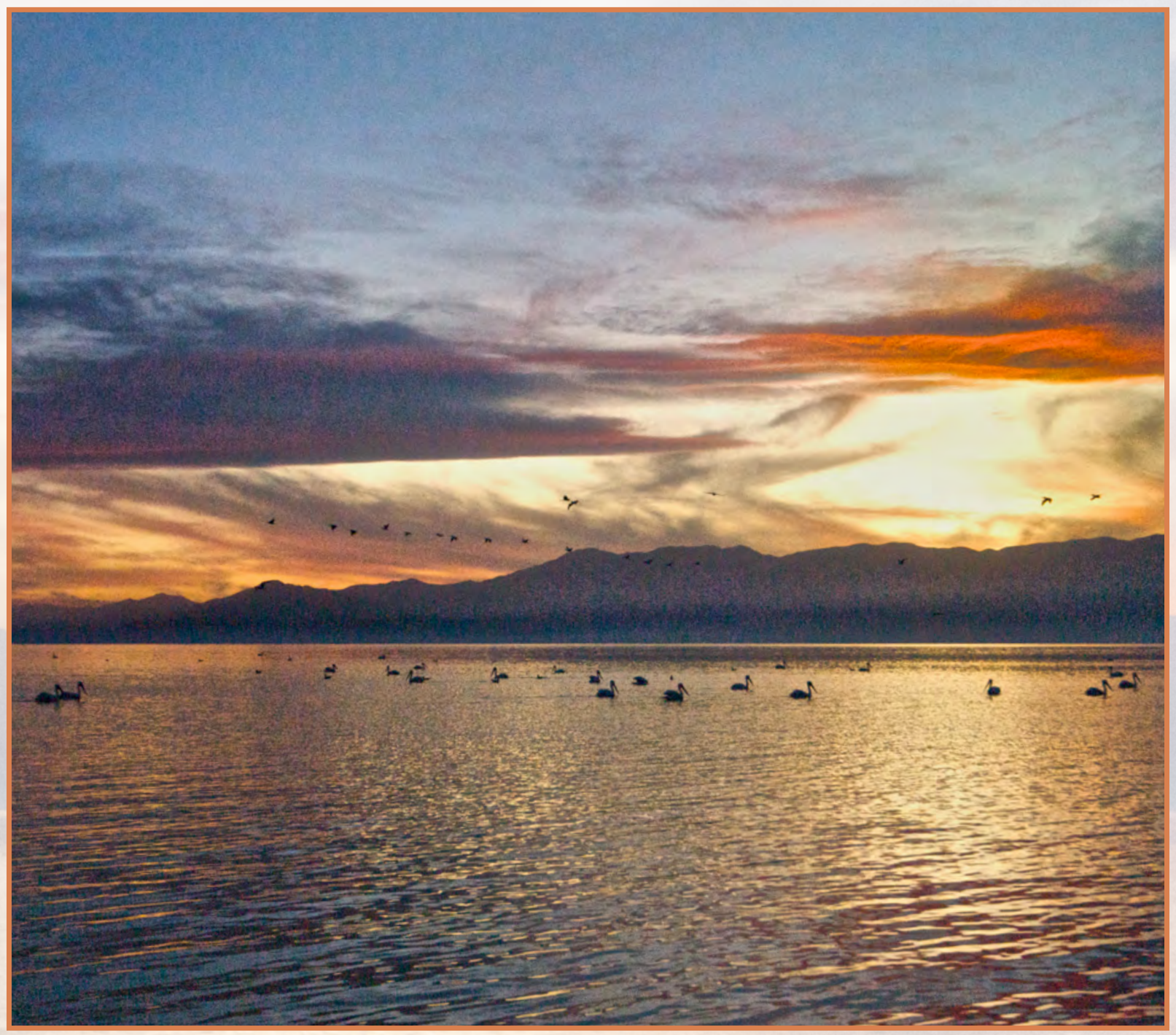

Open-File Report 2013-1133 
Cover. Photograph of Salton Sea at sunset taken by Douglas Barnum, U.S. Geological Survey. 


\section{Salton Sea Ecosystem Monitoring and Assessment Plan}

Compiled by H.L. Case III, U.S. Geological Survey; Jerry Boles, California Department of Water Resources; Arturo Delgado, California Department of Fish and Wildlife; Thang Nguyen, California Department of Water Resources; Doug Osugi, California Department of Water Resources; Douglas A. Barnum, U.S. Geological Survey; Drew Decker, U.S. Geological Survey; Steven Steinberg, Southern California Coastal Water Research Project; Sheila Steinberg, Brandman University; Charles Keene, California Department of Water Resources; Kristina White, California Department of Fish and Wildlife; Tom Lupo, California Department of Fish and Wildlife; Sheldon Gen, San Francisco State University; and Ken A. Baerenklau, University of California, Riverside

Prepared for the California Department of Water Resources, Salton Sea Ecosystem Restoration Program

Open-File Report 2013-1133

\section{U.S. Department of the Interior}

\section{U.S. Geological Survey}




\section{U.S. Department of the Interior \\ Sally Jewell, Secretary}

\section{U.S. Geological Survey \\ Suzette Kimball, Acting Director}

U.S. Geological Survey, Reston, Virginia 2013

For product and ordering information:

World Wide Web: http://www.usgs.gov/pubprod

Telephone: 1-888-ASK-USGS

For more information on the USGS-the Federal source for science about the Earth,

its natural and living resources, natural hazards, and the environment:

World Wide Web: http://www.usgs.gov

Telephone: 1-888-ASK-USGS

Suggested citation:

Case III, H.L.; Boles, Jerry; Delgado, Arturo; Nguyen, Thang; Osugi, Doug; Barnum, D.A.; Decker, Drew; Steinberg, Steven; Steinberg, Sheila; Keene, Charles; White, Kristina; Lupo, Tom; Gen, Sheldon; and Baerenklau, K.A., Salton Sea ecosystem monitoring and assessment plan: U.S. Geological Survey Open-File Report 2013-1133, 220 p.

Any use of trade, product, or firm names is for descriptive purposes only and does not imply endorsement by the U.S. Government.

Although this report is in the public domain, permission must be secured from the individual copyright owners to reproduce any copyrighted material contained within this report. 


\section{Contents}

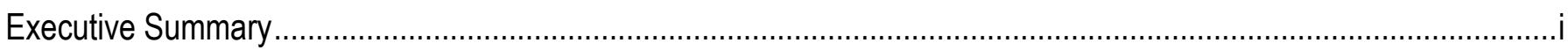

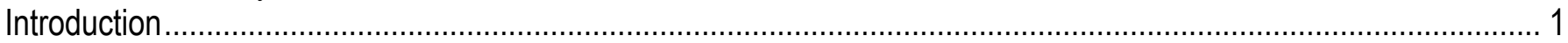

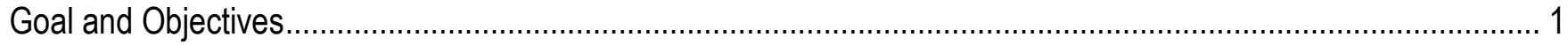

Scope

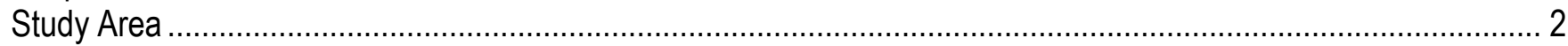

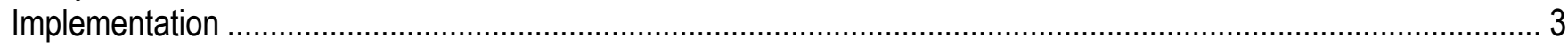

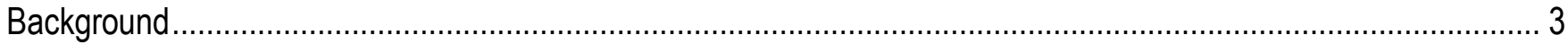

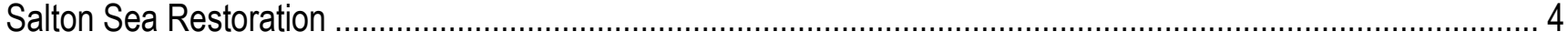

Document Organization and Intent.................................................................................................. 5

Plan Development

Focused Technical Groups..........................................................................................................

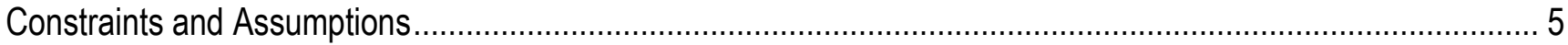

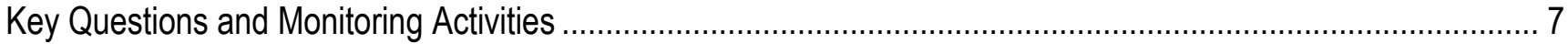

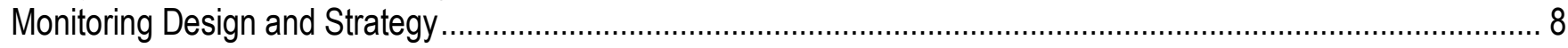

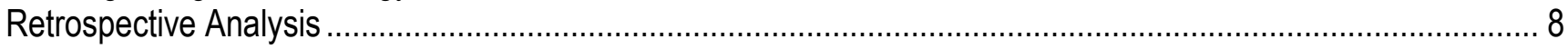

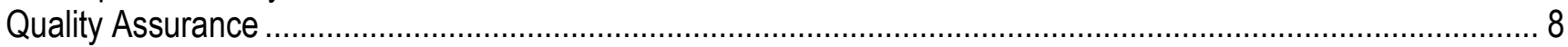

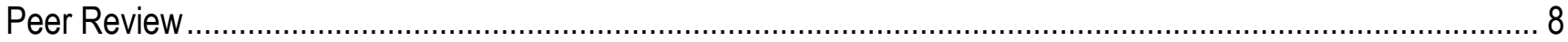

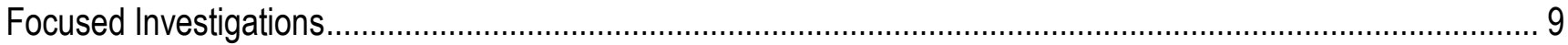

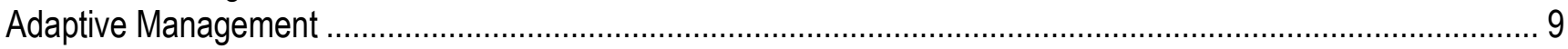

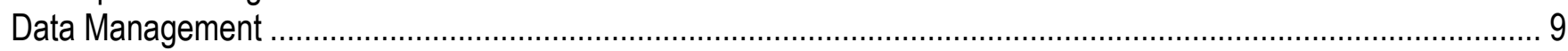

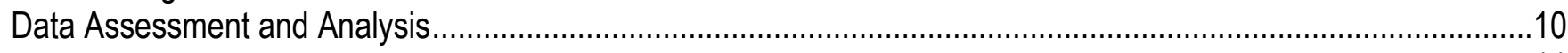

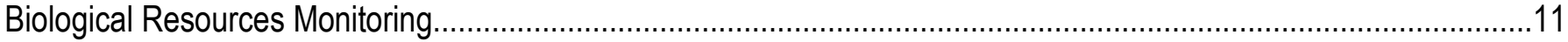

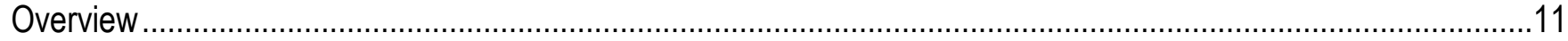

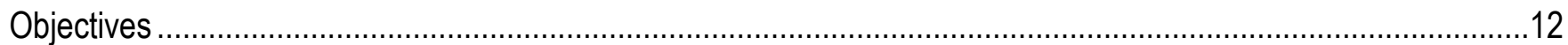

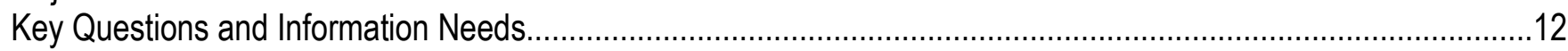

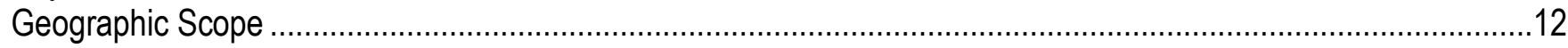

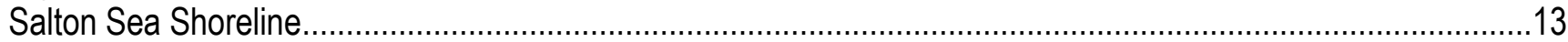

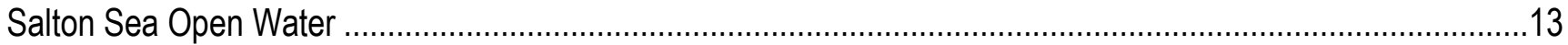

Created Freshwater Impoundments ................................................................................................

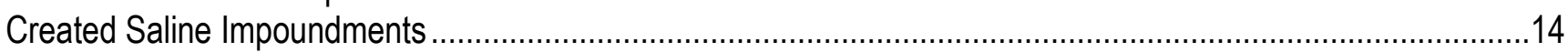

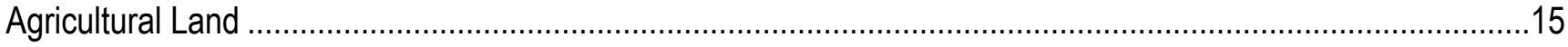

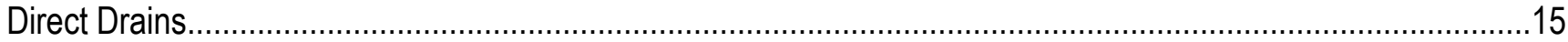

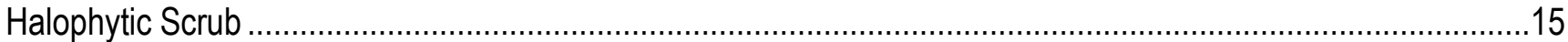

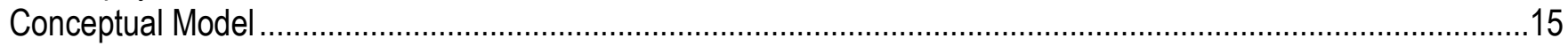

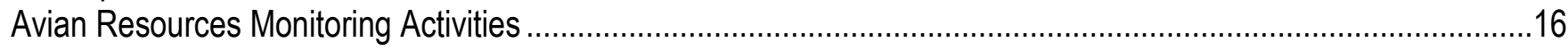

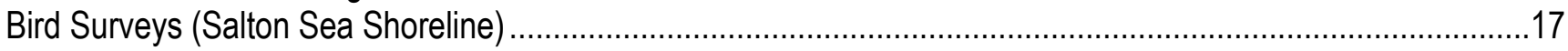

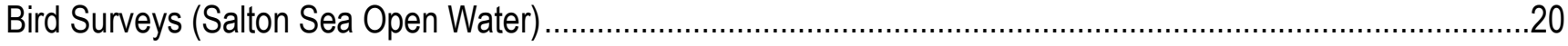

Bird Surveys (Created Saline Impoundments) ……………..................................................................22

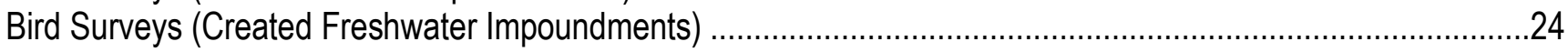

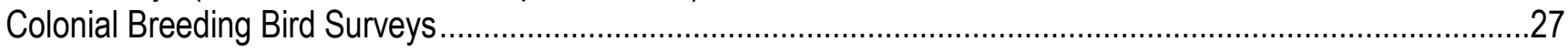

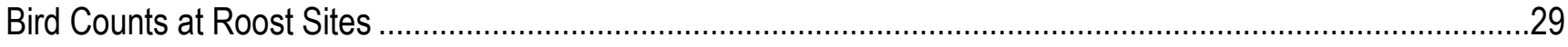

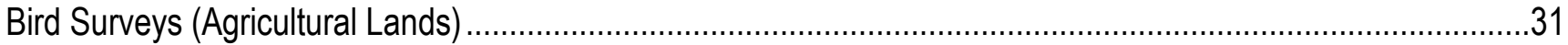

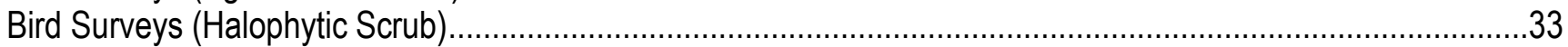

Dead and Sick Bird Counts ………………………………..................................................................

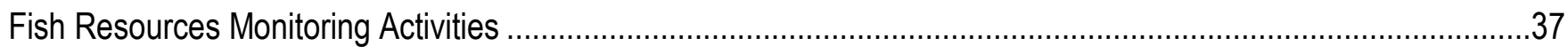




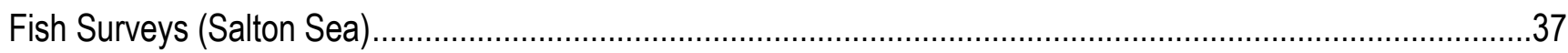

Desert Pupfish Surveys ..............................................................................................................40

Plankton and Macroinvertebrate Resources Monitoring Activities ..................................................................42

Water-Column Phytoplankton Surveys (Salton Sea) ...........................................................................4

Water-Column Phytoplankton Surveys (Created Saline Impoundments) .......................................................45

Water-Column Phytoplankton Surveys (Created Freshwater Impoundments) ................................................47

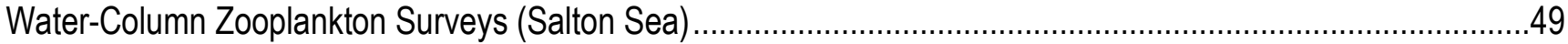

Water-Column Zooplankton Surveys (Created Saline Impoundments) .........................................................52

Water-Column Zooplankton Surveys (Created Freshwater Impoundments) .....................................................54

Water-Column Macroinvertebrate Surveys (Salton Sea) .........................................................................56

Water-Column Macroinvertebrate Surveys (Created Saline Impoundments) ...................................................58

Water-Column Macroinvertebrate Surveys (Created Freshwater Impoundments) ............................................60

Benthic Macroinvertebrate Surveys (Salton Sea) ................................................................................62

Benthic Macroinvertebrate Surveys (Created Saline Impoundments) ..............................................................65

Benthic Macroinvertebrate Surveys (Created Freshwater Impoundments) …................................................67

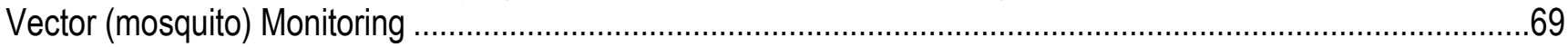

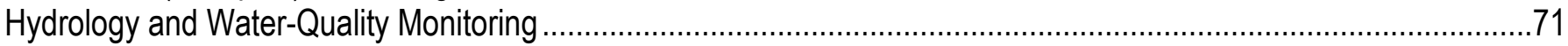

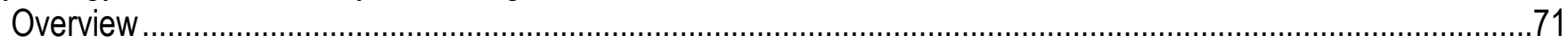

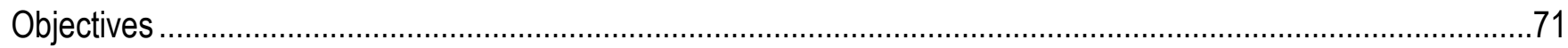

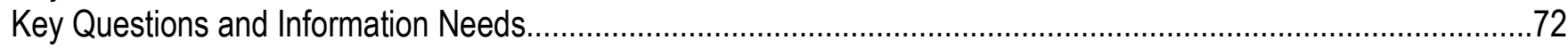

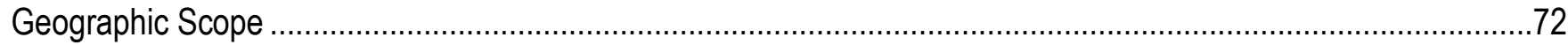

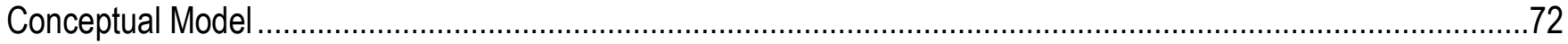

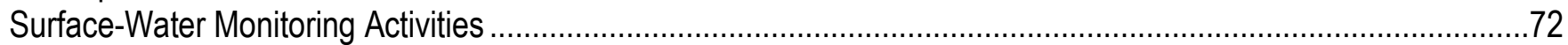

Measurement of Tributary Flows (gaging) ............................................................................................

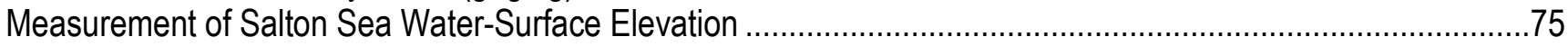

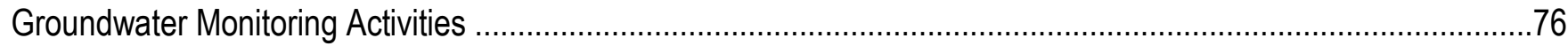

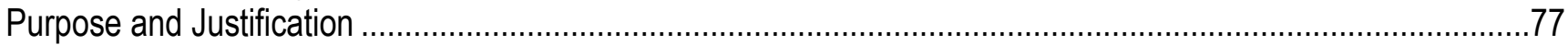

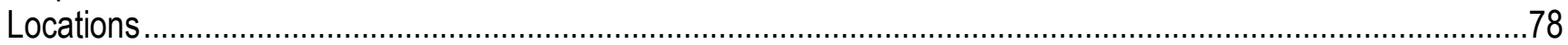

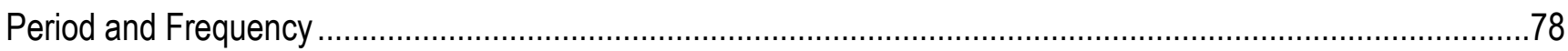

Protocol and Data Collection .............................................................................................................

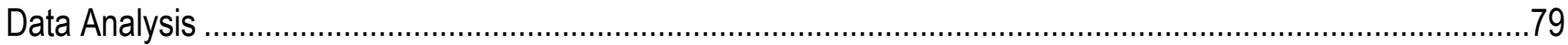

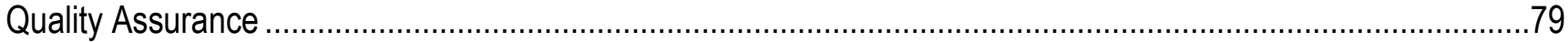

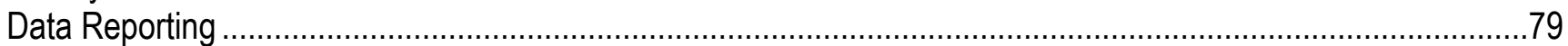

Overview of Similar Current Monitoring Activities....................................................................................8

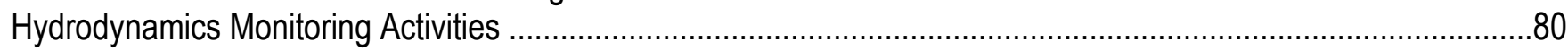

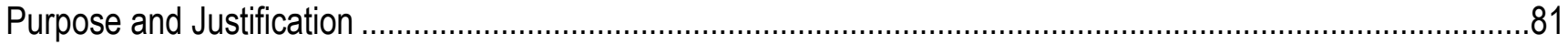

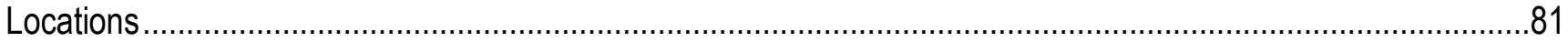

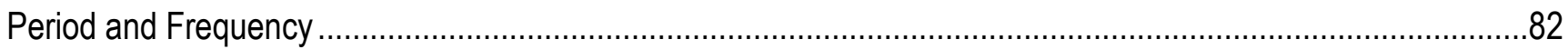

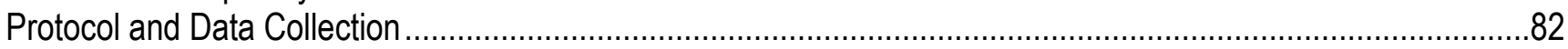

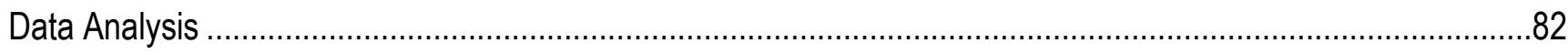

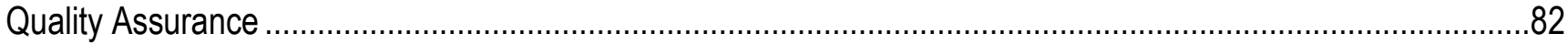

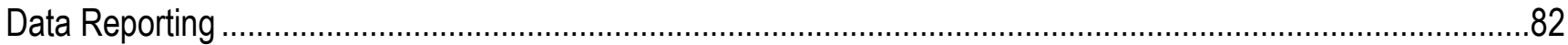

Overview of Similar Current Monitoring Activities...................................................................................83

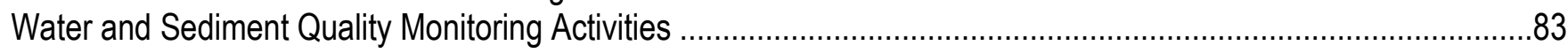

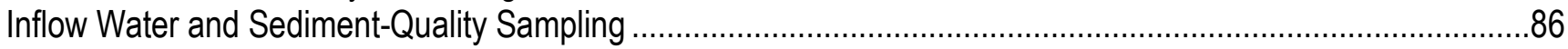

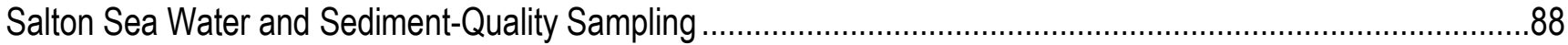

Selenium Sampling (water, sediment, and biota) …….......................................................................

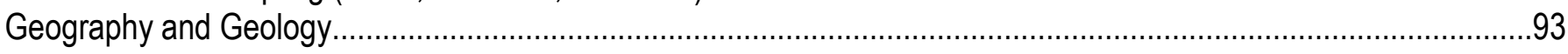




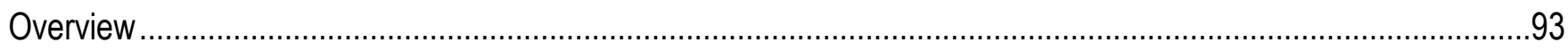

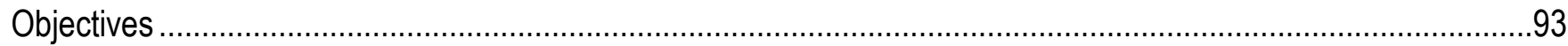

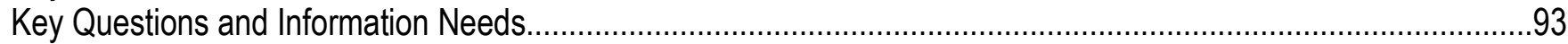

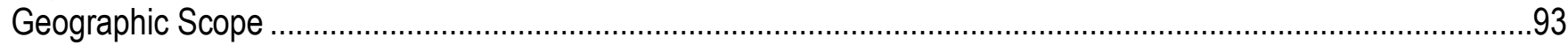

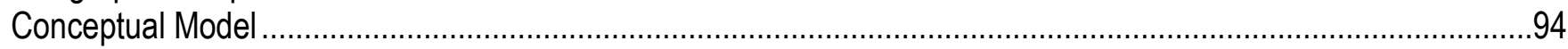

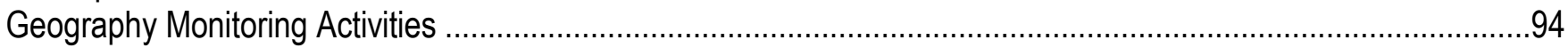

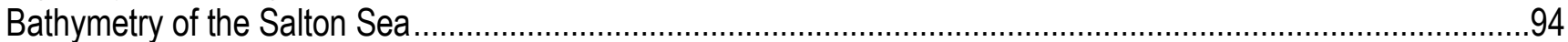

Land-Surface Elevation and Lidar .......................................................................................................

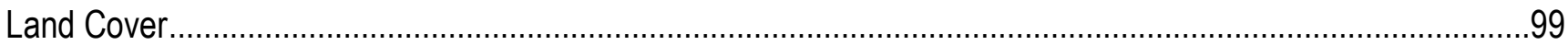

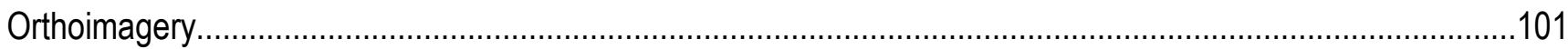

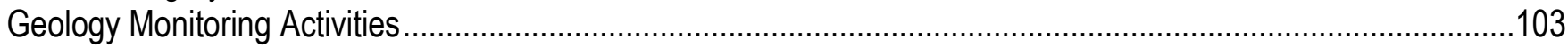

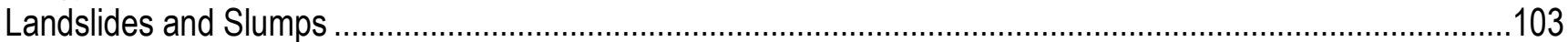

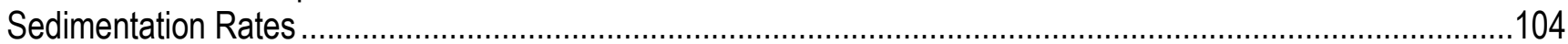

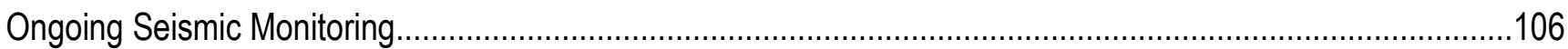

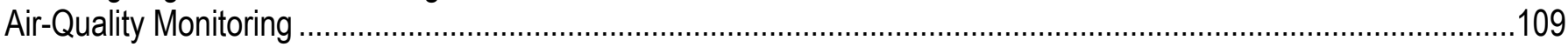

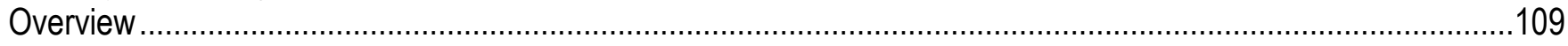

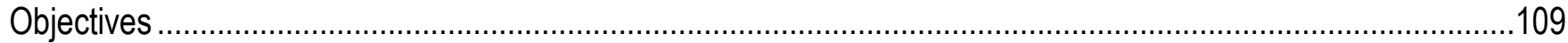

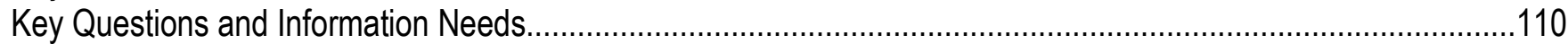

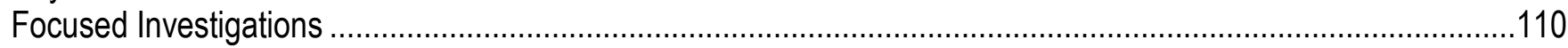

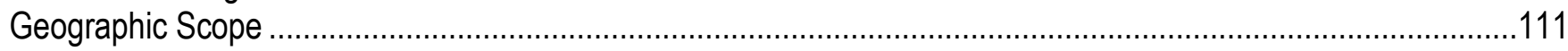

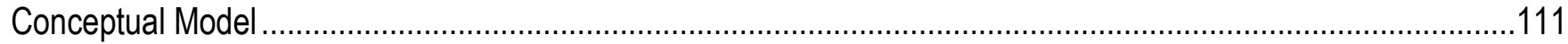

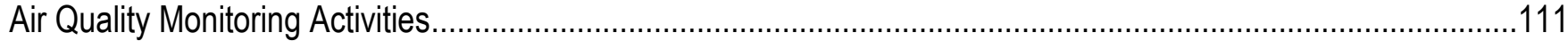

Ambient Air-Quality Monitoring .....................................................................................................112

Surface Meteorological Monitoring ..................................................................................................116

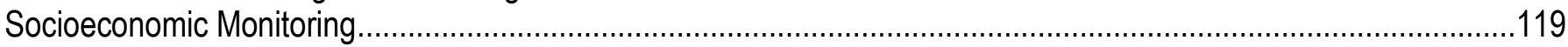

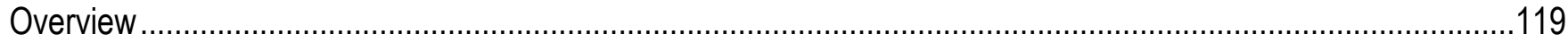

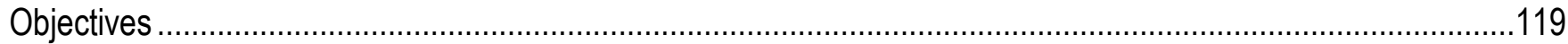

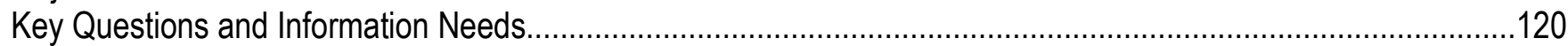

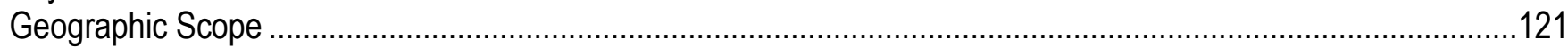

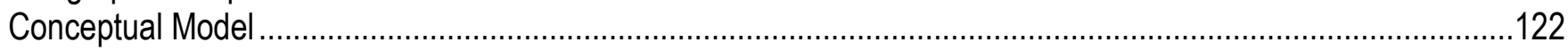

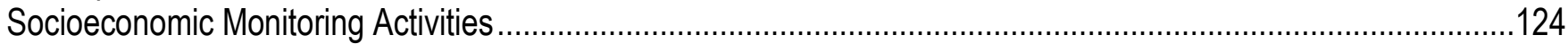

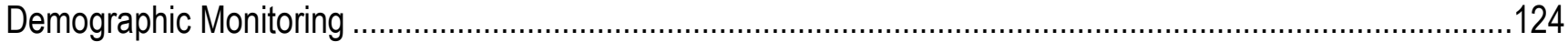

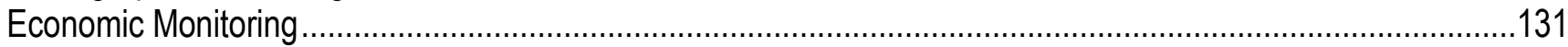

Human-Perception Monitoring .......................................................................................................132

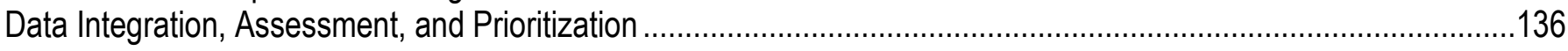

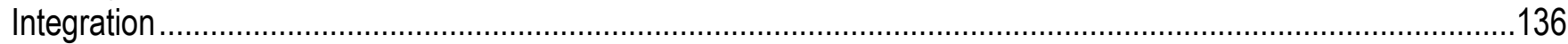

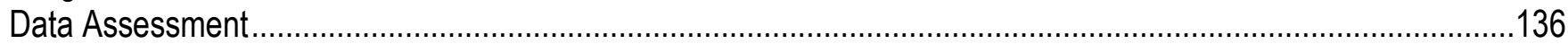

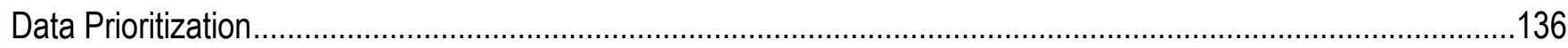

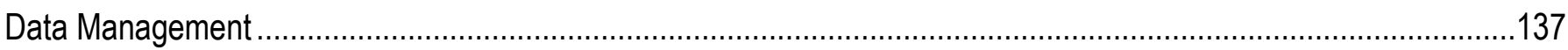

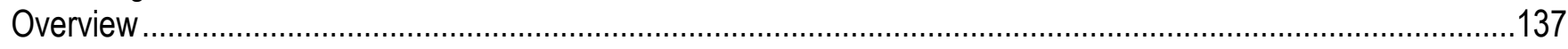

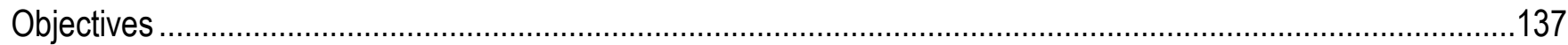

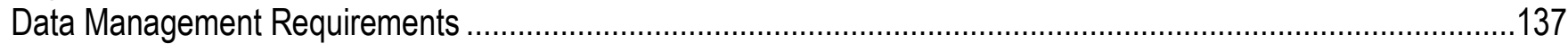

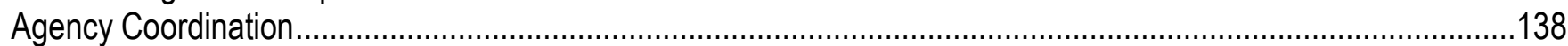

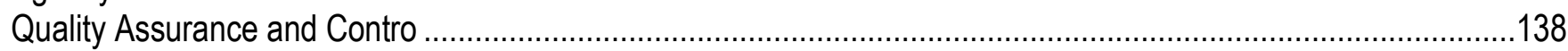

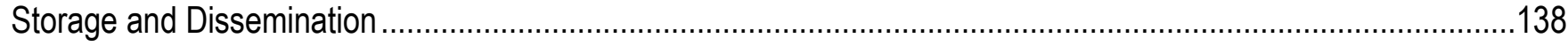

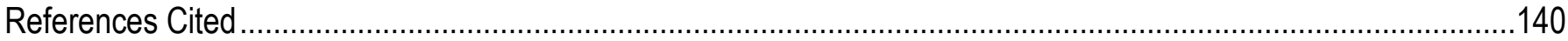

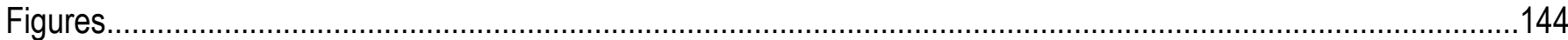

Tables 


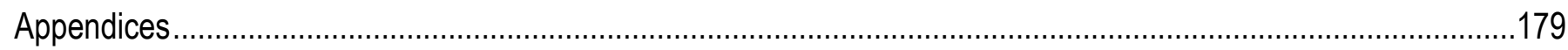

Appendix 1. Protocol for Monitoring Birds in Agricultural Areas (from ERA, 2007) ..........................................180

Appendix 2. Key Questions Identified by the Hydrology and Water Quality Focused Technical Group ...................183

Appendix 3. Conceptual Models for Selenium, Nutrient, and Sulfur Cycling at the Salton Sea.............................186

Appendix 4. SWAMP Quality Assurance Project Plan Template ......................................................................189

Appendix 5. Legacy and Current Use Pesticides Detected in the Salton Sea.................................................207

Appendix 6. Legacy and Current Use Pesticides Detected in Tributaries .........................................................208

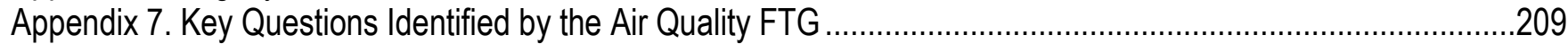

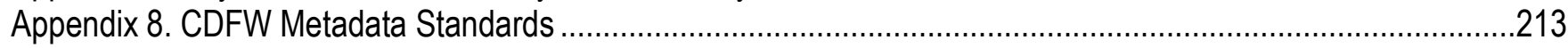

Appendix 9. Standards That Enable You to Contribute Your Data to Larger Data Sets........................................215

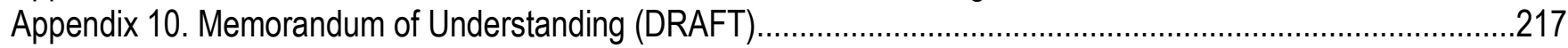

\section{Figures}

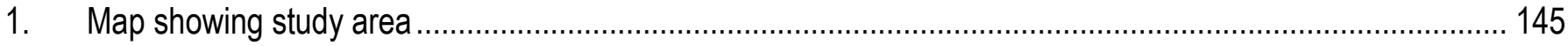

2. Diagram showing conceptual framework for the science program ........................................................... 146

3. Diagram showing potential organization of the Monitoring and Assessment Plan work group........................ 147

4. Diagram showing conceptual model for the Salton Sea Ecosystem ......................................................... 148

5. Map showing geographic strata for biological monitoring at the Salton Sea ................................................. 149

6. Diagram showing conceptual model for biological resources at the Salton Sea.............................................. 150

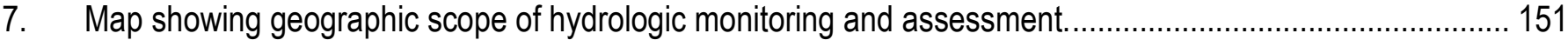

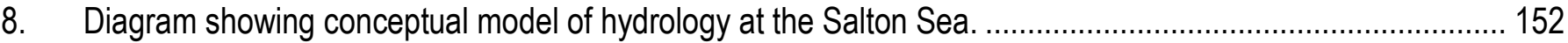

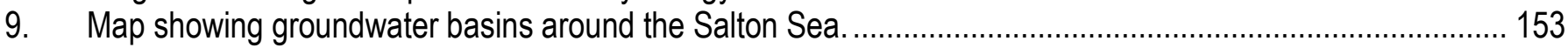

10. Map showing locations of thermistor and velocity measurement sites......................................................... 154

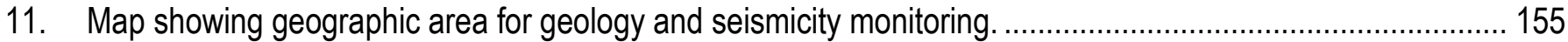

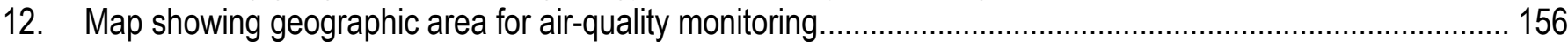

13. Diagram showing conceptual model of factors affecting air quality......................................................... 157

14. Diagram showing conceptual model of the Salton Sea socioeconomic system........................................... 158

\section{Tables}

1. Salton Sea biological resources monitoring questions and information needs........................................... 160

2. Biological resource monitoring activities and geographic strata in which they will be performed..................... 162

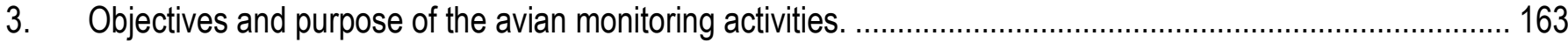

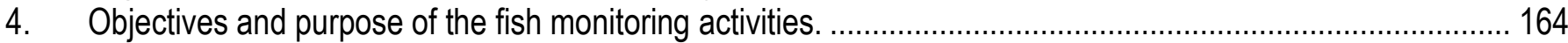

5. Objectives and purpose of the invertebrate monitoring activities. ........................................................... 165

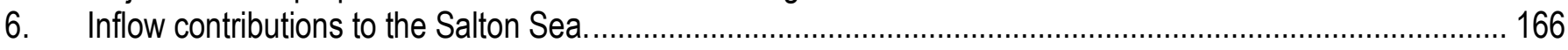

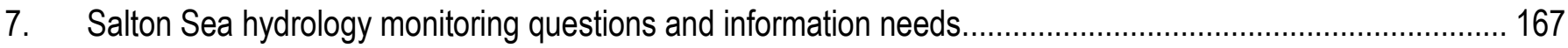

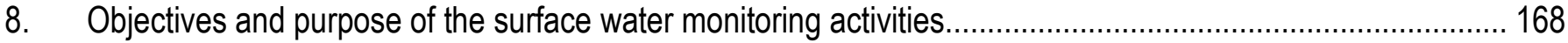

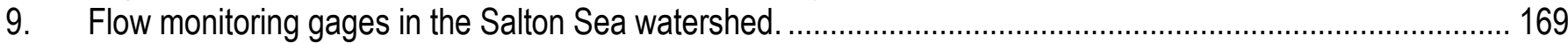

10. Objectives and purpose of shallow groundwater monitoring activities. .................................................... 170

11. Objectives and purpose of hydrodynamics (water temperature and current) monitoring activities................... 171

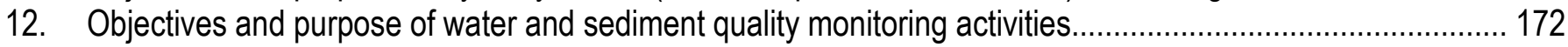

13. Location and sampling frequency for water and sediment quality parameters............................................ 173

14. Salton Sea geography and geology monitoring questions and information needs....................................... 174

15. Salton Sea air-quality monitoring questions and information needs.......................................................... 175

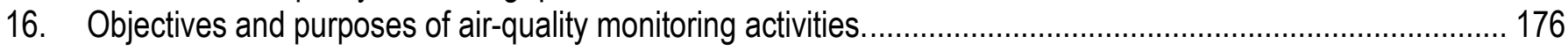

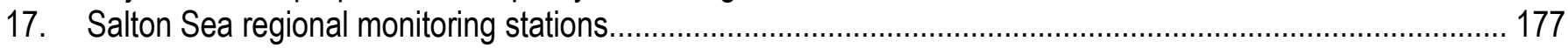

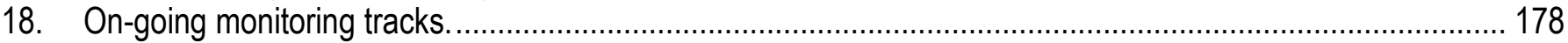




\section{Conversion Factors}

\begin{tabular}{|c|c|c|}
\hline Multiply & By & To obtain \\
\hline \multicolumn{3}{|c|}{ Length } \\
\hline inch (in.) & 2.54 & centimeter $(\mathrm{cm})$ \\
\hline inch (in.) & 25.4 & millimeter $(\mathrm{mm})$ \\
\hline foot (ft) & 0.3048 & meter $(\mathrm{m})$ \\
\hline mile (mi) & 1.609 & kilometer $(\mathrm{km})$ \\
\hline \multicolumn{3}{|c|}{ Area } \\
\hline acre & 4,047 & square meter $\left(\mathrm{m}^{2}\right)$ \\
\hline acre & 0.4047 & hectare (ha) \\
\hline square mile $\left(\mathrm{mi}^{2}\right)$ & 259.0 & hectare (ha) \\
\hline square mile $\left(\mathrm{mi}^{2}\right)$ & 2.590 & square kilometer $\left(\mathrm{km}^{2}\right)$ \\
\hline \multicolumn{3}{|c|}{ Volume } \\
\hline acre-foot (acre-ft) & 1,233 & cubic meter $\left(\mathrm{m}^{3}\right)$ \\
\hline acre-foot (acre-ft) & 0.001233 & cubic hectometer $\left(\mathrm{hm}^{3}\right)$ \\
\hline \multicolumn{3}{|c|}{ Flow rate } \\
\hline acre-foot per day (acre-ft/d) & 0.01427 & cubic meter per second $\left(\mathrm{m}^{3} / \mathrm{s}\right)$ \\
\hline acre-foot per year (acre-ft/yr) & 1,233 & cubic meter per year $\left(\mathrm{m}^{3} / \mathrm{yr}\right)$ \\
\hline cubic foot per second $\left(\mathrm{ft}^{3} / \mathrm{s}\right)$ & 0.02832 & cubic meter per second $\left(\mathrm{m}^{3} / \mathrm{s}\right)$ \\
\hline million gallons per day (Mgal/d) & 0.04381 & cubic meter per second $\left(\mathrm{m}^{3} / \mathrm{s}\right)$ \\
\hline inch per hour (in/h) & 0.0254 & meter per hour $(\mathrm{m} / \mathrm{h})$ \\
\hline inch per year (in/yr) & 25.4 & millimeter per year $(\mathrm{mm} / \mathrm{yr})$ \\
\hline mile per hour $(\mathrm{mi} / \mathrm{h})$ & 1.609 & kilometer per hour $(\mathrm{km} / \mathrm{h})$ \\
\hline \multicolumn{3}{|c|}{ Mass } \\
\hline ton, short $(2,000 \mathrm{lb})$ & 0.9072 & megagram $(\mathrm{Mg})$ \\
\hline ton per day (ton/d) & 0.9072 & metric ton per day \\
\hline ton per year (ton/yr) & 0.9072 & metric ton per year \\
\hline \multicolumn{3}{|c|}{ Pressure } \\
\hline atmosphere, standard (atm) & 101.3 & kilopascal (kPa) \\
\hline \multicolumn{3}{|c|}{ Hydraulic conductivity } \\
\hline foot per day (ft/d) & 0.3048 & meter per day $(\mathrm{m} / \mathrm{d})$ \\
\hline \multicolumn{3}{|c|}{ Application rate } \\
\hline $\begin{array}{l}\text { pounds per acre per year } \\
{[(\mathrm{lb} / \mathrm{acre}) / \mathrm{yr}]}\end{array}$ & 1.121 & $\begin{array}{l}\text { kilograms per hectare per year } \\
{[(\mathrm{kg} / \mathrm{ha}) / \mathrm{yr}]}\end{array}$ \\
\hline
\end{tabular}

Temperature in degrees Celsius $\left({ }^{\circ} \mathrm{C}\right)$ may be converted to degrees Fahrenheit $\left({ }^{\circ} \mathrm{F}\right)$ as follows: 
${ }^{\circ} \mathrm{F}=\left(1.8 \times{ }^{\circ} \mathrm{C}\right)+32$

Temperature in degrees Fahrenheit $\left({ }^{\circ} \mathrm{F}\right)$ may be converted to degrees Celsius $\left({ }^{\circ} \mathrm{C}\right)$ as follows:

${ }^{\circ} \mathrm{C}=\left({ }^{\circ} \mathrm{F}-32\right) / 1.8$ 


\begin{tabular}{|c|c|c|}
\hline Multiply & By & To obtain \\
\hline \multicolumn{3}{|c|}{ Length } \\
\hline centimeter $(\mathrm{cm})$ & 0.3937 & inch (in.) \\
\hline millimeter (mm) & 0.03937 & inch (in.) \\
\hline meter $(\mathrm{m})$ & 3.281 & foot (ft) \\
\hline kilometer (km) & 0.6214 & mile (mi) \\
\hline \multicolumn{3}{|c|}{ Area } \\
\hline square meter $\left(\mathrm{m}^{2}\right)$ & 0.0002471 & acre \\
\hline hectare (ha) & 2.471 & acre \\
\hline hectare (ha) & 0.003861 & square mile $\left(\mathrm{mi}^{2}\right)$ \\
\hline square kilometer $\left(\mathrm{km}^{2}\right)$ & 0.3861 & square mile $\left(\mathrm{mi}^{2}\right)$ \\
\hline \multicolumn{3}{|c|}{ Volume } \\
\hline liter (L) & 0.2642 & gallon (gal) \\
\hline cubic meter $\left(\mathrm{m}^{3}\right)$ & 0.0002642 & million gallons (Mgal) \\
\hline cubic meter $\left(\mathrm{m}^{3}\right)$ & 35.31 & cubic foot $\left(\mathrm{ft}^{3}\right)$ \\
\hline cubic meter $\left(\mathrm{m}^{3}\right)$ & 0.0008107 & acre-foot (acre-ft) \\
\hline cubic hectometer $\left(\mathrm{hm}^{3}\right)$ & 810.7 & acre-foot (acre-ft) \\
\hline \multicolumn{3}{|c|}{ Flow rate } \\
\hline cubic meter per second $\left(\mathrm{m}^{3} / \mathrm{s}\right)$ & 70.07 & acre-foot per day (acre-ft/d) \\
\hline cubic meter per year $\left(\mathrm{m}^{3} / \mathrm{yr}\right)$ & 0.000811 & acre-foot per year (acre-ft/yr) \\
\hline meter per second $(\mathrm{m} / \mathrm{s})$ & 3.281 & foot per second (ft/s) \\
\hline cubic meter per second $\left(\mathrm{m}^{3} / \mathrm{s}\right)$ & 35.31 & cubic foot per second $\left(\mathrm{ft}^{3} / \mathrm{s}\right)$ \\
\hline liter per second $(\mathrm{L} / \mathrm{s})$ & 15.85 & gallon per minute (gal/min) \\
\hline cubic meter per second $\left(\mathrm{m}^{3} / \mathrm{s}\right)$ & 22.83 & million gallons per day (Mgal/d) \\
\hline millimeter per year (mm/yr) & 0.03937 & inch per year (in/yr) \\
\hline kilometer per hour $(\mathrm{km} / \mathrm{h})$ & 0.6214 & mile per hour $(\mathrm{mi} / \mathrm{h})$ \\
\hline \multicolumn{3}{|c|}{ Mass } \\
\hline $\operatorname{gram}(\mathrm{g})$ & 0.03527 & ounce, avoirdupois (oz) \\
\hline kilogram (kg) & 2.205 & pound avoirdupois (lb) \\
\hline megagram (Mg) & 1.102 & ton, short $(2,000 \mathrm{lb})$ \\
\hline metric ton per day & 1.102 & ton per day (ton/d) \\
\hline metric ton per year & 1.102 & ton per year (ton/yr) \\
\hline \multicolumn{3}{|c|}{ Pressure } \\
\hline kilopascal (kPa) & 0.009869 & atmosphere, standard (atm) \\
\hline \multicolumn{3}{|c|}{ Density } \\
\hline kilogram per cubic meter $\left(\mathrm{kg} / \mathrm{m}^{3}\right)$ & 0.06242 & pound per cubic foot $\left(\mathrm{lb} / \mathrm{ft}^{3}\right)$ \\
\hline gram per cubic centimeter $\left(\mathrm{g} / \mathrm{cm}^{3}\right)$ & 62.4220 & pound per cubic foot $\left(\mathrm{lb} / \mathrm{ft}^{3}\right)$ \\
\hline
\end{tabular}




\begin{tabular}{lcl}
\hline & Hydraulic conductivity & \\
\hline meter per day $(\mathrm{m} / \mathrm{d})$ & 3.281 & foot per day $(\mathrm{ft} / \mathrm{d})$ \\
\hline & Application rate & \\
\hline $\begin{array}{l}\text { kilograms per hectare per year } \\
{[(\mathrm{kg} / \mathrm{ha}) / \mathrm{yr}]}\end{array}$ & 0.8921 & $\begin{array}{l}\text { pounds per acre per year } \\
{[(\mathrm{lb} / \mathrm{acre}) / \mathrm{yr}]}\end{array}$ \\
\hline
\end{tabular}




\section{Executive Summary}

The Salton Sea, California's largest lake, provides essential habitat for several fish and wildlife species and is an important cultural and recreational resource. It has no outlet, and dissolved salts contained in the inflows concentrate in the Salton Sea through evaporation. The salinity of the Salton Sea, which is currently nearly one and a half times the salinity of ocean water, has been increasing as a result of evaporative processes and low freshwater inputs. Further reductions in inflows from water conservation, recycling, and transfers will lower the level of the Salton Sea and accelerate the rate of salinity increases, reduce the suitability of fish and wildlife habitat, and affect air quality by exposing lakebed playa that could generate dust.

Legislation enacted in 2003 to implement the Quantification Settlement Agreement (QSA) stated the Legislature's intent for the State of California to undertake the restoration of the Salton Sea ecosystem. As required by the legislation, the California Resources Agency (now California Natural Resources Agency) produced the Salton Sea Ecosystem Restoration Study and final Programmatic Environmental Impact Report (PEIR; California Resources Agency, 2007) with the stated purpose to "develop a preferred alternative by exploring alternative ways to restore important ecological functions of the Salton Sea that have existed for about 100 years." A decision regarding a preferred alternative currently resides with the California State Legislature (Legislature), which has yet to take action.

As part of efforts to identify an ecosystem restoration program for the Salton Sea, and in anticipation of direction from the Legislature, the California Department of Water Resources (DWR), California Department of Fish and Wildlife (CDFW), U.S. Bureau of Reclamation (Reclamation), and U.S. Geological Survey (USGS) established a team to develop a monitoring and assessment plan (MAP). This plan is the product of that effort.

The goal of the MAP is to provide a guide for data collection, analysis, management, and reporting to inform management actions for the Salton Sea ecosystem. Monitoring activities are directed at species and habitats that could be affected by or drive future restoration activities. The MAP is not intended to be a prescriptive document. Rather, it is envisioned to be a flexible, program-level guide that articulates highlevel goals and objectives, and establishes broad sideboards within which future project-level investigations and studies will be evaluated and authorized. As such, the MAP, by design, does not, for example, include detailed protocols describing how investigations will be implemented. It is anticipated that detailed study proposals will be prepared as part of an implementation plan that will include such things as specific sampling objectives, sampling schemes, and statistical and spatial limits.

Monitoring of the Salton Sea ecosystem is critical for informed decision making and the success of restoration efforts. Information derived from monitoring activities will be used to guide the initial designs and management of restoration actions. Monitoring will also help ensure the success of management actions by identifying actions that are not having the desired effect so that they can be adjusted. The MAP is intended to foster the following specific objectives:

- Establish baseline conditions for the Salton Sea ecosystem.

- Establish metrics against which data gathered during long-term monitoring can be compared.

- Identify and prioritize filling of existing data gaps.

- Store, manage, and make monitoring data publicly available in a timely manner.

The scope of the MAP is intentionally broad to capture the potential range of processes and conditions influencing the Salton Sea ecosystem and its associated resources. By adopting a broad scope for the MAP, investigators will be able to identify linkages and interactions among resources and integrate 
monitoring actions needed to make full use of the collected data. The comprehensive nature of the plan also reflects the current uncertainty regarding the ultimate intent of restoration and the desire to collect an array of information that will not preclude informing specific restoration actions or alternatives.

The MAP will be subject to regularly scheduled reviews, and revised as needed as monitoring needs, technologies, and assessment methods evolve. Further, the initial monitoring protocols identified in the MAP could change over time as new and improved techniques and assessment methods are identified. Because many of the previously mentioned objectives have data as a component of the objective, data management through the entire life cycle of monitoring and assessment program is included within the scope of the MAP. Data management will be the responsibility of individual investigators and coordinated through the CDFW, as described in this plan.

The MAP is envisioned to be one of several components of an integrated science program that would coordinate and oversee (including peer review) data collection, data analysis (including a retrospective analysis), and an adaptive management strategy to support future restoration actions at the Salton Sea. This framework envisions a science integration and coordination entity that would answer questions relevant to policy and decision making by using the best available science and an adaptive management approach based on integrated assessment of data developed through the MAP, results of focused investigations, and a retrospective analysis of prior data and existing literature.

Identifying and prioritizing monitoring activities that would be implemented under this MAP will be the subject of a detailed implementation plan. The implementation plan will establish the elements of the MAP that should be performed and the scope of those activities. Monitoring activities to be performed will be identified on the basis of information needed for future environmental documentation, information needed to help resolve uncertainty, and information needed to provide a basis for comparison. Funding and logistics for the actual field work will be considered in development of the implementation plan.

This MAP is organized by separate, potentially stand-alone modules, describing monitoring of biological, hydrologic, geographic and geologic, air quality and climate, and socioeconomic resources, as well as data management activities. Each module includes a general overview, the monitoring objectives, key questions and information needs, the geographic scope, conceptual models, and recommended monitoring activities. Each section describing recommended monitoring activities includes a description of the purpose and justification for the monitoring activity, location, period and frequency of monitoring, protocol for data collection, a description of the data to be collected and the anticipated use of the data, recommended quality-assurance measures, data reporting guidelines, and an overview of similar current monitoring activities. Opportunities for coordination of activities are generally identified in the section on period and frequency of monitoring. References cited are part of each module, and additional supporting documentation is included at the end of the report in the appendices.

This MAP was developed with input from a wide variety of subject matter experts including scientists, managers, regulators, and staff representing a range of state, federal, academic, tribal, local, industry, and non-governmental organizations. The overall intent was to be inclusive of the scientific community to assure that monitoring needs could be reasonably anticipated. A slightly modified version of the data-quality objective (DQO) process (US Environmental Protection Agency, 2006) was used to help guide development of the MAP. This process included stating the problem, identifying the goal of the study, identifying the information inputs, defining the boundaries, developing the analytical approach, and developing the plan for obtaining the data. Focused technical groups (FTGs) of technical experts for each resource (biology, hydrology, geography and geology, air quality and climate, and socioeconomics) were tasked with identifying the purpose and objectives for monitoring of their specific resources and developing key questions (see appendices) that could be answered through monitoring, developing conceptual models, identifying specific monitoring activities, and identifying needed coordination and integration with other resource areas. 
This monitoring plan was developed within the context of the final PEIR prepared by the California Natural Resources Agency (California Resources Agency, 2007) and the future direction of ecosystem restoration defined by that process. Several assumptions related to decisions made through the development of the PEIR were used to further focus the initial monitoring plan. These relate primarily to the expected direction and configuration of ecosystem restoration, given what is currently known about the system, and factors that likely will influence the design and management of restoration features. These assumptions could change when the California Legislature provides direction on the restoration and identifies an implementing agency. These constraints and assumptions were grouped under one of the following categories: programmatic, environmental, engineering and physical, hydrological, and legal.

All monitoring activities recommended in this MAP are intended to help answer key questions anticipated to be asked by managers responsible for restoration of the Salton Sea ecosystem. These key questions were identified through brainstorming and a subsequent grouping process by FTG members. Not all questions identified by the FTGs could reasonably be expected to be answered by monitoring alone. Answers to some questions will need to be addressed by focused investigations or by specific studies and research. Focused investigations are not part of this MAP; however, some potential investigations and studies were identified by the FTGs and are included in a listing of key questions for each resource in the appendices.

Simplified conceptual models were developed for each major resource area. These models serve as initial starting points upon which to build more robust models and a clearer understanding of the overall functioning of the Salton Sea ecosystem. The conceptual models guide both long-term monitoring and focused investigations toward goals and objectives identified for restoration.

The development of this document was born out of a desire to establish common sets of processes and procedures to which all scientists can adhere as well as a framework for future science activities. This document further aspires to promote the integration of ideas, activities, and sampling strategies and locations to the greatest practical extent whenever possible. Success or failure in restoration of the Salton Sea ecosystem rests heavily on the degree to which science, through monitoring and focused investigations, can provide resource managers with skillful guidance for adaptive management. This guidance rests squarely on implementation of a rigorous framework for science, integration of all program activities, and incisive analysis.

Considerable attention was given to monitoring, design, and strategy as implementation of the MAP was contemplated, including retrospective analysis, quality assurance (QA), data assessment, peer review, and adaptive management. Retrospective data are data collected in the past that provide a foundation for the identification of data gaps, questions, and unknowns, which, in turn, will be used to identify specific data that will be collected under this MAP. Quality assurance is a critical element of all monitoring programs. Inclusion of a QA element helps ensure that the type, amount, and quality of data collected are adequate to meet study objectives. It is anticipated that focused investigations will be used in conjunction with data collected through the monitoring program to answer key questions and to assist with efforts to adaptively manage restoration activities. Data, analyses, and publications developed from this plan will be organized, stored, and made publicly accessible through a common distributed data management system (that is, the CDFW's Biogeographic Information and Observation System, or BIOS). Data collected and stored within the data management system will be publicly available for the application of statistical and other analytical techniques. This step helps to simplify the collected data, test for change and differences, develop and test hypotheses, and evaluate uncertainty. Data assessment is used to foster the integration, consolidation, and review of data, updating of the conceptual models, answering of key questions, reporting, and providing management recommendations within an adaptive management framework. Peer review is a critical step to help ensure that data collection and analyses are conducted in a manner that provides defensible scientific conclusions or findings. Adaptive management entails feedback between management practices and monitoring of responses in the ecosystem in order to measure the 
success of management actions and to fine-tune future actions accordingly. In practice, this means that science is used to design the actions, and then monitoring results are provided to managers overseeing restoration so that adjustments can be made, if needed. This feedback works best if scientists are engaged in the design of restoration actions as experiments whose outcomes can be predicted and then measured.

In cooperation with technical and management staff, and with input from stakeholder groups, the assessment and analysis of data are anticipated to lead to periodic updates of this entire MAP. Updates could be focused on specific resource areas or be applied to the entire plan. The plan is envisioned to be a living document and will need to remain flexible in order to respond effectively to unanticipated events. 


\title{
Salton Sea Ecosystem Monitoring and Assessment Plan
}

\author{
By H.L. Case III', Jerry Boles², Arturo Delgado³, Thang Nguyen², Doug Osugi2, Douglas A. Barnum¹, Drew
} Decker $^{1}$, Steven Steinberg ${ }^{4}$, Sheila Steinberg ${ }^{5}$, Charles Keene ${ }^{2}$, Kristina White ${ }^{3}$, Tom Lupo ${ }^{3}$, Sheldon Gen ${ }^{6}$, and Ken A. Baerenklau

\section{Introduction}

The Salton Sea, California's largest lake, provides essential habitat for several fish and wildlife species and is an important cultural and recreational resource. It has no outlet, and dissolved salts contained in the inflows concentrate in the Salton Sea through evaporation. The salinity of the Salton Sea, which is currently nearly 1.5 times the salinity of ocean water, has been increasing as a result of evaporative processes and low freshwater inputs. Further reductions in inflows due to water conservation, recycling, and transfers will lower the level of the Salton Sea and accelerate the rate of salinity increases, reduce the suitability of fish and wildlife habitat, and affect air quality by exposing lakebed playa that could generate dust.

Legislation enacted in 2003 to implement the Quantification Settlement Agreement (QSA) stated the Legislature's intent for the State of California to undertake the restoration of the Salton Sea ecosystem. As required by the legislation, the Salton Sea Ecosystem Restoration Study and Programmatic Environmental Impact Report (PEIR) was produced by the California Resources Agency (2007, name has been changed to California Natural Resources Agency). Its stated purpose was to "develop a preferred alternative by exploring alternative ways to restore important ecological functions of the Salton Sea that have existed for about 100 years." A decision regarding the implementation of a preferred alternative currently resides with the California State Legislature (Legislature), which has yet to take action.

As part of the efforts to identify an ecosystem restoration program for the Salton Sea, and in anticipation of direction from the Legislature, the California Department of Water Resources (DWR), California Department of Fish and Wildlife (CDFW), U.S. Bureau of Reclamation (Reclamation), and U.S. Geological Survey (USGS) established a team to develop a Monitoring and Assessment Plan (MAP). This plan is the product of that effort.

\section{Goal and Objectives}

The goal of the MAP is to provide a guide for data collection, analysis, management, and reporting to inform management actions for the Salton Sea ecosystem. Monitoring activities are directed at species and habitats that could be affected by or drive future restoration activities. The MAP is not intended to be a prescriptive document. Rather, it is envisioned to be a flexible, program-level guide that articulates high-level goals and objectives and establishes broad sideboards within which future projectlevel investigations and studies will be evaluated and authorized. As such, the MAP does not, for example, include detailed protocols describing how investigations will be implemented by design. It is anticipated that detailed study proposals will be prepared as part of an Implementation Plan that will include such things as specific sampling objectives, sampling schemes, and statistical and spatial limits.

${ }^{1}$ U.S. Geological Survey; ${ }^{2}$ California Department of Water Resources; ${ }^{3}$ California Department of Fish and Wildlife; ${ }^{4}$ Southern California Coastal Water Research Project; ${ }^{5}$ Brandman University; ${ }^{6}$ San Francisco State University; ${ }^{7}$ University of California, Riverside 
Monitoring of the Salton Sea ecosystem is critical for informed decision making and to the success of restoration efforts. Information derived from monitoring activities will be used to guide the initial designs and management of restoration actions. Monitoring will also help ensure the success of management actions by identifying actions that are not having the desired effect so that the action(s) can be adjusted.

The MAP is intended to foster the following specific objectives:

- Establish baseline conditions for the Salton Sea ecosystem.

- Establish metrics against which data gathered during long-term monitoring can be compared.

- Identify and prioritize filling of existing data gaps.

- Store, manage, and make monitoring data publicly available in a timely manner.

\section{Scope}

The scope of the MAP is intentionally broad to capture the range of processes and conditions that potentially influence the Salton Sea ecosystem and its associated resources. By adopting a broad scope for the MAP, investigators can identify linkages and interactions among resources and the integrate monitoring to make full use of the resulting dataset. The comprehensive nature of the plan also reflects the current uncertainty regarding the direction of restoration and the desire to collect an array of information that does not preclude specific restoration actions or alternatives. Once direction is provided by the California Legislature regarding restoration activities, it is anticipated that monitoring activities under this plan would be refined and focused to meet the specific objectives of the selected restoration alternative. In lieu of further guidance from the legislature, the MAP is intended to guide managers as they implement the monitoring and assessment program to identify and select specific monitoring actions most appropriate for developing a technical foundation that facilitates future restoration activities.

The MAP will be reviewed regularly, and revised, as needed, as monitoring needs, technologies, and assessment methods evolve. Further, the initial monitoring protocols identified in the MAP can change over time as new and improved techniques and assessment methods are identified. Because many of the previously stated objectives include data as a component, data management during the entire monitoring and assessment program is within the scope of the MAP. Data management will be the responsibility of individual investigators and coordinated through the CDFW as described in this plan.

\section{Study Area}

The assessment area for the MAP spans the Salton Sea ecosystem, which is defined in Section 2931 of the Fish and Game Code as including, but not limited to, "the Salton Sea, the agricultural lands surrounding that lake, and the tributaries and drains within the Imperial and Coachella valleys that deliver water to the Salton Sea." The areas included in this MAP vary with the resources or factors being monitored, however. Most monitoring will take place in the area shown in figure 1. Specific areas are identified for each monitoring element depending on the processes influencing each resource or factor of interest. For example, the watershed boundary defines the area of hydrologic monitoring. The air-quality study area extends beyond the watershed boundary because the processes that govern wind erosion and deposition are not limited to the watershed boundary. 


\section{Implementation}

The MAP is envisioned to be one of several components of an integrated science program that coordinates and oversees (including peer review) data collection, data analysis (including a retrospective analysis), and an adaptive-management strategy to inform and guide future restoration actions at the Salton Sea. A conceptual framework for the science program is presented in figure 2. This framework envisions a science integration/coordination entity that could answer questions relevant to policy and decision making by using the best available science and an adaptive management approach based on integrated assessment of data developed through the MAP, the results of focused investigations, and a retrospective analysis of existing data and literature.

The specific structure of the monitoring and assessment component of the Science Program is uncertain; however, an example of how this component could be structured is illustrated in figure 3. Under this structure, a monitoring and assessment work team would be assembled and led by a MAP Science Coordinator, who reports to and takes direction from the Science Integration and Coordination entity. The MAP work team would be composed of leaders of focused technical groups of subject matter experts representing relevant technical disciplines that are responsible for identifying, coordinating, integrating, and prioritizing monitoring activities within their respective disciplines, given the budgetary and other technical constraints identified by the Science Integration and Coordination entity. These focused technical groups have been instrumental in providing input during preparation of the MAP. The MAP Science Coordinator and work team could help guide monitoring efforts, identify data gaps, avoid potential duplication of efforts, and solicit efforts, when needed, to fill high-priority gaps. The Science Integration and Coordination entity, with input and support from the MAP work team, would be responsible for assuring that long-term and short-term high priority monitoring needs are met.

Identifying and prioritizing monitoring activities that would be implemented under this MAP will be the subject of a detailed implementation plan. The implementation plan will establish which elements of the MAP are priorities to be performed and the scope of those activities. Monitoring activities to be performed will be identified on the basis of information needed for future environmental documentation, information needed to help resolve uncertainty, and information needed to provide a basis for comparison. Funding and logistics for the field work needed to collect the data will be assessed during the development of the implementation plan. The prioritization of monitoring activities will account for the rapidly changing conditions at the Salton Sea.

The implementation plan will describe the specific protocols, procedures, and analysis techniques to be used during the selected monitoring activities. The implementation plan will also describe the process for assessment and management of data collected during MAP activities. Time is of the essence for many of the initial restoration actions, and some actions (for example, habitat creation) could need to proceed without the benefit of additional monitoring data.

\section{Background}

The Salton Sea lies in a closed desert basin in Riverside and Imperial counties in southern California (see figure 1). The basin, which extends from Banning, California, on the north, to near the international border with Mexico on the south, is composed of sediments deposited from adjacent mountains and from sediments deposited by the Colorado River. These sediments reach a maximum thickness of 21,000 feet near the Mexican border (Barker, 1995).

The Salton Sea has no natural outlet and fluctuates in size as inflows vary. Although natural lakes have existed in this basin historically, the current body of water was formed in 1905 when the Colorado River flowed through a levee break into the basin for about 18 months. Since 1905, the lake 
has been sustained by inflows primarily composed of agricultural runoff. At a water-surface elevation of approximately 230 feet below mean sea level (msl), the surface area of the Salton Sea is about 365 square miles; maximum depth of the lake is about 50 feet. Salt concentrations in the Salton Sea were about 50,000 milligrams per liter (mg/L) as of 2009 (Chris Holdren, written commun., 2009).

\section{Salton Sea Restoration}

\section{Legislation}

Historically, California used more than its annual interstate apportionment of 4.4 million acre feet from the Colorado River; as the States of Arizona and Nevada began to approach their full apportionments, California was faced with reducing its reliance on the Colorado River. After prolonged negotiations between the Federal Government and the water districts that hold rights to Colorado River water within California, a series of agreements, collectively known as the Quantification Settlement Agreement (QSA), were made in October 2003 among the Federal Government, State of California, Imperial Irrigation District (IID), Metropolitan Water District of Southern California, Coachella Valley Water District (CVWD), and San Diego County Water Authority. One element of the QSA facilitates the transfer of an increment of water used for agricultural irrigation out of the Salton Sea basin. This action, in combination with other factors, such as water recycling in Mexico, is anticipated to contribute to a reduction in Salton Sea inflow and an acceleration of salinity increase and water-level decline.

Among the several pieces of legislation supporting the QSA, California Senate Bill 187, signed by Governor Schwarzenegger on September 27, 2008, requires that bond funds deposited into the Salton Sea Restoration Fund from the Safe Drinking Water, Water Quality and Supply, Flood Control, River and Coastal Protection Bond Act of 2006 (Proposition 84) "be expended for a Salton Sea restoration project that is consistent with a preferred alternative that provides the maximum feasible attainment of the specified environmental objectives." Expenditure of these funds depends on appropriation by the Legislature in the annual Budget Act. The bill limits "activities and expenditures authorized by the bill to funding those activities identified in a specific preferred alternative report and funding plan for completion in the first 5 years of implementation." Activities identified for completion in the first 5 years (Period I) include a demonstration project, early-start habitat, and additional geotechnical, biological, inflow and sediment, water, and air-quality investigations.

In Federal legislation, the 2007 Water Resources and Development Act authorizes the Secretary of the Army to review the plan approved by the State, entitled the "Salton Sea Ecosystem Restoration Program Preferred Alternative Report and Funding Plan," to determine whether the pilot projects described in the plan are feasible. The act authorizes the Secretary to enter into an agreement with the State to carry out pilot projects for improvement of the environment in the area of the Salton Sea in consultation with the Salton Sea Authority and the USGS Salton Sea Science Office.

\section{Programmatic Environmental Impact Report (PEIR)}

Legislation enacted in 2003 to implement the QSA stated the Legislature's intent for the State of California to undertake the restoration of the Salton Sea ecosystem. As required by the legislation, the California Natural Resources Agency released the Salton Sea Ecosystem Restoration Draft PEIR (California Resources Agency, 2006). The purpose of the PEIR was to "develop a preferred alternative by exploring alternative ways to restore important ecological functions of the Salton Sea that have existed for about 100 years." The draft PEIR described eight alternatives and compared the alternatives

to both existing conditions and the no action alternative. The draft PEIR provided the foundation for the Salton Sea Ecosystem Restoration Program's Final Programmatic Environmental Impact Report 
(California Resources Agency, 2007) and Certification. The final PEIR includes a description of the Preferred Alternative, all of the comments received during the 90-day review period, and responses to those comments. In May 2007, the Secretary for the Resources Agency provided to the Legislature, in accordance with Fish and Game Code 2081.7, the Salton Sea Ecosystem Restoration Program Preferred Alternative Report and Funding Plan, and Ecosystem Restoration Study.

\section{Document Organization and Intent}

This MAP is organized by separate, potentially stand-alone modules, which describe approaches to monitoring of biological, hydrologic, geographic and geologic, air-quality and climatic, and socioeconomic resources, as well as data-management needs and activities. Each module includes a general overview, the monitoring objectives, key questions and information needs, the geographic scope, conceptual models, and recommended monitoring activities. Each section describing proposed monitoring activities includes a description of the purpose and justification for the monitoring activity, location, period and frequency of monitoring, protocols for data collection, a description of the data to be collected and the anticipated use of the data, recommended quality-assurance measures, data reporting guidelines, and an overview of similar current monitoring activities. Opportunities for coordination of activities are generally identified in the section on period and frequency of monitoring. References are cited as needed for each module, and additional supporting documentation is included at the end of the report in the appendixes.

\section{Plan Development}

This MAP was developed with input from a wide variety of subject matter experts including scientists, managers, regulators, and staff representing a range of state, federal, academic, tribal, local, industry, and non-governmental organizations. The overall intent was to involve the scientific community to assure that monitoring needs could be reasonably anticipated. A slightly modified version of the data quality objective (DQO) process (US Environmental Protection Agency, 2006) was used to help guide development of the MAP. This process included stating the problem, identifying the goal of the study, identifying the information inputs, defining the boundaries, developing the analytical approach, and developing the plan for obtaining the data.

\section{Focused Technical Groups}

Focused Technical Groups (FTGs) for each resource area (biology, hydrology, geography and geology, air quality and climate, socioeconomics, and data management) were formed beginning in November 2007. Each FTG held meetings with invited technical experts. The FTGs were tasked with identifying the purpose and objectives for monitoring of their specific resources and developing key questions (see appendixes) that could be answered through monitoring, developing conceptual models for each resource, identifying specific monitoring activities, and identifying needed coordination and integration with other disciplines.

\section{Constraints and Assumptions}

This monitoring plan was developed within the context of the final PEIR prepared by the Resources Agency (California Resources Agency, 2007) and the future direction of ecosystem restoration defined by that process. Because of the uncertainty regarding the direction that the Legislature will take with restoration of the Salton Sea, a primary consideration in the development of this monitoring plan was that information collected as part of the initial monitoring program must be 
useful for informing and guiding management actions that could be implemented with or without a restoration program. Accordingly, the initial monitoring plan emphasizes monitoring activities at the Salton Sea itself.

Several assumptions related to decisions made through the development of the PEIR were used to further focus the initial monitoring plan. These relate primarily to the expected direction and configuration of ecosystem restoration, given what is currently known about the system and factors that likely will influence the design and management of restoration features. These assumptions could change when the California Legislature provides direction on the restoration and identifies an implementing agency. The following constraints and assumptions about the potential restoration influenced the development of the monitoring plan.

Program Constraints or Assumptions

- Information collected as part of the monitoring plan will be used in an adaptive management framework to inform and guide management actions that could be implemented with or without a restoration program.

\section{Environmental Constraints or Assumptions}

- Replacement of Salton Sea habitat values and functions will be attained by the creation of habitat within the existing footprint of the lake, and not by maintaining the Salton Sea in its entirety.

- Created habitats will not replicate all habitat types of the current Salton Sea ecosystem, but will be designed to replace functional ecologically equivalent values.

- Model projections indicate that salinity levels are expected to exceed 60,000 milligrams per liter $(\mathrm{mg} / \mathrm{L})^{2}$ by 2018 , thereby resulting in the loss of the tilapia population that supports fish-eating birds.

- Additional fish or invertebrate species could be introduced to created habitats to increase diversity and improve ecosystem function.

Engineering and Physical Constraints or Assumptions

- Any future habitat creation will take place below elevation -230 feet msl (primarily on exposed lakebed).

Hydrological Constraints or Assumptions

- Future sources of inflow water needed to implement management actions will remain unchanged from the current sources.

Legal Constraints or Assumptions

- Any management actions will need to comply with applicable laws.

${ }^{2}$ While milligrams per liter $(\mathrm{mg} / \mathrm{L})$ is the standard unit by which salinity at the Salton Sea has been reported, future monitoring will measure salinity in terms of electrical conductance normalized to $25^{\circ}$ Celsius as "specific conductance." 


\section{Key Questions and Monitoring Activities}

All monitoring activities recommended in this MAP are directed to help answer key questions anticipated to be asked by managers responsible for restoration of the Salton Sea ecosystem. These key questions were identified through brainstorming and a subsequent grouping process by FTG members. Not all questions identified by the FTGs could reasonably be expected to be answered by monitoring alone. Answers to some questions will need to be addressed by focused investigations or by specific studies and research. Focused investigations are not part of this MAP; however, some potential investigations and studies were identified by the FTGs and are included in a listing of key questions for each resource in the appendices.

\section{Conceptual Models}

Simplified conceptual models were developed for each major topic of investigation. These simplified models will serve as initial starting points upon which to build a more robust model and understanding of the overall functioning of the Salton Sea ecosystem and its various contributing elements. An example of a simple conceptual model for the Salton Sea ecosystem was developed by the Salton Sea Science Subcommittee (2000) and is shown in figure 4. The model "emphasizes the interactions between the terrestrial and quasi-marine elements, as well as interactions between the biota and their environment. The value of this simple conceptual model is that it reinforces the interactions of major ecosystem elements." In design of the MAP, conceptual models for each resource area were developed to guide the construction of quantitative models by identifying ecosystem processes and functions thought to be important to the resource. The conceptual models guide both long-term monitoring and focused investigations toward goals and objectives identified for restoration.

\section{Monitoring Activities}

By using the key questions and the conceptual models developed for each MAP element to clarify processes thought to be important for guiding management of the various resource areas, members of each FTG categorized monitoring activities into specific sub-disciplines to help ensure that an appropriate level of detail was developed and included for all monitoring. For example, the Hydrology FTG organized its recommended monitoring activities into surface-water, groundwater, hydrodynamics, and water and sediment-quality categories. Within each sub-discipline, specific activities (such as measurement of surface-water flows or elevations) were identified. For each monitoring activity, the purpose and justification, location, period and frequency, protocol and data collection (description of data to be collected and how it is collected), data analysis (the expected use of data in subsequent analyses), quality-assurance expectations, and an overview of similar current monitoring activities are presented.

For all monitoring activities, a detailed monitoring protocol that explains how data are to be collected, managed, analyzed, and reported will be developed prior to implementation. Protocols will be developed by subject-matter experts who may also perform the monitoring activity. The spatial and temporal extent of the monitoring activity will be scaled to meet the specific objectives and level of resolution desired (statistical power). As the detailed protocols are developed, they will be peer reviewed and incorporated into the MAP, which will be updated periodically to include the development or refinement of monitoring protocols. 


\section{Monitoring Design and Strategy}

\section{Retrospective Analysis}

Retrospective data were collected in the past and can help identify data gaps, questions, and unknowns, which in turn, will be used to identify specific data to be collected under this MAP. A substantial amount of retrospective data has been collected and is available through a variety of sources. These sources include, but are not limited to, federal agencies, state agencies; Torres Martinez and Agua Caliente Native American Tribes, Riverside and Imperial Counties, University of Redlands, Imperial Irrigation District (IID), Metropolitan Water District of Southern California, and non-governmental organizations. Existing relevant information will be gathered and assessed to determine if the data conform to predetermined quality standards (including metadata requirements) and will be useful for meeting the objectives of the MAP. Collecting, compiling, and evaluating retrospective data will help identify where sufficient data exist to make decisions to proceed with specific restoration actions.

\section{Quality Assurance}

Quality assurance (QA) is a critical element of all monitoring programs. Inclusion of a QA element helps ensure that the type, amount, and quality of data collected are adequate to meet study objectives. The Strategic Science Plan for the Department of the Interior's Salton Sea Restoration Project (Salton Sea Science Subcommittee, 2000) notes "data collected in the monitoring program must be quality assured and quality controlled at all points in the process. Sampling, sample labeling, preservation, storage, transport, analysis, and data entry and compilation must all follow established and accepted guidelines. Any automated analysis must follow guidelines of calibration and standardization." State of California requirements for data management also include specific quality-control requirements. State and federal agencies could have specific quality-control and quality-assurance guidelines that will need to be met. A QA element is included in each element of the MAP and is referenced in each module. QA plans will be modified and updated as necessary and as consistent with the "living document" philosophy of this MAP.

\section{Peer Review}

Peer review is a critical step to help ensure that data collection and analyses are performed in a manner that provides defensible scientific conclusions or findings. Peer review is defined in the USGS Manual (section 502.3) as "scrutiny of work or ideas by one or more others (peers) who are sufficiently well qualified, who are without conflict of interest, and who are not associated with the work being performed. A peer is defined as one who is of equal standing with another; in science, the implication is that education and/or experience qualify one to comment on the work of others in a particular field of expertise. These persons may be internal or external to the organizational entity in which the review is conducted."

This MAP and data collected through this plan will be subject to peer review. Data analyses and focused investigations also need to be submitted through peer reviews. Data collected through this plan also may be subjected to management reviews (assessments of funding, time frames, personnel requirements, and so on; editorial reviews (for expression, clarity, organization, and so on); and policy reviews (compliance with agency policy and practice) by funding and partner agencies. Reviews are anticipated to be commensurate with the complexity of the proposed activity and consistent with processes identified in the quality-assurance plans. Data collected with public funds are anticipated to be publically available upon review and evaluation. 


\section{Focused Investigations}

As previously described, many of the fundamental information gaps that exist at the Salton Sea cannot be addressed by monitoring alone, and focused investigation and integrated assessment of existing data will be necessary. Determining ecosystem cause-and-effect relationships, for example, will require tests of hypotheses generated by conceptual and quantitative models. This information is critical to determine the functional linkages among ecosystem components and to refine and validate conceptual models. Other information gaps will require one-time surveys in order to determine monitoring needs. One-time surveys are in essence a continuation of the "reconnaissance" studies developed for the PEIR. It is anticipated that focused investigations will be used in conjunction with data collected through the monitoring program to answer key questions and to assist with efforts to adaptively manage restoration activities.

\section{Adaptive Management}

The USGS Tiger Team report of recommendations to the Salton Sea Science Subcommittee (F.S. Shipley, written commun., 1999) notes that "adaptive management" is frequently cited as an effective approach to management of natural systems; however, the term is widely misunderstood and rarely is it actually undertaken. Adaptive management requires feedback between management practices and monitoring of responses in the ecosystem in order to measure the success of management actions and to fine-tune future actions accordingly. In practice, this means that science is used to design the actions, and then monitoring results are provided to managers overseeing restoration so that adjustments can be made, if needed. This feedback works best if scientists are engaged in the design of restoration actions as experiments where outcomes can be predicted and then measured appropriately. Modeling the ecosystem can also benefit from adaptive management principles, where scientists fine tune models on the basis of changes in the systems they are modeling following specific management actions.

Monitoring is a fundamental element of adaptive management, and implementation of this MAP will inform the adaptive management process. In addition to the broad-scale monitoring defined in this MAP, we anticipate that specific monitoring activities intended to test initial assumptions about performance and effectiveness will be incorporated into this MAP as management actions are undertaken.

\section{Data Management}

Data, analyses, and publications developed from this plan will be organized, stored, and made publicly accessible through a common distributed data-management system (that is, CDFW's Biogeographic Information and Observation System, or BIOS). Objectives for data management include 1) defining contract and budget expectations for data management, 2) establishing and enforcing standards for data documentation, 3) establishing data transfer and storage protocols, and 4) making data publicly available and easily accessible in a useable format. Common protocols will be developed and applied when possible, and all geospatial data will include full metadata and will be compliant with the Federal Geographic Data Committee (FGDC) standards. More detailed information regarding data management is in the "Data Management" section of this plan.

The CDFW will establish and maintain the data-management system. The data collected as part of the Salton Sea restoration program will be stored in the CDFW's BIOS map viewer and all documentation including metadata will be accessible to the public via metadata clearinghouses and the CDFW's document library. 


\section{Data Assessment and Analysis}

Data collected and stored within the data management system will be publicly available for the application of statistical and other analytical techniques. This step helps to simplify the collected data, test for change and differences, develop and test hypotheses, and evaluate uncertainty. Data assessment is used to foster the integration, consolidation, and review of data, for updating of the conceptual models, answering of key questions, reporting, and providing management support within an adaptivemanagement framework. Consistent review and assessment of the data are needed to assure that performance objectives are being met and that funding for data collection is effectively utilized. In addition to program-level data assessment and analysis, data assessment should be performed by scientists collecting the data at the individual monitoring activity level through regular evaluation and assessment of data collected over time. This will help ensure data quality and usefulness relative to meeting monitoring objectives.

In cooperation with technical and management staff, and with input from stakeholder groups, the assessment and analysis of data are anticipated to lead to periodic updates of the entire MAP. Updates can be focused on specific resource areas or be applied to the entire plan. The plan is envisioned to be a living document and will need to remain flexible in order to respond effectively to unanticipated events. 


\section{Biological Resources Monitoring}

\section{Overview}

The Salton Sea and its vicinity support a unique ecosystem. Because of its high biological productivity and location on the "Pacific Flyway," the Salton Sea serves as an important wintering area and stopover for thousands of birds during migration (Shuford and others, 2002). Since its formation, the ecosystem of the Salton Sea has been changing in response to the increasing salinity of the Salton Sea. As the Salton Sea ecosystem continues to transition, the aquatic community will become less diverse, and important components of the current system, such as fish, will be lost (Riedel and others, 2002). Changes in the ecosystem not only threaten to reduce habitat suitability for species that inhabit the Salton Sea, they have the potential to affect birds at a regional and international scale. The success of future actions at the Salton Sea to provide or create conditions suitable to support sustainable fish and bird populations will depend on current and reliable information. The intent of this monitoring plan is to provide guidance to collect any additional information needed to serve that purpose.

To develop the technical basis needed for effective decision making and management, the collection of this information through biological monitoring must be performed at the appropriate spatial and temporal scales and include all of the key trophic levels of the ecosystem. Therefore, biological resources monitoring includes several components of the system: birds, fish, invertebrates, and plankton. Monitoring of these ecosystem components, in combination with information collected through monitoring of the non-biological components of the system (for example, water and sediment quality), is expected to contribute data that will aid in understanding the key ecological drivers and processes that determine abundance, distribution, and habitat use in the Salton Sea ecosystem. Monitoring will also inform management decisions, inform the design and operation of created habitats, and provide the basis for development of focused investigations necessary to better manage the resources at the Salton Sea. Ultimately, monitoring data will help to evaluate and document the performance of potential restoration activities.

The individual monitoring activities that could be implemented are described below. For the biological monitoring activities described in this plan, a general description of the expected monitoring protocols (including timing, location, data collection techniques, data analysis, and reporting) is provided. Protocols that were developed with input from experts with familiarity with the biological resources of the Salton Sea area are presented as initial guidance for data collection and are not intended to serve as the exact procedures to be applied. Flexibility remains to refine and revise the protocols as needed prior to implementation; however, changes in the protocols described in the MAP will be documented and peer reviewed.

The resolution needed to meet the monitoring objectives and answer questions related to future management will be predefined. For many of the monitoring activities described below, the sampling variability and other statistical measures needed to estimate confidence intervals and determine statistical power have not been determined. Accordingly, one of the initial purposes of the monitoring activities will be to assess variability. Depending on the specific objectives (for example, determine presence or absence of a fish species), some monitoring activities could only need a single comprehensive "snapshot" of the biological resources of the Salton Sea to provide general guidance and help decision makers, resource managers, and researchers develop and focus more detailed and quantitative monitoring activities and investigations where needed to inform future management. In other instances, the monitoring activity, especially those directly linked to the success of created 
habitats, could require considerably more sampling or survey effort. The elements of the detailed protocols can be refined through the peer review process.

\section{Objectives}

The objectives of the biological resources monitoring activities are directly related to the goals and objectives identified for the MAP and fall into three general categories: 1) ones related to determining the current status of resources, 2) ones establishing a monitoring framework to allow an assessment of future trends in resources; and 3) one addressing the identification, prioritization, and filling of data gaps related to the use of the Salton Sea by biological resources. The current status of biological resources will represent baseline conditions from which changes in status over time can be measured for the abundance, and occupancy or use strata by individual species or groups of species. The retrospective analyses and establishment of existing conditions will be a starting point for comparison with future changes in, as well as historic levels of, use and will provide a basis for evaluating the performance of restoration actions in terms of the stated objectives. The resolution of data gaps will focus on identifying and refining what is known about the ecological relationships and environmental attributes that influence abundance, distribution, and use. Most of these relationships cannot be identified or refined by monitoring alone; however, data describing or measuring environmental attributes collected during monitoring, combined with abundance and occupancy data, can provide insights into relationships that could be further clarified through focused investigation and analysis. The initial objectives for each of the monitoring activities are identified in the sections that address those specific activities.

\section{Key Questions and Information Needs}

As described previously, the overall guidance for this monitoring plan was developed in collaboration with various experts familiar with the Salton Sea area through a process that identified key questions regarding biological resources that, when answered, would provide fundamental information on the response of biological resources to changes in the environment over time. The answers to these questions also would reduce the level of uncertainty regarding the potential success of future restoration actions. Table 1 provides a summary of these questions and information that will be collected through the monitoring activities that will contribute to answering those questions. The information needs to help answer questions regarding trends in use that focus on estimates of biological resources in various areas in and around the Salton Sea. Much of the information related to the environmental attributes and conditions associated with uses will be collected as parts of other elements of the monitoring plan (for example, water quality), however, and coordinated monitoring times among elements can increase the value of the dataset and the information gained from it.

\section{Geographic Scope}

The assessment area for biological monitoring covers the Salton Sea ecosystem, which is defined in Section 2931 of the Fish and Game Code as including, but not limited to "the Salton Sea, the agricultural lands surrounding that sea, and the tributaries and drains within the Imperial and Coachella valleys that deliver water to the Salton Sea."

During development of the proposed biological monitoring element of the MAP, the Salton Sea ecosystem was divided into geographic strata (fig. 5) to help guide monitoring activities and protocols and to ensure that data will be collected from all areas within the Salton Sea ecosystem thought to be important to biological resources. The geographic strata were chosen to be representative of important 
biological components (that is, habitats) and to facilitate monitoring within pre-defined areas. Geographic stratification also can improve the ability to collect and compare data collected through monitoring. The geographic strata in which monitoring will be performed are described in following sections.

Because the monitoring program focuses on collecting data that will be useful for informing decisions regarding potential restoration actions at the Salton Sea, the biological monitoring efforts will initially focus on the Salton Sea itself (shoreline and open-water strata) and the created habitats (for example, created freshwater, including brackish, and saline impoundments) located adjacent to the Salton Sea. Other geographic strata (for example, agricultural lands) will be monitored less intensively. As the monitoring program proceeds, the focus of the monitoring activities could shift to other strata where additional data will aid in understanding ecosystem function and informing management actions.

Specific locations for initial monitoring are described in each element of the biological monitoring plan. To retain consistency among the various monitoring activities, these strata are defined and described in general terms below. Stratification of the assessment area can be modified as the monitoring program is refined over time (that is, new strata may be identified for monitoring). The monitoring activities that can be performed within each of these strata are presented in table 2, which follows the description of geographic strata.

\section{Salton Sea Shoreline}

For the purposes of biological monitoring, the Salton Sea shoreline is defined as the area within a 1-kilometer $(\mathrm{km})$ band around the outer margin of the Salton Sea from 25-meters landward of the land/water interface to 1-kilometer toward the center of the lake. Researchers identified the area extending a distance of about 1 kilometer from the shore as the area of greatest use by birds (for example, Shuford and others, 2000). Similarly, fish in the Salton Sea have been reported to be concentrated in the nearshore and estuarine areas (Riedel and others, 2002). The shoreline stratum also includes the beaches and other features (for example, shoreline pools and barnacle bars) extending from the waterline landward to a distance of 25 meters to include areas used by wading and shore birds. The shoreline stratum also includes the river mouths, other tributaries, and washes that discharge brackish water into the lake. The physical location and area of the shoreline stratum is expected to change as the elevation of the Salton Sea fluctuates seasonally and annually, and monitoring efforts will be adjusted accordingly. At the water-surface elevation of -228 msl used in the Draft PEIR (California Resources Agency, 2006), the area of the shoreline stratum is approximately 43,700 acres $(41,800$ acres within the $1-\mathrm{km}$ band and 1,900 acres with the 25-meter band). The elevation of the lake is anticipated to become lower slowly until around 2017, and then the rate of decline is expected to increase. Therefore, the area of the shoreline stratum will vary through time.

\section{Salton Sea Open Water}

The Salton Sea open-water stratum is defined as that portion of the Salton Sea located inward of the $1-\mathrm{km}$ band that defines the shoreline stratum. This represents the vast majority of the area of the Salton Sea. Open water supports habitat for fish and invertebrates, and the birds that rely on those resources as a forage base. Birds also use the open water for loafing, rafting, and as a staging area prior to migration. Open water also provides birds with protection from most predators and human disturbance. The physical location and area of the open-water stratum will change as the elevation of the Salton Sea fluctuates seasonally and annually and monitoring efforts will be adjusted accordingly. At a water-surface elevation of $-228 \mathrm{msl}$, the area of the open-water stratum is approximately 191,500 acres. The elevation of the lake is anticipated to decline slowly until around 2017, and then the rate of decline 
is expected to increase. Therefore, the area of the open-water stratum will vary through time and is expected to decrease in the future.

\section{Created Freshwater Impoundments}

For the purpose of biological monitoring, the created freshwater-impoundment stratum includes created water bodies that are managed at salinity levels less than $20,000 \mathrm{mg} / \mathrm{L}$, which could include impoundments that would be considered "brackish." The created freshwater-impoundment stratum includes the seasonal and permanent water bodies managed and maintained on the State and Federal refuges located at the Salton Sea and on the Torres Martinez Reservation. These areas include shallow, open-water impoundments managed primarily for waterfowl, densely vegetated impoundments managed for rails and other secretive marsh birds, and impoundments designed for water-quality improvement and general wildlife use.

Two refuge systems are associated with the Salton Sea. The Imperial Wildlife Area (WA) is managed by the CDFW, and the Sonny Bono Salton Sea National Wildlife Refuge is managed by U.S. Fish and Wildlife Service (USFWS). The Imperial WA consists of three separate management units: Wister Unit (5,243 acres), Hazard Unit (639 acres), and Finney-Ramer Unit (2,047 acres). Approximately 4,000 acres of the Imperial WA are managed as seasonally flooded ponds to support waterfowl. These areas typically are flooded in October and remain flooded until late March or April. At the Wister Unit, 700 to 1,500 acres of permanently flooded impoundments are managed for rails.

On the Sonny Bono Salton Sea refuge, about 900 acres are managed as a mosaic of emergent freshwater vegetation and open water. The refuge has an established program to manage about 200 acres of its freshwater marsh area for Yuma clapper rail (and to a lesser extent, California black rail). About 75 acres are managed as brackish water ponds near the Salton Sea shoreline for use by gull-billed terns and black skimmers. The remaining acreage of freshwater marsh is managed as seasonal marsh for use by waterfowl, and they remain flooded from September through March or April. These areas also are flooded for about 6 weeks in the summer to promote the growth of vegetation to provide forage for waterfowl during the fall.

Freshwater impoundments include Finney (310 acres) and Ramer (340 acres) lakes, and the constructed freshwater ponds (22 acres) at the Torres Martinez Reservation. The pilot wetlands on the Torres Martinez Reservation draw water from the Whitewater River near the location where the river meets the Salton Sea. Water from the river is routed through a treatment-wetland system (about 10 acres) intended to improve water quality before it is discharged into a 12-acre freshwater wetland designed to provide wildlife habitat.

\section{Created Saline Impoundments}

For the purpose of biological monitoring, the created saline-impoundment stratum includes created water bodies that are managed at salinity levels greater than $20,000 \mathrm{mg} / \mathrm{L}$. An example of this type of impoundment is the Reclamation/USGS Saline Habitat Ponds (about 119 acres) that were constructed along the southeastern shore of the Salton Sea. The USGS ponds were decommissioned in 2010. This habitat stratum would also include saline ponds constructed at the Salton Sea in the future to support fish and wildlife, as described and evaluated in the PEIR. Newly constructed saline impoundments would be incorporated into the monitoring program as they are constructed. 


\section{Agricultural Land}

Agricultural lands are defined as those areas that support irrigated crops and field conditions that provide one or more life requisites (for example, foraging and roosting) for a variety of wildlife. The extent of use is influenced by the crop type, agricultural practices (for example, irrigation practices), and proximity to other features such as the Salton Sea. The agricultural-land stratum includes actively managed agricultural lands within the IID and Coachella Valley Water District service areas. It also includes areas within the crop matrix that are temporarily or permanently fallowed. The total area of agricultural land within this stratum is currently about 585,000 acres.

\section{Direct Drains}

Drainage from agricultural lands is conveyed to the Salton Sea through a network of drains. For the purposes of this monitoring plan, the direct-drain stratum is defined as the watered portion of the drains that empty directly into the Salton Sea from the point they discharge into the Salton Sea upstream to the first pump or check structure that could inhibit movement of fish into the upper reaches of the drain. These portions of the drains are directly linked to the Salton Sea and support aquatic species, such as desert pupfish, that move between these two water bodies. All drains and ponds (for example, Lack and Lindsay Pond) with a direct connection to the Salton Sea in the Imperial Valley and in the Coachella Valley compose the direct-drain stratum for purposes of this monitoring program.

\section{Halophytic Scrub}

Halophytic scrub includes areas around the margin of the Salton Sea that support halophytic vegetation. These areas are adjacent to portions of the Salton Sea shoreline defined previously and support tamarisk and other halophytic shrubs (for example, arrowweed). Halophytic scrub vegetation adjacent to the Salton Sea is generally restricted to areas where there is sufficient soil moisture (usually from irrigation seepage from adjacent parcels) in a thin band around the perimeter of the lake, which is referred to as shoreline strand. The vegetation is expected to change as the elevation of the lake decreases over time, and it could encroach onto the exposed playa as the lake recedes. Areas of halophytic scrub can support roosting and nesting shorebirds and provide cover for passerine birds during migration. During surveys performed within $1 \mathrm{~km}$ of the Salton Sea, Mendelsohn and others (2006) recorded 44 landbird and shorebird species apparently associated with halophytic scrub. They found that both bird abundance and species diversity were greatest at survey locations nearest the Salton Sea.

For the purpose of this monitoring plan, the halophytic scrub stratum includes all stands of halophytic shrubs located within $1 \mathrm{~km}$ of the Salton Sea. This area contains the portion of halophytic scrub that supports the greatest bird diversity and abundance, which is likely strongly influenced by its proximity to the Salton Sea. As the Salton Sea recedes, it is anticipated that the location and area of this stratum will change and monitoring activities will be adjusted accordingly.

\section{Conceptual Model}

The key questions identified previously were developed, in part, on the basis of a conceptual model for biological resources in the Salton Sea ecosystem (fig. 6). The conceptual model includes a simplified food-web diagram for the Salton Sea, identifies the species groups that use the various geographic strata, and identifies the linkages between geographic strata for the species groups. This conceptual model serves as the starting point for refining the understanding of how the system functions, where there are data gaps, and where additional data collection would reduce uncertainty. 
This conceptual model could be modified over time as monitoring activities increase the understanding of how the system functions.

\section{Avian Resources Monitoring Activities}

The monitoring of avian resources focuses on birds using the Salton Sea itself and the immediately adjacent areas. The objective of these monitoring activities is to document the species composition, relative abundance, spatial and temporal distribution, and broad-scale habitat associations of bird species that use the Salton Sea. While it is possible the information gathered would not allow for the determination of whether observed changes in abundance, species composition, and distribution were statistically significant, the initial surveys are expected to provide a coarse-level screening of bird use at the Salton Sea. They also will allow the determination of sample and survey variability that can be used to determine confidence intervals around abundance estimates and to estimate the number of samples or surveys that are required to provide adequate statistical power to meet specific monitoring objectives within the adaptive management framework.

It is anticipated that the survey results will allow researchers and decision makers to draw preliminary conclusions about changes over time that could guide restoration activities and the design or management of created habitats. The survey results also will help to assess the validity of assumptions in conceptual models and determine if focused investigations are needed to refine conceptual models and answer key questions regarding avian resources at the lake. The specific objectives of the avian resources monitoring activities and their intended purpose are presented in table 3.

To enhance the value of these activities, in addition to counts of each species, the location of each observation of birds or groups of birds would be recorded in a manner that is as spatially explicit as practical. This provides the opportunity to detect patterns of use that would not be otherwise apparent. This level of spatial resolution also would allow for a GIS evaluation of bird use relative to environmental attributes.

Analysis of the monitoring data collected could possibly not include every species detected because of limited observations of certain transient or rarely encountered species. With respect to avian resources, analysis could be limited to those that have a special status designation or use the Salton Sea in numbers that represent significant segments of their regional or North American populations. Following an approach similar to that used in the Draft PEIR (California Resources Agency, 2006), focal species can include those listed as state or federally "Threatened" or "Endangered" species on the CDFW Bird Species of Special Concern list, species considered "Highly Imperiled" or of "High Concern" under the National Waterbird Conservation Plan, species or subspecies with a 4-5 priority score under the U.S. Shorebird Conservation Plan, species on the USFWS Birds of Conservation Concern - BCR 33 list, or species of which more than 10,000 birds were counted on a single survey at the Salton Sea.

The monitoring activities described in this document were identified and organized by grouping activities with similar protocols, species, or geographic areas to improve the efficiency with which they can implemented. Although these activities could be performed independently, close coordination ensures consistency and integration. It is most beneficial when the entire suite of avian monitoring activities is performed each year because the assessment value of individual elements is minimal without data from the other elements.

Surveys of avian resources within the shoreline and open-water strata at the Salton Sea and in created saline and freshwater impoundments would be coordinated with other avian resource surveys to allow for development of an estimate of the abundance and spatial distribution of birds using the Salton Sea on an annual and seasonal basis during the assessment portion of the MAP. Different elements of 
the avian resources monitoring performed on approximately the same days efficiently achieves the intent that results be combined into a single estimate of bird use and describe the distribution of bird use of the entire Salton Sea area. It is possible that individual birds can be counted in more than one stratum during coordinated monitoring activities. The assessment portion of the MAP will include methods to reconcile these counts and develop the best estimate of overall bird abundance at the Salton Sea. Avianmonitoring activities under the MAP also could be coordinated with the ongoing monitoring activities performed by others (for example, CDFW, USFWS, L.A. County Natural History Museum, USGS, Torres Martinez) in the Salton Sea area.

\section{Bird Surveys (Salton Sea Shoreline)}

The following sections describe the annual monitoring activities needed to meet the monitoring objectives for bird species that primarily use shoreline areas at the Salton Sea. Results of this monitoring activity, in combination with other bird surveys, could be used to establish and characterize the current use of the Salton Sea by birds. Data collected from this activity (and other elements of the MAP) could be used to identify trends in use of the Salton Sea by bird species and the environmental attributes and conditions that influence their use.

\section{Purpose and Justification}

Bird surveys of the Salton Sea shoreline are necessary to establish the existing conditions for bird use (numbers and distribution), identify trends in bird use of shoreline areas, and to address key questions related to bird use at the Salton Sea. The data collected during the surveys also would help refine the understanding of bird distribution and use along the Salton Sea shoreline and qualitatively describe the dominant habitats and habitat features used by birds.

The Salton Sea shoreline and shallow water areas are anticipated to change substantially with reductions in water-surface elevation and changes in water quality. Developing a greater understanding of trends in bird use as this area changes would provide information to help guide management actions directed toward continued bird use of shoreline habitats. This element of the avian-resources monitoring could provide important insights into the physical and biological characteristics of the shoreline area that affects bird use and could help ensure that any future habitat creation is constructed in a manner that maximizes the benefits to the birds that rely on these areas. The monitoring data also can identify relationships between bird use and environmental conditions that could lead to the development of focused investigations that test hypotheses about those relationships.

\section{Location}

To obtain data on the abundance and patterns of distribution and habitat use of birds using the shoreline of the Salton Sea, the survey area (shoreline stratum) could be divided into segments of similar environmental characteristics spanning the entire shoreline of the lake. To the extent appropriate, these areas will be chosen for compatibility with earlier surveys (Shuford and others, 2000) to help ensure comparability to previous results. Sampling locations within each shoreline segment would be randomly selected to ensure that survey data are representative of the shoreline and associated features within the shoreline stratum. On the basis of results of the retrospective analysis, this stratum could be further subdivided to ensure adequate sampling in areas known to support greater bird use (for example, river mouths). 


\section{Period and Frequency}

Bird surveys of the Salton Sea shoreline are proposed to be initially performed for at least 3 years, after which the need and scale of monitoring can be re-evaluated. Each year, surveys could be performed during the late winter, spring migration, breeding season, early fall, and early winter. Specific seasons and dates of surveys will be established in consultation with the Focused Technical Group and other experts. Multiple observations at selected shoreline locations will be performed during each season to assess variability.

Because results of this and avian surveys in other geographic strata are intended to be combined into a single estimate of bird use and describe the distribution of bird use of the entire Salton Sea area, elements of the avian resources monitoring will require coordination between groups performing the various avian monitoring activities in other strata. Avian monitoring activities under the MAP also could be coordinated with the ongoing avian monitoring activities performed by others in the Salton Sea area.

\section{Protocol and Data Collection}

The survey protocol and data-collection techniques will be based, in part, on the 1999 Comprehensive Waterbird Surveys at the Salton Sea (Shuford and others, 2000) and be designed to provide an estimate of the number of birds using the shoreline areas (within $1.0 \mathrm{~km}$ of the shoreline and 25-m inland) of the Salton Sea on the days of the surveys. It is anticipated that the surveys could be performed from the water by positioning a boat at pre-selected survey locations within the shoreline stratum that provide good vantage points for observing birds and recording all birds observed within reasonable viewing range. Alternative methods (use of airboats, personal watercraft) or techniques (aerial surveys or photography) can be used as appropriate to adapt to changing conditions at the lake. Survey locations will be determined prior to field work each year and selected to be representative of the shoreline segment and that include single features (for example, snags or islands) and other environmental attributes (for example, substrate or vegetation type) thought to be important for bird use.

Although most observations will be made from the water, it is anticipated that some shoreline segments could be surveyed from the shore. The coordinates of each location where observations (boat or shore position) are made will be recorded by using GPS. All birds observed will be identified to the species level (or lowest taxon possible) and enumerated. In addition, approximate locations where bird use is concentrated will be identified to the extent practical by using GPS, a combination of GPS and compass bearings (and distance determined by using a precision rangefinder) from known locations, or other reliable means. In addition to observations of bird use (species, numbers, and spatial distribution), habitat features (such as islands, isolated snags, levees, pilings and platforms, exposed rocky reefs, exposed sandbars, and shoreline pools) within shoreline segments with areas of concentrated use will be identified, and their location recorded, during the surveys. Weather conditions, including ambient temperature, wind speed, wind direction, and sky condition at each survey point, will be recorded at the time of the survey.

Prior to sampling, conceptual models and working hypotheses will be developed that identify the environmental attributes that are most likely to influence bird use of shoreline areas. The development of these environmental attributes should be performed in coordination with other monitoring activities to help ensure consistency. The selected environmental attributes and how they correspond to survey locations will be described in the detailed monitoring protocol prepared prior to initiation of sampling. This information helps establish correlates of bird use with other ecological variables. 


\section{Data Analysis}

Surveys of birds within the shoreline stratum will be used to describe bird use (species, number, and distribution) on a seasonal and annual basis at the Salton Sea and to assess changes over the duration of the MAP. It is anticipated that the data collected on the location of areas of concentrated use will be used in conjunction with other monitoring results (for example, the location and number of habitat features) collected during the shoreline- bird surveys and information on other environmental variables collected through other elements of the monitoring plan to identify important ecological variables that affect bird use at the Salton Sea. The information gained from this monitoring activity is anticipated to guide management at the Salton Sea, including focused investigations and possibly the construction and management of alternative habitats, such as created saline habitats.

\section{Quality Assurance}

As described previously, a detailed monitoring protocol will be developed prior to initiating monitoring activities in the field. This protocol will include a description of the measures that ensure the quality of the data collected and how to implement those measures. The data quality-assurance measures can include, but are not limited to, procedures for calibrating or ensuring the accuracy of any instruments (for example, GPS) employed in the field, procedures for recording and transferring electronic data, methods for ensuring proper operation of field equipment during surveys, and methods for avoiding double counting or insufficient coverage of survey areas. Data-quality protocols also could take into consideration the influence of weather conditions on bird detections and counts and include measures to minimize weather-related effects.

\section{Data Reporting}

Data will be archived and made accessible from the CDFW's BIOS database when appropriate. Data submitted to the CDFW must be provided in a format suitable for inclusion in the BIOS database (see the "Data Management" section), including appropriate metadata. In addition, an annual progress report will summarize the data collected during that year and update prior reports in a cumulative fashion. At a minimum, the annual report can include the following elements:

- An estimate of the annual and seasonal abundance of all birds using shoreline areas at the Salton Sea, including confidence intervals around all estimates, where appropriate, and methods used to generate estimates of variance.

- Species composition and relative abundance of birds observed using shoreline areas at the Salton Sea.

- Distributional patterns of bird use along the shoreline of the Salton Sea (that is, mapped locations of observations) and relative abundance (number observed) at each location identified during the surveys.

- Environmental information collected during the shoreline monitoring.

- Recommendations for improvement of the survey protocol, if any. 


\section{Overview of Similar Current Monitoring Activities}

CDFW and USFWS currently perform point counts of waterbirds along the shoreline that could supplement this monitoring activity.

\section{Bird Surveys (Salton Sea Open Water)}

The following sections describe the annual monitoring activities needed to meet the monitoring objectives for bird species that primarily use open-water areas at the Salton Sea. Results of this monitoring activity, in combination with other bird surveys, will be used to establish and characterize the current use of the Salton Sea by birds. Data collected from this activity (and other elements of the MAP) will be used to identify trends in use of the Salton Sea by bird species and the environmental attributes and conditions that influence their use.

Purpose and Justification

Bird surveys of the open-water portion of the Salton Sea are necessary to establish the existing conditions for bird use (numbers and distribution), identify trends in bird use of open-water areas at the Salton Sea, and to address key questions related to bird use at the Salton Sea. Ongoing CDFW aerial surveys have concentrated on enumerating cormorants and pelicans that use open-water areas. Aerial waterfowl surveys performed by USFWS do not currently include offshore areas. In addition, there are challenges in identifying birds to the species level during aerial surveys and discriminating birds from other floating objects. Boat-based surveys across the open-water portion of the Salton Sea could allow for improved identification of waterbirds and provide the spatial coverage necessary for an accurate picture of bird use in the open-water portion of the Salton Sea.

The creation of deeper, open-water areas is a consideration for potential restoration activities at the Salton Sea. This monitoring activity will also provide information that could be useful in designing and managing water bodies to benefit specific bird species.

Locations

To obtain data on the abundance and patterns of distribution in bird use on the open waters (farther than $1.0 \mathrm{~km}$ offshore) of the Salton Sea, the surveys will be performed along transects within the open-water areas of the Salton Sea. An arrangement of transects covering the entire open-water stratum will be developed prior to sampling. Transects included in the survey will be selected to ensure that survey data are representative of the open-water stratum. .

\section{Period and Frequency}

Bird surveys of the Salton Sea open water initially are proposed to be performed for at least 3 years to capture annual variation, after which the need and scale of monitoring can be re-evaluated. Each year, surveys could be performed during the late winter, spring migration, breeding season, early fall, and early winter. Specific seasons and dates of surveys will be established in consultation with the Focused Technical Group and other experts. Multiple surveys will be performed along open-water transects during each season for an assessment of variability.

Because results of this and avian surveys in other geographic strata are intended to be combined into a single estimate of bird use and describe the distribution of bird use of the entire Salton Sea area, elements of the avian-resources monitoring needs to be performed on approximately the same day(s). This requires coordination among groups performing the various avian monitoring activities in other 
strata. Avian monitoring activities under the MAP also should be coordinated with the ongoing avian monitoring activities carried out by other groups in the Salton Sea area.

\section{Protocol and Data Collection}

It is anticipated that all surveys will be performed by using traditional motor boats, although other forms of watercraft (for example, personal watercraft) can be used as appropriate. Alternative techniques (aerial surveys or photography) can be used as conditions change at the lake. Each survey will consist of observations along a sufficient number of transects to provide adequate spatial coverage of the stratum. All birds observed within a band approximately $300 \mathrm{~m}$ on each side of survey transects will be identified to the lowest taxon possible and enumerated to estimate bird abundance and describe species composition for individual transects and the open-water stratum as a whole. Transects will be followed by using GPS; approximate locations where bird use is concentrated will be identified to the extent practical by using GPS, a combination of GPS and compass bearings (and distance determined by using a precision rangefinder) from known locations, or other reliable means. Placement of buoys or other floating markers prior to surveys could be necessary to provide points of reference for observers in the field. Weather conditions, including ambient temperature, wind speed, wind direction, and sky condition at each survey point will be recorded at the time of the survey.

\section{Data Analysis}

The bird observations recorded within the open-water stratum will be used to describe seasonal and annual bird use (species, number, and distribution) at the Salton Sea and to assess changes during the MAP. Survey results could be used in conjunction with other information to identify important environmental attributes that affect bird use of open water areas at the Salton Sea. The information gained from this monitoring activity is anticipated to guide management at the Salton Sea, including the development of focused investigations and possibly the construction and management of alternative habitats, such as created saline habitats.

\section{Quality Assurance}

As described previously, a detailed monitoring protocol and sampling design will be developed prior to initiating monitoring activities in the field. This protocol will include a description of the measures that ensure the quality of the data collected and how to implement those measures. The data quality-assurance measures can include, but are not limited to, procedures for calibrating or ensuring the accuracy of any instruments (for example, GPS) employed in the field, procedures for recording and transferring electronic data, methods for ensuring proper operation of field equipment during surveys, and methods for avoiding double counting or insufficient coverage of survey areas.

\section{Data Reporting}

Data will be archived and made accessible from the CDFW's BIOS database when appropriate. Data submitted to the CDFW must be provided in a format suitable for inclusion in the BIOS database (see the "Data Management" section), including appropriate metadata. In addition, an annual progress report will summarize the data collected during that year and update prior reports in a cumulative fashion. At a minimum, the annual report can include these elements:

- An estimate of the annual and seasonal abundance of birds observed using open water areas at the Salton Sea, including confidence intervals around all estimates, where appropriate, and methods used to derive estimates of variance. 
- Species composition and relative abundance of birds observed using open water of the Salton Sea.

- Distributional patterns of bird use within the open water portion of the Salton Sea (that is, mapped locations of observations) and relative abundance (number observed) at each location identified during the surveys.

- Recommendations for improvement of the survey protocol, if any.

Overview of Similar Current Monitoring Activities

The CDFW and USFWS currently perform aerial counts of selected waterbirds and waterfowl that may supplement this monitoring activity.

\section{Bird Surveys (Created Saline Impoundments)}

The following sections describe the annual monitoring activities needed to meet the monitoring objectives for bird species that use created saline impoundments around the Salton Sea. Results of this monitoring activity, in combination with other bird surveys, will be used to determine and characterize the current use of the Salton Sea by birds. Data collected from this activity (and other elements of the MAP) could be used to identify trends in use of saline impoundments by bird species and the environmental attributes and conditions that influence their use of these areas.

\section{Purpose and Justification}

Created saline impoundments (salinity previously $20,000 \mathrm{mg} / \mathrm{L}$ ) include water bodies created for the purpose of providing wildlife habitat, improving water quality, or habitat experimentation near and adjacent to the Salton Sea. These impoundments generally are sparsely vegetated and provide important foraging areas for many birds associated with the Salton Sea area that feed on aquatic invertebrates and fish.

Bird surveys at selected saline impoundments are necessary to establish the existing conditions for bird use (numbers and distribution), identify trends in bird use of saline impoundments, and to address key questions related to bird use at the Salton Sea area. These surveys provide a more precise estimate of overall bird use, including bird species that move back and forth between near-lake impoundments and the lake itself. The survey results are expected to provide information that allows researchers to draw initial conclusions about changes in bird use over time and guide potential restoration activities and the design and management of created habitats.

Locations

To obtain data on the abundance and patterns of distribution and habitat use of birds around the Salton Sea, several created saline impoundments adjacent to the Salton Sea could be randomly selected for annual monitoring. Survey locations could be chosen to represent a range of conditions (for example, depth, substrate, salinity). Impoundments included in the surveys could be located on proximate state, federal, tribal, or private lands.

\section{Period and Frequency}

Bird surveys in created saline impoundments initially are proposed to be performed for at least 3 years, after which the need and scale of monitoring can be re-evaluated. Each year, surveys could be 
performed during the late winter, spring migration, breeding season, early fall, and early winter. Specific seasons and dates of surveys will be established in consultation with the Focused Technical Group and other experts. Multiple observations at selected impoundments will be performed during each season for an assessment of variability.

Because results of this and avian surveys in other geographic strata are intended to be combined into a single estimate of bird use and describe the distribution of bird use of the entire Salton Sea area, elements of the avian resources monitoring need to be performed on approximately the same days. This requires coordination among groups performing the various avian monitoring activities in other strata. The monitoring of birds at created saline impoundments will be closely coordinated and integrated with results of work performed on state, federal, tribal, and private lands.

\section{Protocol and Data Collection}

Because these impoundments generally have large areas of open water and good visibility, observers could record the number and species of all birds within view from the margins of the impoundments. These observations will be made at established locations over a specified time span, and the GPS coordinates for each observation station will be recorded. In addition to observations of bird species, observers will record habitat characteristics (for example, size, shape, depth, presence of features) at each of these impoundments. Weather conditions, including ambient temperature, wind speed, wind direction, and sky condition at each impoundment will be recorded at the time of the survey. Data on water conditions, such as approximate water level and salinity, will also be recorded at the time of the survey.

Prior to sampling, conceptual models and working hypotheses will be developed to identify the environmental covariates that are most likely to influence bird use of created saline impoundments. The development of these conceptual models could be performed in coordination with other monitoring activities to help ensure consistency. The selected environmental attributes and how they correspond to survey locations will be described in the detailed monitoring protocol prepared prior to initiation of sampling. Characteristics of each impoundment that vary minimally, such as area, pond position (initial or terminal), and proximity to agriculture and the shoreline of the Salton Sea, could be available from other sources. Other characteristics, such as average water depth, water-management status (filling or draw down), and substrate, will be obtained onsite within 1 week of the initial survey, preferably at the time of the survey. Habitat features that potentially influence the use of the impoundments by birds, such as islands, isolated snags, levees, and pilings and platforms, will be noted during the surveys.

\section{Data Analysis}

Observations of waterbirds will be used to describe seasonal and annual bird use (species, number, and distribution) at the created saline impoundments and to assess changes during the MAP. The information gained from this monitoring activity is anticipated to guide management at the Salton Sea, which could include the development of focused investigations and the construction and management of other saline habitats.

\section{Quality Assurance}

As described previously, a detailed monitoring protocol will be developed prior to initiating monitoring activities in the field. This protocol will include a description of the measures that ensure the quality of the data collected and how to implement those measures. The data quality-assurance measures can include, but are not limited to, procedures for calibrating or ensuring the accuracy of any instruments (for example, GPS) employed in the field, procedures for recording and transferring 
electronic data, methods for ensuring proper operation of field equipment during surveys, and methods for avoiding double counting or insufficient coverage of survey areas.

\section{Data Reporting}

Data will be archived and made accessible from the CDFW's BIOS database when appropriate. Data submitted to the CDFW must be provided in a format suitable for inclusion in the BIOS database (see the "Data Management" section), including appropriate metadata. In addition, an annual progress report will summarize the data collected during that year and update prior reports in a cumulative fashion. At a minimum, the annual report can include the following elements:

- An estimate of the annual and seasonal abundance of birds using created saline impoundments adjacent to the Salton Sea, including confidence intervals around all estimates, where appropriate, and methods used to derive estimates of variance.

- Species composition and relative abundance of birds observed using created saline impoundments.

- Environmental information collected during the monitoring (for example, salinity).

- Recommendations for improvement of the survey protocol, if any.

\section{Overview of Similar Current Monitoring Activities}

The USGS performed a variety of monitoring and research activities at their experimental saline ponds that were located near the Salton Sea, some of which addressed bird use. The Torres Martinez Tribe has used volunteer observers to monitor monthly bird use at their pilot wetland project since 2007.

\section{Bird Surveys (Created Freshwater Impoundments)}

The following sections describe the annual monitoring activities needed to meet the monitoring objectives for bird species that primarily use freshwater impoundments around the Salton Sea. Results of this monitoring activity, in combination with other bird surveys, can be used to establish and characterize the current use of the Salton Sea by birds. Data collected from this activity (and other elements of the MAP) could be used to identify trends in use of freshwater impoundments by bird species and the environmental attributes and conditions that influence their use of these areas.

\section{Purpose and Justification}

Created freshwater impoundments include water bodies created for the purpose of providing wildlife habitat, improving water quality, or habitat experimentation near and adjacent to the Salton Sea. These impoundments range in vegetation coverage from those that are heavily vegetated and provide habitat for secretive marsh birds (for example, rails) to those that are sparsely vegetated and managed for waterfowl. These areas provide important habitat for many birds associated with the Salton Sea area, and they serve as a source of freshwater, which is used by some species that frequently inhabit the saline environment of the Salton Sea.

Bird surveys at freshwater impoundments are necessary to establish the existing conditions for bird use (numbers and distribution), identify trends in bird use of freshwater impoundments, and to address key questions related to bird use at the Salton Sea area. These surveys would provide a good estimate of overall bird use, including bird species that move back and forth between near-lake 
impoundments and the lake itself. The survey results are expected to provide information that allows researchers to draw initial conclusions about changes in bird use over time and guide restoration activities and the design or management of created habitats.

\section{Locations}

To obtain data describing the abundance and patterns of distribution and habitat use of birds around the Salton Sea, several freshwater impoundments adjacent to the Salton Sea could be randomly selected for annual monitoring. Because the impoundments vary in terms of depth, vegetative-cover type and density, and level of salinity (brackish waters), this geographic stratum could be further subdivided into impoundments with similar characteristics. Impoundments included in the surveys can be chosen to represent a range of conditions (for example, depth, substrate, vegetative-cover type and density, and salinity) and status (seasonally flooded or permanently). Impoundments included in the surveys can be located on adjacent state, federal, tribal, or private lands.

\section{Period and Frequency}

Bird surveys in the created freshwater impoundments initially are proposed to be performed for at least 3 years, after which the need and scale of monitoring can be re-evaluated. Each year, surveys could be performed during the late winter, spring migration, breeding season, early fall, and early winter. Specific seasons and dates of surveys will be established in consultation with the Focused Technical Group and other experts. Multiple observations at selected impoundments will be performed during each season to assess variability.

Because results of this and avian surveys in other geographic strata are intended to be combined into a single estimate of bird use and describe the distribution of bird use of the entire Salton Sea area, elements of the avian resources monitoring need to be performed on approximately the same days. This requires coordination among groups performing the various avian monitoring activities in other strata. Avian monitoring activities under the MAP also can be coordinated with the ongoing avian monitoring activities performed by others in the Salton Sea area. It is possible to coordinate the freshwater impoundment surveys with the state and federal surveys at the refuges.

\section{Protocol and Data Collection}

Two different survey protocols can be used, depending on the amount and type of vegetation present at the selected impoundment. At some impoundments, both protocols could be applied. At impoundments that have large areas of open water (for example, seasonally flooded impoundments) and good visibility, observers will record the number and species of all waterbirds within view from the margins of the impoundment. These observations will be made at established locations over a specified period of time, and the GPS coordinates for each observation station will be recorded. In addition to observations of bird species, habitat characteristics (for example, size, shape, depth, substrate, vegetative-cover type and density, or presence of features) of these impoundments will be recorded.

At densely vegetated impoundments where vegetative conditions can provide habitat for secretive marsh birds, survey methods and protocols will be used that conform to the standard procedures described in the Standardized North American Marsh Bird Monitoring Protocols (Conway, 2008). These protocols consist of broadcast calls to elicit vocalizations during vocal surveys. Species that respond to the call sequence include, but are not limited to, California black rail, Yuma clapper rail, and least bittern. Surveys can use the double-observer method to estimate detection probability. The GPS coordinates for each call station and the approximate location of each response will be recorded. 
The appropriate survey protocol will be determined through consultation with the Focused Technical Group and other experts. Regardless of the protocol applied, weather conditions, including ambient temperature, wind speed, wind direction, and sky condition at each survey point, will be recorded at the time of the survey. Data on water conditions, such as approximate water level and salinity, will also be recorded at the time of the survey. Information on vegetative characteristics (percentage cover by wetland plants and major vegetation types) will be recorded at a time that overlaps the breeding season (for all of the target marsh birds) and during the winter flood-up period in open impoundments. The estimates of vegetative cover made in the field will be verified through comparison with recent aerial photographs, when available. In addition, the boundaries of each selected impoundment will be clearly identified in a digital format.

Prior to sampling, conceptual models and working hypotheses will be developed to identify the environmental covariates that are most likely to influence birds using created freshwater impoundments. The development of these environmental attributes would be performed in coordination with other monitoring activities to help ensure consistency. The selected environmental attributes and how they correspond to survey locations will be described in the detailed monitoring protocol prepared prior to initiation of sampling. Characteristics of each impoundment that vary minimally, such as area, pond position (initial or terminal), and proximity to agriculture and the shoreline of the Salton Sea, could be available from other sources. Other characteristics, such as average water depth, water-management status (filling or draw down), and substrate, can be obtained onsite within 1 week of the initial survey, preferably at the time of the survey. Habitat features that potentially influence the use of the impoundments by birds, such as islands, isolated snags, levees, and pilings and platforms, will be noted during the surveys.

\section{Data Analysis}

Observations of waterbirds and responses to calls by secretive marsh birds can be used to describe bird use (species, number, and distribution) at the created freshwater impoundments on a seasonal and annual basis and to assess changes over the duration of the MAP. The information gained from this monitoring activity is anticipated to guide management at the Salton Sea, which could include the development of focused investigations and the construction and management of other freshwater habitats. This information also could help to clarify the importance of the proximity of freshwater to birds that use saline water bodies and how freshwater could be incorporated into saline habitat designs for potential restoration.

\section{Quality Assurance}

As described previously, a detailed monitoring protocol will be developed prior to initiating monitoring activities in the field. This protocol will include a description of the measures that ensure the quality of the data collected and how to implement those measures. The data quality-assurance measures can include, but are not limited to, procedures for calibrating or ensuring the accuracy of any instruments (for example, GPS) employed in the field, procedures for recording and transferring electronic data, methods for ensuring proper operation of field equipment during surveys, and methods for avoiding double counting or insufficient coverage of survey areas.

\section{Data Reporting}

Data will be archived and made accessible from the CDFW's BIOS database when appropriate. Data submitted to the CDFW must be provided in a format suitable for inclusion in the BIOS database (see the "Data Management" section), including appropriate metadata. In addition, an annual progress 
report will summarize the data collected during that year and update prior reports in a cumulative fashion. At a minimum, the annual report can include the following elements:

- An estimate of the annual and seasonal abundance of birds using freshwater impoundments adjacent to the Salton Sea, including confidence intervals around all estimates, where appropriate, and methods used to derive estimates of variance.

- Species composition and abundance of birds observed using created freshwater impoundments

- Environmental information collected during the monitoring (for example, salinity).

- Recommendations for improvement of the survey protocol, if any.

Overview of Similar Current Monitoring Activities

The CDFW and USFWS currently perform mid-winter aerial counts of waterfowl on the refuges and duck clubs. The USFWS currently performs annual surveys for Yuma clapper rail on the refuge, and the CDFW currently performs annual surveys for Yuma clapper rail on the Wister Unit of the Imperial WA. In addition, rail/marsh bird surveys are performed periodically by the BLM (using contractors) at the Dos Palmas Preserve and nearby wetlands. These surveys could supplement this monitoring activity.

\section{Colonial Breeding Bird Surveys}

The following sections describe the annual monitoring activities required to establish existing conditions and trends for breeding populations of colonial birds (herons, egrets, ibises, and cormorants, gulls, terns, and skimmers) and their use of breeding sites at the Salton Sea.

Purpose and Justification

The Salton Sea serves as an important breeding area for many colonially breeding birds, and trends in breeding bird numbers and nesting success could provide an indication of the status of these birds at the Salton Sea and the health of the ecosystem. In addition, information about the characteristics of the areas used by breeding birds could be valuable in the planning, design, and management of habitat potentially created in the future. The colonial breeding bird surveys can describe nesting areas, estimate the number of breeding pairs, and provide information on the phenology of nesting at the Salton Sea.

Locations

Monitoring locations will be based on past survey results and reconnaissance-level surveys to locate breeding colony locations (see Overview of Similar Current Monitoring Activities section that follows). All colonies could subsequently be mapped and monitored for breeding activity, species composition of nesting birds, phenology, and general colony success. Breeding colonies could be located in the shoreline and halophytic scrub geographic strata.

Period and Frequency

Colonial breeding bird surveys initially are proposed to be performed for at least 3 years, after which the need and scale of monitoring can be re-evaluated. Each year, surveys could be performed 
between February and September, depending on the species. Specific seasons and dates of surveys will be established in consultation with the Focused Technical Group and other experts. Multiple observations at colonial nesting areas will be performed annually to provide information on phenology and general colony success and to assess variability.

The monitoring of colonial breeding birds at the Salton Sea will be closely coordinated and integrated with the ongoing work of the L.A. County Natural History Museum to avoid duplication and to ensure that the information is collected in a manner that meets the objectives of both programs. This monitoring activity also could include coordination with any ongoing monitoring efforts carried out by the CDFW or USFWS.

\section{Protocol and Data Collection}

Aerial reconnaissance surveys that circumnavigate the Salton Sea could be performed to locate breeding colonies. Surveys performed from January to March locate great blue heron and double-crested cormorant colonies, and in May, they locate later-nesting herons and egrets. The aerial surveys to locate colonies can be followed by ground, boat-based, or aerial photography surveys to estimate the number of breeding pairs.

All colonial nesting birds observed will be identified to the species level and enumerated at individual colonies. In addition to counts of nesting birds, habitat characteristics (for example, degree of isolation, distance from fresh water, substrate) of the colonies will be recorded at the time of the survey, preferably by using non-intrusive methods (for example, aerial photography). Weather conditions, including ambient temperature, wind speed, wind direction, and sky condition at each survey point, will be recorded at the time of the survey. This information can be used to establish correlates of bird distribution with other ecological variables during the assessment phase of the MAP. The location of each colony can be digitally mapped.

Because of the extreme conditions at the Salton Sea, the reproductive success of colonial breeding birds can be strongly influenced by events (such as investigator disturbance) that subject eggs and chicks to exposure and extreme heat. Under these conditions, even relatively short periods away from the nest by adult birds can result in mortality. Given the inherent risks associated with surveying nesting birds under these conditions, the benefits associated with the surveys will be cautiously weighed against the effects of increased investigator disturbance to the colonies. The detailed protocol will aim to minimize disturbance of the breeding colonies.

\section{Data Analysis}

Observations of colonially nesting birds can be used to describe bird use (number of breeding pairs) at the colonies on an annual basis and to assess changes over the duration of the MAP. Repeat surveys (if it is determined that they can be performed without significant disturbance) can provide information on the phenology and general breeding success at the colonies. This information can be used in conjunction with other information, such as the degree of isolation recorded at the time of the surveys, and information on other physical and environmental variables collected by other monitoring teams, to identify important ecological variables that affect the breeding success of colonial waterbirds at the Salton Sea. The information gained from this monitoring activity is anticipated to guide management activities at the Salton Sea, including the development of focused investigations and possibly the integration of nesting structures into created habitats. 


\section{Quality Assurance}

As described previously, a detailed monitoring protocol will be developed prior to initiating monitoring activities in the field. This protocol will include a description of the measures that ensure the quality of the data collected and how to implement those measures. The data quality-assurance measures can include, but are not limited to, procedures for calibrating or ensuring the accuracy of any instruments (for example, GPS) employed in the field, procedures for recording and transferring electronic data, methods for ensuring proper operation of field equipment during surveys, and methods for avoiding double counting or insufficient coverage of survey areas.

\section{Data Reporting}

Data will be archived and made accessible from the CDFW's BIOS database when appropriate. Data submitted to the CDFW must be provided in a format suitable for inclusion in the BIOS database (see the "Data Management" section), including appropriate metadata. In addition, an annual progress report will summarize the data collected during that year and update prior reports in a cumulative fashion. At a minimum, the annual report is to include the following:

- GIS maps showing locations of breeding colonies.

- An estimate of the annual abundance of colonial birds breeding at the Salton Sea, including confidence intervals around the abundance estimate, where appropriate, and the methods used to derive estimates of variance.

- Species composition and relative abundance of colonial birds breeding at the Salton Sea.

- Phenology and general colony success of colonial birds breeding at the Salton Sea (if it is determined that repeat surveys can be performed without significant disturbance).

- Environmental information collected in conjunction with the colonial breeding bird surveys

- Recommendations for improvement of the survey protocol, if any.

Overview of Similar Current Monitoring Activities

The Los Angeles County Natural History Museum has performed long-term annual monitoring of breeding larids (gulls and terns) at the Salton Sea since 1992 (Molina, 2004). In addition, breeding herons, egrets, cormorants, and ibises were monitored annually by the USFWS (Sonny Bono National Wildlife Refuge) between 1986 and 1999 (Molina and Sturm, 2004). Additional breeding bird surveys are anticipated to be carried out by the USFWS and Point Reyes Bird Observatory (PRBO).

\section{Bird Counts at Roost Sites}

The following sections describe annual monitoring activities that could establish existing conditions for populations of waterbirds that forage primarily in widely dispersed irrigated agricultural fields in the Imperial Valley, rather than at the Salton Sea (great blue herons; great, snowy and cattle egrets; white-faced ibises; and sandhill cranes), but use communal roost sites by the Salton Sea. 


\section{Purpose and Justification}

Several species of birds (great blue herons; great, snowy, and cattle egrets; white-faced ibises; and sandhill cranes) that use the Salton Sea area forage widely over the entire watershed. This behavior complicates surveys for these species because of the level of effort needed to get adequate coverage. Because these species roost communally, however, counts at roost sites are an effective and efficient method for documenting the relative abundance of those species that range widely during the day. For this monitoring plan, bird counts at roost sites would be used to identify trends in the abundance of these birds within the Salton Sea area. Survey results are intended to quantify bird use (abundance) at roosting areas around the Salton Sea and document changes over time. Resource managers can use this information to draw preliminary conclusions that guide restoration activities and the design or management of created habitats. While the information collected by this monitoring activity alone possibly will not be sufficient to determine if observed changes in abundance, species composition, and distribution are statistically significant, the surveys could serve to assess the validity of assumptions in the conceptual models and guide development of focused investigations to further refine the conceptual models as needed.

\section{Locations}

Monitoring locations will be based on past survey results and local information on roosting site locations. Additional sites can be added if other roosting areas are discovered or changes in roosting behavior are identified. Roosting locations could be located in the shoreline, halophytic scrub, agricultural land, or created freshwater impoundment geographic strata.

\section{Period and Frequency}

Bird counts at roost sites initially are proposed to be performed for at least 3 years, after which the need and scale of monitoring can be re-evaluated. Each year, roost site counts could be performed from January through March and August through December. Specific seasons and dates of surveys will be established in consultation with the Focused Technical Group and other experts. Multiple counts at each roost site will be performed during the monitoring period for an assessment of variability. The monitoring of roosting birds at the Salton Sea can be closely coordinated and integrated with the ongoing work performed by others in the Salton Sea area.

\section{Protocol and Data Collection}

With minor modifications to the protocols and data collected, these surveys can be based on the 1999 Comprehensive Shoreline Survey at the Salton Sea (Shuford and others, 2000) and designed to provide an estimate of the number of waterbirds roosting in areas proximate to the Salton Sea on the days of the survey. Counts need to be taken simultaneously at three to six sites on each survey. Observers (at least two at each site) are to be in place about 1.5 hours before dusk to count the number of great blue herons, egrets, white-faced ibises, and sandhill cranes that arrive at, or depart from, a site before nightfall. Counts will be terminated when birds stop arriving or when it becomes too dark to observe. To reduce the likelihood of double-counting birds that leave sites before dark to eventually roost at another site, the departures will be subtracted from the sum of the arrivals and those present at the onset of the count to arrive at the net total use of the roost site.

All roosting birds observed will be identified to the species level (or lowest taxon possible) and enumerated for an estimate of the number of roosting birds at individual roost sites. In addition to observations of roosting birds, habitat characteristics of the roost sites will be recorded at the time of the 
survey or determined from other sources. These characteristics of the habitat will be selected with the intent of identifying attributes that support successful roosting and that could be created or influenced through restoration actions.

\section{Data Analysis}

Observations of roosting waterbirds can be used to estimate bird numbers and species composition at the roost sites on an annual basis and to assess changes over the duration of the MAP. It is recognized that the survey results provide a "snapshot" of bird use on the day(s) of the survey and cannot provide precise quantitative estimates of bird use at roost sites around the Salton Sea. Survey results can be used in conjunction with other information to identify important ecological variables that potentially affect use of roost sites by waterbirds at the Salton Sea. This information also could contribute to establishing correlates of roosting bird distribution with other environmental attributes during the assessment phase of the MAP and to guide management activities at the Salton Sea.

\section{Quality Assurance}

As described previously, a detailed monitoring protocol will be developed prior to initiating monitoring activities in the field. This protocol will include a description of the measures that ensure the quality of the data collected and how to implement those measures. The data quality-assurance measures can include, but are not limited to, procedures for calibrating or ensuring the accuracy of any instruments (for example, GPS) employed in the field, procedures for recording and transferring electronic data, methods for ensuring proper operation of field equipment during surveys, and methods for avoiding double counting or insufficient coverage of survey areas.

\section{Data Reporting}

Data will be archived and made accessible from the CDFW's BIOS database when appropriate. Data submitted to the CDFW must be provided in a format suitable for inclusion in the BIOS database (see the "Data Management" section), including appropriate metadata. In addition, an annual progress report will summarize the data collected during that year and update prior reports in a cumulative fashion. At a minimum, the annual report will include the following:

- GIS maps showing locations of roosting sites.

- Species composition and relative abundance of roosting waterbirds at the sites.

- Environmental information collected during the bird counts at roost sites.

- Recommendations for improvement of the survey protocol, if any.

Overview of Similar Current Monitoring Activities

The USFWS currently completes annual roost surveys for sandhill cranes.

\section{Bird Surveys (Agricultural Lands)}

The following describes annual monitoring activities for establishing trend data for populations of birds that primarily use irrigated-agricultural fields in the Imperial and Coachella Valleys. 


\section{Purpose and Justification}

Because of its location relative to the Pacific Flyway, the agricultural land in the Salton Sea area supports habitat for many species of migratory and wintering birds. While most of the bird species that use the agricultural lands do so almost exclusively, some species (for example, gull-billed terns) use both the Salton Sea and nearby agricultural areas (Patten and others, 2003). Monitoring bird use in agricultural areas could document trends in the use of the area by these species and provide information that helps answer key questions related to avian resources in the Salton Sea area. Survey results can provide a general picture of bird use of agricultural fields near the Salton Sea and allow researchers and resource managers to draw preliminary conclusions about changes over time that can guide restoration activities and the design and management of created habitats.

Locations

Surveys could be performed in the agricultural areas of the Imperial Valley that compose the agricultural-land stratum.

Period and Frequency

Bird surveys in agricultural lands initially are proposed to be performed for at least 3 years, after which the need and scale of monitoring can be re-evaluated.

\section{Protocol and Data Collection}

The protocol for monitoring birds on agricultural lands can follow the procedures currently used by the IID (appendix 1) that use a combination of transects and point counts at established stations in the valley. Other protocols can be developed for monitoring bird use of agricultural lands, as needed, to collect the desired information.

\section{Data Analysis}

The information collected during monitoring activities in agricultural lands will be used to establish existing conditions in relation to bird use of these areas and document changes in the number of birds observed and species composition over time. Survey results, in conjunction with environmental data collected at the time of the surveys and during other monitoring activities, will be used to identify important environmental attributes that affect bird use of agricultural areas and to help answer key questions related to bird use in agricultural fields.

\section{Quality Assurance}

As described previously, a detailed monitoring protocol will be developed prior to initiating monitoring activities in the field. This protocol will include a description of the measures that ensure the quality of the data collected and how to implement those measures. The data quality-assurance measures can include, but are not limited to, procedures for calibrating or ensuring the accuracy of any instruments (for example, GPS) employed in the field, procedures for recording and transferring electronic data, methods for ensuring proper operation of field equipment during surveys, and methods for avoiding double counting or insufficient coverage of survey areas.

\section{Data Reporting}

Data will be archived and made accessible from the CDFW's BIOS database when appropriate. Data submitted to the CDFW must be provided in a format suitable for inclusion in the BIOS database 
(see the "Data Management" section), including appropriate metadata. In addition, an annual progress report will summarize the data collected during that year and update prior reports in a cumulative fashion. At a minimum, the annual report will include the following:

- GIS maps showing locations of monitoring sites.

- Species composition and relative abundance of birds observed at the sites.

- Environmental information collected during the surveys.

- Recommendations for improvement of the survey protocol, if any.

Overview of Similar Current Monitoring Activities

The IID currently performs transect surveys and point counts for birds in the agricultural areas within its water-service area in the Imperial Valley to comply with existing environmental permits. These surveys are intended as an inventory of existing conditions for the bird species that use agricultural areas. The IID expects to use the information obtained from this monitoring to develop a long-term monitoring program for selected bird species associated with agricultural fields that will document trends in bird use and define the environmental conditions correlated to bird use. IID also expects to use this information to better manage the agricultural areas for selected bird species, if necessary.

\section{Bird Surveys (Halophytic Scrub)}

The following sections describe the annual monitoring activities needed to meet the monitoring objectives for bird species that primarily use areas of halophytic scrub around the Salton Sea. Results of this monitoring activity, in combination with other bird surveys, could be used to establish and characterize the current use of the Salton Sea by birds. Data collected from this activity (and other elements of the MAP) can be used to identify trends in use of the Salton Sea by bird species and the environmental attributes and conditions that influence their use.

\section{Purpose and Justification}

Halophytic vegetation, primarily tamarisk and other halophytic shrubs (for example, arrowweed), which grows in areas around the margin of the Salton Sea where adequate soil moisture is present, provides habitat for numerous bird species (Mendelsohn and others, 2006). The halophytic vegetation associated with the margin of the Salton Sea could change as a result of anticipated reductions in the water-surface elevation of the Salton Sea. Halophytic scrub could encroach onto exposed playa as the lake recedes or it could remain at its current extent. Changes in vegetation can influence the use of these areas by birds. In addition to natural colonization of this vegetation on exposed playa, measures associated with the Salton Sea restoration to address the potential for dust emissions from exposed Salton Sea playa could include the active planting of halophytic vegetation. Monitoring bird use of these areas is expected to provide the basis for identifying trends in bird use and to help address key questions related to avian resources at the Salton Sea. This information also could be useful in the design and management of potential air-quality improvement actions at the Salton Sea. 


\section{Locations}

Prior to performing surveys, the extent of halophytic vegetation in the geographic stratum (within $1 \mathrm{~km}$ of the Salton Sea) needs to be delineated and quantified. Monitoring could be performed at selected locations where halophytic scrub is established or has recently colonized within $1 \mathrm{~km}$ of the shoreline. Monitoring locations will be selected randomly from the patches of halophytic scrub within this area. To the extent that suitable survey locations are available, survey locations will be distributed around the margin of the Salton Sea to ensure that survey locations are representative of all halophytic vegetation types around the margin of the lake.

\section{Period and Frequency}

Bird surveys initially are proposed to be performed for at least 3 years, after which the need and scale of monitoring can be re-evaluated. Each year, surveys could be performed during the late winter, spring migration, breeding season, early fall, and early winter. Specific seasons and dates of surveys will be established in consultation with the Focused Technical Group and other experts. Multiple observations at selected shoreline locations will be performed during each season for an assessment of variability.

The monitoring in halophytic scrub will focus primarily on landbirds, although shorebirds could be encountered in the survey where halophytic vegetation grows near the Salton Sea shoreline. With the exception of some overlap with shorebirds that could be detected in the Salton Sea shoreline surveys, the information collected in halophytic scrub would be independent of the other bird monitoring activities in the Salton Sea area. Opportunities could exist to coordinate this monitoring activity with monitoring activities performed by the IID.

\section{Protocol and Data Collection}

Birds using areas dominated by halophytic scrub around the margin of the Salton Sea can be surveyed by using methods similar to those identified by Ralph and others (1993). The methods for characterizing habitat at survey locations will be developed and included in the protocol.

\section{Data Analysis}

The information collected through monitoring bird populations is expected to be used to document trends in the number of birds observed and species composition over time. Survey results, in conjunction with environmental data collected at the time of the surveys or by other monitoring activities, can be used to identify important environmental attributes that affect bird use of halophytic scrub and help answer key questions related to bird use in halophytic scrub.

\section{Quality Assurance}

As described previously, a detailed monitoring protocol will be developed prior to initiating monitoring activities in the field. This protocol will include a description of the measures that ensure the quality of the data collected and how to implement those measures. The data quality-assurance measures can include, but are not limited to, procedures for calibrating or ensuring the accuracy of any instruments (for example, GPS) employed in the field, procedures for recording and transferring electronic data, methods for ensuring proper operation of field equipment during surveys, and methods for avoiding double counting or insufficient coverage of survey areas. 


\section{Data Reporting}

Data will be archived and made accessible from the CDFW's BIOS database when appropriate. Data submitted to the CDFW must be provided in a format suitable for inclusion in the BIOS database (see the "Data Management" section), including appropriate metadata. In addition, an annual progress report will summarize the data collected during that year and update prior reports in a cumulative fashion. At a minimum, the annual report will include the following:

- GIS maps showing survey locations.

- An estimate of the annual and seasonal abundance of birds using halophytic scrub at the Salton Sea, including confidence intervals around all estimates, where appropriate.

- Species composition and relative abundance of birds observed.

- Environmental information collected during the surveys.

- Recommendations for improvement of the survey protocol, if any.

Overview of Similar Current Monitoring Activities

IID is currently performing bird surveys throughout their water-service area, including areas dominated by halophytic vegetation (tamarisk scrub), as part of the conditions of existing environmental permits. These initial surveys are expected to continue for a period of three years, with continued monitoring scheduled over the long term.

\section{Dead and Sick Bird Counts}

The following describes monitoring activities to collect data on the incidence, magnitude, and vectors of disease outbreaks in birds at the Salton Sea.

Purpose and Justification

Several avian diseases have been identified at the Salton Sea that occasionally result in substantial bird losses (Friend, 2002). While these diseases will persist at the Salton Sea in the future, the occurrence, scale, and magnitude of these diseases could be influenced by management actions. Collection and examination of dead and sick birds can provide information on trends in the magnitude of avian diseases at the Salton Sea and the conditions that lead to, sustain, or exacerbate outbreaks of disease.

Locations

Disease outbreaks can occur anywhere in the Salton Sea area. Monitoring is to be performed in response to disease outbreaks at the locations where the outbreak occurred. The majority of sick and dead bird have been observed near the river mouths and other "fresh" water areas around the lake (K. Riesz, CDFW, pers. comm. 2011) There can be disease outbreaks in any of the geographic strata; however, detection of these outbreaks is most likely in the visible open water and shoreline areas of the Salton Sea and created impoundments. 


\section{Period and Frequency}

Surveys are proposed be performed monthly to detect disease outbreaks. Once detected, the frequency of sampling can be increased. Because disease outbreaks are episodic, collection and examination of dead and dying birds could be performed whenever the USFWS determines that significant mortality has been detected.

\section{Protocol and Data Collection}

Dead and dying birds will be collected and enumerated. It is anticipated that airboats can be used to access areas where sick and dying birds can be found. Other collection methods can be used as appropriate. Samples need to be retained from diseased birds and analyzed to determine the causal agent and mode of transmission.

\section{Data Analysis}

Counts of dead and dying birds can be used to describe and to assess changes in the incidence of avian disease outbreaks (timing, magnitude, and species affected) on an annual basis over the duration of the MAP. This information can be used in conjunction with other information, such as information on other physical and environmental variables collected by other monitoring teams, to identify important ecological variables that affect the incidence of avian disease outbreaks at the Salton Sea. This information could be used to guide management activities at the Salton Sea, including the development of focused investigations and possibly the construction and management of created habitats.

\section{Quality Assurance}

As described previously, a detailed monitoring protocol will be developed prior to initiating monitoring activities in the field. This protocol will include a description of the measures that ensure the quality of the data collected and how to implement those measures. The data quality-assurance measures can include, but are not limited to, procedures for calibrating or ensuring the accuracy of any instruments (for example, GPS) employed in the field, procedures for recording and transferring electronic data, methods for ensuring proper operation of field equipment during surveys, and methods for avoiding double counting or insufficient coverage of survey areas.

\section{Data Reporting}

When disease outbreaks occur and dead and dying birds are collected, an annual report will summarize the data collected during that year and update prior reports in a cumulative fashion. At a minimum, the annual report will include the following:

- GIS maps showing areas of concentrated mortality.

- Number of birds affected.

- Species composition of birds affected.

- Causative agents and mode of transmission, when known.

- Environmental information collected during the surveys.

- Recommendations for improvement of the survey protocol, if any. 
Overview of Similar Current Monitoring Activities

The CDFW and USFWS currently track bird mortality events at the Salton Sea. This monitoring activity will be coordinated with these ongoing activities.

\section{Fish Resources Monitoring Activities}

The eutrophic and hypersaline nature of the Salton Sea sustains a highly productive environment that currently supports large populations of salt-tolerant tilapia, which, in turn make the Salton Sea attractive to birds that rely on fish as a food source. Because the Salton Sea is a terminal water body, in the absence of any restoration actions, salinity will continue to increase, which eventually will lead to conditions that no longer support fish, and fish-eating birds (for example, pelicans, terns, cormorants and herons) will be forced to forage elsewhere. In addition to their role as a forage base for birds, historically, fish in the Salton Sea have supported a recreational fishery.

Except for desert pupfish, the Salton Sea and nearby water bodies are dominated by introduced fish species, particularly tilapia (Riedel and others, 2002; California Department of Fish and Game, 2008). Developing an understanding of the trends in fish populations at the Salton Sea (and adjacent water bodies) and the environmental conditions that influence their abundance and distribution would provide insights into the ecological role that fish play at the Salton Sea and how fish populations might be affected by restoration actions.

Desert pupfish, an endangered species, inhabit the Salton Sea, shoreline pools, and many of the drains and creeks that discharge directly to the Salton Sea. Desert pupfish are also found in a number of constructed habitats around the lake. Monitoring desert pupfish populations allows for trends in their status to be evaluated as conditions change at the Salton Sea. This information can be used to inform future management and to promote long-term conservation goals. The specific objectives of the fishresources monitoring activities and their intended purpose are presented in table 4.

Surveys of fish resources need to cover both the open water and shoreline strata and account for vertical stratification within the Salton Sea. Fish-resources monitoring activities can be coordinated with other biological monitoring or water quality and sediment monitoring activities to promote efficiency and allow for integration of survey results. Results of the fish-resource monitoring element can be combined with results of other monitoring activities (for example, other biological, hydrologic, geographic, and water quality monitoring) during the assessment portion of the MAP to provide further insight into the ecology of fish resources at the lake and their importance to bird use at the Salton Sea. Because these activities need to be coordinated with the ongoing monitoring activities performed by others (for example, the CDFW, USFWS, and USGS) in the Salton Sea area, a web-based integration tool, such as the master sample-tracking and management system developed by Pacific Northwest Aquatic Management Program (PNAMP) could be considered during development of the Implementation Plan. This approach will help with the integration of the various components, will help facilitate the experimental design, and will help facilitate data integration and sharing.

\section{Fish Surveys (Salton Sea)}

The following sections describe the annual monitoring activities needed to meet the monitoring objectives for fish species that inhabit the Salton Sea and its tributaries. Monitoring results can be used to establish and characterize the current status of fish populations in the Salton Sea and its tributaries. Data collected from this and other elements of the MAP) could be used to identify trends in fish populations at the Salton Sea and the environmental attributes and conditions that influence them. 


\section{Purpose and Justification}

Fish play an important role in the Salton Sea aquatic ecosystem and provide a forage base for several fish-eating bird species. Anticipated changes in water quality of the Salton Sea will continue to affect fish populations and the bird species that rely on them. Monitoring fish populations at the Salton Sea can establish the current status of fish populations, establish a framework to allow monitoring of trends in fish populations (catch, percent occupancy, abundance) over time, and address key questions related to fish and fish-eating birds at the Salton Sea. The monitoring information also can support informed decision making regarding potential restoration or species' conservation actions at the Salton Sea. Survey results can provide a general indication of the health of fish populations at the Salton Sea and provide resource managers and researchers information to guide restoration activities and the design or management of created habitats. The survey results could contribute to the development of focused investigations, where they are needed to refine conceptual models.

Locations

To obtain data on the patterns of distribution and relative catch rates of various fish species in the Salton Sea, sampling could be performed at a number of locations along the shore, near river mouths, and in deep water in both the north and south basins of the lake. To the extent appropriate, sampling locations will be similar in depths and substrates to locations sampled by the CDFW during their quarterly gillnet sampling (California Department of Fish and Game, 2008). Sampling locations will be randomly selected from both the Salton Sea open-water and shoreline strata to ensure that survey data are representative of the entire Salton Sea. The shoreline stratum could be further subdivided to ensure adequate sampling in shoreline areas thought to support higher fish populations (for example, delta areas at the mouths of the Alamo and New Rivers).

\section{Period and Frequency}

Fish surveys at the Salton Sea initially are proposed to be performed for at least 3 years, after which the need and scale of monitoring can be re-evaluated. Site-specific surveys are to be performed monthly throughout the year. One complete survey of all locations is to be performed during each month. Fish monitoring activities are to be coordinated with invertebrate, water-quality, and sediment monitoring activities, whenever possible.

\section{Protocol and Data Collection}

It is anticipated that the primary sampling gear will be multi-panel gillnets of varying mesh size (California Department of Fish and Game, 2008); however, various sampling methods are anticipated, and specific protocols will be developed for these methods, as appropriate for the different depths and habitat types, to improve the likelihood that all sizes and species of fish that inhabit the Salton Sea are sampled. Care will be taken to minimize the lethal incidental catch of non-target species, such as desert pupfish and diving birds. Shallow areas (shoreline pools, or river mouths) cannot be sampled effectively by using gillnets; other sampling methods will be identified or developed for use in these areas.

Information on sampling effort (set/soak time, area sampled, or volume trawled) will be recorded for each gear type.

All captured fish will be identified to the species level and enumerated. An adequate number of fish of each species will be measured (length) and weighed. Scales, bony structures, or whole fish will be collected to assess the age distribution of each species' populations. When possible, the breeding condition of individuals will be determined and recorded. Individual fish can be frozen for subsequent 
analysis of breeding condition and stomach contents. Coincident with all sampling, other water-quality and physical-habitat attribute measurements will be measured, including depth, water temperature, dissolved oxygen, and electrical conductivity (salinity), to help determine the role of environmental attributes on species composition, fish distribution, and health. Sample locations will be georeferenced by using GPS coordinates.

\section{Data Analysis}

Catch-per-unit-effort (CPUE) calculated from the catch and effort data, or other indices of abundance of fish at the Salton Sea, will be used to assess changes in fish populations on a seasonal and annual basis. It is recognized that the survey results only provide a snapshot of fish catches at the time of the survey and cannot be used to provide precise quantitative estimates of fish abundance at the Salton Sea; however, the collection of monthly samples allows for an assessment of seasonal variability and a comparison of changes in CPUE over time.

Fish survey results can be used in conjunction with other information, such as other environmental variables measured during the surveys and by other monitoring teams, to evaluate important ecological variables that could affect fish populations at the Salton Sea. They also can be used to inform management activities at the Salton Sea, including the development of focused investigations. Information collected through the fish monitoring could provide insights into the design and management of saline water bodies to conserve fish and wildlife at the Salton Sea.

\section{Quality Assurance}

A detailed monitoring protocol will be developed prior to initiating monitoring activities. This protocol will include a description of the sampling procedures, dates, times, locations, a description of measures that ensure the quality of the data collected and how to implement those measures. The data quality-assurance measures will include, but are not limited to, procedures for calibrating or ensuring the accuracy of any instruments (for example, water quality meters) deployed in the field, procedures for recording and transferring electronic data, and methods for ensuring proper operation of equipment during surveys.

\section{Data Reporting}

Data will be archived and made accessible from the CDFW's BIOS database when appropriate. Data submitted to the CDFW must be provided in a format suitable for inclusion in the BIOS database (see the "Data Management" section), including appropriate metadata. In addition, an annual progress report will summarize the data collected during that year and update prior reports. At a minimum, the annual report will include the following:

- GIS maps depicting the location of each sample location.

- Annual and seasonal catches (CPUE) of fish at the Salton Sea for each gear type.

- Species composition and relative abundance of fish captured in the Salton Sea for each gear type.

- Size/age distribution of fish captured in the Salton Sea for each gear type.

- Breeding condition of fish captured in the Salton Sea for each gear type. 
- Environmental information collected during the surveys.

- Recommendations for improvement of the survey protocol, if any.

Overview of Similar Current Monitoring Activities

The CDFW performed seasonal gillnet sampling at the Salton Sea until boat launching access deteriorated in 2008 (California Department of Fish and Game, 2008). Once fish monitoring activities resume, the prior sampling protocols will be reviewed and revised for integration into the monitoring protocol.

\section{Desert Pupfish Surveys}

The following sections describe the annual monitoring activities needed to meet the monitoring objectives for desert pupfish and other fish species that inhabit the Salton Sea and tributaries to the Salton Sea. Results of this monitoring activity will be used to describe the current distribution of desert pupfish in tributaries to the Salton Sea and use of the Salton Sea shoreline by this species. Data collected from this activity (and other elements of the MAP) will be used to identify changes in pupfish distribution at the Salton Sea and the environmental attributes and conditions influencing the use of drains, washes, and other tributaries to the lake; shoreline pools; and open water at the lake.

\section{Purpose and Justification}

The desert pupfish is both federally and state-listed as an endangered species. The status and health of desert-pupfish populations in and near the Salton Sea are influenced by changes in water quality and water-surface elevation at the Salton Sea, interactions with nonnative fish species, and, possibly, by habitat creation associated with restoration. Monitoring information that provides insights into desert pupfish distribution (and potentially movement) and abundance relative to other fish species over time could help address key questions related to desert pupfish at the Salton Sea. Understanding how desert pupfish currently use the Salton Sea and its tributaries and how they respond to changing conditions also could help resource managers with future decisions regarding desert pupfish management.

\section{Locations}

To obtain data on the patterns of distribution and abundance of desert pupfish in the various habitats in the Salton Sea area, sampling needs to be performed in a stratified random, or other appropriate, sampling design in drains that discharge directly to the Salton Sea, in washes, along the margin of the Salton Sea (including nearshore areas, river mouths, and shoreline pools), and in created freshwater and saline impoundments. Where possible, sampling locations will include those currently sampled by the CDFW and USGS as part of their desert pupfish monitoring (for example, Keeney, 2009; Saiki and others, 2008, 2010).

\section{Period and Frequency}

Desert pupfish surveys at the Salton Sea initially are proposed to be performed for at least 3 years, after which the need and scale of monitoring can be re-evaluated. Desert-pupfish surveys will be performed monthly. Existing data will be evaluated to provide guidance on the development of a statistically valid sampling strategy. Because a large number of pupfish habitats exist, it is not possible to sample all locations in all years. Review of existing data can allow for the establishment of a 
minimum threshold for locations to be sampled. A survey of all selected locations are to be performed during each month. Opportunities could exist to coordinate this monitoring activity with ongoing monitoring through identifying common objectives and coordinating the work to improve efficiency.

\section{Protocol and Data Collection}

The primary sampling gear will likely be baited minnow traps, similar to what is currently used by the CDFW (DFG, 2009). However, it is recognized that this strategy could require revision to increase sampling precision, and this has been identified as a high priority focused study need. Minnow traps will be supplemented with other gear types (for example, dipnets, seines), as appropriate for the different depths and habitat types, to increase the likelihood of capture of desert pupfish and other species in areas of low abundance and where capture by using minnow traps possibly would not be effective. All captured fish will be identified to the species level and enumerated. An adequate number of individuals of each species will be measured (length) and weighed. Scales, bony structures, or whole fish will be collected to assess the age distribution of each species' population. When possible, the breeding condition of individuals will be determined and recorded. Individuals of species other than desert pupfish can be frozen for later analysis of length, breeding condition, and stomach contents. Marking of desert pupfish and other fish species can be performed as a coordinated focused investigation to assess movement between and among habitats. Coincident with all sampling, other water-quality and physical habitat attributes will be measured, including depth, water temperature, dissolved oxygen, and electrical conductivity (salinity), to help determine the role of environmental attributes on species composition, fish distribution, and health. Sample locations will be georeferenced by GPS coordinates.

\section{Data Analysis}

Catch data can be used to describe the distribution and relative abundance of desert pupfish in the various habitats of the Salton Sea and to evaluate changes in the occupancy of potential desert pupfish habitat in the Salton Sea. By using the catch data collected during sampling, the distribution of pupfish in the various habitats sampled will be evaluated monthly and annually to assess changes in the distribution of desert pupfish over the duration of the MAP. Wherever and whenever possible, information on trap efficiency will be developed and used to estimate population sizes in selected habitats. Mark-recapture methods can be used to evaluate any movement of desert pupfish between areas or habitats. This information will be used in conjunction with other data to evaluate ecological variables in relation to desert pupfish abundance and distribution at the Salton Sea. This information can guide focused investigations and management activities at the Salton Sea, including the construction and management of created habitats.

The ongoing desert pupfish surveys performed by the CDFW and USGS improve our understanding of the factors influencing desert pupfish movement and survival. The CDFW's surveys are intended to monitor the status of desert pupfish in the Salton Sea area. The USGS is examining the potential for selenium in agricultural drainage to affect desert pupfish populations (Besser and others, 2012). To the extent possible, results of these monitoring activities need to be integrated during the assessment phase of the MAP.

\section{Quality Assurance}

A detailed monitoring protocol would be developed prior to initiating monitoring activities. This protocol will include a description of the sampling procedures, dates, times, locations, description of measures that ensure the quality of the data collected and how to implement those measures. The data 
quality-assurance measures will include, but are not limited to, procedures for calibrating or ensuring the accuracy of any instruments (for example, water-quality meters) deployed in the field, procedures for recording and transferring electronic data, and methods for ensuring proper operation of equipment during surveys.

\section{Data Reporting}

Data will be archived and made accessible from the CDFW's BIOS database when appropriate. Data submitted to the CDFW must be provided in a format suitable for inclusion in the BIOS database (see the "Data Management" section), including appropriate metadata. In addition, an annual progress report will summarize the data collected during that year and update prior reports. At a minimum, the annual report will include the following:

- GIS maps of sampling locations and locations where desert pupfish are captured.

- Annual and seasonal catches (number of fish captures) of desert pupfish and other fish species in various habitats at the Salton Sea.

- The distribution of desert of pupfish in various habitats at the Salton Sea.

- Species composition and relative abundance of fish captured during desert pupfish surveys.

- Size/age distribution of pupfish and other fish species captured during the surveys.

- Breeding condition of fish captured during the surveys.

- Environmental information collected during the surveys.

- Recommendations for improvement of the survey protocol, if any.

\section{Overview of Similar Current Monitoring Activities}

The CDFW currently performs pupfish sampling on an annual, bi-annual, monthly, and more frequent basis (depending on habitat) at selected drains at the north and south ends of the lake, at marina and other shoreline areas, at washes near Hot Mineral Springs, at San Felipe Creek, at Salt Creek (lower and upper), and at refuge (artificial) habitats (Keeney, 2009). The USGS also has performed pupfish sampling in drains, ponds, creeks, and created saline impoundments in the Imperial Valley and Coachella Valley (Martin and Saiki 2005, 2009; Saiki 1990; Saiki and others 2008, 2011).

\section{Plankton and Macroinvertebrate Resources Monitoring Activities}

Planktonic and larger macroinvertebrate organisms form the foundation of the aquatic food chain and monitoring these organisms can provide insights into the health of the ecosystem and the factors that influence their abundance in the Salton Sea and created habitats. The information acquired through this monitoring plan can help identify potential correlates between changes observed at higher trophic levels and changes in the invertebrate fauna. The specific objectives of the plankton and macroinvertebrate monitoring activities and their intended purpose are presented in table 5.

Results of the phytoplankton, zooplankton, and macroinvertebrate surveys within the Salton Sea, created saline impoundments, and created freshwater impoundments will be integrated on an annual and 
seasonal basis during the assessment phase of the MAP to develop a more complete understanding of invertebrate resources at the Salton Sea. For sampling efficiency, it is desirable to collect phytoplankton, zooplankton, and benthic macroinvertebrate samples at the same locations. In addition, coordinating the benthic macroinvertebrate sampling with water-quality and sediment monitoring activities (see the "Water and Sediment Quality" section of this monitoring plan) produces co-located information that can contribute to an understanding of how selenium and other contaminants cycle within ecosystem.

\section{Water-Column Phytoplankton Surveys (Salton Sea)}

The following sections describe the annual monitoring activities needed to meet the monitoring objectives for phytoplankton species in the water column at the Salton Sea. Results of this monitoring activity will be used to characterize the current phytoplankton populations in the Salton Sea. Data collected from this activity (and other elements of the MAP) will be used to identify trends in phytoplankton density and species composition at the Salton Sea as well as the environmental attributes and conditions that influence them.

Purpose and Justification

The phytoplankton community is a fundamental component and an indicator of the health of the Salton Sea ecosystem. Because of its response to water quality and trophic position, monitoring phytoplankton can provide early insights into changes in the Salton Sea ecosystem and responses at other trophic levels. The phytoplankton surveys are designed to document the density and species composition of phytoplankton in the Salton Sea.

Locations

Phytoplankton sampling will be performed at selected locations in the open-water stratum and the shoreline stratum identified for the Salton Sea. Sample locations in the open-water stratum will be selected along the deep-water axis of the Salton Sea, which generally lies at the midpoint of the lake parallel to the north-south axis, including areas in the north and south basins, where depths exceed 9 meters. Three of these sampling locations will be consistent with locations previously sampled by Reclamation and Tiffany and others (2007) and will correspond to the sampling stations established for water-quality sampling under the MAP. The locations in the shoreline stratum will be located in depths of 2 to 8 meters and be randomly distributed around the perimeter of the lake.

Period and Frequency

Phytoplankton surveys at the Salton Sea are proposed to be performed for at least 3 years during the initial phase of the MAP. One complete phytoplankton survey is to be performed each month at each location. The activities under this monitoring element will be coordinated with the other monitoring elements, including those activities performed on state, federal, tribal, and private lands.

\section{Protocol and Data Collection}

Samples in the open-water stratum will be collected by using a 3-meter integrated sampler (tube, or other appropriate device) to collect samples from the 0-3 meter, 3-6 meter, and 6-9 meter depth ranges, as described in Tiffany and others (2007). Samples will be mixed in the field, and samples for analysis will be taken from the composite. The shoreline stratum will be sampled by using a Van Dorn (or similar) water-collection device of known volume to collect phytoplankton from the middle of the vertical water column. It is assumed that the shallow shoreline areas are adequately mixed so that this 
technique could provide a good estimate of average conditions throughout the vertical water column. Replicate samples will be collected and analyzed for species composition, and all organisms will be identified to the lowest taxonomic level practical. Some species that are difficult to identify could be analyzed as a group (for example, small flagellates). Samples will be preserved for later analysis. Separate samples for analysis of chlorophyll levels will be taken from the composite, filtered in the field, and preserved by freezing of the intact filter for later analysis. An adequate number of replicates will be collected for an estimate of sample variability.

Coincident with sample collection, other water-quality and physical habitat attributes will be measured, including water depth, water temperature, dissolved oxygen, and electrical conductivity (salinity). Sample locations will be identified in the field by using GPS.

\section{Data Analysis}

Phytoplankton density (number of organisms per unit volume), ascertained from samples of known volume on a monthly and annual basis, will be used to describe the relative abundance and composition of phytoplankton at the Salton Sea and to assess changes over time. Replicate samples will be used to assess sample variability, which allows for a statistical comparison of changes in density over time.

Results of this monitoring activity, in combination with other investigations, will be used to establish and characterize the existing conditions in relation to the density and species composition of phytoplankton in the Salton Sea and, in the assessment phase of the MAP, to identify changes in the density and species composition of phytoplankton in the Salton Sea.

These data also can be used in conjunction with other information, such as information on other environmental variables collected during the surveys by other monitoring teams, to identify important ecological variables (water temperature, dissolved oxygen, and salinity) that could affect phytoplankton populations at the Salton Sea. This information can be used to document changes over time and to guide future activities at the Salton Sea, including focused investigations and possibly the construction and management of created saline impoundments.

\section{Quality Assurance}

As described previously, a detailed monitoring protocol will be developed prior to initiating monitoring activities in the field. This protocol will include a description of the measures that ensure the quality of the data collected and how to implement those measures. For any laboratory tests, a qualityassurance project plan will be prepared prior to sampling. The data quality-assurance measures can include, but are not limited to, procedures for calibrating or ensuring the accuracy of any instruments

(for example, GPS) employed in the field, procedures for recording and transferring electronic data, and methods for ensuring proper operation of field equipment during surveys.

\section{Data Reporting}

Data will be archived and made accessible from the CDFW's BIOS database when appropriate. Data submitted to the CDFW must be provided in a format suitable for inclusion in the BIOS database (see the "Data Management" section), including appropriate metadata. In addition, an annual progress report will summarize the data collected during that year and update prior reports in a cumulative fashion. At a minimum, the annual report will include the following: 
- GIS maps of the sampling locations.

- Annual and monthly density (as an indicator of abundance) of phytoplankton captured at the Salton Sea.

- Annual and monthly species composition of phytoplankton captured in the Salton Sea.

- Annual and monthly chlorophyll concentrations observed at the Salton Sea.

- Sample variability based on the replicate samples.

- Environmental attributes measured during the surveys.

- Recommendations for improvement of the survey protocol, if any.

Overview of Similar Current Monitoring Activities

No similar monitoring activities are currently in progress at the Salton Sea, although researchers at San Diego State University have collected and analyzed phytoplankton samples from the Salton Sea previously (Tiffany and others, 2007).

\section{Water-Column Phytoplankton Surveys (Created Saline Impoundments)}

The following describes annual monitoring activities to establish existing conditions for phytoplankton in created saline impoundments. Data collected from this activity (and other elements of the MAP) can be used to identify trends in phytoplankton density and species composition in created saline impoundments as well as the environmental attributes and conditions that influence them.

Purpose and Justification

As in the Salton Sea, the phytoplankton community will play an important role in the created shallow habitat ecosystems and will serve as an indicator of the health of the aquatic community. Because of their rapid response to changes in water quality and their trophic position, monitoring phytoplankton provides early insights into changes in the shallow-habitat ecosystem and responses at other trophic levels. The phytoplankton surveys are designed to document the density and species composition of phytoplankton in created saline impoundments.

Locations

Phytoplankton sampling will be performed at several (three to five) locations within each created saline pond selected for monitoring. Sampling locations within these ponds will be determined during development of the sample design

\section{Period and Frequency}

Phytoplankton surveys in created saline impoundments initially are proposed to be performed for at least 3 years, after which the need and scale of monitoring can be re-evaluated. One complete phytoplankton survey will be performed each month at each location. The activities under this monitoring element will be coordinated with the other monitoring elements, including those activities performed on state, federal, tribal, and private lands. 


\section{Protocol and Data Collection}

Because the existing ponds are generally shallow (less than 2 meters), samples will be collected by using a Van Dorn (or similar water collection device of known volume) sampler to collect phytoplankton from the middle of the water column. It is assumed that the shallow ponds are adequately mixed so that this technique can provide a good estimate of average conditions over the entire vertical water column. If deeper areas are encountered or ponds of greater depth are constructed in the future and added to the sampling stratum, an integrated sampler (tube, or other appropriate device) could be used to collect samples. Replicate samples will be collected and analyzed for species composition, and all organisms will be identified to the lowest taxonomic level practical. Some species that are difficult to identify could be analyzed as a group (for example, small flagellates). Samples will be preserved for later analysis. Separate sub-samples for analysis of chlorophyll levels will be taken from the same sample, filtered in the field, and preserved for analysis. An adequate number of replicates will be collected for an estimate of sample variability.

Coincident with sample collection, other water-quality and physical habitat attributes will be measured, including water depth, water temperature, dissolved oxygen, and electrical conductivity (salinity). Sample locations will be identified in the field by using GPS.

\section{Data Analysis}

Phytoplankton density (number of organisms per unit volume), determined from samples of known volume on a quarterly and annual basis, will be used to describe the relative abundance and composition of phytoplankton in the created saline impoundments and to assess changes in abundance and composition over the duration of the MAP. Replicate samples will be used to assess sample variability, which allows for a statistical comparison of changes in density over time.

Results of this monitoring activity, in combination with other studies, will be used to determine and characterize the existing conditions in relation to the density and species composition of phytoplankton in created saline impoundments and to document trends in abundance and composition of phytoplankton in the those areas. They also can be used in conjunction with other information, such as information on other environmental variables collected during the surveys by other monitoring teams, to identify important ecological variables that affect phytoplankton abundance and diversity in these shallow water bodies. This information can be used to document changes over time and to guide management activities for these created habitats.

\section{Quality Assurance}

As described previously, a detailed monitoring protocol will be developed prior to initiating monitoring activities in the field. This protocol will include a description of the measures that ensure the quality of the data collected and how to implement those measures. For any laboratory tests, a qualityassurance project plan will be prepared prior to sampling. The data quality-assurance measures can include, but are not limited to, procedures for calibrating or ensuring the accuracy of any instruments (for example, GPS) employed in the field, procedures for recording and transferring electronic data, and methods for ensuring proper operation of field equipment during surveys.

\section{Data Reporting}

Data will be archived and made accessible from the CDFW's BIOS database when appropriate. Data submitted to the CDFW must be provided in a format suitable for inclusion in the BIOS database (see the "Data Management" section), including appropriate metadata. In addition, an annual progress 
report will summarize the data collected during that year and update prior reports in a cumulative fashion. At a minimum, the annual report will include the following:

- GIS maps of the sampling locations.

- Annual and quarterly density (as an indicator of abundance) of phytoplankton collected in the created saline impoundments.

- Annual and quarterly species composition of phytoplankton collected in the created saline impoundments.

- Annual and quarterly chlorophyll concentrations observed in the created saline impoundments.

- Sample variability based on the replicate samples.

- Environmental attributes measured during the surveys.

- Recommendations for improvement of the survey protocol, if any.

Overview of Similar Current Monitoring Activities

No similar monitoring activities are currently underway at the Salton Sea.

Water-Column Phytoplankton Surveys (Created Freshwater Impoundments)

The following describes annual monitoring activities to establish existing conditions for phytoplankton in created freshwater impoundments. Data collected from this activity (and other elements of the MAP) will be used to identify trends in phytoplankton density and species composition in created freshwater impoundments as well as the environmental attributes and conditions that influence them.

Purpose and Justification

Created freshwater impoundments are under consideration as a component of aquatic systems created under ecosystem restoration at the Salton Sea. As in the Salton Sea, the phytoplankton community in these created shallow habitat ecosystems could play an important role and serve as an indicator of the health of the aquatic community. Because of their rapid response to changes in water quality and their trophic position, monitoring phytoplankton could provide early insights into changes in the shallow habitat ecosystem as well as responses at other trophic levels. The phytoplankton surveys are designed to document the density and species composition of phytoplankton in created freshwater impoundments.

Locations

To obtain data on the density and species composition of phytoplankton, several freshwater impoundments adjacent to the Salton Sea will be randomly selected for annual monitoring. Survey locations will be chosen to represent a range of conditions (for example, depth, substrate).

Impoundments included in the surveys could be located on adjacent state, federal, tribal, or private lands. 
Phytoplankton sampling will be performed at several (three to five) locations within each created freshwater impoundment selected for monitoring. Sampling locations within these ponds will be determined during development of the sample design.

\section{Period and Frequency}

Phytoplankton surveys in created freshwater impoundments initially are proposed to be performed for at least 3 years, after which the need and scale of monitoring can be re-evaluated. Sampling will be performed monthly throughout the year. One complete phytoplankton survey will be performed each month at each location. The activities under this monitoring element will be coordinated with the other monitoring elements, including those activities performed on state, federal, tribal, and private lands.

\section{Protocol and Data Collection}

Because the existing ponds are generally shallow (less than 2 meters), samples will be collected by using a Van Dorn sampler (or similar water collection device of known volume) to collect phytoplankton from the middle of the water column. It is assumed that the shallow ponds are adequately mixed so that this technique can provide a good estimate of average conditions throughout the water column. If deeper areas are encountered or ponds of greater depth are constructed in the future and added to the sampling stratum, an integrated sampler (tube, or other appropriate device) could be used to collect samples. Replicate samples will be collected and analyzed for species composition, and all organisms will be identified to the lowest taxonomic level practical. Some species that are difficult to identify could be analyzed as a group (for example, small flagellates). Samples will be preserved for later analysis. Separate sub-samples for analysis of chlorophyll levels will be taken from the same sample, filtered in the field, and preserved for analysis. An adequate number of replicates will be collected for an estimate of sample variability.

Coincident with sample collection, other water-quality and physical habitat attributes will be measured, including water depth, water temperature, dissolved oxygen, and electrical conductivity (salinity). Sample locations will be identified in the field by using GPS.

\section{Data Analysis}

Phytoplankton density (number of organisms per unit volume), determined from samples of known volume on a quarterly and annual basis, will be used to describe the relative abundance and composition of phytoplankton in the created freshwater impoundments and to assess changes in abundance and composition over the duration of the MAP. Replicate samples of phytoplankton will be used to assess sample variability, which allows for a statistical comparison of changes in density over time.

Results of this monitoring activity, in combination with other studies, will be used to establish and characterize the existing conditions in relation to the density and species composition of phytoplankton in created freshwater impoundments and to document trends in abundance and composition of phytoplankton in the those areas. These data also can be used in conjunction with other information, such as information on other environmental variables collected during the surveys by other monitoring teams, to identify important ecological variables that affect phytoplankton abundance and diversity in these shallow water bodies. This information will be used to document changes over time and to guide management activities for these created habitats. 


\section{Quality Assurance}

As described previously, a detailed monitoring protocol will be developed prior to initiating monitoring activities in the field. This protocol will include a description of the measures that ensure the quality of the data collected and how to implement those measures. For any laboratory tests, a qualityassurance project plan will be prepared prior to sampling. The data quality-assurance measures can include, but are not limited to, procedures for calibrating or ensuring the accuracy of any instruments (for example, GPS) employed in the field, procedures for recording and transferring electronic data, and methods for ensuring proper operation of field equipment during surveys.

\section{Data Reporting}

Data will be archived and made accessible from the CDFW's BIOS database when appropriate. Data submitted to the CDFW must be provided in a format suitable for inclusion in the BIOS database (see the "Data Management" section), including appropriate metadata. In addition, an annual progress report will summarize the data collected during that year and update prior reports in a cumulative fashion. At a minimum, the annual report will include the following:

- GIS maps of the sampling locations.

- Annual and quarterly density (as an indicator of abundance) of phytoplankton collected in the created freshwater impoundments.

- Annual and quarterly species composition of phytoplankton collected in the created freshwater impoundments.

- Annual and quarterly chlorophyll concentrations measured in the created freshwater impoundments.

- Sample variability based on the replicate samples.

- Environmental attributes collected during the surveys.

- Recommendations for improvement of the survey protocol, if any.

Overview of Similar Current Monitoring Activities

No similar monitoring activities are currently underway at the Salton Sea.

\section{Water-Column Zooplankton Surveys (Salton Sea)}

The following sections describe the annual monitoring activities needed to meet the monitoring objectives for zooplankton species within the water column at the Salton Sea. Results of this monitoring activity will be used to characterize the current zooplankton populations in the Salton Sea. Data collected from this activity (and other elements of the MAP) will be used to identify trends in zooplankton density and species composition at the Salton Sea as well as the environmental attributes and conditions that influence them. 


\section{Purpose and Justification}

Zooplankton represent an important component of the aquatic ecosystem in the Salton Sea. Zooplankton influence the abundance and diversity of phytoplankton populations and provide a necessary link in the food chain of fish and other wildlife at the Salton Sea. Monitoring zooplankton at the Salton Sea is needed to describe zooplankton populations, identify changes in zooplankton populations, and to collect information that would help address key questions related to zooplankton and other aquatic resources. The zooplankton surveys are designed to document the relative abundance and species composition of zooplankton in the Salton Sea.

\section{Locations}

Zooplankton sampling will be performed at selected locations in the open-water stratum and the shoreline stratum identified for the Salton Sea. The locations in the open-water stratum will be selected along the deep-water axis of the Salton Sea, which generally lies at the midpoint of the lake parallel to the north-south axis, including areas in the north and south basins, where depths exceed 9 meters. Three of these sampling locations will be consistent with locations sampled previously by Reclamation and Tiffany and others $(2002,2007)$ and will correspond to the sampling stations established for water quality sampling under the MAP. The locations in the shoreline stratum will be located in depths of 2 to 8 meters and be randomly distributed around the perimeter of the lake.

\section{Period and Frequency}

Zooplankton surveys at the Salton Sea initially are proposed to be performed for at least 3 years, after which the need and scale of monitoring can be re-evaluated. One complete zooplankton survey will be performed each month at each location. The activities under this monitoring element will be coordinated with the other monitoring elements, including those activities performed on state, federal, tribal, and private lands.

\section{Protocol and Data Collection}

In the open-water stratum, zooplankton can be sampled by performing vertical tows from near the bottom to the surface of the lake by using a standard, $80-\mu \mathrm{m}$ mesh zooplankton net or with a Schindler-Patalas trap (or other suitable collection device with a known volume) at specific intervals in the water column (for example Tiffany and others, 2002). A flow-meter will be installed in the net to estimate the sampled volume. In the shallower shoreline stratum, samples can be collected by using a suitable collection device with a known volume (for example, Schindler-Patalas trap) to collect zooplankton from the middle of the water column; however, an alternative sampling device, capable of sampling the entire water column, could be necessary. Replicate samples will be collected and analyzed at each station, and all organisms identified to the lowest taxonomic level practical. Some species that are difficult to identify could be analyzed as a group. Samples will be preserved for later analysis. An adequate number of replicates will be collected for an estimate of sample variability.

Coincident with sample collection, other water-quality and physical habitat attributes will be measured, including water depth, water temperature, dissolved oxygen, and electrical conductivity (salinity). Sample locations will be identified in the field by using GPS.

\section{Data Analysis}

Zooplankton density (number of organisms per unit volume) calculated from the number captured and estimated sample volume on a monthly and annual basis will be used to describe the 
relative abundance and species composition of zooplankton at the Salton Sea and to assess changes over time. Replicate samples will be used to assess sample variability, which allows for a statistical comparison of changes in density over time.

Results of this monitoring activity, in combination with other investigations, will be used to determine and characterize the existing conditions in relation to the density and species composition of zooplankton in the Salton Sea and, in the assessment phase of the MAP, to identify changes in the density and species composition of zooplankton in the Salton Sea.

They also can be used in conjunction with other information, such as other environmental variables collected during the surveys and by other monitoring teams, to identify important ecological variables (water temperature, dissolved oxygen, and salinity) that affect zooplankton populations at the Salton Sea. This information can be used to document changes over time and to guide activities at the Salton Sea, including focused investigations and possibly the construction and management of created saline impoundments.

\section{Quality Assurance}

As described previously, a detailed monitoring protocol will be developed prior to initiating monitoring activities in the field. This protocol will include a description of the measures that ensure the quality of the data collected and how to implement those measures. The data quality-assurance measures can include, but are not limited to, procedures for calibrating or ensuring the accuracy of any instruments (for example, GPS) employed in the field, procedures for recording and transferring electronic data, and methods for ensuring proper operation of field equipment during surveys.

\section{Data Reporting}

Data will be archived and made accessible from the CDFW's BIOS database when appropriate. Data submitted to the CDFW must be provided in a format suitable for inclusion in the BIOS database (see the "Data Management" section), including appropriate metadata. In addition, an annual progress report will summarize the data collected during that year and update prior reports in a cumulative fashion. At a minimum, the annual report will include the following:

- GIS maps of the sampling locations.

- Annual and monthly density (as an indicator of relative abundance) of zooplankton at the Salton Sea.

- Species composition of zooplankton captured at the Salton Sea on a monthly and annual basis.

- Sample variability based on the replicate samples.

- Environmental attributes collected during the surveys.

- Recommendations for improvement of the survey protocol, if any.

\section{Overview of Similar Current Monitoring Activities}

No similar monitoring activities are currently underway at the Salton Sea, although researchers at San Diego State University have collected and analyzed zooplankton samples from the Salton Sea previously (for example, Tiffany and others, 2002). 


\section{Water-Column Zooplankton Surveys (Created Saline Impoundments)}

The following describes monitoring activities to establish existing conditions for zooplankton in the created saline impoundments. Data collected from this activity (and other elements of the MAP) will be used to identify trends in zooplankton density and species composition in created saline impoundments as well as the environmental attributes and conditions that influence them.

\section{Purpose and Justification}

Zooplankton are an important component of the aquatic ecosystem in created habitats and critical to the success of these water bodies for supporting fish and birds. Monitoring in these created habitats is necessary to describe zooplankton populations and identify changes in the invertebrate community that can influence fish or birds using the created habitats for foraging. The zooplankton surveys are designed to document the density and species composition of zooplankton in the created saline impoundments.

Locations

Zooplankton sampling will be performed at several locations (three to five) within each created saline pond selected for monitoring. Sampling locations within these ponds will be determined during development of the sample design and will be the same locations sampled for phytoplankton.

\section{Period and Frequency}

Zooplankton surveys in the created saline impoundments initially are proposed to be performed for at least 3 years, after which the need and scale of monitoring can be re-evaluated. One complete zooplankton survey will be performed each month at each location. The activities under this monitoring element will be coordinated with the other monitoring elements, including those activities performed on state, federal, tribal, and private lands.

\section{Protocol and Data Collection}

Because the existing ponds are generally shallow (less than 2 meters), samples can be collected by using a Schindler-Patalas trap (or other suitable collection device with a known volume) to collect zooplankton from the middle of the water column (see Tiffany and others, 2002). It is assumed that the shallow ponds are adequately mixed so that this technique can provide a good estimate of average conditions throughout the water column. If ponds of greater depth are constructed in the future, an alternative sampling device capable of sampling the entire water column could be necessary. Replicate samples will be analyzed for species composition, and all organisms will be identified to the lowest taxonomic level practical. Some species that are difficult to identify could be analyzed as a group. An adequate number of replicates will be collected for an estimate of sample variability. Samples will be preserved for later analysis.

Coincident with sample collection, other water-quality and physical habitat attributes will be measured, including water depth, water temperature, dissolved oxygen, and electrical conductivity (salinity). Sample locations will be identified in the field by using GPS.

\section{Data Analysis}

Zooplankton density (number of organisms per unit volume), determined from the samples of known volume on a monthly and annual basis, will be used to describe the relative abundance and composition of zooplankton in the created saline impoundments and to assess changes over time. 
Replicate samples will be used to assess sample variability, which allows for a statistical comparison of changes in abundance over time.

Results of this monitoring activity, in combination with other investigations, will be used to establish and characterize the existing conditions in relation to the density and species composition of zooplankton and, in the assessment phase of the MAP, to identify changes in the density and species composition of zooplankton in the created saline impoundments.

They also can be used in conjunction with other information, such as other environmental variables measured during the surveys and by other monitoring teams, to identify important ecological variables that affect zooplankton abundance and composition in these shallow water bodies. This information will be used to document changes over time and to guide management activities, including focused investigations and possibly the construction and management of created saline impoundments.

\section{Quality Assurance}

As described previously, a detailed monitoring protocol will be developed prior to initiating monitoring activities in the field. This protocol will include a description of the measures that ensure the quality of the data collected and how to implement those measures. The data quality-assurance measures can include, but are not limited to, procedures for calibrating or ensuring the accuracy of any instruments (for example, GPS) employed in the field, procedures for recording and transferring electronic data, and methods for ensuring proper operation of field equipment during surveys.

\section{Data Reporting}

Data will be archived and made accessible from the CDFW's BIOS database when appropriate. Data submitted to the CDFW must be provided in a format suitable for inclusion in the BIOS database (see the "Data Management" section), including appropriate metadata. In addition, an annual progress report will summarize the data collected during that year and update prior reports in a cumulative fashion. At a minimum, the annual report will include the following:

- GIS maps of the sampling locations.

- Annual and quarterly density (as an indicator of relative abundance) of zooplankton captured in the created saline impoundments.

- Species composition of zooplankton captured in the created saline impoundments on a quarterly and annual basis.

- Sample variability based on the replicate samples.

- Environmental attributes collected during the surveys.

- Recommendations for improvement of the survey protocol, if any.

Overview of Similar Current Monitoring Activities

No similar monitoring activities are currently underway at the Salton Sea. 


\section{Water-Column Zooplankton Surveys (Created Freshwater Impoundments)}

The following describes monitoring activities to establish existing conditions for zooplankton in the created freshwater impoundments. Data collected from this activity (and other elements of the MAP) will be used to identify trends in zooplankton density and species composition in created freshwater impoundments as well as the environmental attributes and conditions that influence them.

\section{Purpose and Justification}

Created freshwater impoundments are under consideration as a component of aquatic systems created under ecosystem restoration at the Salton Sea. Zooplankton are an important component of the aquatic ecosystem in created habitats and critical to the success of these water bodies in supporting fish and birds. Monitoring in these created habitats is necessary to describe zooplankton populations and identify changes in the invertebrate community that could influence fish or birds using the created habitats for foraging. The zooplankton surveys are designed to document the density and species composition of zooplankton in the created freshwater impoundments.

\section{Locations}

To obtain data on the density and species composition of zooplankton, several freshwater impoundments adjacent to the Salton Sea will be randomly selected for annual monitoring. Survey locations will be chosen to represent a range of conditions (for example, depth, salinity). Impoundments included in the surveys could be located on adjacent state, federal, tribal, or private lands.

Zooplankton sampling will be performed at several locations (three to five) within each created freshwater impoundment selected for monitoring. Sampling locations within these ponds will be determined during development of the sample design and will be to the same as the locations sampled for phytoplankton.

\section{Period and Frequency}

Zooplankton surveys in the created freshwater impoundments initially are proposed to be performed for at least 3 years, after which the need and scale of monitoring can be re-evaluated. One complete zooplankton survey will be performed each month at each location. The activities under this monitoring element will be coordinated with the other monitoring elements, including those activities performed on state, federal, tribal, and private lands.

\section{Protocol and Data Collection}

Because the existing ponds are generally shallow (less than 2 meters), samples will be collected by using a Schindler-Patalas trap (or other suitable collection device with a known volume) to collect zooplankton from the middle of the water column (see Tiffany and others, 2002). It is assumed that the shallow ponds are adequately mixed so that this technique can provide a good estimate of average conditions throughout the water column. If this assumption is determined to be incorrect, or if ponds of greater depth are constructed in the future and added to the sampling stratum, an alternative sampling device capable of sampling the entire water column could be necessary. Replicate samples will be analyzed for species composition, and all organisms will be identified to the lowest taxonomic level practical. Some species that are difficult to identify could be analyzed as a group. An adequate number of replicates will be collected to allow for an estimate of sample variability. Samples will be preserved for later analysis. 
Coincident with sample collection, other water quality and physical habitat attributes will be measured, including water depth, water temperature, dissolved oxygen, and electrical conductivity. Sample locations will be identified in the field by using GPS.

\section{Data Analysis}

Zooplankton density (number of organisms per unit volume), determined from the samples of known volume on a monthly and annual basis, will be used to describe the relative abundance and composition of zooplankton in the created freshwater impoundments and to assess changes over time. Replicate samples will be used to assess sample variability, which allows for a statistical comparison of changes in abundance over time.

Results of this monitoring activity, in combination with other investigations, will be used to establish and characterize the existing conditions in relation to the density and species composition of zooplankton and, in the assessment phase of the MAP, to identify changes in the density and species composition of zooplankton in the created freshwater impoundments.

They also will be used in conjunction with other information, such as other environmental variables measured during the surveys and by other monitoring teams, to identify important ecological variables that affect zooplankton abundance and composition in these shallow water bodies. This information will be used to document changes over time and to guide management activities, including focused investigations and possibly the construction and management of created freshwater impoundments.

\section{Quality Assurance}

As described previously, a detailed monitoring protocol will be developed prior to initiating monitoring activities in the field. This protocol will include a description of the measures that ensure the quality of the data collected and how to implement those measures. The data quality-assurance measures can include, but are not limited to, procedures for calibrating or ensuring the accuracy of any instruments (for example, GPS) employed in the field, procedures for recording and transferring electronic data, and methods for ensuring proper operation of field equipment during surveys.

\section{Data Reporting}

Data will be archived and made accessible from the CDFW's BIOS database when appropriate. Data submitted to the CDFW must be provided in a format suitable for inclusion in the BIOS database (see the "Data Management" section), including appropriate metadata. In addition, an annual progress report will summarize the data collected during that year and update prior reports in a cumulative fashion. At a minimum, the annual report will include the following:

- GIS maps of the sampling locations.

- Annual and quarterly density (as an indicator of relative abundance) of zooplankton captured in the created freshwater impoundments.

- Species composition of zooplankton captured in the created freshwater impoundments on a quarterly and annual basis.

- Sample variability based on the replicate samples. 
- Environmental attributes collected during the surveys.

- Recommendations for improvement of the survey protocol, if any.

Overview of Similar Current Monitoring Activities

No similar monitoring activities are currently underway at the Salton Sea.

\section{Water-Column Macroinvertebrate Surveys (Salton Sea)}

The following sections describe the annual monitoring activities needed to meet the monitoring objectives for macroinvertebrate species within the water column at the Salton Sea. Results of this monitoring activity will be used to characterize the current macroinvertebrate populations in the Salton Sea. Data collected from this activity (and other elements of the MAP) will be used to identify trends in macroinvertebrate density and species composition at the Salton Sea as well as the environmental attributes and conditions that influence them.

\section{Purpose and Justification}

Water-column macroinvertebrates (for example, pileworms and corixids) represent an important component of the Salton Sea ecosystem and serve as food items for birds and fish. The abundance and diversity of macroinvertebrates are expected to change with changes in water quality. The monitoring of water-column macroinvertebrates at the Salton Sea is necessary to describe macroinvertebrate populations, identify changes in water-column macroinvertebrate populations, and to collect information that could help address key questions related to macroinvertebrates and other aquatic resources. This monitoring also will provide insights into the environmental conditions that support macroinvertebrates at the Salton Sea and contribute information that could be useful in designing and managing created habitats that support macroinvertebrate populations.

\section{Locations}

Water-column macroinvertebrate sampling will be performed at selected locations in the openwater and shoreline strata identified for the Salton Sea. The locations in the open-water stratum will be selected along the deep-water axis of the Salton Sea, including areas in the north and south basins, where depths exceed 9 meters. Three of these sampling locations will be consistent with locations sampled previously by Reclamation and Tiffany and others $(2002,2007)$ and correspond to the sampling stations established for water-quality sampling under the MAP. The locations in the shoreline stratum will be located in depths of 2 to 8 meters and be randomly distributed around the perimeter of the lake.

\section{Period and Frequency}

Water-column macroinvertebrate surveys at the Salton Sea initially are proposed to be performed for at least 3 years, after which the need and scale of monitoring can be re-evaluated. One complete macroinvertebrate survey will be performed each month at each location. Results of the watercolumn macroinvertebrate surveys within the Salton Sea will be integrated with results from other resource surveys (that is, phytoplankton and zooplankton) on an annual and seasonal basis during the assessment phase of the MAP to develop a more complete understanding of invertebrate resources at the Salton Sea. For efficiency in sampling, it is desirable to collect macroinvertebrate samples in the same locations as phytoplankton and zooplankton samples. The activities under this monitoring element will 
be coordinated with the other monitoring elements, including those activities performed on state, federal, tribal, and private lands.

\section{Protocol and Data Collection}

Samples will be collected by using a large mesh (approximately 1 millimeter), large diameter (0.5 meter) plankton net towed through the water column (or other suitable collection device, for example, Schindler-Patalas trap). Deep-water samples will be collected by using vertical tows from the bottom to the surface of the lake or by trapping at specific water depths, and shallow-water samples will be collected by using horizontal, mid-water tows or by trapping along a transect. A flow-meter will be installed in the net to estimate the sampled volume. Replicate samples will be collected and analyzed at each station, and all organisms will be identified to the lowest taxonomic level practical. Some species that are difficult to identify could be analyzed as a group. An adequate number of replicates will be collected to allow for an estimate of sample variability. Samples will be preserved for later analysis.

Coincident with sample collection, other water-quality and physical habitat attributes will be measured, including water depth, water temperature, dissolved oxygen, and electrical conductivity (salinity). Sample locations will be identified in the field by using GPS.

\section{Data Analysis}

Water-column macroinvertebrate density (number of organisms per unit volume), determined from the number captured and estimated sample volume on a monthly and annual basis, will be used to describe the relative abundance and species composition of water-column macroinvertebrates at the Salton Sea and to assess changes over time. Replicate samples will be used to assess sample variability, which allows for a statistical comparison of changes in abundance over time.

Results of this monitoring activity, in combination with other investigations, will be used to establish and characterize the existing conditions in relation to the density and species composition of water-column macroinvertebrates in the Salton Sea and, in the assessment phase of the MAP, to identify changes in the density and species composition of these organisms in the Salton Sea.

They also will be used in conjunction with other information, such as other environmental variables measured during the surveys and by other monitoring teams, to identify important ecological variables that affect water-column macroinvertebrate populations at the Salton Sea. This information will be used to document changes over time and to guide management activities at the Salton Sea, including focused investigations and possibly the construction and management of created saline impoundments.

\section{Quality Assurance}

As described previously, a detailed monitoring protocol will be developed prior to initiating monitoring activities in the field. This protocol will include a description of the measures that ensure the quality of the data collected and how to implement those measures. The data quality-assurance measures can include, but are not limited to, procedures for calibrating or ensuring the accuracy of any instruments (for example, GPS) employed in the field, procedures for recording and transferring electronic data, and methods for ensuring proper operation of field equipment during surveys.

\section{Data Reporting}

Data will be archived and made accessible from the CDFW's BIOS database when appropriate. Data submitted to the CDFW must be provided in a format suitable for inclusion in the BIOS database (see the "Data Management" section), including appropriate metadata. In addition, an annual progress 
report will summarize the data collected during that year and update prior reports in a cumulative fashion. At a minimum, the annual report is to include the following:

- GIS maps of the sampling locations.

- Annual and quarterly density (as an indicator of relative abundance) of water-column macroinvertebrates.

- Species composition of water-column macroinvertebrates captured in the Salton Sea on a monthly and annual basis.

- Sample variability based on the replicate samples.

- Environmental attributes collected during the surveys.

- Recommendations for improvement of the survey protocol, if any.

Overview of Similar Current Monitoring Activities

No similar monitoring activities are currently underway at the Salton Sea, although researchers at San Diego State University have collected and analyzed macroinvertebrate samples from the Salton Sea previously (for example, Detwiler and others, 2002).

Water-Column Macroinvertebrate Surveys (Created Saline Impoundments)

The following describes monitoring activities to establish existing conditions for water column macroinvertebrates in the created saline impoundments. Data collected from this activity (and other elements of the MAP) will be used to identify trends in macroinvertebrate density and species composition in created saline impoundments as well as the environmental attributes and conditions that influence them.

Purpose and Justification

Water-column macroinvertebrates (for example, pileworms and corixids) represent an important component of the aquatic ecosystem in created habitats, and they are critical to the success of these water bodies in the support of fish and birds. The monitoring of water-column macroinvertebrates in the created saline impoundments is necessary to describe macroinvertebrate populations and changes in the invertebrate community that can affect the success of fish or birds foraging in the created saline impoundments. The data collected through monitoring also will contribute to answering key questions related to macroinvertebrates and other aquatic resources. The information gained through this monitoring activity is directly applicable to the design and management of habitats created to support birds and fish.

\section{Locations}

Water-column macroinvertebrate sampling will be performed at several locations (three to five) within each created saline pond selected for monitoring. Sampling locations within these ponds will be determined during development of the sample design and will be similar to the locations sampled for plankton. 


\section{Period and Frequency}

Macroinvertebrate surveys in the created saline impoundments initially are proposed to be performed for at least 3 years, after which the need and scale of monitoring can be re-evaluated. One complete survey will be performed each month at each location. Results of the water-column macroinvertebrate surveys within the created saline impoundments will be integrated with results from other resource surveys (that is, phytoplankton, zooplankton) on an annual and seasonal basis during the assessment phase of the MAP to develop a more complete understanding of invertebrate resources at the impoundments and at the Salton Sea. For efficiency in sampling, it is desirable to collect macroinvertebrate samples in the same locations as phytoplankton and zooplankton samples. The activities under this monitoring element will be coordinated with the other monitoring elements, including those activities performed on state, federal, tribal, and private lands.

\section{Protocol and Data Collection}

A 1-mm mesh, framed net (or other suitable collection device) will be used to collect macroinvertebrates from throughout the water column and along vegetation edges with approximately equal levels of effort per sampling area and habitat type. Samples from each location will be mixed in the field, and the composite will be analyzed to describe macroinvertebrate density and species composition within the sampled saline pond. Replicate samples will be collected from each pond and analyzed for species composition, and all organisms will be identified to the lowest taxonomic level practical. Some species that are difficult to identify could be analyzed as a group. An adequate number of replicates will be collected for an estimate of sample variability. Samples will be preserved for later analysis.

Coincident with sample collection, other water-quality and physical habitat attributes will be measured, including water depth, water temperature, dissolved oxygen, and electrical conductivity (salinity). Sample locations will be identified by using GPS.

\section{Data Analysis}

The density (number of organisms per unit volume) of water-column macroinvertebrates will be roughly estimated from the number captured. Replicate samples will be used to assess sample variability, which allows for a statistical comparison of changes in density over time.

Results of this monitoring activity, in combination with other investigations, will be used to establish and characterize the existing conditions in relation to the density and species composition of water-column macroinvertebrates and, in the assessment phase of the MAP, to identify changes in the density and species composition of these organisms in the created saline impoundments.

They also will be used in conjunction with other information, such as other environmental variables measured during the surveys and by other monitoring teams, to identify important ecological variables that affect macroinvertebrate abundance and composition in the created saline impoundments.

\section{Quality Assurance}

As described previously, a detailed monitoring protocol will be developed prior to initiating monitoring activities in the field. This protocol will include a description of the measures that ensure the quality of the data collected and how to implement those measures. The data quality-assurance measures can include, but are not limited to, procedures for calibrating or ensuring the accuracy of any instruments (for example, GPS) employed in the field, procedures for recording and transferring electronic data, and methods for ensuring proper operation of field equipment during surveys. 


\section{Data Reporting}

Data will be archived and made accessible from the CDFW's BIOS database when appropriate. Data submitted to the CDFW must be provided in a format suitable for inclusion in the BIOS database (see the "Data Management" section), including appropriate metadata. In addition, an annual progress report will summarize the data collected during that year and update prior reports in a cumulative fashion. At a minimum, the annual report is to include the following:

- GIS maps of the sampling locations.

- Annual and monthly relative abundance (number captured) of macroinvertebrates.

- Species composition of macroinvertebrates captured on a quarterly and annual basis.

- Sample variability based on the replicate samples.

- Environmental attributes collected during the surveys.

- Recommendations for improvement of the survey protocol, if any.

Overview of Similar Current Monitoring Activities

No similar monitoring activities are currently underway at the Salton Sea.

Water-Column Macroinvertebrate Surveys (Created Freshwater Impoundments)

The following describes monitoring activities to establish existing conditions for water-column macroinvertebrates in the created freshwater impoundments. Data collected from this activity (and other elements of the MAP) will be used to identify trends in macroinvertebrate density and species composition in created freshwater impoundments as well as the environmental attributes and conditions that influence them.

\section{Purpose and Justification}

Created freshwater impoundments are under consideration as a component of aquatic systems created under ecosystem restoration at the Salton Sea. Water-column macroinvertebrates (for example, corixids) could represent an important component of the aquatic ecosystem supported in created habitats and could contribute to the success of these water bodies in supporting fish and birds. The monitoring of water-column macroinvertebrates in the created freshwater impoundments is necessary to describe macroinvertebrate populations and changes in the invertebrate community that could influence the success of fish or birds foraging in the created freshwater impoundments. The data collected through monitoring also will help answer key questions related to macroinvertebrates and other aquatic resources. The information gained through this monitoring activity is directly applicable to the design and management of habitats created to support birds and fish.

\section{Locations}

To obtain data on the density and species composition of macroinvertebrates, several freshwater impoundments adjacent to the Salton Sea will be randomly selected for annual monitoring. Survey locations will be chosen to represent a range of conditions (for example, depth, substrate, and salinity). 
Impoundments included in the surveys could be located on adjacent state, federal, tribal, or private lands.

Water-column macroinvertebrate sampling will be performed at several locations (three to five) within each created saline pond selected for monitoring. Sampling locations within these ponds will be determined during development of the sample design and will be the same as the locations sampled for phytoplankton and zooplankton.

\section{Period and Frequency}

Macroinvertebrate surveys in the created freshwater impoundments initially are proposed to be performed for at least 3 years, after which the need and scale of monitoring can be re-evaluated. One complete survey will be performed each month at each location. Results of the water-column macroinvertebrate surveys within the created freshwater impoundments will be integrated with results from other resource surveys (that is, phytoplankton and, zooplankton) on an annual and seasonal basis during the assessment portion of the MAP to develop a more complete understanding of invertebrate resources at the impoundments and at the Salton Sea. For efficiency in sampling, it is desirable to collect macroinvertebrate samples in roughly the same locations as phytoplankton and zooplankton samples. The activities under this monitoring element will be coordinated with the other monitoring elements, including those activities performed on state, federal, tribal, and private lands.

\section{Protocol and Data Collection}

A 1-mm-mesh, framed net (or other suitable collection device) will be used to collect macroinvertebrates from throughout the water column and along vegetation edges with approximately equal levels of effort per sampling area and habitat type. Samples from each location will be mixed in the field, and the composite will be analyzed to describe macroinvertebrate density and species composition within the sampled freshwater pond. Replicate samples will be collected from each pond and analyzed for species composition, and all organisms will be identified to the lowest taxonomic level practical. Some species that are difficult to identify could be analyzed as a group. An adequate number of replicates will be collected for an estimate of sample variability. Samples will be preserved for later analysis.

Coincident with sample collection, other water-quality and physical habitat attributes will be collected, including water depth, water temperature, dissolved oxygen, and electrical conductivity (salinity). Sample locations will be identified by using GPS.

\section{Data Analysis}

The density (number of organisms per unit volume) of water-column macroinvertebrates will be roughly estimated from the number captured. Replicate samples will be used to assess sample variability, which allows for a statistical comparison of changes in density over time.

Results of this monitoring activity, in combination with other investigations, will be used to establish and characterize the existing conditions in relation to the density and species composition of water-column macroinvertebrates and, in the assessment phase of the MAP, to identify changes in the density and species composition of these organisms in the created freshwater impoundments.

They also will be used in conjunction with other information, such as other environmental variables measured during the surveys and by other monitoring teams, to identify important ecological variables that affect macroinvertebrate abundance and composition in the created freshwater impoundments. 


\section{Quality Assurance}

As described previously, a detailed monitoring protocol will be developed prior to initiating monitoring activities in the field. This protocol will include a description of the measures that ensure the quality of the data collected and how to implement those measures. The data quality-assurance measures can include, but are not limited to, procedures for calibrating or ensuring the accuracy of any instruments (for example, GPS) employed in the field, procedures for recording and transferring electronic data, and methods for ensuring proper operation of field equipment during surveys.

\section{Data Reporting}

Data will be archived and made accessible from the CDFW's BIOS database when appropriate. Data submitted to the CDFW must be provided in a format suitable for inclusion in the BIOS database (see the "Data Management" section), including appropriate metadata. In addition, an annual progress report will summarize the data collected during that year and update prior reports in a cumulative fashion. At a minimum, the annual report is to include the following:

- GIS maps of the sampling locations.

- Annual and monthly relative abundance (number captured) of macroinvertebrates.

- Species composition of macroinvertebrates captured on a quarterly and annual basis.

- Sample variability based on the replicate samples.

- Environmental attributes collected during the surveys.

- Recommendations for improvement of the survey protocol, if any.

Overview of Similar Current Monitoring Activities

No similar monitoring activities are currently underway at the Salton Sea.

\section{Benthic Macroinvertebrate Surveys (Salton Sea)}

The following sections describe the annual activities needed to meet the monitoring objectives for macroinvertebrate species in the benthos at the Salton Sea. Results of this monitoring activity will be used to characterize the current macroinvertebrate populations in the Salton Sea. Data collected from this activity (and other elements of the MAP) will be used to identify trends in benthic macroinvertebrate density and species composition at the Salton Sea as well as the environmental attributes and conditions that influence them.

\section{Purpose and Justification}

Currently, the Salton Sea benthic macroinvertebrate community is dominated by pileworms, which are a key food source for fish and birds using the Salton Sea. Water quality in the Salton Sea is changing, and the specific conditions that support pileworms and other benthic macroinvertebrates likely will not persist. Monitoring of benthic invertebrates is needed to describe benthic macroinvertebrate populations, identify changes in their abundance, and to collect information that will help answer key questions related to invertebrates other aquatic resources. These data also could be 
useful for explaining changes detected in fish and bird use, as well as for designing and managing created habitats that support benthic invertebrates.

Locations

Benthic macroinvertebrate sampling will be performed at selected locations in the open-water and shoreline strata identified for the Salton Sea. The locations in the open-water stratum will be selected along the deep water axis of the Salton Sea, including areas in the north and south basins, where depths exceed 9 meters. Samples can be collected along transects (Detwiler and others, 2002), or at discrete sampling locations similar to locations sampled previously by Reclamation and Tiffany and others $(2002,2007)$, and will correspond to the sampling stations established for water-quality sampling under the MAP. The locations in the shoreline stratum will be located in depths of 2 to 8 meters and be randomly distributed around the perimeter of the lake. Sampling locations will be co-located with sediment sampling at all sites. In addition, hard substrates, such as rocks, submerged structures, construction materials, and imported fill (for example concrete riprap), will also be sampled at locations such as the marina jetties (Detwiler and others, 2002). Barnacle shell substrates within the shoreline stratum will also be sampled.

\section{Period and Frequency}

Benthic macroinvertebrate surveys at the Salton Sea initially are proposed to be performed for at least 3 years, after which the need and scale of monitoring can be re-evaluated. One complete survey will be performed each month at each location. Results of the benthic macroinvertebrate surveys within the Salton Sea will be integrated with results from other resource surveys (that is, phytoplankton, zooplankton) on an annual and seasonal basis during the assessment portion of the MAP to develop a more complete understanding of invertebrate resources at the Salton Sea. For efficiency in sampling, it is desirable to collect benthic macroinvertebrate samples in roughly the same locations as water-column macroinvertebrate samples. In addition, the benthic macroinvertebrate sampling will be coordinated with water-quality and sediment monitoring activities (see the "Water and Sediment Quality" section of this monitoring plan) to produce co-located information that will help clarify how selenium and other contaminants cycle within ecosystem.

\section{Protocol and Data Collection}

Samples taken from soft substrates will be collected by using a mini-Ponar or Ekman dredge (or other suitable collection device) to sample sediment and associated pileworms as well as other organisms from the top 10 to 20 centimeters of sediment over a known surface area. Samples will be sieved in the field, and samples will be collected for analysis from the $0.5-\mathrm{mm}$ mesh sieve. Hard substrates will be sampled with a scraper (see Detwiler and others, 2002) or other suitable collection device. Samples from barnacle shell substrates will be collected by using a suitable coring device. Replicate samples will be collected and analyzed for species composition, and all organisms will be identified to the lowest taxonomic level practical. Some species that are difficult to identify could be analyzed as a group. An adequate number of replicates will be collected for an estimate of sample variability. Samples will be preserved for later analysis.

Coincident with benthic macroinvertebrate sample collection, other water-quality and physical habitat attributes will be measured, including water depth, water temperature, dissolved oxygen, and electrical conductivity (salinity). Sample locations will be identified in the field by using GPS. 


\section{Data Analysis}

Benthic macroinvertebrate density (number of organisms per unit volume), determined from the number captured and the sample volume on a monthly and annual basis, will be used to describe the relative abundance and species composition of benthic macroinvertebrates at the Salton Sea and to assess changes over time. Replicate samples will be used to assess sample variability, which allows for a statistical comparison of changes in abundance over time.

Results of this monitoring activity, in combination with other investigations, will be used to establish and characterize the existing conditions in relation to the density and species composition of benthic macroinvertebrates in the Salton Sea and, in the assessment phase of the MAP, to identify changes in the density and species composition of these organisms in the Salton Sea.

They also will be used in conjunction with other information, such as other environmental variables collected during the surveys and by other monitoring teams, to identify important ecological variables that affect benthic macroinvertebrate populations at the Salton Sea. This information will be used to guide management activities at the Salton Sea, including focused investigations and possibly the construction and management of created saline impoundments.

Quality Assurance

As described previously, a detailed monitoring protocol will be developed prior to initiating monitoring activities in the field. This protocol will include a description of the measures that ensure the quality of the data collected and how to implement those measures. The data quality-assurance measures can include, but are not limited to, procedures for calibrating or ensuring the accuracy of any instruments (for example, GPS) employed in the field, procedures for recording and transferring electronic data, and methods for ensuring proper operation of field equipment during surveys.

\section{Data Reporting}

Data will be archived and made accessible from the CDFW's BIOS database when appropriate. Data submitted to the CDFW must be provided in a format suitable for inclusion in the BIOS database (see the "Data Management" section), including appropriate metadata. In addition, an annual progress report will summarize the data collected during that year and update prior reports in a cumulative fashion. At a minimum, the annual report is to include the following:

- GIS maps of the sampling locations.

- Annual and monthly density (as an indicator of relative abundance) of benthic macroinvertebrates at the Salton Sea.

- Species composition of benthic macroinvertebrates captured in the Salton Sea on an annual and quarterly basis.

- Sample variability based on the replicate samples.

- Environmental attributes collected during the surveys.

- Recommendations for improvement of the survey protocol, if any. 


\section{Overview of Similar Current Monitoring Activities}

No similar monitoring activities are currently underway at the Salton Sea, although researchers at San Diego State University have collected and analyzed benthic macroinvertebrate samples from the Salton Sea previously (for example, Detwiler and others, 2002).

\section{Benthic Macroinvertebrate Surveys (Created Saline Impoundments)}

The following describes monitoring activities to establish existing conditions for benthic macroinvertebrates in the created saline impoundments. Data collected from this activity (and other elements of the MAP) will be used to identify trends in benthic macroinvertebrate density and species composition in created saline impoundments as well as the environmental attributes and conditions that influence them.

\section{Purpose and Justification}

As in the Salton Sea, populations of benthic macroinvertebrates will contribute to the ability of created habitats to successfully support diverse fish and bird communities. Monitoring benthic invertebrate populations in created habitats is necessary to describe benthic macroinvertebrate populations, to identify changes in their abundance, and to collect information that helps address key questions related to other aquatic resources. The information gained through this monitoring activity is directly applicable to the design and management of habitats created to support birds and fish.

\section{Locations}

Benthic macroinvertebrate sampling will be performed at several locations (three to five) within each created saline pond. Locations selected for monitoring of benthic macroinvertebrates will be colocated with other biological monitoring sites (for example, phytoplankton). In addition, proposed sampling locations will be co-located with sediment sampling sites.

\section{Period and Frequency}

Benthic macroinvertebrate surveys in the created saline impoundment initially are proposed to be performed for at least 3 years, after which the need and scale of monitoring can be re-evaluated. One complete survey will be performed each month at each location. Results of the benthic macroinvertebrate surveys within the created saline impoundments will be integrated with results from other resource surveys (that is, phytoplankton and zooplankton) on an annual and seasonal basis during the assessment portion of the MAP to develop a more complete understanding of invertebrate resources at the impoundments and the Salton Sea. For efficiency, it is desirable to collect benthic macroinvertebrate samples in the same locations as water column macroinvertebrate samples. In addition, the benthic macroinvertebrate sampling will be coordinated with water-quality and sediment monitoring activities (see the "Water and Sediment Quality" section of this monitoring plan) to produce co-located information that could help clarify how selenium and other contaminants cycle within ecosystem.

\section{Protocol and Data Collection}

Because hard substrates and barnacle shell substrates are not anticipated to be major components in created saline impoundments, samples will be collected by using a mini-Ponar or Ekman dredge (or other suitable collection device) to sample sediment and associated pileworms as well as other organisms from the top 10 to 20 centimeters of sediment over a known surface area. Samples will be 
sieved in the field, and samples will be collected for analysis from the $0.5-\mathrm{mm}$ mesh sieve. Replicate samples will be collected and analyzed for species composition, and all organisms will be identified to the lowest taxonomic level practical. Some species that are difficult to identify could be analyzed as a group. An adequate number of replicates will be collected to allow for an estimate of sample variability. Samples will be preserved for later analysis.

Coincident with sample collection, other water-quality and physical habitat attributes will be measured, including water depth, water temperature, dissolved oxygen, and electrical conductivity (salinity). Sample locations will be identified in the field by using GPS.

\section{Data Analysis}

Benthic macroinvertebrate density (number of organisms per unit volume) will be determined from the number captured and the sample volume on a monthly and annual basis and will be used to describe the relative abundance and species composition of benthic macroinvertebrates in the created saline impoundments and to assess changes over time. Replicate samples will be used to assess sample variability, which allows for a statistical comparison of changes in abundance over time.

Results of this monitoring activity, in combination with other investigations, will be used to establish and characterize the existing conditions in relation to the density and species composition of benthic macroinvertebrates and, in the assessment phase of the MAP, to identify changes in the density and species composition of these organisms in the created saline impoundments.

These results also will be used in conjunction with other information, such as other environmental variables collected during the surveys and by other monitoring teams, to identify important ecological variables that affect benthic macroinvertebrate abundance and composition in the created saline impoundments.

\section{Quality Assurance}

As described previously, a detailed monitoring protocol will be developed prior to initiating monitoring activities in the field. This protocol will include a description of the measures that ensure the quality of the data collected and how to implement those measures. The data quality-assurance measures can include, but are not limited to, procedures for calibrating or ensuring the accuracy of any instruments (for example, GPS) employed in the field, procedures for recording and transferring electronic data, and methods for ensuring proper operation of field equipment during surveys.

\section{Data Reporting}

Data will be archived and made accessible from the CDFW's BIOS database when appropriate. Data submitted to the CDFW must be provided in a format suitable for inclusion in the BIOS database (see the "Data Management" section), including appropriate metadata. In addition, an annual progress report will summarize the data collected during that year and update prior reports in a cumulative fashion. At a minimum, the annual report is to include the following:

- GIS maps of the sampling locations.

- Annual and monthly density (as an indicator of relative abundance) of benthic macroinvertebrates in each created habitat cell.

- Species composition of benthic macroinvertebrates captured in each created habitat cell. 
- Sample variability based on the replicate samples.

- Environmental attributes collected during the surveys.

- Recommendations for improvement of the survey protocol, if any.

Overview of Similar Current Monitoring Activities

No similar monitoring activities are currently underway at the Salton Sea.

Benthic Macroinvertebrate Surveys (Created Freshwater Impoundments)

The following describes monitoring activities to establish existing conditions for benthic macroinvertebrates in the created freshwater impoundments. Data collected from this activity (and other elements of the MAP) will be used to identify trends in benthic macroinvertebrate density and species composition in created freshwater impoundments as well as the environmental attributes and conditions that influence them.

Purpose and Justification

Created freshwater impoundments are under consideration as a component of aquatic systems created under ecosystem restoration at the Salton Sea. As in the Salton Sea, populations of benthic macroinvertebrates could contribute to the ability of created habitats to successfully support diverse fish and bird communities. Monitoring benthic invertebrate populations in created habitats is necessary to describe benthic macroinvertebrate populations, identify changes in their abundance, and to collect information that could help address key questions related to other aquatic resources. The information gained through this monitoring activity is directly applicable to the design and management of habitats created to support birds and fish.

Locations

To obtain data on the density and species composition of phytoplankton, several freshwater impoundments adjacent to the Salton Sea will be randomly selected for annual monitoring. Survey locations will be chosen to represent a range of conditions (for example, depth, substrate, and salinity). Impoundments included in the surveys could be located on adjacent state, federal, tribal, or private lands.

Benthic macroinvertebrate sampling will be performed at several locations (three to five) within each created freshwater impoundment. Locations selected for monitoring of benthic macroinvertebrates will be co-located with other biological monitoring sites (for example, phytoplankton). In addition, proposed sampling locations will be co-located with sediment sampling sites.

\section{Period and Frequency}

Benthic macroinvertebrate surveys in the created freshwater impoundment initially are proposed to be performed for at least 3 years, after which the need and scale of monitoring can be re-evaluated. One complete survey will be performed each month at each location. Results of the benthic macroinvertebrate surveys within the created freshwater impoundments will be integrated with results from other resource surveys (that is, phytoplankton and zooplankton) on an annual and seasonal basis during the assessment portion of the MAP to develop a more complete understanding of invertebrate resources at the impoundments and the Salton Sea. For efficiency, it is desirable to collect benthic 
macroinvertebrate samples in roughly the same locations as water-column macroinvertebrate samples. In addition, the benthic macroinvertebrate sampling will be coordinated with water-quality and sediment monitoring activities (see the "Water and Sediment Quality" section of this monitoring plan) to produce co-located information that could help clarify how selenium and other contaminants cycle within ecosystem.

\section{Protocol and Data Collection}

Because hard substrates and barnacle shell substrates are not anticipated to be found in created freshwater impoundments, samples will be collected by using a mini-Ponar or Ekman dredge (or other suitable collection device) to sample sediment and associated organisms from the top 10 to 20 centimeters of sediment over a known surface area. Samples will be sieved in the field, and samples will be collected for analysis from the $0.5-\mathrm{mm}$ mesh sieve. Replicate samples will be collected and analyzed for species composition, and all organisms will be identified to the lowest taxonomic level practical. Some species that are difficult to identify could be analyzed as a group. An adequate number of replicates will be collected for an estimate of sample variability. Samples will be preserved for later analysis.

Coincident with sample collection, other water-quality and physical habitat attributes will be measured, including water depth, water temperature, dissolved oxygen, and electrical conductivity (salinity). Sample locations will be identified in the field by using GPS.

\section{Data Analysis}

Benthic macroinvertebrate density (number of organisms per unit volume) will be determined from the number captured and the sample volume on a monthly and annual basis and will be used to describe the relative abundance and species composition of benthic macroinvertebrates in the created freshwater impoundments and to assess changes over time. Replicate samples will be used to assess sample variability, which allows for a statistical comparison of changes in abundance over time.

Results of this monitoring activity, in combination with other investigations, will be used to establish and characterize the existing conditions in relation to the density and species composition of benthic macroinvertebrates and, in the assessment phase of the MAP, to identify changes in the density and species composition of these organisms in the created freshwater impoundments.

These results also will be used in conjunction with other information, such as other environmental variables collected during the surveys and by other monitoring teams, to identify important ecological variables that affect benthic macroinvertebrate abundance and composition in the created freshwater impoundments.

\section{Quality Assurance}

As described previously, a detailed monitoring protocol will be developed prior to initiating monitoring activities in the field. This protocol will include a description of the measures that ensure the quality of the data collected and how to implement those measures. The data quality-assurance measures can include, but are not limited to, procedures for calibrating or ensuring the accuracy of any instruments (for example, GPS) employed in the field, procedures for recording and transferring electronic data, and methods for ensuring proper operation of field equipment during surveys.

\section{Data Reporting}

Data will be archived and made accessible from the CDFW's BIOS database when appropriate. Data submitted to the CDFW must be provided in a format suitable for inclusion in the BIOS database 
(see the "Data Management" section), including appropriate metadata. In addition, an annual progress report will summarize the data collected during that year and update prior reports in a cumulative fashion. At a minimum, the annual report is to include the following:

- GIS maps of the sampling locations.

- Annual and monthly density (as an indicator of relative abundance) of benthic macroinvertebrates in each created freshwater impoundment.

- Species composition of benthic macroinvertebrates captured in each created freshwater impoundment.

- Sample variability based on the replicate samples.

- Environmental attributes collected during the surveys.

- Recommendations for improvement of the survey protocol, if any.

Overview of Similar Current Monitoring Activities

No similar monitoring activities are currently underway at the Salton Sea.

Vector (mosquito) Monitoring

The following describes monitoring activities needed to establish existing conditions that support mosquitoes in created impoundments. This will be complementary to any monitoring or data collection performed by local vector-control districts.

Purpose and Justification

Salton Sea restoration will result in the creation of water bodies for water conveyance, recreation, and habitat. Depending on how they are managed and water quality, these water bodies could support populations of mosquitoes, which increases the threat that they could become vectors for disease. Mosquito monitoring in created impoundments will be necessary to describe mosquito abundance and provide data necessary for vector management and control.

Locations

Mosquito larvae sampling will be performed in both created freshwater and created saline impoundment strata in association with and at the same locations as the invertebrate sampling.

Period and Frequency

Mosquito larvae surveys in the created impoundments initially are proposed to be performed for at least 3 years, after which the need and scale of monitoring can be re-evaluated. One complete survey will be performed each month at each location. The monitoring of mosquitoes will be coordinated with the local vector control districts, and vector monitoring activities performed by state, federal, tribal, and private entities. 


\section{Protocol and Data Collection}

Sampling will be performed by using a kicknet (or other appropriate sampling device) near vegetation (if present) as part of a multi-habitat assessment for the created impoundments. This method is not comparable to standard dip- cup sampling for larvae and pupae normally performed by mosquitoabatement districts, but potentially is more comprehensive and samples a greater volume. Therefore, this sampling method is expected to provide a conservative estimate of the relative abundance of mosquitoes. Samples will be preserved for later analysis.

Coincident with sample collection, other water-quality and physical habitat attributes will be measured, including water depth, water temperature, dissolved oxygen, and electrical conductivity (salinity). Sample locations will be identified in the field by using GPS.

\section{Data Analysis}

Mosquito larvae and pupae presence or absence and rough density estimates will be determined from the kicknet samples from the created habitats on a monthly basis. This information will be used in conjunction with other information, such as other environmental variables collected during the surveys and by other monitoring teams, to identify important ecological variables that affect abundance of mosquitoes in these habitats. This information will be used to guide management activities for the created impoundments.

\section{Quality Assurance}

As described previously, a detailed monitoring protocol will be developed prior to initiating monitoring activities in the field. This protocol will include a description of the measures that ensure the quality of the data collected and how to implement those measures. The data quality-assurance measures can include, but are not limited to, procedures for calibrating or ensuring the accuracy of any instruments (for example, GPS) employed in the field, procedures for recording and transferring electronic data, methods for ensuring proper operation of field equipment during surveys, and methods for avoiding double counting or insufficient coverage of survey areas.

\section{Data Reporting}

Data will be archived and made accessible from the CDFW's BIOS database when appropriate. Data submitted to the CDFW must be provided in a format suitable for inclusion in the BIOS database (see the "Data Management" section), including appropriate metadata. In addition, an annual progress report will summarize the data collected during that year and update prior reports in a cumulative fashion. At a minimum, the annual report is to include the following:

- GIS maps of the sampling locations.

- Annual and monthly abundance of mosquito larvae and pupae in the created impoundments.

- Environmental attributes collected during the surveys.

- Recommendations for improvement of the survey protocol, if any.

\section{Overview of Similar Current Monitoring Activities}

The Torres Martinez are monitoring mosquitoes in their pilot wetland project. Local vectorcontrol districts also monitor mosquito populations in the Salton Sea area. 


\section{Hydrology and Water-Quality Monitoring}

\section{Overview}

This hydrology and water-quality monitoring plan, which represents one element of the overall Salton Sea monitoring and assessment program, focuses on key activities needed to characterize hydrologic and water-quality conditions at the Salton Sea. Under this monitoring plan, hydrologic conditions include hydrology (surface water and groundwater), hydrodynamics, and meteorological variables; water quality conditions include surface-water, groundwater, and sediment quality. In addition, the hydrology and water-quality monitoring plan includes monitoring of contaminant bioaccumulation that is linked to the concentrations of selenium in the water and sediment.

The water resources of the Salton Sea area are strongly influenced by diversion of water from the lower Colorado River for use in the surrounding area. The source of inflow to the Salton Sea itself is composed almost exclusively of incidental drainage from irrigated areas in the basin, with municipal wastewater flows, runoff and percolation from precipitation in the basin, and groundwater contributing relatively minor amounts. The percentage of surface water inflow to the Salton Sea contributed by Mexico, the Imperial and Coachella Valleys, the local watershed, and precipitation directly on the Salton Sea are shown in table 6.

The Salton Sea is a terminal water body. Evaporation drives water movement out of the lake and is influenced by air temperature, wind speed, relative humidity, salinity, solar radiation, and other factors. Evaporation from the lake represents a substantial water loss, and changing climate conditions could increase evaporation rates over the long term (Roos, 2005).

Water quality in the Salton Sea and its tributaries primarily is influenced by the quality of Colorado River water imported into the watershed and the land-use activities (for example, agriculture) that contribute salts, selenium, nutrients, and other constituents to the Salton Sea inflows (California Resources Agency, 2007). Most of these constituents have become concentrated in the Salton Sea and have contributed to deteriorated habitat conditions over time. Some of these constituents, such as the selenium imported in Colorado River water, could contribute to toxicity risks to the ecosystem and humans through accumulation in the sediment and cycling through the aquatic food webs. Hydrodynamic processes in the Salton Sea also influence water and sediment quality.

\section{Objectives}

The objectives of the hydrology and water-quality monitoring plan fall into two general categories: (1) those related to establishment of existing conditions and documentation of changes over time (trends) and (2) the identification, prioritization, and filling of data gaps that relate to Salton Sea hydrology and water-quality conditions. In general terms, the primary hydrologic inputs and outputs to the Salton Sea and the quality of its water are relatively well known. However, these characteristics are dynamic and changing as a result of local water use (which affects inflows) and the terminal nature of the Salton Sea, which concentrates and stores most of what flows into it. Monitoring hydrology and water quality will reduce uncertainty and improve the ability to predict (through modeling) future conditions related to the size and character of a future Salton Sea. It also could help predict habitat conditions in the Salton Sea and guide decisions related to potential created habitats and air-quality management areas. 


\section{Key Questions and Information Needs}

Key questions to be addressed by the hydrology and water-quality monitoring plan were identified through collaboration with the Hydrology Focused Technical Group, which is a group of experts convened to provide input regarding the monitoring and assessment requirements for hydrologic resources. Key questions were identified in the areas of hydrology, hydrodynamics, water quality, and sediment quality. These key questions were developed in consideration of the constraints and assumptions previously described in the introductory sections of this plan. The full list of key questions identified by the focused technical group is included in appendix 2.

The key questions were evaluated to identify the primary issues and then reviewed to determine whether the question could be answered through monitoring (that is, repeated measurements over time) or through focused investigation (including literature review or assessment of existing information). For those issues that could be answered in whole or in part by monitoring activities, the group identified specific monitoring activities to collect the information needed to answer or help answer the key questions. The specific information associated with answering the key questions are presented in table 7. The specific monitoring activities associated with those key questions are described in greater detail in subsequent sections of this monitoring plan.

\section{Geographic Scope}

The geographic scope for hydrology and water-quality monitoring and assessment is the Salton Sea watershed (fig. 7). The groundwater assessment area includes the groundwater basins with hydrologic connectivity to the Salton Sea. Initially, however, monitoring activities will focus on the Salton Sea itself and at locations within the tributaries that directly contribute flows to the Salton Sea.

\section{Conceptual Model}

The key questions were developed, in part, on the basis of conceptual models relevant to the Salton Sea ecosystem. The conceptual models provide relatively simple depictions of the complex relationships between inputs to the hydrologic system and the processes that drive change. They also serve as starting points for refining the understanding of how the system functions, where data gaps exist, and where additional data collection is needed to reduce uncertainty. The conceptual model developed for hydrology at the Salton Sea is presented in figure 8. Additional conceptual models for selenium, nutrient, and sulfur cycling are presented in appendix 3.

\section{Surface-Water Monitoring Activities}

Hydrologic conditions in the Salton Sea area are expected to continue to change as a result of improvements in irrigation efficiency, transfer of lower Colorado River water outside the Salton Sea watershed, possible increases in evaporation related to climate change, and other factors. Monitoring tributary flows can provide a basis for understanding the relative contribution of different inflows, documenting changes over time, and making predictions (for example, through modeling) of future conditions. This information also is expected to play a fundamental role in future decisions regarding the Salton Sea because of its influence on virtually all of the components (for example, biological resources and air-quality management) of potential restoration. Specifically, accurate hydrologic data allow researchers and decision makers to refine predictions about future changes in hydrology, which could help guide restoration activities and the design or management of created habitats. In addition, these hydrologic data could help assess assumptions in conceptual models and determine whether 
focused investigations are needed to refine those models and to answer key questions regarding hydrologic resources at the lake.

The USGS and the IID currently maintain a flow-monitoring network at the Salton Sea that measures and records flows in the rivers, creeks, and selected direct drains. The inflow monitoring described in this monitoring plan will be integrated with and supplement those ongoing monitoring activities. Additional monitoring sites could be established and operated where needed to meet monitoring objectives. The specific objectives of the surface-water monitoring activities and their intended purposes are presented in table 8 .

\section{Measurement of Tributary Flows (gaging)}

\section{Purpose and Justification}

The fundamental purpose of monitoring the major surface-water inputs to the Salton Sea is to provide a continued record of inflows over time that documents current conditions and provides a basis for projecting future conditions. Inflows to the Salton Sea determine the volume of the lake and its surface elevation, which, in turn, influence water quality, hydrodynamics, habitat quality, and the need for air-quality management. Inflow data, and future predictions of Salton Sea hydrology based on those data, can be useful to answer key questions and clarify what water is available to support potential restoration and management actions at the Salton Sea. In addition, flow-monitoring information can be used to develop a refined water balance, which will be used to predict salinity, nutrient and selenium loading, shoreline elevation, and water depth.

Locations

Primary surface-water inputs include the New, Alamo, and Whitewater Rivers; Salt and San Felipe Creeks; and direct agricultural drains. Flow data collected at the existing USGS gages will be used to monitor flow in the New River (USGS gage \#10255550 near Westmorland) and Alamo River (USGS \#10254730 near Niland). The Whitewater River will be monitored at the current USGS gage (\#10259540) location near Mecca. Similarly, flow information collected at the USGS gage (\#10255550) on Salt Creek will be used; flow-monitoring activities also could include establishment of a flowmonitoring site on San Felipe Creek at or near the location of the discontinued USGS gage (\#10255885). Monitoring also will take advantage of flow information routinely collected by the IID in several direct drains. Additional direct-drain monitoring locations will be identified and established in collaboration with the IID and the Coachella Valley Water District (CVWD).

In addition to these inflow sources, the volume of Salton Sea inflow routed to supply potential created habitats and the discharge from these areas will be measured and accounted for in the overall water budget.

\section{Period and Frequency}

Monitoring of flows in the rivers and creeks initially are proposed to be performed for at least 3 years, after which the need and scale of monitoring can be re-evaluated. The duration of the monitoring, particularly in the direct drains, will depend on the results of initial monitoring and the importance of the continued monitoring to meeting program objectives.

Monitoring of flow in the rivers and creeks will be performed on a continuous (15-minute sampling interval) basis by using data collected at existing USGS gages and from gages installed at new locations as part of the monitoring program. Monitoring of direct-drain flows will be performed at a frequency appropriate for describing the seasonal and annual discharge from those locations. Where 
feasible, monitoring of drain flows will be comparable to monitoring performed in the rivers and creeks. Because of the variability in drain flows, future changes to the monitoring program could provide for less frequent measurement of flow before and after major irrigation activity, with more frequent monitoring performed during field drainage and during peak irrigation season.

\section{Protocol and Data Collection}

It is anticipated that flow data in the rivers and creeks collected at established USGS flow gages (that is, existing USGS automated, continuously recording gages) following USGS protocols (Rantz and others, 1982) will be the primary source of flow data. Any additional gages on rivers or creeks (for example, San Felipe Creek) will be installed and monitored following the standard USGS methodology. The existing USGS gages are permanently installed, and they transmit continuously recorded data by using telemetry. With the exception of the USGS gage at Mecca, real-time, provisional data are available for download from the USGS National Water Information System (http://waterdata.usgs.gov/ca/nwis/nwis). USGS validates the data annually, with adjustments to the stage-discharge rating curves made to account for "shift" that could result from changes in streambed cross sections (Wahl and others, 1995).

Flow measurements in the direct drains can be collected by various means, ranging from manual measurement of depth and velocity at selected cross sections to permanently installed recorders similar to the USGS gages. The techniques and protocols for collection of flow data in the direct drains will be determined in collaboration with the USGS, IID, and CVWD. Flows in direct drains that are pumped to the Salton Sea will be estimated by using a pumping rate based on the rated capacity of the pump (through consultation with the pump operator) and recorded pumping times. Inflow and discharge measurements at created habitats using drain water (rivers or agricultural drains) will be collected following similar protocols.

\section{Data Analysis}

Flow data will be used to describe inflows on a seasonal and annual basis. In conjunction with information on evaporation (see the "Surface Meteorological Monitoring" section) and other losses from the lake, inflow data will be used to calculate the water balance in the Salton Sea and to refine existing hydrologic models. The information gained from this monitoring activity and associated predictions about future conditions is expected to help guide management at the Salton Sea, including the possible construction and management of alternative habitats, such as created saline habitats.

\section{Quality Assurance}

Flow data obtained from USGS gages are assumed to have met USGS quality-control standards once it has been approved for publication. "Real-time" data are provisional until published. Flowmeasurement activities performed by others participating in the monitoring program will be performed in accordance with a quality assurance project plan (QAPP), developed before beginning any monitoring activities. The QAPP will specify staffing, measurement sites, measurement protocols, dataacceptability criteria, quality-assurance/quality-control procedures, data format and databases, and reporting frequencies. The QAPP template (appendix 4) specifies required information for project management, data acquisition, assessment and oversight, and data validation and usability.

\section{Data Reporting}

Data (other than those collected by the USGS) will be archived and made accessible from the CDFW's BIOS database when appropriate. Data submitted to the CDFW must be provided in a format 
suitable for inclusion in the BIOS database (see the "Data Management" section), including appropriate metadata. In addition, an annual progress report will summarize the data collected during that year and update prior reports in a cumulative fashion. At a minimum, the annual report will include the following elements:

- Average daily flow (discharge) for stations with continuous recorders.

- Average monthly flow (discharge) for stations with continuous recorders and field measurements.

- Peak instantaneous flows for stations with continuous recorders.

- Average annual discharge.

- Recommendations for improvement of the monitoring protocol, if any.

Overview of Similar Current Monitoring Activities

The USGS currently owns and operates several gages in the Salton Sea watershed (table 9). The IID also collects drain-flow measurements at a subset of its direct drains.

Measurement of Salton Sea Water-Surface Elevation

Purpose and Justification

Measurement of the water-surface elevation of the Salton Sea is necessary to characterize current conditions and trends and to help answer key questions. In conjunction with bathymetric information, water-surface elevation can be used to quantify the volume and area of the Salton Sea, extent of exposed playa, length of shoreline, depth, and other factors relevant to characterizing existing and future conditions at the Salton Sea.

Locations

Elevation (stage) will be measured at the existing USGS gage (\#10254005) located in the Salton Sea near Westmorland. Depending on the extent of decline in water-surface elevation, an additional gage location could be required during the monitoring period.

\section{Period and Frequency}

Water-surface elevation monitoring at the Salton Sea will be performed over the duration of the monitoring program. Measurement of surface elevation will be performed on a continuous basis at the existing USGS gage.

Protocol and Data Collection

Continuous water-surface elevation measurements are recorded in accordance with USGS protocols.

Data Analysis

Surface-elevation data will be used to describe water-surface elevations at the Salton Sea on a seasonal and annual basis. The information gained from this monitoring activity, in combination with 
data collected through other monitoring activities, is anticipated to guide management at the Salton Sea, including the development of focused investigations and possibly the construction and management of created habitats.

\section{Quality Assurance}

Elevation data obtained from the USGS gage are assumed to have met USGS quality control standards. If the installation of a new elevation gage operated by an entity other than USGS is required because of receding water levels in the Salton Sea in the future, the new gage will be installed in accordance with a QAPP, which will be developed before beginning monitoring activities.

\section{Data Reporting}

Elevation data will be reported by the USGS in its standard format.

\section{Overview of Similar Current Monitoring Activities}

The elevation of the Salton Sea is monitored by both the USGS and IID. The USGS operates one elevation gage, as described previously. Currently, it is a real-time stage data recorder that records elevation measurements on the basis of the National Geodetic Vertical Datum (NGVD) of 1929. Also, the IID currently operates one elevation gage, which is located at Fig Tree John. Readings are recorded at 15-minute intervals, and an automated quality control is performed nightly. Manual quality control is also performed as necessary.

There are differences in the elevation datum and resolution between these two gages. As stated previously, the datum for the USGS gage is based on NGVD29, and all Salton Sea elevations are recorded in feet below mean sea level (msl). The IID elevation gage records to the nearest onethousandth foot (0.001), whereas the USGS data are reported to the nearest one-tenth of a foot $(0.1)$. Based on a comparison of USGS and IID Salton Sea elevation data, there is a difference of approximately 0.9 feet between the two gages (that is, the IID Fig Tree John elevation equals the NGVD29 + 0.911 feet). The USGS gage will be the basis of elevation measurements collected under this monitoring plan. In the event that the USGS gage becomes non-operational, the IID gage at Fig Tree John, a manually read sight gage, or both, will be used as an alternate measure until a new gaging location can be established.

\section{Groundwater Monitoring Activities}

The Salton Sea is a topographically closed depression that is the regional and local sink for surface water and groundwater within the watershed. The lake is surrounded on all sides by waterbearing sediments capable of transmitting groundwater to the lake. Groundwater is also found in sediments underlying the Salton Sea. Upwelling of groundwater from these deeper sediments, as well as groundwater in water-bearing geologic units surrounding the lake, can discharge and mix with waters and sediment within the lake.

Groundwater discharge to the lake is estimated to be about 15,000 acre-feet per year (California Resources Agency, 2006). This represents only 0.01 percent of water discharging to the lake from all sources and a small portion of the overall water budget. However, localized groundwater conditions could influence the formation or stability of salt crusts on the exposed playa. In addition, localized conditions could affect the constructability of restoration facilities (for example, berms and canals), and the location and management of water bodies constructed to support fish and wildlife. 
Groundwater discharge to the Salton Sea generally originates from distinct groundwater basins that are characterized on the basis of the source of watershed recharge to each flow zone, topography in the vicinity of the zone, geology (for example, lithology, faults, and structure), and hydrogeology (water-bearing characteristics of formations and existing groundwater geochemistry). Seven groundwater basins are located around the Salton Sea (fig. 9). Groundwater discharge to the lake and existing groundwater quality in each of these zones are expected to have unique characteristics (California Department of Water Resources, 2003; Tompson and others, 2008). The seven basins follow:

- Coachella Valley Basin.

- West Salton Sea Basin.

- Ocotillo-Clark Valley Basin.

- Imperial Valley Basin.

- Chocolate Valley Basin.

- East Salton Sea Basin.

- Orocopia Valley Basin.

A deep groundwater basin (not shown on figure 9) underlies the identified basins at depths of about 2,000 feet to 20,000 feet below msl (Tompson and others, 2008). This deep groundwater basin is characterized by having no significant groundwater recharge to, or discharge from, surface-water sources or other groundwater basins. As a consequence, the deep groundwater is very old and isolated. This system is also thought to contain the active geothermal resources.

Because groundwater composes a relatively small percentage of the total Salton Sea water balance, detailed characterization of groundwater exchange between the basins and the Salton Sea is a low priority. However, this monitoring plan includes the limited installation of small-diameter wells to monitor shallow-groundwater depth and quality because of the influence shallow groundwater can have on the stability of exposed playa and the role shallow groundwater could have in affecting constructability and water quality of shallow saline habitats.

The specific objectives of the shallow groundwater monitoring activities and their intended purposes are presented in table 10 .

\section{Purpose and Justification}

The purpose of the shallow-groundwater monitoring is to characterize the level and quality of shallow groundwater in the area, particularly in areas where groundwater could interact with exposed playa as the lake recedes. Shallow groundwater could influence the emissivity of particles from sediments on exposed playa, and it could affect the constructability of shallow water habitats and water quality in those habitats. Shallow groundwater also could serve as a water source and salt-management tool for air-quality management and created shallow saline habitats. Understanding the depth and quality of shallow groundwater and how it changes over time will help inform potential habitat creation and air-quality management at the Salton Sea.

The initial shallow-groundwater monitoring will be performed at a scale that will provide preliminary information on shallow groundwater elevation and quality. However, it is anticipated that 
the scale of this effort could be expanded in coordination with a focused investigation on playa stability designed to evaluate a combination of water-level decline, temperature fluctuation, rainfall, sediment composition, water chemistry, and dust emission potential. These activities will be coordinated early to ensure that the objectives of both activities are met.

\section{Locations}

To monitor water levels, shallow, small-diameter groundwater wells with water- surface level data loggers will be installed parallel to the existing northern and southern shorelines of the Salton Sea. Initially, an appropriate number of wells (based on a field evaluation) will be drilled along the -228 -foot contour parallel to the existing shoreline. Additional wells could be drilled farther upslope of -228 feet msl, if it is determined to be necessary. Additional wells could be installed on the exposed playa as the lake recedes in coordination with air-quality studies.

\section{Period and Frequency}

Shallow-groundwater monitoring is to take place over the duration of the monitoring program. The long-term duration of the monitoring will depend on the results of initial monitoring, the rate at which the lake recedes, and the importance of continued monitoring to meeting program objectives.

Shallow-groundwater levels will be measured manually, in addition to those collected by data loggers, on at least a quarterly basis. Groundwater quality samples will be collected quarterly and submitted for laboratory analyses of the constituents identified later.

Alternatively, for elevation data, electronic data loggers could be installed that continuously record groundwater levels. If data loggers are used, data will be retrieved from the loggers monthly, and data quality control will be implemented. Depending on the selected logger, the data could be downloaded manually or remotely, by using cellular phone or satellite.

\section{Protocol and Data Collection}

The small-diameter (1 inch to 4 inch) wells and loggers will be installed according to manufacturer instructions. The depth of the wells will be determined in the field on the basis of the height of observed groundwater. Groundwater depth is anticipated to range from a minimum of 3 feet to a maximum of 15 feet below the playa surface. Well logs will be recorded during the installation of the wells. The elevation of the wells will be surveyed at the time of installation and based on NGVD29. Use of this datum will provide consistency with the USGS Salton Sea elevation- gage data. The elevation benchmark will be clearly marked on the well to ensure that all manual readings measure groundwater elevation from the known datum. If continuous data loggers are used, care will be taken to properly calibrate data loggers to ensure that they record data to the NGVD29 datum.

The small-diameter wells will be of sufficient size to allow for groundwater-quality sampling. Samples will be collected and submitted for laboratory analysis to measure the following constituents:

- Major cations, including calcium, magnesium, sodium, and potassium.

- Major anions, including chloride, sulfate, carbonate and bicarbonate.

- Strontium (cation).

- $\quad$ Borate and fluoride (anions). 
- Sulfide.

- Selenium.

- Nutrients.

These constituents were selected because they are all present in the lake. Because sulfide rapidly converts to sulfate in the presence of oxygen, sulfide only will be measured if the groundwater is anoxic. Therefore, initial samples will include a measurement of dissolved oxygen in addition to the tests for these constituents.

\section{Data Analysis}

Groundwater-elevation data are expected to be tabulated and used to describe shallowgroundwater elevations around the Salton Sea on a seasonal and annual basis. This information will provide a preliminary indication of the potential for shallow groundwater to influence construction activities on exposed playa and to interact with surface sediments. Data collected on the quality of the groundwater, combined with elevation data, can be used to assess the potential for groundwater to interact with surface water in created habitats and to evaluate the potential effects. The water-quality data also could be used to assess the suitability of shallow groundwater as a water source for habitat creation and air-quality management. The shallow-groundwater monitoring activities are expected to be modified in the future on the basis of results of the initial monitoring.

\section{Quality Assurance}

The specific sample-collection and analysis protocols for each parameter of interest in shallow groundwater will be described in an approved QAPP prior to beginning monitoring activities. The protocols will specify bottle type, handling procedures, holding times, preservation, and any special requirements for sample collection. Protocols also will specify the analytical method and acceptance criteria. It is anticipated that the QAPP will be compatible with the SWRCB Surface Water Ambient Water Program (SWAMP; http://www.waterboards.ca.gov/water_issues/programs/swamp/). The QAPP template (appendix 4) specifies required information for project management, data processing and acquisition, assessment and oversight, and data validation and usability.

\section{Data Reporting}

Data will be archived and made accessible from the CDFW's BIOS database when appropriate. Data submitted to the CDFW must be provided in a format suitable for inclusion in the BIOS database (see the "Data Management" section), including appropriate metadata. In addition, an annual progress report will be prepared that summarizes the data collected during that year and updates prior reports in a cumulative fashion. At a minimum, the annual report is to include the following:

- Monthly groundwater elevations for the station(s) with continuous recorders and field measurements.

- Results of laboratory analysis for groundwater constituent concentrations.

- Recommendations for improvement of the monitoring protocol, if any. 


\section{Overview of Similar Current Monitoring Activities}

There are no monitoring programs currently collecting groundwater data. Several public and private agencies or entities have collected groundwater data in the vicinity of the Salton Sea. Some of these data are "point" data that represent a single time or event. Other data are collected as part of monitoring programs. Many of these data are not available in the public domain, but could be available by request. Tompson and others (2008) has synthesized some of these data into a comprehensive dataset.

Several studies have been performed that describe geologic and hydrogeologic conditions in the vicinity of the Salton Sea. Recent studies of interest include the following:

- Groundwater Availability within the Salton Sea Basin (Tompson and others, 2008).

- Coachella Valley Final Water Management Plan (Coachella Valley Water District, 2002a).

- Imperial Irrigation District Water Conservation and Transfer Project, Draft Habitat Conservation Plan, Draft Environmental Impact Report/Environmental Impact Statement (Imperial Irrigation District and U.S. Bureau of Reclamation, 2002).

- Final Program Environmental Impact Report for Coachella Valley Water Management Plan and State Water Project Entitlement Transfer (Coachella Valley Water District, 2002b).

- Salton Sea Ecosystem Restoration Program, Draft Programmatic Environmental Impact Report (California Resources Agency, 2006).

- Geohydrologic Reconnaissance of the Imperial Valley, California (Loeltzand others, 1975).

- Hydrologic Regimen of the Salton Sea, California (Hely and others, 1966).

- Imperial County Groundwater Study Final Report (Montgomery Watson, 1996).

In addition to these recent studies, a variety of older studies or data compilations were developed by the USGS and others.

\section{Hydrodynamics Monitoring Activities}

Hydrodynamic processes at the Salton Sea driven by solar energy, wind, and water inputs appear to be the dominant forces affecting circulation, stratification, and vertical mixing. The geometry of the Salton Basin also affects the hydrodynamics and water quality of the Salton Sea. The Salton Sea contains two subbasins separated by a shallow saddle in the middle. Bathymetry data show that there are relatively flat areas along the southern, southwestern, southeastern, and northern shorelines, and the steepest slopes are along the northeastern shoreline near Salton Sea State Recreation Area. The bottom elevations of the two deepest points in the north and south subbasins are about -278 feet msl.

Local meteorology strongly affects the hydrodynamics of the lake. Meteorological data for California are available from DWR's Office of Water-Use Efficiency, California Irrigation Management Information System (CIMIS). There are five CIMIS stations near the Salton Sea, four of which are active: Salton Sea East (station 127), Salton Sea West (station 128), Westmorland North (station 181), and Calipatria/Mulberry (station 41). The stations measure numerous meteorological values, including 
solar radiation, air temperature, soil temperature, relative humidity, wind speed, wind direction, and precipitation. Additional parameters are calculated on the basis of measured values and include net radiation, reference evapotranspiration, wind roses and wind cubed (an indicator of wind power), vapor pressure, and dew-point temperature. Although the wind fields are complex at the Salton Sea, the strongest winds are generally from the northwest in the north subbasin and from the west in the south subbasin. The maximum wind speeds are greater in the south subbasin.

Water-circulation patterns in the Salton Sea affect the transport and distribution of sediments and nutrients, dissolved oxygen concentrations, mixing of water layers and inflows, temperature gradients, and other water-quality parameters. Energy regimes generated by circulation also have an effect on shoreline erosion and sediment-deposition patterns. Modeling work by the Water Resources and Environmental Modeling Group of the Department of Civil Engineering at the University of California, Davis (Cook and others, 1998) found that wind velocity is the dominant factor affecting water currents in the Salton Sea. The wind pattern results in two large gyres rotating in opposite directions; in the northern basin, currents rotate clockwise, and in the southern basin, the currents rotate counterclockwise (U.S. Bureau of Reclamation, 2000).

The hydrodynamics of the Salton Sea strongly influence the ecosystem and the conditions that lead to events such as fish kills. The relationship among these variables will change as the lake recedes and becomes smaller. Monitoring these conditions in the Salton Sea will help clarify expectations (improved modeling) about the dynamics of a smaller water body, including deep water bodies potentially created as part of restoration. Initially, monitoring of Salton Sea hydrodynamics will focus on activities related to stratification (water-temperature profiles) and currents (water-velocity profiles).

The specific objectives of the hydrodynamics monitoring activities and their intended purposes are presented in table 11 .

\section{Purpose and Justification}

Despite extensive study of Salton Sea water quality, key processes affecting water-quality conditions remain poorly understood. Significant data gaps exist related to meteorological influences on the circulation, mixing, and thermal structure of the Salton Sea. The hydrodynamics are important because they play a role in either exacerbating or improving water-quality conditions in shallow, eutrophic water bodies such as the Salton Sea.

Many of the data necessary to address key questions related to hydrodynamics can be collected as part of related monitoring activities (for example, surface-water hydrology and water and sediment quality). However, data unique to key hydrodynamic questions also are required, specifically, information on thermal stratification and water currents. This type of information is essential to development of hydrodynamic models, which are used to understand stratification, mixing, and turnover.

\section{Locations}

Thermistors and current/velocity sensors will be deployed at a number of sites to measure conditions in the north, south, middle, southwest, and southeast portions of the Salton Sea. For consistency with prior studies, the monitoring sites will roughly correspond to those used by Cook and others (1998), as shown in figure 10. 


\section{Period and Frequency}

Thermistors and current/velocity sensors are to be operated for a minimum of 3 years to provide a continuous dataset for analysis. While deployed, the thermistors and velocity sensors will record data on at least a 15-minute interval. Data collection and equipment maintenance will take place as needed to ensure that the loggers and sensors remain operational.

It is anticipated that data collected through this effort will be analyzed in conjunction with data collected under other monitoring activities to model the stratification, mixing, and circulation dynamics of the Salton Sea. These activities will be coordinated with the ongoing water and sediment-quality monitoring activities to ensure that data are collected in a way that accommodates the objectives of both activities.

\section{Protocol and Data Collection}

Deployment of thermistor chains and co-located current/velocity measuring devices will be undertaken in accordance with an approved QAPP at locations identified spatially by using GPS. Thermistors will be calibrated on deployment and redeployment, and the results of calibration will be recorded. The thermistors will be arrayed at appropriate intervals from the surface to near the bottom of the lake. Current (water velocity) measurements at each location will be made by using equipment or techniques (for example, acoustic Doppler current profilers used by Cook and others, 2002) that provide vertical water-velocity profile information at a minimum resolution similar to the temperature profile provided by the thermistor chain.

\section{Data Analysis}

Temperature profiles and current-velocity data will be used to document thermal and hydrodynamic conditions in the Salton Sea on a seasonal and annual basis. This information, combined with flow, elevation, and climate data, is expected to provide a basis for improved modeling projections of conditions in a smaller Salton Sea or water bodies created as part of potential restoration activities. The information gained from this monitoring activity can help guide management at the Salton Sea in the future.

\section{Quality Assurance}

A QAPP will be developed for the temperature and water velocity monitoring effort. The QAPP template (appendix 4) specifies required information for project management, data processing and acquisition, assessment and oversight, and data validation and usability.

\section{Data Reporting}

Data will be archived and made accessible from the CDFW's BIOS database when appropriate. Data submitted to the CDFW must be provided in a format suitable for inclusion in the BIOS database (see the "Data Management" section), including appropriate metadata. In addition, an annual progress report will be prepared that summarizes the data collected during that year and updates prior reports in a cumulative fashion. At a minimum, the annual report is to include the following:

- A summary of recorded data into daily, monthly, and annual averages.

- Recommendations for improvement of the monitoring protocol, if any. 


\section{Overview of Similar Current Monitoring Activities}

There are no monitoring programs currently collecting continuous water temperatures or watervelocity data. Key existing datasets include temperature and current data collected by Cook and others (1998); temperature profiles measured by Reclamation at 1-meter depth intervals (Holdren and Montano, 2002; Holdren, written commun., 2009); and profiles from thermistors deployed by Cook (2000) at depths of 2.7, 4.1, 7.7, and 14 meters from the surface.

The following studies have evaluated hydrodynamics of the Salton Sea:

- Simulation of wind-driven circulation in the Salton Sea: implications for indigenous ecosystems (Cook and others, 2002).

- SI3D Model application to Salton Sea (California Resources Agency, 2006).

- A linked hydrodynamic and water-quality model for the Salton Sea (Chung and others, 2008).

\section{Water and Sediment Quality Monitoring Activities}

Water and sediment are key drivers of ecosystem function and condition at the Salton Sea. Nutrients (nitrogen and phosphorus), sulfate, and selenium (Se) are among the constituents that, in addition to salinity, threaten the health of the Salton Sea ecosystem (Schroeder and Orem, 2000). These same factors could influence the success of potential created habitats to be constructed at the Salton Sea and the ability of the area to support fish and wildlife over the long term.

The Salton Sea is subject to several dynamic and complex environmental processes and conditions that affect the condition and quality of the water in relation to fish and wildlife habitat at the Salton Sea, including the following:

- Thermal stratification, circulation, and vertical mixing.

- Eutrophication (nutrients and dissolved oxygen).

- Organic production and decomposition.

- Selenium cycling (including bioaccumulation).

- Sulfur cycling.

The measurement of water and sediment quality through this part of the monitoring program can help refine understanding of these processes; however, additional analysis incorporating other data collected through monitoring, and possibly focused investigation, could be required to fully answer key questions about the influence of these processes. Based on the questions and information needs identified in table 7, the following measurement of water and sediment quality and biota were identified for inclusion in the initial monitoring program. Documenting trends in these factors is important for characterizing existing conditions and making decisions regarding potential restoration. The specific objectives of the water and sediment-quality monitoring activities and their intended purposes are presented in table 12. The following sections provide a brief overview of these factors and why monitoring is necessary. 


\section{- Water Temperature}

Water temperature influences the biological and chemical character of the Salton Sea and its tributaries. Temperature measurements taken in the Salton Sea and tributary inflows will be used to develop relationships among climate data (air temperature), warming, and possible thermal stratification to further understand and characterize biological and chemical processes. Water temperature directly influences the biota through physiological stress and indirectly through its effect on chemical processes that influence the biota, such as the solubility of oxygen, the un-ionized fraction of ammonia in the water column, and biological oxygen demand.

\section{- Dissolved Oxygen}

Dissolved oxygen (DO) plays an important role in the quality of aquatic habitat. Eutrophic lakes, including the Salton Sea, regularly exhibit prolonged thermal stratification, which contributes to oxygen depletion, typically during the summer months. Oxygen depletion results when oxygen consumption exceeds oxygen production. Increases in oxygen consumption can be caused by an overabundance of aquatic plants or algae in the ecosystem, wind-driven "turnover" of the water body, high organic loads entering the water, and decay of organic matter (that is, plant or algae die-offs). Because of the high nutrient load and algal density anticipated in the potential shallow water bodies to be created to support fish and wildlife, oxygen depletion could affect the biota. Monitoring dissolved oxygen at the Salton Sea could reduce uncertainty and provide additional information valuable for planning, designing, and managing habitat in the future.

\section{- Secchi Depth}

Secchi depth provides an easy measurement of water clarity, which is a measure of the light attenuation in water attributed to particulates in the water column and, as such, serves as a surrogate measure for total suspended solids and phytoplankton biomass. Both are important characteristics of the overall productivity and water quality.

Secchi depth monitoring would provide a quick, real-time check on the particulate fraction of the water that can be compared to laboratory measures (for example, chlorophyll, total suspended solids) to help identify the causes of waterborne cloudiness. In addition, Secchi depth can provide a quick, costeffective alternative to laboratory samples.

\section{- Electrical Conductivity}

Electrical conductivity is a surrogate measure for salinity and is usually represented as "specific conductivity," which is conductivity normalized to $25^{\circ}$ Celsius (C). Monitoring electrical conductivity provides information on salt content, which can be correlated to laboratory-derived measurements of total dissolved solids. Measurement of electrical conductivity can provide a cost-effective means of increasing the frequency of salinity measurements.

\section{- Total Suspended Solids}

Total suspended solids (TSS) is a gravimetric measure of the weight of dried particles from a known volume of water captured on a pre-dried and weighed filter of a specified pore size that differs from suspended sediment concentration (SSC). TSS is expressed in units calculated from the volume of water filtered, typically as milligrams per liter $(\mathrm{mg} / \mathrm{L})$. Inflow measurements of TSS are an indication of the load of suspended sediment being discharged to the Salton Sea; however, the estimate does not include bed load. Among other uses, monitoring TSS can provide information valuable to determining the need for sedimentation basins and their size. 


\section{- Nutrients}

Phosphorus (total-P), ortho-phosphate $\left(\mathrm{o}-\mathrm{PO}_{4}\right)$, ammonia $\left(\mathrm{NH}_{3}-\mathrm{N}\right)$, nitrate and nitrite $\left(\mathrm{NO}_{3} / \mathrm{NO}_{2} \mathrm{~N}\right)$, and organic nitrogen compose the suite of analyses necessary to characterize loading of the most important nutrients to the Salton Sea. Total phosphorus is a measure of the entire phosphorus concentration in a sample, whereas ortho-phosphate provides an indication of the portion of phosphorus that is biologically available. Total nitrogen is typically measured through the analysis of Total Kjeldahl Nitrogen (TKN), nitrate, and nitrite in a single sample. Together, these three analytes provide the total nitrogen concentration (assuming the TKNs are performed on unfiltered water). Unfiltered water TKN provides results for dissolved and particulate organic nitrogen (plus ammonia). Total nitrogen also can be measured directly with the persulfate oxidation method. Monitoring nutrients in the Salton Sea and its inflows is useful for determining nutrient loads, making predictions about the productivity of created habitats, and determining whether limited treatment of water used for habitat creation could enhance habitat value.

\section{- Sulfate}

High sulfur concentrations in the Salton Sea contribute to the availability of toxic hydrogen sulfide and the formation of turbidity from gypsum flocculation. Monitoring the trends and variability in the sulfate content of the Salton Sea and surface-water inflow can provide important insights into sulfur cycling in the Salton Sea and how created habitats could be affected.

\section{- Total Organic Carbon}

The organic carbon content of aquatic ecosystems is directly related to system productivity and trapping of organic-rich sediments in areas of low transport energy. A buildup of organic compounds in the water column or sediment relates to oxygen-depletion potential and is often strongly associated with contaminants (including selenium and most pesticides). Results for total organic carbon and dissolved organic carbon in water and sediments would be used to track the organic enrichment of the existing environment and any new project features.

\section{- Arsenic}

Arsenic and other trace elements will be standard analytes in water, sediment, and biota samples. Arsenic is known to have toxic effects on biota and has been found to accumulate in Salton Sea fish. Information gained from monitoring arsenic levels in inflow water could be useful for assessing risks and identifying appropriate water sources for created habitats.

\section{- Boron}

Boron will be a standard analyte in water, sediment, and biota samples. Boron is of particular concern for toxicity to plants and possible bioaccumulation. Information gained from monitoring boron levels in inflow water could be useful for assessing risks and identifying appropriate water sources for created habitats.

\section{- Selenium}

Because of its importance, selenium is discussed in detail in a later section of this plan.

\section{- Legacy Pesticides}

Legacy pesticides are those pesticides that were historically used in the watershed, but that have been deregistered for legal use in the United States. These pesticides, which include DDT and its breakdown products, chlordane, and other organochlorine pesticides, have long half-lives in the 
environment, and adsorb strongly to the clay sediments prevalent in the Imperial Valley. These products are rarely found at detectable levels in the water column, but have been observed in sediment and fish tissue. Monitoring for legacy pesticides is useful for determining risk to fish and wildlife and whether sedimentation basins could be used to reduce water-column concentrations of these compounds as they enter the Salton Sea or created habitats.

\section{- Current-Use Pesticides}

Current-use pesticides are those pesticides that are registered for legal use in the State of California and are regularly used. Most current-use pesticides can be classified as organophosphate, pyrethroid, or carbamate pesticides. Organophosphates are typically short-lived in the environment, although they can be toxic to aquatic organisms at very low levels. Carbamate pesticides are more persistent in the environment, and have been shown to sorb to sediments and contribute to sediment toxicity. As described previously, monitoring could be useful for determining risk to fish and wildlife and determining whether sedimentation basins could be used to reduce concentrations of these compounds as they enter the Salton Sea or created habitats.

Legacy and current-use pesticides detected recently in the Salton Sea are summarized in appendix 5. Legacy and current-use pesticides detected recently in tributaries to the Salton Sea are summarized in appendix 6.

\section{Inflow Water and Sediment-Quality Sampling}

Purpose and Justification

Water and sediment-quality monitoring will provide an improved basis for characterizing parameters of concern and the processes that influence the health of the ecosystem. Initially, the water and sediment-quality monitoring program will address a broad range of parameters (described previously) as a means of screening. Once the trends of these parameters are more broadly understood, it is anticipated that the monitoring program will be refined to focus on parameters determined to be most relevant to meeting the monitoring objectives.

\section{Locations}

Water and sediment quality will be sampled at several locations in the Salton Sea watershed. These locations are as follows:

- New River at international boundary.

- New River at outlet.

- Alamo River at international boundary.

- Alamo River at outlet.

- Whitewater River at outlet.

- Selected direct drains.

- Salt Creek. 
- San Felipe Creek.

- All American Canal.

To the extent possible, the water and sediment-quality samples will be collected near the existing flow-measurement gage locations.

Period and Frequency

Monitoring is to be performed for select constituents at specified locations on a continuous, monthly, quarterly, or annual basis. The location, sampling frequency, and constituents that will be collected from water, sediment, and tissue are presented in table 13. Monitoring will be performed for the duration of the monitoring program or until it is determined that the information is no longer needed to meet objectives.

More frequent water-quality monitoring of the rivers and drains could be needed during the peak irrigation season because it is possible that the rapid changes associated with these events are not adequately characterized by monthly monitoring. The frequency of sample collection will be adjusted if initial monitoring indicates that more frequent measurements are necessary.

\section{Protocol and Data Collection}

Table 13 provides a summary of the water quality, sediment quality, and biota monitoring activities to be performed under the hydrologic monitoring program. In general, water-quality samples are a combination of field measurements and grab samples collected at specified intervals and sent to a laboratory for analysis.

Measurements taken in the field, including temperature, dissolved oxygen, turbidity, $\mathrm{pH}$, and electrical conductivity, will be performed by using probes and meters calibrated in accordance with manufacturer instructions and QAPP procedures. It is anticipated that these measurements will be taken throughout the water column (for example, 1-meter intervals). Water samples collected for laboratory analysis will be obtained by using sampling devices and procedures suitable for the specific location and conditions. Sediment samples will be collected by using sampling devices and procedures suitable for the specific location and conditions.

\section{Data Analysis}

Water-quality data collected from inflows would be used to describe and assess trends in waterquality over time. These data, in conjunction with flow-monitoring information, will be used to estimate loading of key constituents, such as nutrients and selenium, and to assess the potential effects of future management actions. This information also is expected to be useful for evaluating the potential for created habitats to support the desired biological function and to help guide the design, construction, and management of shallow and deep-water habitats. Information on the character (for example, grain size) and quality of sediments also can be useful for understanding sediment transport and contaminant dynamics as well as evaluating air-quality effects associated with exposed playa and planning airquality management actions.

\section{Quality Assurance}

The specific sample collection and analysis protocols for each parameter of interest will be described in an approved QAPP prior to commencement of monitoring activities. The protocols will specify bottle type, holding procedures, holding times, preservation, and any special requirements for 
sample collection. Protocols also will specify the analytical method, detection limits, and acceptance criteria. Sediment and biota sampling will be specified in the QAPP. It is anticipated that the QAPP will be compatible with the SWRCB's SWAMP. The QAPP template (appendix 4) specifies required information for project management, data processing and acquisition, assessment and oversight, and data validation and usability.

\section{Data Reporting}

Data will be archived and made accessible from the CDFW's BIOS database when appropriate. Data submitted to the CDFW must be provided in a format suitable for inclusion in the BIOS database (see the "Data Management" section), including appropriate metadata. In addition, an annual progress report will be prepared that summarizes the data collected during that year and updates prior reports in a cumulative fashion. At a minimum, the annual report is to include the following:

- A summary of data collected.

- Monthly, seasonal, and annual trends in constituent concentrations.

- Recommendations for improvement of the monitoring protocol, if any.

Overview of Similar Current Monitoring Activities

Reclamation currently performs quarterly water-quality monitoring at three locations: Alamo River outlet, New River outlet, and Whitewater River outlet. Field measurements are recorded for temperature, DO, turbidity, $\mathrm{pH}$, and electrical conductivity. Grab samples are collected and submitted for laboratory analyses for nutrients (total phosphorus, ortho-phosphate, ammonia, nitrate, nitrite, and TKN); total selenium, selenate, and selenite; and total suspended solids (Holdren and Montano 2002; Holdren, unpublished data).

The IID currently has two major water-quality monitoring programs: the Drain Water Quality Improvement Program and the environmental mitigation program associated with the IID/San Diego County Water Authority water transfer. Under the Drain Water Quality Improvement Program, the IID monitors 7 major drains on a monthly basis and 18 minor drains on a quarterly basis. In addition to field parameters (temperature, DO, turbidity, $\mathrm{pH}$, and electrical conductivity), the IID submits samples for TSS, turbidity, total phosphorus, ortho-phosphate, nitrate, nitrite, ammonia, and TKN.

\section{Salton Sea Water and Sediment-Quality Sampling}

\section{Purpose and Justification}

Water and sediment-quality monitoring in the Salton Sea provides an improved basis for identifying constituents of concern and better defining the processes that influence the health of the ecosystem. Initially, the water and sediment-quality monitoring program will address a broad range of parameters (described previously) as a means of screening. Once the trends of these parameters are broadly understood, it is anticipated that the monitoring program could be refined to focus on parameters determined to be most relevant to meeting the monitoring objectives.

Locations

Locations selected for monitoring in the Salton Sea are listed in table 13. These locations include the monitoring sites currently used by Reclamation in its quarterly monitoring program. 


\section{Period and Frequency}

Monitoring of the parameters listed in table 13 will be performed for the duration of the monitoring program or until it is determined that the information collected is no longer needed to meet the monitoring objectives. Water quality and sediment quality monitoring of the Salton Sea will be performed at the frequency identified for each parameter in table 13.

\section{Protocol and Data Collection}

In general, water-quality samples will include a combination of field measurements and grab samples collected at specified intervals and sent to a laboratory for analysis. Measurements taken in the field, including temperature, $\mathrm{DO}$, turbidity, $\mathrm{pH}$, and $\mathrm{EC}$, will be made by using probes and meters calibrated in accordance with manufacturer instructions and QAPP procedures. It is anticipated that these measurements will be taken throughout the water column (for example, 1-meter intervals). Water samples collected for laboratory analysis will be obtained by using sampling devices and procedures suitable for the specific location and conditions. Sediment samples will be collected by using sampling devices and procedures suitable for the specific location and conditions.

\section{Data Analysis}

Data collected under this element of the monitoring program will document current conditions and long-term trends in inflow water quality. This is expected to contribute to developing models or projections of future conditions that will help estimate future loading and guide management actions. In addition, it is expected that this information will be used in conjunction with hydrodynamic and climate data to model the eutrophic conditions of the lake, and to provide the basis for a better understanding of

processes such as carbon and sulfur cycling in the Salton Sea and how these processes influence habitat conditions.

\section{Quality Assurance}

The specific sample collection and analysis protocols for each parameter of interest will be described in an approved QAPP prior to commencement of monitoring activities. The protocols will specify bottle type, holding requirements, holding times, preservation, and any special requirements for sample collection. Protocols also will specify the analytical method, and acceptance criteria. Sediment and biota sampling specific to location will be specified in the QAPP. It is anticipated that the QAPP would be compatible with the SWRCB's SWAMP. The QAPP template (appendix 4) specifies required information for project management, data processing and acquisition, assessment and oversight, and data validation and usability.

\section{Data Reporting}

Data will be archived and made accessible from the CDFW's BIOS database when appropriate. Data submitted to the CDFW must be provided in a format suitable for inclusion in the BIOS database (see the "Data Management" section), including appropriate metadata. In addition, an annual progress report will be prepared that summarizes the data collected during that year and updates prior reports in a cumulative fashion. At a minimum, the annual report is to include the following:

- A summary recorded data.

- Monthly, seasonal, and annual trends in constituent concentrations. 
- Recommendations for improvement of the monitoring protocol, if any.

\section{Overview of Similar Current Monitoring Activities}

Reclamation staff have been monitoring water quality quarterly since 2004 (Holdren, unpublished data). They collect samples from the outlets of the New, Alamo, and Whitewater Rivers and from three sites in the Salton Sea. Reclamation monitors quarterly at three locations: SS-1 (North Basin), SS-2 (mid-lake), and SS-3 (South Basin). Field measurements are recorded for temperature, DO, turbidity, $\mathrm{pH}$, and electrical conductivity. Grab samples are collected and submitted for laboratory analysis for nutrients (total phosphorus, ortho-phosphate, ammonia, nitrate, nitrite, and TKN), total selenium, selenate, selenite, total suspended solids, Secchi depth, and chlorophyll. Additionally, sediment samples are collected and analyzed for total selenium.

\section{Selenium Sampling (water, sediment, and biota)}

Water diverted from the lower Colorado River to support irrigated agriculture and other uses in the Imperial and Coachella valleys contains selenium derived from sources in the Colorado River watershed (California Resources Agency, 2007). Elevated concentrations of selenium have been measured in the Salton Sea ecosystem. Inorganic forms of selenium (selenate and selenite) usually predominate in water, but inorganic and organic forms of selenium are found in water, sediment, and biological tissues (see appendix 3). In aquatic systems, most selenium is generally associated with sediments (acting as a sink and reservoir) or plants and animals. In water, selenate is reduced to selenite, and both forms are removed from the aqueous phase into sediment (Tokunaga and others (1996). Once in sediment, the selenite is reduced to elemental selenium, which can make up 99 percent of the selenium found in sediments.

Influent riverine total selenium concentrations to the Salton Sea are commonly in the 5 to 10 micrograms per liter $(\mu \mathrm{g} / \mathrm{L})$ concentration range and are almost evenly divided between selenate and selenite (Setmire and Schroeder, 1998). On reaching the Salton Sea, the waterborne selenium is rapidly reduced to less than $2 \mu \mathrm{g} / \mathrm{L}$ and is essentially all found as selenite or organic selenium (Schroeder and others, 2002). Salton Sea waterborne selenium appears to be rapidly assimilated into biota, which becomes part of an organically rich sediment, particularly in the deepest portions of the Salton Sea (Setmire and Schroeder, 1998).

The current, frequently anoxic character of deep sediments in the Salton Sea acts to lock up most of the Salton Sea's selenium, so it is biologically unavailable (Schroeder and others, 2002). This finding has been confirmed by experiments with Salton Sea sediment cores used to measure selenium flux under oxygenated and anoxic conditions (Byron and Ohlendorf, 2007). It is hypothesized that watercolumn selenium concentrations could increase to as high as $400 \mu \mathrm{g} / \mathrm{L}$ (from a current average near 1 $\mu \mathrm{g} / \mathrm{L}$ ) if not retained in the low redox sediments as reduced, insoluble compounds (Schroeder and Orem, 2000). The resuspension of these selenium- and phosphorus-rich bottom sediments appears to be a key factor causing and perpetuating the Salton Sea's highly eutrophic character (Anderson and Amrhein, 2002; Rueda and Schladow, 2003), which, in turn, acts to maintain the anoxia of bottom waters and the conversion of sediment selenium to relatively insoluble reduced species.

Selenium in the Salton Sea and surface inflows has the potential to reach concentrations that produce deleterious effects in fish and wildlife. Humans who consume fish and other animals from the Salton Sea also could be adversely affected. The extent of these effects depends on a variety of factors that include the amount of selenium that enters the Salton Sea and how it cycles through the ecosystem. 


\section{Purpose and Justification}

Monitoring selenium in the Salton Sea, surface inflows, and the biota is needed to evaluate the ecological and human risk associated with selenium in the Salton Sea and created habitats that could be constructed to support fish and wildlife. This information and associated risk analyses will determine the ecological and human health risks related to selenium in the Salton Sea. This information also will help guide the placement, design, and management of created habitats to minimize risks.

\section{Locations}

Selenium sample collection will be co-located with the inflow water and sediment samples at the outlets of the New, Alamo, and Whitewater Rivers. In the Salton Sea, the samples will be co-located with water, sediment, and invertebrate water-column and benthos samples. These will be collected from about 10 deep-water (greater than 3 meters) locations (five in the north basin and five in the south basin) and about 10 shallower water (less than 3 meters) locations (three in the north, three in the south, and two each from the east and west shorelines). These will be the same samples collected for the purpose of monitoring benthic and water-column invertebrates. To the extent possible, these samples will be collected near locations where fish monitoring is performed so that fish tissue samples can be obtained at the same time and selenium concentrations can be interpreted across media.

\section{Period and Frequency}

Monitoring is to continue for the duration of the monitoring program or until it is determined that continued monitoring is not needed to meet program objectives. Samples will be collected through the year at the frequency identified in table 13.

\section{Protocol and Data Collection}

The protocols for selenium sampling will follow those identified for water and sediment sampling; the invertebrate and fish sampling will be performed as part of the biological monitoring. Fish tissue analyses will be performed on small fish (whole body) to evaluate ecological risks and on fillets from larger tilapia for the purpose of obtaining information that could be used to evaluate human-health risk.

\section{Data Analysis}

Data collected under this element of the monitoring program will be used to document selenium levels in the various media and trends in selenium loading. The data also are anticipated to be used, in conjunction with data from other monitoring activities, to predict future loading and evaluate the ecological and human risk associated with selenium in the Salton Sea and created habitats that could be constructed to support fish and wildlife.

\section{Quality Assurance}

The specific sample collection and analysis protocols for selenium in each medium (water, sediment, tissue) and location will be described in an approved QAPP prior to commencement of monitoring activities. The protocols will specify bottle type, holding requirements, holding times, preservation, and any special requirements for sample collection. Protocols also will specify the analytical method, and acceptance criteria. 


\section{Data Reporting}

Data will be archived and made accessible from the CDFW's BIOS database when appropriate. Data submitted to the CDFW must be provided in a format suitable for inclusion in the BIOS database (see the "Data Management" section), including appropriate metadata. In addition, an annual progress report will be prepared that summarizes the data collected during that year and updates prior reports in a cumulative fashion. At a minimum, the annual report is to include the following:

- Monthly, seasonal, and annual trends in selenium concentrations in water, sediment, and tissue.

- Recommendations for improvement of the monitoring protocol, if any.

\section{Overview of Similar Current Monitoring Activities}

As part of its mitigation monitoring requirements associated with the water transfer, the IID is currently monitoring and assessing selenium and its potential effects on desert pupfish occupying agricultural drains. IID is working in collaboration with Reclamation and the USGS. Monitoring funded by the IID and performed by the USGS assessed selenium in the water column of 29 direct drains and sediments (May and others, 2007; Saiki, 2007, 2008, 2010). In seven of the drains, more intensive sampling and analyses was performed during April and October. Water samples were analyzed for selenium (including speciation in the April and October samples from the seven drains) and suspended solids. Sediment, detritus, algae, plankton, midge larvae, and fish (western mosquitofish and sailfin mollies, as surrogates for desert pupfish) samples from the seven drains selected for intensive sampling were analyzed for selenium in April and October. Sampling was initiated in August 2005 and continued through April 2009.

Reclamation staff has been monitoring water-column selenium quarterly since 2004 (Chris Holdren, unpublished data). They collect samples from the outlets of the New, Alamo, and Whitewater Rivers and from three sites in the Salton Sea. Reclamation performs quarterly monitoring at three locations: SS-1 (North Basin), SS-2 (mid-Sea), and SS-3 (South Basin). Sediment samples are also collected and analyzed for total selenium. 


\section{Geography and Geology}

\section{Overview}

Geography and geology monitoring, which represents one element of the overall Salton Sea MAP, focuses on key activities needed to characterize geographic and geologic conditions at the Salton Sea. This section describes monitoring activities related to the regular or periodic collection of geographic and geologic information. Geographic conditions refer to the landscape or surface characteristics of the study area that will be important in assessing changes to the landscape as a result of restoration. These characteristics include bathymetry, land- surface elevation, and land use. Geologic conditions refer to the geological processes in the study area, including landslides and slumps, sedimentation rates, and seismic activity.

\section{Objectives}

The objectives of the geography and geology monitoring follow directly from the overarching goals and objectives identified for the MAP and fall into two general categories: 1) those related to establishment of existing conditions and documentation of changes over time (trends), and 2) the identification, prioritization, and filling of data gaps that relate to geographic and geologic processes. The establishment of existing conditions is intended to help assess effects or changes resulting from potential restoration activities and guide the design and management of these restoration actions.

\section{Key Questions and Information Needs}

Key questions to be addressed by geography and geology monitoring were identified through collaboration with the Geography and Geology Focused Technical Group, which is a group of experts convened for the purpose of providing input to the monitoring and assessment requirements for geographic and geologic resources. These key questions were developed in consideration of the constraints and assumptions described in the introductory sections of this plan.

The key questions were evaluated to identify the primary issues and then reviewed to determine whether the question could be answered through monitoring (that is, repeated measurements over time) or through focused investigation (including literature review or assessment of existing information). For those issues that could be answered in whole or in part by monitoring activities, the group identified specific monitoring activities needed to collect the information to answer or contribute to answering the key questions (table 14).

\section{Geographic Scope}

The assessment area for geography includes boundaries for surface water (watersheds) and groundwater because these components affect inflows into the lake, and, therefore, bathymetric elevations (see figure 7). Most of the geographic monitoring would be focused on the Salton Sea watershed and the lake itself. The assessment area for geology and seismicity is defined as the geographical area within which the large majority of effects are expected. Fault zones within the Salton Sea region are shown in figure 11. 


\title{
Conceptual Model
}

As described for other elements of the MAP, conceptual models and working hypotheses will be developed prior to initiating any monitoring activities related to geography and geology.

\section{Geography Monitoring Activities}

\author{
Bathymetry of the Salton Sea
}

\section{Purpose and Justification}

Bathymetric data provide topographic relief information for submerged lands and are used to define the elevation, area, and volume relations that are necessary to understand the effects of natural or human-induced changes to the lake. The planning, design, and construction of restoration actions that could be implemented within the Salton Sea or on exposed lakebed (for example, created, shallow, saline water bodies) require high resolution bathymetric information. Currently, the quality of available bathymetric information is insufficient to thoroughly assess potential restoration actions, plan and design restoration features and facilities, and monitor changes to the lakebed. For example, detailed bathymetric data could help project the amount of lakebed playa that would be exposed as a result of declines in water-surface elevations. Depending on the method of collection, bathymetric data can be used to generate depth contours or to create a digital terrain model of the lake bottom.

Bathymetric data are necessary for planning purposes related to future construction, environmental management, wildlife monitoring, and lake recreation. These data can also assist in water-budget calculations, help to develop and calibrate salt-balance models, and facilitate business projections for industries that utilize the lake. Bathymetric surveys can provide important information on the physical characteristics of the lake that will be useful for predicting air-quality effects from exposed playas and for informing management in the design of restoration components.

A recent bathymetric survey by Scripps Institution of Oceanography combining the results of multiple techniques was completed in 2011. Reclamation and the USGS have performed bathymetric surveys several times over the past 20 years. However, the bathymetric characteristics can change as a result of water and sediment inflows, and movement of particles on the lake bed due to currents within the Salton Sea and wind. This is especially true in the shallow areas near the southern and northern shorelines.

\section{Locations}

Bathymetric data will be collected for the entire lake bed and any adjacent or related ponds or bodies of water. This information can be used to design hydraulic facilities that rely on gravity to convey water through created shallow ponds and to establish a basis for design layouts for all facilities. Initially, however, bathymetric data will be collected near the current shoreline areas in selected locations where created shallow ponds are proposed.

\section{Period and Frequency}

The bathymetry of the lake will be determined initially to establish existing conditions and as a basis for design of facilities. Thereafter, monitoring of the bathymetry will take place only as needed to determine whether the lake bed bathymetry has changed. The need for future monitoring will be determined by the stability of the lake bed. The frequency of repeated measurements could be higher in shallow areas, where Sea bed topography is most influenced by wave action and sedimentation; deeper 
areas probably do not change as rapidly and could require less frequent monitoring. In addition, the frequency of monitoring will be dependent on whether any changes are known or suspected of taking place beneath the surface because of such things as construction or natural hazards (slumps, landslides, earthquakes, or sedimentation).

Protocol and Data Collection

Bathymetric data are typically collected by using a sonar sensor mounted on a vessel. To monitor bathymetry of the lake, an automated system, composed of a digital and analog (paper chart) single-beam fathometer, coupled to a real-time, differentially-corrected global positioning system (RTDGPS) system with the data logged into a navigational computer, could be used for the basic position and depth data collection in the lake. Data will be collected for the navigable areas of the lake in both digital and analog (paper chart) format at a specified east-west transect length interval and additional quality control north-south "tie" transects. Although many deep-water lakes are surveyed with multi-beam systems, such systems do not adequately correct for changes in sound velocity throughout the water column and are of limited utility in shallow, saline waters. Therefore, depths to the bottom of the lake will be obtained by using equipment that measures the time required for a sonar signal to travel from the transmitter to the bottom of the lake and back to the receiver. This equipment measures sound velocities (by using a direct time-of-flight sound velocity sensor) and calculates corrections to the basic time-of-travel data, which provides a velocity-corrected distance between the transmitter and receiver. Distance from lake-surface to the transceiver face is measured and added to the distance between the transducer and lakebed to calculate bed depth.

Collecting bathymetric data directly along a shoreline is complicated by the difficulty of navigating a boat in shallow water. There are experimental airborne devices that can collect bathymetric data to moderate depths and simultaneous topographic data immediately above the shoreline. One such device is the Experimental Advanced Airborne research Lidar (EAARL) sensor, which can be used to collect bathymetric details in shallow water along with the topography of the adjacent shoreline. Lidar refers to 'light detection and ranging' and uses a laser that measures the distance from the sensor to the ground. The Lidar technology used by EAARL is discussed in the Land Surface Elevation section. A key challenge in the use of Lidar at the Salton Sea is the lack of water clarity caused by primary productivity. Lidar works best in water with good visibility.

\section{Data Analysis}

Bathymetric survey results will be used in conjunction with other information, such as information on other environmental variables collected during the surveys and by other monitoring teams, to assist in planning related to future construction, environmental management, wildlife monitoring, lake recreation, and the development of focused investigations. Bathymetric data can also assist in water-budget calculations, help to develop and calibrate salt-balance models, and facilitate business projections for industries that utilize the lake.

\section{Quality Assurance}

Initial processing of the data will include the manual removal of spurious data points (outliers) such as single-point depths located significantly above or below the general lake bottom trend, zero depths, returns from fish or suspended debris in the water column, or data that show roll or vertical boat movement. The resultant cleaned data files will then be corrected for sound velocity by applying the sound-velocity profile data to the raw depths collected during the survey. Depth from the lake surface to the active face of the transducer is added to the sound-velocity-corrected depths to calculate depth 
below water surface. Daily average lake-altitude data (based on the daily recorded lake altitude) will be used to adjust the processed depth below lake-surface values to altitude. The data points defining the bottom surface of the lake will be compiled into a single data file, combined with available near-shore bathymetric data, and processed into a triangular irregular network (TIN) for contour generation. Compilation of the data into a single data layer would allow for integrative efforts with other (for example, Lidar-based) elevation data.

\section{Data Reporting}

Data will be archived and made accessible from the CDFW's BIOS database when appropriate. Data submitted to the CDFW must be provided in a format suitable for inclusion in the BIOS database (see the "Data Management" section), including appropriate metadata. In addition, an annual progress report will be prepared that summarizes the data collected during that year and updates prior reports in a cumulative fashion. At a minimum, the annual report is to include the following:

- A summary of data collected during the year.

- Maps depicting updated bathymetric contours.

- Recommendations for improvement of the monitoring protocol, if any.

Overview of Similar Current Monitoring Activities

There are no monitoring programs currently collecting bathymetric data in the Salton Sea region.

\section{Land-Surface Elevation and Lidar}

\section{Purpose and Justification}

Elevation data are used in the production of accurate digital imagery. Elevation data are also used in the production of flood hazard maps, watershed delineations, land-cover classifications, and soil surveys. Elevation data are also used for transportation and utility planning, slope determination, structures placement, and visual analysis. Land-surface elevation is strongly correlated to surface water and the distribution of water bodies and streams. Elevation data provide valuable information for ascertaining hazard susceptibility and are useful in construction planning, modeling runoff and water flow, defining and predicting circulation patterns, and in planning related development.

High-resolution elevation data are often provided as a bare earth digital elevation model. The elevation models can be used to determine heights, slopes, aspect, lines of sight, watercourses, basin capacity, cut-and-fill volumes, relief, and other positional components that can be input for monitoring processes. Some uses include calculating surface areas that are likely to be exposed (or hidden) as water level changes, amounts of material needed for fill, and susceptibility of slopes to geologic hazards.

Locations

Elevation data are needed for the Salton Sea shoreline and areas extending inland, where restoration facilities or staging areas could be located. Elevation data will be collected as close to the water's edge as possible to ensure that elevation and bathymetry data can be combined into a single land-surface elevation dataset that extends beyond the current shoreline at the lake. 


\section{Period and Frequency}

Land-surface elevations will be determined initially to establish existing conditions and as a basis for design layouts for all facilities. In general, land-surface elevations change infrequently, so, typically, elevation data are not updated at regular intervals. Land-surface elevations will be updated when elevations are known to have changed because of development or natural processes, such as landslides or earthquakes. It could also be useful to collect new elevation data when more precise methods of data collection are introduced and the extra level of precision is desired.

\section{Protocol and Data Collection}

Elevation data around the lake have been available through 1:24,000-scale US Geological Survey topographic maps. The data are depicted as contour lines and were derived from stereo-aerial photography. The contour-line interval in the Salton Sea area is 10 feet and the data are quite detailed. However, these elevation data are based on topographic input that is frequently over 20 years old. Newer data are needed because of the age of the topographic maps, subsidence around the lake, and ongoing seismic activity. New technology is available that can provide detail beyond the elevation extraction methods employed years ago.

In 2010, USGS released a contract to collect new elevation data for a 5-kilometer radius around the Salton Sea shoreline. A Lidar airborne sensor was used to collect the data. Lidar refers to 'light detection and ranging' and uses a laser that measures the distance from the sensor to the ground. Airborne Lidar sensors are able to send and receive thousands of laser pulses per second over a swath beneath the aircraft. The laser returns show the locations and heights of anything it encounters, including the ground, vegetation, and manmade structures. Data were collected November 9-13 to coincide with the annual minimum lake elevation so that as much shoreline was exposed as possible. The resulting project produced high-resolution data for a 367-square mile region surrounding the lake. Ground-based Lidar could be used in the future, but its application is generally limited to small areas relative to the requirements of this project.

\section{Data Analysis}

To collect the data, a project "Task Order" was developed to provide details on the collection criteria. The relatively flat terrain around the Salton Sea, sparse vegetation, and generally clear flying conditions provide a good opportunity to collect a very detailed elevation dataset. Specifications were based on "U.S. Geological Survey National Geospatial Program Base Lidar Specification, Version 13" (ILMF-- http://lidar.cr.usgs.gov/USGSNGP\%20Lidar\%20Guidelines\%20and\%20Base\%20Specification\%20v13\%28ILMF\%29.pdf). The nominal point density was five points per square meter with a target vertical accuracy of $\pm 9.25 \mathrm{~cm}$. This vertical accuracy is very high and permits the generation of 1-foot contour intervals, if desired. The specifications document provides details on other collection factors, including a requirement for hydro flattening. Hydro flattening assigns a single elevation value to the shorelines of large water bodies to eliminate varying elevations within the water body.

Data were acquired in nine separate flight missions. The target flying height for the project was 800 meters previously mean terrain. Prior to the flights, airborne GPS base stations, control, and check points were established. The Lidar sensor system was calibrated and kinematic GPS and inertial measurement unit data were collected during the flights to calculate the exact location of the aircraft. Once the collection was complete, the data were digitally processed to generate the project deliverables.

Project deliverables included the following: 
- Project metadata with details about the dataset.

- Raw point cloud - all data points collected by the sensor, with X, Y, Z locations and date/time stamp.

- Classified point cloud - a copy of the point-cloud data with each point assigned to a specific class (unclassified, bare-earth, noise, water, ignored ground).

- Bare-earth surface model — a digital elevation model (DEM) showing the ground surface without ground features, based on a 1-meter grid.

\section{Quality Assurance}

Lidar data collection needs to undergo a number of quality-control and quality-assurance practices. The array of data points collected by the airborne Lidar sensor must be tied to known points on the ground, thus well surveyed points and control are needed before and during the flights. Once the data are collected, quality assurance involves ascertaining the accurate horizontal and vertical positioning of the Lidar points. A major element of this evaluation is to compare the Lidar to known reference points or surveyed points. Other steps in quality assurance include ensuring alignment of flight lines and reducing systematic errors such as image striping.

The Salton Sea Lidar data collection included an additional quality concern. There are currently a number of well-established control points across the country, which are maintained by the National Geodetic Survey and other local, state, and federal agencies, as well as private surveying control networks. These points are typically employed to help establish a fixed location reference for new Lidar-derived data points. Ground control points are available around the Salton Sea, but the area is subject to subsidence so the ground control needed to be corrected prior to any Lidar points being tied to the control. This was accomplished by the USGS vendor to ensure the new elevation data would be as accurate as possible.

Once the Lidar data have been collected and products developed, the data are tested to ensure they meet specifications. A major indicator in Lidar quality is the vertical accuracy of the data. The project included a surveying team to independently collect points across the project area. Project specification called for vertical root mean square error (RMSEv) not to exceed 0.0925 meters $(\mathrm{m})$. The RMSEv of the review checkpoints was $0.035 \mathrm{~m}$, indicating that the data collected surpassed the standard. Other QA procedures checked for proper data formats, spatial reference, data density and data voids, and proper feature removal from the bare-earth model.

\section{Data Reporting}

All data developed through the project are available in the public domain. The bare-earth digital elevation model (DEM) was incorporated into the National Elevation Dataset at a reduced resolution of 1/9 arc-second (approximately a 3-meter grid) and is available for download (see:

nationalmap.gov/viewer). The raw point cloud, original 1-meter DEM, and other deliverables are all available from the USGS. Extensive report documentation explaining the data collection, QA processes, and establishment of ground control is also available.

Data will be archived and also made accessible from the CDFW's BIOS database when appropriate. Data submitted to the CDFW must be provided in a format suitable for inclusion in the BIOS database (see the Data Management Section), including appropriate metadata. In addition, an 
annual progress report will be prepared that summarizes the data collected during that year and updates prior reports in a cumulative fashion. At a minimum, the annual report is to include the following:

- A summary of data collected during the year.

- Maps depicting updated land surface elevations.

- Recommendations for improvement of the monitoring protocol, if any.

Overview of Similar Current Monitoring Activities

The new Lidar dataset will serve as a new topographic base for the Salton Sea region. Elevation data usually are not updated frequently because of relatively infrequent change in terrain. The Salton Sea elevations are subject to change as a result of seismic activity, subsidence, and terrain modification due to construction work near the lake. These factors could require Lidar updates in the future to keep the data current.

\section{Land Cover}

\section{Purpose and Justification}

Land-cover data enhance the ability to use and interpret other base-map datasets, such as watersheds, soil surveys, flood hazards, transportation, geothermal resources, and critical infrastructure. Monitoring land use helps document spatially explicit alterations and transformations of the natural resource base. Land-cover data are needed to understand how humans (and climate) are modifying the land resources and to help predict some of the fundamental effects of these changes on the environment and humans.

\section{Locations}

Land-cover data will be collected in areas around the Salton Sea, including areas affecting the Salton Sea (for example, adjacent mountains or agricultural areas) and those affected by it (for example, downwind development).

\section{Period and Frequency}

Land cover around the lake will be determined initially to establish existing conditions and as a basis for design of facilities. Land-cover data are typically collected at regular intervals that depend on the amount of change anticipated. If rapid land-cover changes are expected around the lake from new development or changes in agriculture, then annual or biennial collections could be desired. The latest Nationwide Land Cover Database (NLCD) was derived from 2006 Landsat images. Further updates are expected about every 5 years. If additional land-cover detail or more frequent updates are needed for the Salton Sea, then land-cover data in the Salton Sea region could be collected more frequently at a higher resolution than the NLCD.

\section{Protocol and Data Collection}

Land cover is generally determined at one point in time, usually from an aerial photograph or satellite image. Land-cover data for the Salton Sea region can be downloaded from a regularly updated data source like the NLCD. The California Department of Water Resources (DWR) also collects geographic surveys of agricultural lands on an intermittent basis. New land-cover data can also be 
obtained under the MAP through aerial photography or from satellite images (see section on "Orthoimagery").

Data Analysis

The NLCD provides national coverage that is well positioned to address regional needs. The data are derived from Landsat data, which means the source-data cell size is 30 meters. This suitable for land-cover data creation at a scale of about 1:100,000. The images are classified into a set of 16 distinct landcover categories (such as forest, grassland, urban, agriculture, and so on). Once lands are classified into general categories, the data can be used as input in other Salton Sea management tasks.

Quality Assurance

Quality-assurance methods are well established for land-cover datasets. The Multi-Resolution Land Characteristics Consortium (MRLC) is a multi-agency group that purchases Landsat imagery and coordinates the production of a comprehensive land-cover database (NLCD). NLCD data collections follow established standards; however, these can be modified between successive releases. Standards for the latest NLCD release based on 2001 data can be found at www.mrlc.gov/pdf/July_PERS.pdf.

Quality assurance is performed by comparing the placement of the pixels by automated methods against reference data. The reference data can be collected in the field or be derived from other imagery of the target area at (typically) a higher resolution than was used to generate the original land cover. Errors are presented as errors of commission (extra pixels erroneously identified as a particular class - a false positive) or errors of omission (features that exist in the field but were not mapped by the imagery).

\section{Data Reporting}

Data will be archived and made accessible from the CDFW's BIOS database when appropriate. Data submitted to the CDFW must be provided in a format suitable for inclusion in the BIOS database (see the "Data Management" section), including appropriate metadata. In addition, an annual progress report will be prepared that summarizes the data collected during that year and updates prior reports in a cumulative fashion. At a minimum, the annual report is to include the following:

- A summary of data collected during the year.

- Maps depicting updated land cover information.

- Recommendations for improvement of the monitoring protocol, if any.

Overview of Similar Current Monitoring Activities

Existing NLCD datasets from 1992 and 2001 are available, and a dataset based on 2006 imagery is in progress. The CDFW is working on a statewide vegetation map. DWR completed land-use surveys for Imperial County in 1997 and for Riverside in 2000

(see: www.water.ca.gov/landwateruse/lusrvymain.cfm). 


\section{Orthoimagery}

\section{Purpose and Justification}

The collection of aerial images in the Salton Sea study area is of great importance for monitoring conditions at the lake and the surrounding environment. Depending on the analysis needed, images can be a primary data resource because many other datasets depend on high-quality images. Transportation, land cover, hydrography, structures, and soil surveys all depend on accurate imagery.

Images (photos) obtained from aircraft or satellites are available as "orthoimages." An orthoimage is an image that has been corrected to remove displacements caused by terrain relief and sensor tilt and is referenced to geographic points on the ground. The result combines the image detail of a photograph with the geometric qualities of a map. Orthoimages contain vast amounts of visual information that would be prohibitively expensive to collect by traditional methods.

Orthoimagery could be an important monitoring activity at the lake, which allows managers to see historic conditions, the present state of the lake, and ensures that changes in conditions are recognized and documented. Imagery is used for general mapping, detecting land-cover changes, agricultural uses, zoning applications, property appraisals, feature extraction, and updating other digital datasets. Imagery can be considered to be the primary base-map layer and can contribute to many monitoring activities, except those taking place underground or underwater.

\section{Locations}

Images will be collected over the entire Salton Sea study area, similar to land cover. Compared to certain datasets, like elevation, there are a number of data sources and related projects that can provide imagery, so obtaining this resource over a large study area is feasible. Data can be obtained to observe the most scattered regions either affecting or affected by the lake. Detailed existing imagery is available for Mexico, nearby urban areas, agricultural lands, and mountain watersheds above the lake.

\section{Period and Frequency}

The timing and frequency of collecting orthoimages will be determined on the basis of needs of the other monitoring activities that rely on these images. Imagery over the lake can be obtained anytime through a variety of airborne photography and satellite-imagery vendors.

\section{Protocol and Data Collection}

The number of options for both collecting and locating imagery has increased recently, and thus there are many resources for researchers that can support a very wide variety of studies. Both airborne and satellite platforms can be used to collect imagery. Both have advantages and disadvantages. Airborne images offer higher ground resolutions and are easier to obtain for a given period. Satellite imagery typically offers lower ground resolution, but that is less important because resolutions have improved greatly. The dates of historic satellite data can vary too (that is not able to cover the entire study area on the same day or period,) but custom orders can be placed for future coverage.

The processes and standards for creating aerial orthoimagery are well established. Content standards for digital orthoimagery can be found at http://www.fgdc.gov/standards/projects/FGDCstandards-projects/orthoimagery/orth_299.pdf. The areal extent, ground resolution, spectral bands selected, and level of imagery processing all affect the cost of obtaining the images.

If orthoimagery is to be collected with aircraft, a project area and flight plan will be established. Data content, to include spatial resolution, camera format, bands requests, bit depth, and ancillary products, will be designed to meet the specific objectives of the imaging. The data are acquired, usually 
by using a digital camera. The aircraft also employs airborne GPS and an inertial measurement unit for more precise location determination when the images are taken. The data are fit to ground control points by lining up known points on the earth with the same features on the photograph. The data are orthorectified to remove distortion by fitting the imagery to a terrain model of the region covered. The dataset is then georeferenced to the specified coordinated system and reprojected.

Data Analysis

Orthoimages can be used many ways in monitoring. One of the simplest ways the images are employed is as a backdrop for other datasets. By using the orthoimage as a reference, other data and features being analyzed can be overlain on the image to show their proper location.

Orthoimages serve another use as a source for feature updates. For example, roads and surface water features can be updated simply by overlaying the features over the images and digitizing the changes. The positional accuracy of the imagery is important for this, and it needs to be equal to or better than that of the features being corrected.

Orthoimagery is especially useful in classifying ground cover into either naturally occurring or pre-defined classes. Algorithms are employed to group individual pixels into classes on the basis of their reflectance values and relationship with neighboring pixels. This is the basis for the various landcover/use datasets. More detailed image processing analysis can show vegetative health and highlight areas of land- cover change.

\section{Quality Assurance}

Quality assurance is performed by the vendor supplying the data and then followed with a review by the customer, a third party reviewer, or both. Like the production methodologies, QA processes are well established. First, content and extent of the image tiles are checked. This ensures the entire project area is covered, all files can be read, and data are in the right projection and coordinate system. Next, the positional accuracy of the dataset is checked. Images are accepted by using a tolerance based on image resolution and scale (for example, 1-meter images must support mapping at a 1:12,000 scale or $+/-10$ meters accuracy). Finally, the quality of the image appearance is checked. This includes looking for good color balance between tiles, locating smears and warped features from elevation changes, offset between tiles, sun glint, specular reflection, and cloud cover.

\section{Data Reporting}

Images will be archived and made accessible from the CDFW's BIOS database when appropriate. Images submitted to the CDFW must be provided in a format suitable for inclusion in the BIOS database (see the "Data Management" section), including appropriate metadata.

\section{Overview of Similar Current Monitoring Activities}

The Salton Sea area is well covered by recent imagery. Orthoimagery for all of Imperial County was collected in 2008 with ground resolutions of 6 inches and 1 foot. This dataset also included the Salton Sea shoreline in Riverside County. Caltrans and the Southern California Association of Governments sponsored the data collection. Imperial County was flown again in 2011 by Southern California Association of Governments, with a mixture of 6-inch data for the urban and irrigated portions of the County and 1- foot for the rural portion. The 2011 Imperial County imagery was complemented by a project sponsored by Riverside County that developed images along the northern shoreline of the lake. 
The National Agriculture Imagery Program (NAIP) is managed by the USDA. NAIP is scheduled to a 3- year repeat cycle. NAIP data were collected for California in 2009, 2010, and again in 2012. NAIP is a four band (adding infrared to blue, green, and red) product and is collected at a 1-meter resolution. The 2012 NAIP data for California will be available in fall 2012.

\section{Geology Monitoring Activities}

\section{Landslides and Slumps}

\section{Purpose and Justification}

The Salton Sea area is subject to frequent seismic events that can trigger landslides and slumps in areas of steep topography. Monitoring is necessary to understand the origin and behavior of mass movements and to predict which events (for example, earthquakes or storms) can trigger displacements. Monitoring can provide an early indication of rapid, catastrophic movements. Lateral spreading, or the separating or rupturing of the ground surface as a result of strong ground shaking, can take place along drainage banks, cliffs, or other areas with steep slopes, where generally loose sediments collapse from lack of lateral support. Monitoring in these areas could facilitate a better understanding of slope movement in a particular geographic area as a result of a particular triggering event or over an extended period. Monitoring the movement, crack development, and other physical and temporal aspects of a particular mass movement can lead to a better understanding of the dynamics and, usually, to the development of appropriate stabilization techniques or countermeasures. Monitoring information will also be used to map areas of potential problems so that future construction of facilities on or near these areas is not being adversely affected.

\section{Locations}

Monitoring of landslides, slumps, and lateral spreading will be performed in areas where these processes are likely to happen, such as in steep terrain. Areas prone to landslides can often be recognized through a review of topographic maps geologic and engineering reports, aerial photography and other forms of aerial images, and preliminary field reconnaissance surveys. Landslides and slumps mostly take place in areas of and adjacent to steep slopes often in the mountainous areas well away from the Salton Sea (not the focus of this plan). In the Salton Sea area, there is a potential for slumping on a minor scale along embankments of canals and toe areas of water-impoundment dikes. Within the region, lateral spreading is most likely along the steep channel slopes of the New and Alamo Rivers.

\section{Period and Frequency}

Depending on the severity and consequence of failure, landslides can be monitored on a continuous or periodic basis. The potential for mass movement throughout the area is considered low because of the broad, low-lying character of the land. Hence, it is not anticipated that there will be a need for frequent or real-time monitoring. Monitoring of landslides, slumps, and lateral spreading will take place whenever there is a triggering event, for example, an earthquake. Another opportunity to monitor is after the acquisition of aerial photography and images, which tends to occur every few years.

\section{Protocol and Data Collection}

For purposes of this MAP, landslide monitoring refers to detection of landslides rather than monitoring of active landslides. Landslide monitoring focuses on identifying areas of instability and assessing their state of activity. Data for detection of landslides can be assessed by using maps or remote 
sensing techniques. Maps depicting topography, geology, agricultural soils, and other special terrain and cultural features are generally available in the region. Remote sensing is the collecting of information about an object by the use of sensing devices not in physical contact with the subject under investigation. In this case, remote sensing refers to the use of aerial photography.

Data Analysis

Aerial photography is commonly used in landside monitoring and investigations because it presents an overall perspective of a large area. Aerial photography can also be used to classify land cover. Occasionally, satellite images have been used to investigate and detect landslides. The interpretation of aerial photography has proven to be an effective technique for recognizing and delineating landslides. No other technique can provide a three-dimensional overview of the terrain from which the interrelations of topography, drainage, surface cover, geologic materials, and human activities on the landscape can be viewed and evaluated.

\section{Quality Assurance}

Methodology for landslide monitoring will follow the practices and methodologies cited in Highland and Bobrowsky (2008), The Landslide Handbook--A Guide to Understanding Landslides. The USGS Landslide Hazards Program performs research in order to make accurate landslide hazard maps and forecasts of landslides.

\section{Data Reporting}

Data used to monitor landslides and slumps will be archived and made accessible from the CDFW's BIOS database when appropriate. Data submitted to the CDFW must be provided in a format suitable for inclusion in the BIOS database (see the "Data Management" section), including appropriate metadata.

\section{Overview of Similar Current Monitoring Activities}

Landslide hazard maps, geologic maps, and engineering reports generally are available in the region. Aerial photography is usually taken every 3 years at the Salton Sea. Recent photographs can be compared with older ones to examine the progressive development of slides and slumps.

\section{Sedimentation Rates}

This section focuses on sedimentation in the water environment. Eolian sedimentation is referenced in the Air Quality Section.

Purpose and Justification

Sedimentation refers to the accumulation of solid materials settling out of suspension in a water environment. Sedimentation results from material carried into the Salton Sea through normal surface runoff, flood events, or wind. Knowing the rate of sedimentation and where it takes place in the Salton Sea is very important because sedimentation affects aquatic habitat, accumulation of pollutants, and recreational activities such as boating and fishing.

Monitoring of sedimentation rates is needed to assess the effect of sedimentation (or deposition) and erosion rates in the Salton Sea region and shoreline areas on aquatic habitats, accumulation of pollutants, and recreational activities and to determine if sedimentation rates change as a result of restoration. This information is needed to establish current sedimentation rates and study how and where 
the rates change as the Salton Sea restoration plan proceeds. Sedimentation is related to bathymetry because it alters the lake bed and affects water depth. Rates of sediment accumulation can affect bathymetric data collection frequency and location.

\section{Locations}

Sediment data will be collected from many areas within the bed of the Salton Sea, and the selection of monitoring locations will include consideration of prior sedimentation studies and locations potentially affected by future restoration activities. This wide distribution of collection sites allows for a good understanding of sediment deposition throughout the lake. In addition, sedimentation monitoring will be performed in areas of critical habitat, near water inlets, and in areas of varying currents.

\section{Period and Frequency}

Sedimentation rates can vary with low and high water cycles and natural (floods) and manmade processes (restoration construction). As a result, sedimentation monitoring will be performed seasonally at selected locations for a number of years, as well as before and after specific events that can affect sedimentation rates, such as major storms, algal blooms, earthquakes, or dredging operations. Sedimentation monitoring will be performed at regular intervals, for instance, monthly or quarterly; however, additional monitoring will be performed after specific events or construction and restoration activities. Any studies that have collected information on Salton Sea sedimentation will be used in determining the location or frequency of new sedimentation-rate monitoring.

\section{Protocol and Data Collection}

There are a number of methods for collecting sedimentation data; two of the most common, sediment traps and coring, are needed for monitoring associated with this plan. Sediment traps use a container secured to the lake bed that accumulates sediment over time, which can be measured to determine a rate. Measurements can be expressed as depths (for example $\mathrm{mm} / \mathrm{yr}$ ) or material weight

(grams $/ \mathrm{cm}^{2}$ ). Sediment traps reveal how much sediment is deposited but not when. Sediment traps only measure rates of current sedimentation; they do not tell long-term or historical sedimentation rates unless traps are employed over a long time. Regular observation intervals can reduce uncertainty concerning historical variation. Cores are obtained by drilling into the lake bed and extracting a vertical column of lake bed material, which can be analyzed to determine deposition rates over time. Sediment cores are better suited for determining historical sedimentation rates. Scour chains can be used to measure accumulation of gravels, but this is better suited to regions of variable and high rates of water flow. Ground or aerial-based Lidar can be used in selected locations to survey regions along the shoreline to establish existing conditions from which to assess rates of wind erosion and deposition. For additional information on Lidar, please refer to the Land Surface Elevation monitoring activity described earlier in this chapter.

\section{Data Analysis}

Sedimentation rates will be used to assess how the water column is responding to a number of influences, both natural and manmade. Rainfall, runoff, and underwater or shoreline landslides and slumps are some natural events affecting sedimentation rates. Construction, development, land removed from agricultural uses, and changes in water amounts used in irrigation are some of the manmade causes. Measuring sedimentation rates in conjunction with outside factors will be used to assess how these processes each could affect the lake. The level of detail that sedimentation rates provide depends on the density and frequency of data collection. 
Sedimentation rates in some locations can be used to assess changes in the lake, which could affect selection and or operation of areas for "Species Conservation Habitat." This could be a critical monitoring activity to determine the timing of exposure of lake-bottom sediments in currently shallow areas.

Sediments can be dated by analyzing cores. Cesium-137 can be used to date materials back to about 1950, and Lead-210 can be used for dating sediment deposited since the lake was formed in the early 1900s. Core samples collected for sedimentation rate analysis can also be tested for sediment properties and characteristics, selenium concentration, and other pollutants. The collection and analyses of cores and constituents in cores is dependent on priorities and availability of funds.

Quality Assurance

Guidelines published in the National Institute of Standards and Technology, Standard Reference Materials (SRM) 4354 Freshwater Lake Sediment will be followed during collection of sedimentation data.

\section{Data Reporting}

Data will be archived and made accessible from the CDFW's BIOS database when appropriate. Data submitted to the CDFW must be provided in a format suitable for inclusion in the BIOS database (see the "Data Management" section), including appropriate metadata. In addition, an annual progress report will be prepared that summarizes the data collected during that year and updates prior reports in a cumulative fashion. At a minimum, the annual report is to include the following:

- A summary of data collected during the year.

- Identification of areas with substantial erosion/deposition.

- Assessment of significant changes in chemical composition of sediments.

- Recommendations for improvement of the monitoring protocol, if any.

\section{Overview of Similar Current Monitoring Activities}

No similar sedimentation data collection programs are underway in the region. However, there are a number of previous studies that have collected information on Salton Sea sedimentation. The recent Bureau of Reclamation publication, Restoration of the Salton Sea Final Report, released in 2007 contains sedimentation information in volume 1, appendix 1E. Vogl and Henry (2002) performed a comprehensive study to evaluate the distribution of sediment types and sediment contaminants throughout the Salton Sea. Other research groups that have collected sediment data include Eccles (1979), Levine-Frick (1999), and Anderson and others (2008).

\section{Ongoing Seismic Monitoring}

\section{Purpose and Justification}

Geophysical surveys in and around the Salton Sea are helping to identify the location and geometry of faults in the area. The existing seismic network in southern California (http://www.cisn.org/instr/) monitors earthquakes and archives and distributes information for improved public safety, emergency response, loss mitigation, and scientific research. The instrumentation consists 
of southern California seismic network sensors, national strong motion program sensors, and California Geological Survey strong motion sensors. Given the proximity of the Salton Sea to the southern extent of the San Andreas Fault, a number of seismic stations already are established in the Coachella and Imperial Valleys. There is a need to continue to gather earthquake data, such as the magnitude, location, and damage from the event, to coordinate rescue operations and to expand scientific research. The historical record of earthquakes in California is important to seismological researchers, insurers, geotechnical engineers, and city planners. One of the primary goals is to implement lessons learned from earthquake data and to incorporate them into seismic code provisions and seismic design practices. The MAP will provide the mechanism for continued seismic monitoring in the Salton Sea region.

\section{Locations}

There are currently a few seismic monitors located around the perimeter area of the lake. New seismic monitors could be proposed at selected locations after analyzing the results of ongoing field work, and these would be incorporated into the overall seismic network. In addition, given potential power requirements associated with seismic network stations, the potential for collocation with air quality and other data needs would be examined.

\section{Period and Frequency}

Seismic monitoring is performed on a continuous, real-time basis and will be performed for the duration of the MAP.

\section{Protocol and Data Collection}

The Standard for the Exchange of Earthquake Data (SEED) is an international standard format for the exchange of digital seismological data. SEED was designed for use by the earthquake research community, primarily for the exchange between institutions of unprocessed earth-motion data. It is a format for digital data measured at single points in space at equal intervals of time. SEED helps seismologists who record, share, and use seismological data. By providing a standard, SEED makes transmitting, receiving, and processing earthquake data easier and more accurate than it was before.

\section{Data Analysis}

The primary source of data is a network of seismic sensors placed in the Salton Sea region. Generally, there are shared agreements among the various organizations collecting the data to enable the sharing of data. Data from the monitoring devices are retrieved by modem and computers or by physically recovering the records at the station. Following an earthquake, records are collected, processed, and disseminated to engineers, seismologists, building officials, state and local governments, and emergency personnel throughout the State. When activated by earthquake shaking, the devices produce a record from which important characteristics of ground motion (acceleration, velocity, displacement, and duration) can be calculated. These parameters are used for various applications in engineering analyses.

\section{Quality Assurance}

All data collected will follow the standard format for the exchange of earthquake data (SEED) as described previously under data collection. Quality-assurance plans for existing data collection efforts by the USGS and California Institute of Technology associated with earthquake data in the area will be assessed, revised if needed, and followed during implementation of the MAP. 


\section{Data Reporting}

All data collected will be reported to the California Geological Survey following the standard format for the exchange of earthquake data (SEED) as described previously under data collection. Each year that monitoring is performed, data will be submitted to and made accessible from the CDFW's BIOS database. Data submitted to the CDFW must be provided in a format suitable for inclusion in the BIOS database (see the "Data Management" section), including appropriate metadata. An annual progress report will be prepared that summarizes the data collected during that year and updates prior reports in a cumulative fashion. At a minimum, the annual report is to include the following:

- A summary of data collected during the year.

- An assessment of effects of earthquakes on the ecosystem, if any were specifically documented.

- A reference to publications on earthquakes in the Salton Sea Ecosystem, if any.

- Recommendations for improvement of the monitoring protocol, if any.

Overview of Similar Current Monitoring Activities

The existing seismic network consists of instrumentation from the southern California seismic network sensors, national strong motion program sensors, and California Geological Survey strong motion sensors. There is a partnership among federal, state, and university agencies involved in California earthquake monitoring. The partnership is dedicated to serve the emergency response, engineering, and scientific communities. In addition, there are various other types of monitoring on a regional and local basis, such as geodetic instruments to measure the slow movement of the earth's crust and strain meters on the San Andreas Fault to measure the accumulating strain. 


\section{Air-Quality Monitoring}

\section{Overview}

This air-quality monitoring plan focuses on activities designed to characterize existing (or background) and longer-term air quality and meteorological conditions at the Salton Sea. This plan describes monitoring for 1) ambient concentrations of criteria pollutants and other pollutants of interest, and 2) surface meteorological conditions.

Air quality in the Salton Sea Air Basin does not currently achieve California or National Ambient Air Quality Standards (CAAQS or NAAQS) for PM10 (particulate matter that will pass through a 10 micron filter) and ozone, although the EPA recently found the Imperial County portion of the Salton Sea Air Basin to be in attainment of the NAAQS for ozone (http://www.gpo.gov/fdsys/pkg/FR-2009-12-03/pdf/E9-28536.pdf) (Federal Register, 2009). In general, the pollutants of greatest concern in the area are 1) particulate matter (PM10 and PM2.5) from wind erosion (fugitive dust), soil disturbance, and fuel combustion; and 2) ozone and the ozone precursors, nitrogen oxides (NOx), and volatile organic compounds (VOCs), primarily from vehicle and equipment exhaust. Agricultural operations and transport of pollutants from Mexico also contribute to air-quality issues in the area.

Environmental documents prepared for the Imperial Irrigation District Water Conservation and Transfer Project and the Salton Sea Ecosystem Restoration Program predicted that potentially significant effects to air quality due to fugitive dust from wind erosion of exposed lakebed playa as water levels in the Salton Sea are reduced (Imperial Irrigation District and U.S. Bureau of Reclamation, 2002; California Resources Agency, 2006). In addition, air-quality effects can result from construction and operation of ecosystem restoration program elements, as a result of equipment exhaust and fugitive dust emissions. Ecosystem restoration activities could also affect the levels of hydrogen sulfide released from geothermic and biogenic sources in the Salton Sea.

Monitoring is needed to establish existing conditions for ambient air quality at and near the Salton Sea. Monitoring data can serve as a baseline for observation of longer-term incremental changes in pollutant concentrations and support future evaluations of air-quality management and mitigation efforts.

\section{Objectives}

The objectives of the air-quality and meteorological monitoring plan fall into two general categories: 1) those related to establishment of existing (or background) conditions and documentation of longer term conditions that could change over time (trends), and 2) the identification, prioritization, and filling of data gaps that relate to Salton Sea air quality and meteorological conditions. Additional information is needed to characterize existing air-quality conditions and emission sources at the Salton Sea, and to support estimation of potential future emissive conditions and planning of air qualitymanagement actions. Information is also needed to identify and better understand the relationships among emission sources, pollutants, meteorological factors, and other causes of variability in air quality. Results from the ambient air-quality and meteorological monitoring program also could be used to support ongoing assessment of air-quality effects related to the Imperial Irrigation District Water Transfer Project and ecosystem restoration at the Salton Sea. 


\section{Key Questions and Information Needs}

Key questions to be addressed by the air-quality monitoring plan were identified through collaboration with the Air Quality Focused Technical Working Group, a group of experts convened for the purpose of providing input to the monitoring and assessment requirements for Salton Sea air-quality resources. Key questions were identified in the areas of ambient pollutant concentrations, meteorological conditions, chemical composition of windblown dust, particle deposition rates, and playa characteristics and potential emissions. The full list of key questions identified by the focused technical group is included in appendix 7.

The key questions were evaluated to identify the primary issues and then reviewed to determine whether the question could be answered through monitoring (that is, repeated measurements over time) or through focused investigations (including literature review or assessment of existing information). For those issues that could be answered in whole or in part by monitoring activities, the specific monitoring activities to collect the information needed to answer or contribute to the answers of key questions were identified. The specific information needs associated with answering the key questions and the monitoring activities needed to collect that information are presented in table 15 . The specific monitoring activities associated with those key questions are described in greater detail in subsequent sections of this monitoring plan.

\section{Focused Investigations}

In addition to the monitoring programs for ambient air-pollutant concentrations and surface meteorological conditions outlined in this plan, focused investigations are needed to clarify key questions and provide needed information regarding existing and future air-quality conditions at the Salton Sea. While these investigations will not be planned, designed, and implemented under this monitoring plan, they play an important role in clarifying the processes that contribute to air quality and informing decision making at the Salton Sea. In some cases, focused investigations, as stand-alone studies, can be sufficient to answer specific key questions. In other cases, focused studies could identify the need for longer-term monitoring. For example, depending on outcomes, initial research on the chemical composition of particulate matter, particle deposition, or shoreline microclimatic conditions, performed under existing conditions, could lead to longer-term monitoring and development of additional monitoring plans.

As another example, focused investigations could increase the current level of knowledge of Salton Sea playa characteristics, such as dust emission potential, particulate matter emission rates for disturbed and undisturbed playa, threshold wind velocities for dust generation, salt crust genesis, chemical composition, physical characteristics, and how these factors vary in relation to time exposed, distance from the shoreline, depth to groundwater, and the presence of sand. Knowledge of Salton Sea playa gained from these initial focused studies can help researchers identify what longer-term playa monitoring programs are needed and what parameters are important to be monitored as changes take place.

Initial focused investigations can be used to 1) directly answer some key questions, 2) characterize key questions that will require monitoring programs for answers, 3) clarify monitoring objectives and approaches, and 4) provide information critical to the development of programs to meet those objectives. At the Salton Sea, key questions indicate the need for other focused investigations, such as the following: 
- Measurements of upper air meteorological conditions.

- Use of remote sensing and satellite imagery to track changes in exposed Salton Sea shoreline areas.

- Back-trajectory analysis to predict the sources of monitored particulate matter.

- Development and pilot testing of a "toolbox" of possible dust control measures.

- Investigations of potential odorous emissions.

- Identification of needed tools and models to support future studies.

- Estimation of greenhouse gas emissions from restoration activities.

- Evaluation of potential effects of global climate change on the Salton Sea and the Salton Sea Air Basin.

\section{Geographic Scope}

The geographic scope for the assessment of air quality is the Salton Sea watershed. This area roughly corresponds to the Salton Sea Air Basin, which is composed of Imperial County and the Coachella Valley portion of Riverside County (fig. 12). For monitoring of existing or background airquality and meteorological conditions, the geographic scope is the near-shore area surrounding the Salton Sea. Longer-term air-quality monitoring could address larger, smaller, or differing geographic areas, depending on the monitoring objectives.

\section{Conceptual Model}

The key questions in appendix 7 were developed in part by using a conceptual model for airquality resources at the Salton Sea (fig. 13). The conceptual model provides a relatively simple depiction of the complex relationships among inputs to and outputs from the ambient air-quality system (for example, weather, pollutants, sources, and receptors) and the processes that drive change. This conceptual model serves as a useful tool for refining the understanding of how the system functions, where there are data gaps, and where additional data collection is needed.

\section{Air Quality Monitoring Activities}

Air-quality monitoring is currently needed near the Salton Sea to characterize existing air-quality conditions and background (existing) emission sources and to support evaluation and management of longer-term conditions and sources. For example, baseline data on ambient PM10 concentrations will be collected prior to exposure of lakebed playa, or ecosystem restoration program activities, to support the evaluation and mitigation of PM10 emissions and effects resulting from playa- and restoration-related sources. This monitoring activity will be performed as soon as possible, ideally before conditions at the Salton Sea begin to change.

As Salton Sea ecosystem restoration projects are designed and implemented, air-quality monitoring will be performed during each project phase. During the initial monitoring and design phase, ambient monitoring of particulate matter (mass and species), gaseous criteria pollutants, and toxic pollutants naturally occurring at the lake will be performed to characterize existing or background levels 
of these pollutants in the absence of playa- or restoration-related emission sources. The data from this monitoring phase will also support design of the monitoring networks for the construction and operational phases. Results can also be used in the development of technical tools, such as an emission inventory and air-quality models. The specific objectives of the air-quality monitoring activities and their intended purposes are presented in table 16.

\section{Ambient Air-Quality Monitoring}

\section{Purpose and Justification}

As Salton Sea water levels drop, and ecosystem restoration projects and the related emissions take place, ambient air pollutant concentrations measured near the Salton Sea will include emission contributions from playa- and restoration-related sources, as well as ongoing contributions from background (non-Salton Sea) sources. Management decisions require knowledge of existing and longerterm air pollutant concentrations as well as estimates of existing and future emission sources and contributions.

\section{Locations}

Ambient air-quality monitoring can be performed at different locations under different phases of an ecosystem restoration program. During the initial data collection phase, ambient air-quality monitoring is designed to be performed near shoreline, populated areas and in areas conducive to the assessment of contributions from background emission sources that are not related to exposed Salton Sea playa or ecosystem restoration. During the construction and operation of restoration facilities, monitoring will be performed near populated areas and in the areas predicted to be the most affected by emissions from the proposed construction and operation activities.

On the basis of an initial assessment of existing meteorological and onshore source conditions, six locations for monitoring were identified, and preferred specific sites were selected. The primary considerations used in site selection follow:

- Power availability within 100 feet of the proposed site pad.

- Reasonable access by vehicle.

- Site security through mechanisms such as access control, location in a remote area without evidence of substantial human activity, or observation of site by nearby residents.

- Lack of nearby significant land-surface disturbance (and resultant dust emissions) from human activity.

- Cell-phone reception.

Ambient air-quality monitoring equipment has been installed at the sites identified as preferred sites. The preferred monitoring site locations are shown in figure 12, and their descriptions follow. 
Site 1-Salton Sea Naval Test Site

The monitoring site is east of State Highway 86, approximately 16.5 miles northwest of Westmorland, California. The site is on land owned by the Federal Government and managed by Reclamation.

Site 2 - Salton City

This site is at a Salton Community Services District wastewater-treatment plant, which is on a 40-acre tract near the southern end of Salton Drive in Salton City. The site has power, a cyclone fence, no trees or obstructions, and no nearby off-road vehicle use. The site is on property owned by the Salton Community Services District.

\section{Site 3-Whitewater River Outlet, North Shore}

This site is owned by the Torres Martinez Tribe and is adjacent to a wetlands project being developed by the Tribe. The site is bounded by a cyclone fence and waterways, and the Tribe is having a three-phase power line installed underground to the site from an electrical junction 1,600 feet away. A locked gate maintained by wetland project personnel is located at the intersection of Lincoln Avenue and the unpaved access road.

\section{Site 4 -Salton Sea Recreation Area}

The monitoring site is located west of the entrance booth along State Park Road at the Salton Sea Recreation Area headquarters facility and visitor's center. The site is approximately 225 feet from an underground three-phase power line. The site is on property owned by the Federal Government and managed by California State Parks.

\section{Site 5 -Bombay Beach}

The monitoring site is west of the intersection of A Street and $3^{\text {rd }}$ Street in Bombay Beach. The site has three-phase power and is on land owned by the Federal Government and managed by California State Parks. An earthen berm and ditch along the west side of A Street appears to prevent access by offroad vehicles to the area surrounding the site.

Site 6-Salton Sea South Shore

This site is on the premises of the Sonny Bono Wildlife Refuge at the intersection of Boyle Road and Sinclair Road on the south shore of the Salton Sea. The site has three-phase power and is a minimum of 600 feet from active agricultural operations. The site is on land owned by the Imperial Irrigation District and leased to the U.S. Fish and Wildlife Service.

\section{Period and Frequency}

Particulate matter concentrations will be monitored continuously and reported as hourly averages. The hourly values will be computed by the data loggers at each monitoring station and transmitted shortly after the end of each hour to a central server operated by the Imperial County Air Pollution Control District (ICAPCD). The format for onsite storage of hourly average data will enable hourly polling by the central server and retransmission to the California Air Resources Board (ARB) for online real-time display.

Continuously monitored particulate-matter concentrations will also be cached in 5-minute averages in the data loggers at each monitoring station and uploaded manually at the end of each month 
to the central server operated by ICAPCD. After an initial quality-control check, the sub-hourly data files will be manually forwarded to ARB for storage.

Gas monitors are recommended for future monitoring and will be operated on a continuous basis and programmed to report hourly average values. The monitored gaseous pollutants will include ozone, oxides of nitrogen (NOx), sulfur dioxide $\left(\mathrm{SO}_{2}\right)$, carbon monoxide $(\mathrm{CO})$, hydrogen sulfide $\left(\mathrm{H}_{2} \mathrm{~S}\right)$, and ammonia (NH3). Two monitors for each pollutant will be rotated annually among the six monitoring stations.

As Salton Sea ecosystem restoration projects are designed and implemented, air-quality monitoring will be performed during each project phase.

\section{Protocol and Data Collection}

The collection and analysis of air pollutant samples will be performed in conformance with operating and analysis protocols approved by ARB. Assessment of data quality will be continuously evaluated through compliance with the protocols of the quality-assurance project plan (QAPP) developed and implemented by the ICAPCD.

\section{Data Analysis}

Particulate Matter (PM)coarse $\left(P M_{10-2.5}\right)$ and $P M_{2.5}$

PMcoarse $\left(\mathrm{PM}_{10-2.5}\right)$ and $\mathrm{PM}_{2.5}$ (please see http://www.arb.ca.gov/html/brochure/pm10.htm for definitions) will be both continuously and episodically collected at each of the six monitoring stations. Continuously monitored data will be averaged over 5-minute and 1-hour periods and forwarded to ICAPCD for initial quality-assurance review and then to ARB. Hourly PM data will be loaded onto the ARB real-time monitoring data website (http://www.arb.ca.gov/aqmis2/aqinfo.php) within 20 minutes following the end of each hour. After validation, these data will be used by air-quality agency staff, researchers, and the general public to assess information on existing PM levels around the Salton Sea.

Five-minute average PMcoarse $\left(\mathrm{PM}_{10-2.5}\right)$ and $\mathrm{PM}_{2.5}$ data will be uploaded monthly from the monitoring station data loggers to the ICAPCD central server, where an initial quality-control review will be performed. These files will then be forwarded to ARB for placement on an FTP site for access by Salton Sea researchers.

PMcoarse ( $\left.\mathrm{PM}_{10-2.5}\right)$ and $\mathrm{PM}_{2.5}$ samples will also be collected on filters over 24-hour averaging periods for speciation analysis and a quality-assurance check of the continuous PM monitoring data.

Two deposition monitors are recommended for future monitoring and will be rotated among collection sites every year. Deposition monitors will be used at the monitoring stations and at sites remote from monitoring stations to quantify particulate matter deposition rates near the Salton Sea shoreline. The deposited particulate matter will be collected from the monitors at the end of each month and analyzed for mass and elemental composition.

\section{Ozone and NOx}

Ozone and nitrogen oxides (NOx) will be monitored continuously and reported in 1-hour averages to ICAPCD and ARB. Two monitors are recommended for each pollutant, and these monitors will be rotated annually among the six monitoring stations. The pollutant concentration data collected by these monitors will be integrated with data from the existing Coachella and Imperial Valley stations to improve geographic coverage of the area and quantification of the levels of these pollutants near the Salton Sea. 
The Salton Sea Ecosystem Restoration Program Draft Programmatic Environmental Impact Report (California Resources Agency, 2006) estimated that earthmoving and support equipment planned for use in constructing the alternative closest in design to the "Preferred Alternative" will produce annual NOx emissions during the peak construction year that are equal to about 20 percent of the Imperial County annual NOx inventory. If future ecosystem restoration moves forward in a manner similar to that identified in the Preferred Alternative, the resultant NOx emissions could measurably increase ozone concentrations downwind of the Salton Sea. Measurement of ozone, NOx, and meteorological conditions during the initial monitoring period will facilitate a more accurate analysis of the effects on local ozone levels from construction NOx emissions and support the analysis of needed mitigation.

\section{Sulfur Dioxide, Hydrogen Sulfide, and Ammonia}

Carbon dioxide $\left(\mathrm{CO}_{2}\right)$ Sulfur dioxide $\left(\mathrm{SO}_{2}\right)$, hydrogen sulfide $\left(\mathrm{H}_{2} \mathrm{~S}\right)$, and ammonia $\left(\mathrm{NH}_{3}\right)$ are generated in the Salton Sea by geothermal activity and anaerobic decomposition. A number of tectonic faults cross under the Salton Sea, some of which release gaseous compounds, primarily $\mathrm{H}_{2} \mathrm{~S}_{\text {and }} \mathrm{CO}_{2}$. During periods of reduced vertical mixing in the Salton Sea, $\mathrm{H}_{2} \mathrm{~S}$ tends to accumulate in the deeper, cooler areas of the lake. The area below the thermocline tends to be devoid of oxygen (anoxia), and, in the absence of oxygen, bacteria will use sulfate as the terminal electron acceptor during the decomposition of organic matter. When an upwelling of the lake is caused by a high wind event, the mixing of stratified water releases $\mathrm{H}_{2} \mathrm{~S}$ into the water column and the atmosphere. Upon release, $\mathrm{H}_{2} \mathrm{~S}$ combines with dissolved oxygen and calcium, sulfide is oxidized to sulfate, and large fish kills can result from anoxia.

Two continuous monitors are recommended for each pollutant $\left(\mathrm{SO}_{2}, \mathrm{H}_{2} \mathrm{~S}\right.$, and $\left.\mathrm{NH}_{3}\right)$, and these monitors will be operated for annual periods at each monitoring station on a rotating basis. Data collected will be used to assess changes in $\mathrm{SO}_{2}, \mathrm{H}_{2} \mathrm{~S}$, and $\mathrm{NH}_{3}$ emissions during the construction and operation phases of an ecosystem restoration program.

\section{Quality Assurance}

Air-quality and meteorological monitoring will be performed in conformance with the protocols of a QAPP developed and implemented by the ICAPCD (appendix 8).

\section{Data Reporting}

Pollutant concentrations, with the exception of some particulate-matter samples, will be monitored continuously and reported as hourly averages. The hourly values will be computed by the data loggers at each monitoring station and transmitted shortly after the end of each hour to a central server operated by the ICAPCD. The format for onsite storage of hourly average data will be consistent with formats now in use by ICAPCD at existing monitoring stations. This format enables hourly polling by the central server and retransmission to the ARB for online real-time display.

Continuously monitored particulate-matter concentrations will also be cached in 5-minute averages in the data loggers at each monitoring station and uploaded manually at the end of each month to the central server operated by ICAPCD. After an initial quality-control check, the sub-hourly data files will be manually forwarded to ARB for storage. Access to the data FTP site will be made available to researchers on a request-basis. These data will not be formatted or uploaded to ARB's real-time display system. 


\section{Overview of Similar Current Monitoring Activities}

Air-quality and meteorological monitoring is currently performed in areas near the Salton Sea by the ICAPCD, the South Coast Air Quality Management District, the Torres Martinez Tribe, and ARB. A list of regional monitoring stations and the parameters they monitor is shown in table 17. See figure 12 for a map of these monitoring stations. Data collected by existing regional monitoring stations will be integrated with data collected by the Salton Sea air-quality monitoring network to evaluate geographic and temporal trends.

\section{Surface Meteorological Monitoring}

\section{Purpose and Justification}

Surface meteorological monitoring is needed to evaluate eolian sedimentation and pollutant transport and production mechanisms in the Salton Sea area. Meteorological monitoring will be used in conjunction with ambient air pollutant concentration monitoring to evaluate pollutant transport from existing emission sources within and upwind of the Salton Sea Air Basin. The meteorological data will also be used to populate emission dispersion models for forecasting cumulative downwind pollutant gradients during periods of pollutant-generating activities related to ecosystem restoration. The potential utility of sand-motion monitoring and video analysis to identify the location of emissive areas on the playa will be assessed. Finally, wind-speed data support predictive models used to evaluate potential windblown-dust emissions from exposed or disturbed lakebed playa.

Local wind-speed data are needed to estimate the $\mathrm{PM}_{10}$ emission rates of exposed playa under high wind speed conditions. Because of the power relationship between wind speed and emissions, and because the short-term variability in wind speed during high wind events, the accuracy of emission estimations are dependent on the monitoring of wind speed over very short periods, which requires continuous monitoring and frequent storage of wind-speed data.

Locations

Surface meteorological data will be collected at the same sites used to continuously monitor concentrations of PMcoarse $\left(\mathrm{PM}_{10-2.5}\right)$ and $\mathrm{PM}_{2.5}$. These sites are described previously in the data analysis section.

\section{Period and Frequency}

Surface meteorological data will be continuously monitored. One-hour average values will be computed by the data logger at each monitoring station and reported to the publically accessible ARB AQMIS system within 20 minutes after the end of each clock hour of monitoring. Five-minute average values will be computed by each data logger and stored in batch files for monthly uploading to an ARB FTP site. These batch files will be available for any researcher on a request basis.

\section{Protocol and Data Collection}

Surface meteorological instruments will be operated in conformance with a QAPP developed and implemented by the ICAPCD.

\section{Data Analysis}

The following data will be directly monitored by the meteorological instruments at each of the air-quality monitoring stations: 
- Wind direction (U, V, and $\mathrm{W}$ vectors at the 10-meter height on each meteorological tower).

- Wind speed.

- Two- and three-dimensional at the 10-meter height on each meteorological tower by using a sonic anemometer.

- Scalar at the 1-meter, 2-meter, and 10-meter heights on each meteorological tower by using cup anemometers.

- Temperature.

- At the 3-meter inlet to each Tapered Element Oscillating Microbalance (TEOM).

- At the 10-meter height on each meteorological tower.

- At the 2-meter height on each meteorological tower.

- Relative humidity (at the 3-meter inlet to each TEOM sampler and at the 2-meter height on each meteorological tower).

- $\quad$ Barometric pressure (at the 3-meter inlet to each TEOM sampler).

- Solar radiation (at the 1-meter height on each meteorological tower).

Instruments to measure precipitation and evaporation rates will also be installed if these data are needed to support focused investigations. From the monitoring data, the following meteorological parameters will be calculated by each station data logger and stored:

- Wind direction (scalar and vector at the 10-meter height on each meteorological tower)

- Wind speed (2-dimensional and 3-dimensional scalar and vector at the 10-meter height on each meteorological tower)

- Sigma theta (standard deviation of 2-dimensional and 3-dimensional wind directions at the 10meter height on each meteorological tower)

- Delta-T (temperature difference between 2-meter and 10-meter heights on each meteorological tower)

Quality Assurance

Surface meteorological instruments will be calibrated and maintained in conformance with a QAPP developed and implemented by the ICAPCD.

Data Reporting

The meteorological data collected and calculated at each monitoring station will be used primarily to generate meteorological databases needed to run statistical, Gaussian, and puff air pollutant 
dispersion models. Results of modeling and other related studies will be documented in reports and posted on the ARB FTP web site.

Overview of Similar Current Monitoring Activities

Air quality and meteorological monitoring is currently performed in areas near the Salton Sea by the ICAPCD, the South Coast Air Quality Management District, the Torres Martinez Tribe, and ARB. A list of regional monitoring stations and the parameters they monitor is shown in table 17. See figure 12 for a map of these monitoring stations. Data collected by existing regional monitoring stations will be integrated with data collected by the Salton Sea air-quality monitoring network to evaluate geographic and temporal trends. 


\section{Socioeconomic Monitoring}

\section{Overview}

This section highlights important socioeconomic factors to be monitored on an ongoing basis as part of the Salton Sea monitoring and assessment plan. The history of human settlement and socioeconomic activity around the Salton Sea dates back to early Native American settlements and is diverse and unique. A common thread throughout all socioeconomic activity is dependence on the environment of the Salton Sea and the surrounding region, and how changing conditions have directly affected individuals, communities, and social systems within the Salton Sea region, especially those nearest the lake.

Throughout its history, the Salton Sea has experienced constantly changing physical, biological, social, and economic conditions. Long-term socioeconomic monitoring could provide a means by which changes in social, cultural, and economic conditions associated with restoration efforts can be detected and documented over time. Restoration of the Salton Sea is not only intended as a means to improve the physical and biological conditions of the Salton Sea region, but would also consider effects on socioeconomic systems reliant on the lake. As the environmental context of the Salton Sea is changed through restoration efforts, there could be effects on the sociodemographic and economic status and dynamics of the community. Restoration efforts could introduce new opportunities and challenges for communities tied to the Salton Sea ecosystem.

Today, economic activity in the region includes agriculture, recreation, tourism, fisheries, real estate, and geothermal energy. A variety of communities rely on the Salton Sea in different ways; however, the common link among all uses is their connection to the lake. There are also a variety of stakeholders who live outside the immediate basin who derive benefits from the lake (for example, groups and organizations associated with bird watching and the Pacific Flyway). The Salton Sea is critically located in the Pacific Flyway, and any disruption to this flyway path could negatively affect migratory birds and, in turn, disrupt industries associated with bird watching — both locally and in other regions. Furthermore, given the high price of housing in southern California, residential communities around the lake have become an important option for affordable housing.

As the water level and water quality of the lake change, so do the social, demographic, and economic conditions. For example, some tourist-based uses were founded because of resource availability (fish) from the lake. As the presence or absence of sport fish has changed, so have the uses. Similarly, as the water quality, salinity, and level have changed, so have the human uses and economic benefits provided by the lake. In the 1950s and 1960s, the lake was a tourist and recreation destination for southern Californians that provided opportunities for boating and fishing. Today, because of elevated selenium levels, the State of California advises consumption of no more than two servings per week of fish caught in the lake (Office of Environmental Health Hazard Assessment, 2009), and recreational use of the lake is largely limited to shoreline activities, such as bird watching. This is a significant change from the time of sport fishing, recreation, and boating.

\section{Objectives}

Initially, the objectives of socioeconomic monitoring and assessment are to do the following:

1. Identify existing socioeconomic conditions, including demographic and economic indicators in the region such as, identification of socioeconomic communities, clusters and dependencies. 
2. Locate these socioeconomic groups, their interactions, and dependencies in both geographic space (mapped) and conceptual space (socioeconomic network mapping).

3. Identify and map land uses and evaluate how these relate to socioeconomic groups and locations.

4. Identify socioeconomic benefits provided by the Salton Sea, including outputs (for example, tons of fish or number of visitors), jobs (for example, related to research or outputs), increase in property value, and so on, relative to the distribution of these benefits among the socioeconomic groups.

5. Assess how restoration activities could affect socioeconomic groups.

6. Monitor the trends in demographic and economic indicators as well as the socioeconomic benefits on a consistent basis to learn how they change over time and how these changes relate to the restoration and changing conditions of the Salton Sea.

These objectives reflect the limited information and current understanding of socioeconomic conditions. The objectives will be reassessed following completion of a retrospective analysis in order to assure that the most appropriate comprehensive analyses techniques can be applied. Examining these existing socioeconomic conditions establishes indicators for ongoing collection to provide the requisite data for long-term monitoring and assessment of the Salton Sea 'socioeconomic catchment' or 'basin.' It is essential to determine appropriate existing conditions for the purpose of understanding these changes over time. Because socioeconomic and demographic conditions are associated with value judgments (for example, what is the desired condition as defined by stakeholders?), measured changes in specific parameters can be considered either desirable or undesirable. These judgments are largely independent of the data collection and analysis, but nonetheless are important considerations.

\section{Key Questions and Information Needs}

To address the questions of socioeconomic conditions and effects of restoration activities (including clusters and dependencies), there are a variety of information requirements. Some of these will come from readily available data sources, whereas others will need to be collected specifically for the purpose of socioeconomic monitoring in the region.

The Draft Programmatic Environmental Impact Report (California Resources Agency, 2006) describes a number of datasets and assesses their value and limitations. Data of particular value for socioeconomic analysis are discussed in chapters 12 (population, housing) and 22 (social and economic). Both chapters base their assessment on the same four data sources provided by federal, state, and regional government agencies. These include (1) the United States 2000 Census completed by the U.S. Census Bureau, (2) annual estimates completed by the Demographics Research Unit of the California Department of Finance, (3) reports prepared by the Southern California Association of Governments (SCAG), and (4) reports prepared by the California Employment Development Department (EDD). The greatest level of detail regarding population and housing resources is provided by the Census Bureau.

Limitations of these data are described in the draft PEIR (California Resources Agency, 2006) as follows:

Historical information is reported every 10 years and may not reflect annual fluctuations. Published projections are based on statistical extrapolation of historical data, taking into consideration 
potential changes in economic conditions, household composition, and demographic variables, such as vital statistics and migration rates. Any projections can be affected by numerous variables, such as job growth, availability of affordable financing, and personal desires of existing and future populations.

Current SCAG population projections prepared in 2004 include forecasts to 2030, while the Demographics Research Unit projections extend to 2050. Population projections are not available for the entire 75-year study period.

Socioeconomic clusters can be defined by a composite of data from multiple sources. Many of these data are collected during the decadal United States Census, which can provide a useful context for developing an understanding of the socioeconomic clusters at a regional level. However, the granularity of the U.S. census is not adequate to monitor long-term changes at the level of individual communities and clusters that is necessary to understand how the restoration process affects these clusters. To monitor changes in the region at a level of resolution appropriate to understand changes and rates of change within and among the existing socioeconomic clusters (and to compare these trends to meeting desired outcomes for the region), detailed data regarding the socioeconomic clusters will be collected on a biennial basis. These data will be collected in a manner that is comparable to the U.S. Census, which provides a means for data comparisons and validation on a decadal basis. Additional techniques of analysis, including looking at communities rather than clusters, will be explored following the retrospective analysis and initial phases of monitoring.

Similarly, the Economic Census, which profiles American business, is performed every 5 years. Additionally, the State of California Employment Development Department collects somewhat more detailed data than the Economic Census. While these and other available datasets provide important information, they cannot provide a sufficiently comprehensive picture of the region for several reasons. First, the Economic Census and Employment Development Department data are based on information collected from businesses with employees that are located within a specified region. Because some businesses operate as sole proprietorships, and many residents of the region commute outside the Salton Sea basin for work, a portion of the population is not captured by these data collection efforts. Collection of economic data on businesses operating in the region, including those missed by these other efforts, will be essential in monitoring the effects of restoration on these communities and within and among socioeconomic clusters. These data will be collected in conjunction with other data on a biennial basis.

To develop an understanding of the social networks among clusters, additional information is required to examine relationships between and among various clusters in the region and, to an extent, externally to it. These data can be obtained through a limited number of questions focusing on which groups interact with each other in various contexts, including those categorized as necessary (for example, business, educational, or governmental relationships) and those that are by choice (for example, social relationships). These data will be obtained simultaneously with socioeconomic data collection.

\section{Geographic Scope}

The geographic extent of monitoring activities will include the Salton Sea socioeconomic catchment in conjunction with associated Salton Sea socioeconomic clusters that have an essential relationship with to the lake. (for example, human labor that lives in communities around the lake because the housing is more affordable and commute elsewhere to work where they cannot afford to live such as Palm Springs). Political boundaries that work with economic models (such as zip codes) 
also could be used in the analyses. The socioeconomic catchment is defined as a region that includes spatially and conceptually defined clusters, networks, and patterns of interaction with a directly demonstrable relationship with the Salton Sea.

Sociodemographic patterns and trends are clearly influenced by land use in the region. Therefore, early in the research process, socioeconomic, demographic, and land-use maps for the Salton Sea watershed, and for all related socioeconomic catchments, will be obtained or created from existing data sources.

Because the precise extent of the socioeconomic clusters cannot be determined until data are collected and analyzed for an understanding of clusters and relationships among them, an initial geographic boundary consisting of the Salton Sea watershed will be used, and adaptations can be made as data collection progresses.

For relationships with more far-flung socioeconomic clusters (beyond the immediate socioeconomic catchment), but for which the Salton Sea represents an integral component, data will be obtained from readily available local, state, and federal sources, with supplemental information coming from targeted studies addressing issues of particular interest. Examples include a survey of stakeholders or communities with interests in bird watching along the Pacific Flyway for which the Salton Sea is an essential component, or for which the Salton Sea provides offsetting ecological services (for example, acting as a replacement for degraded coastal marshes along the southern California Coast).

Data relating to land use is typically maintained by county and city governments, and these data are sufficient to track the designated use of parcels in the region. However, land-use designations and actual land use do not always coincide, so it can prove useful to include additional ground-truth checks on a subset of parcels over time. For example, a parcel can be designated as agricultural, but it is important to know what, if any, crops are being grown on that parcel during a particular time-frame. Changes to the overall crop mix, and how restoration affects such changes, can be an important indicator of overall trends for the region, both positive and negative.

\section{Conceptual Model}

Figure 14 depicts the essential components of the Salton Sea economic system with an emphasis on natural resource-related stocks and flows. Changes in characteristics of the lake affect the economic system through the linkages shown in the figure. Therefore, the figure provides guidance for appropriate monitoring of effects on both stocks and flows of resources, where boxes represent stocks, and arrows are flows. Most arrows imply a compensatory flow in the opposite direction (for example, wages paid for labor), although some do not (for example, uncompensated use of amenities and ecosystem services). A brief explanation follows.

Local residents import resources, including Colorado River water, which can either contribute to the stock of non-firm capital (for example, residential real estate or public infrastructure) or can be invested in the stock of private firm capital or natural capital (for example, the lake) through preservation and restoration efforts. Local residents, and perhaps some non-local residents, also contribute labor to both agricultural and non-agricultural firms. Agricultural firms convert labor, investment, and other factors of production, including Colorado River water and ecosystem services provided by the Salton Sea (for example, climate regulation), into firm capital and outputs. Primary outputs to both local and non-local residents are crops and water transfers. Non-agricultural firms do the same; their primary outputs are recreation services (for example, hotels and resorts, boat rentals, and fishing charters), commercial fishing, and other goods and services, including those of the local secondary economy (for example, gas stations and grocery stores). Some of these non-agricultural firms also utilize amenity inputs from the Salton Sea (for example, scenic quality) in their production 
processes. All three groups (consumers, agricultural and non-agricultural firms) generate waste that is discharged to Salton Sea water and air basins. In addition to providing firms with factors of production, the lake also provides local and non-local residents with direct benefits in the forms of amenities, air quality, recreation (that is, activities not involving a recreation services firm), and ecosystem services.

Socioeconomic monitoring involves measuring variables in terms of specific units (for example, number of beach visitors, acre-feet of drainage, tons of lettuce, and so on) and, where appropriate and feasible, in terms of economic value (for example, gross regional product, willingness to pay for bird habitat preservation, consumer surplus from recreation activities, and so on). Undoubtedly, some of the relevant variables will overlap with other monitoring groups (for example, drainage, habitat, and fish and bird populations). Spatial distributions and temporal trends also will be incorporated to the extent that this information helps to refine and characterize the socioeconomic effects of changes in the lake. Some of the variables that could be monitored include the following:

- Demand for market goods and services. Investment in non-firm capital (for example, residential real estate, or public infrastructure).

- Demographics (for example, population, age, income, education, unemployment, crime, and so on).

- Demand for products derived from the Salton Sea.

- Agricultural firms.

- Employment: types and number of jobs, wage rates.

- Capital investment (for example, land in cultivation, or irrigation systems).

- Water imports, drainage, transfers.

- Demand for other factors of production.

- Crop production.

- Non-agricultural firms.

- Capital investment (for example, buildings).

- Waste generation.

- Production of goods and services.

- Production of recreation services.

- Hotels, resorts, spas, destination restaurants.

○ Golfing.

○ Beach activities including swimming.

○ Fishing, including charters.

○ Bird watching.

○ Hunting.

- Boating and water skiing, including boat rentals.

- Salton Sea water and air basins: non-economic variables with important economic implications.

- Amenities (and disamenities).

- Scenic quality. 
- Odor.

- Serenity.

- Air quality (health-related effects).

- Ecosystem services.

- Local climate regulation.

- Habitat for fish, bird populations.

\section{Socioeconomic Monitoring Activities}

The objective for monitoring socioeconomic conditions and changes within the region is to gain insight into the effects the restoration process and resulting changes to the Salton Sea have on communities that are within its catchment. There is little difference in purpose between socioeconomic monitoring and environmental monitoring because both attempt to understand how restoration efforts move the system toward or away from a desired state. Restoration is not without purpose; there are management, legal, and human motivations for choosing to restore any system. However, systems need to be restored holistically to include socioeconomic communities that have come to rely on them over time. Monitoring provides a means to document and track changes to socioeconomic systems that rely on the health and function of the Salton Sea ecosystem as do the flora and fauna that are at the forefront of the restoration efforts.

Monitoring activities described in this section possibly do not have the same level of specificity as in other sections (for example, hydrology and biology) of this report. A thorough retrospective analysis of existing socioeconomic data and data-collection activities is needed prior to the detailed identification (and prioritization) of socioeconomic monitoring in support of Salton Sea Ecosystem Restoration. This retrospective analysis is required, in part, because there has not been the same level of effort in socioeconomic monitoring for ecosystem restoration at the Salton Sea as there has been for monitoring the biologic and hydrologic components of the ecosystem. Subject to results of the retrospective analyses, monitoring the demographic, economic, and human-perception conditions is anticipated and described in the following sections.

\section{Demographic Monitoring}

The socioeconomic monitoring program will identify individuals and communities who could be affected by changes to the Salton Sea environment due to restoration activities. Changes in the demographic make-up of the region could have both positive and negative effects on communities and individuals. It is essential to monitor these changes over time to gain insight into how restoration of the lake affects a range of demographic and associated factors.

\section{Purpose and Justification}

Restoration of the Salton Sea has effects beyond the physical and biological resources of the region. The Salton Sea also is tied to the livelihood, health, and function of the communities that surround it. Restoration can have a variety of implications, both positive and negative, for these communities. Developing and carrying out a well-designed monitoring program that tracks changes to the socioeconomic character of the region provides investigators with the data needed to make observations and find correlations between restoration and community welfare as well as information with which to answer questions and base decisions that could arise during and after the restoration period. 
Questions to be answered include the following:

- Who is affected socially and economically by the Salton Sea?

- What is the nature of different groups' socioeconomic relationship (for example, farmers, tourismbased industries, or geothermal) to the lake?

- What are the socioeconomic drivers influencing where (geographically) these groups live and their interactions with the lake?

- How have these individuals and their communities experienced the effect of changes in the Salton Sea over time, and how do these differ on the basis of group, social, and economic position and status? Responses to these questions are obtained by identifying affected groups and then monitoring various indicators within these socioeconomic catchments over time.

Monitoring socioeconomic activity of people and communities, including patterns of mobility into and out of the socioeconomic catchments surrounding the Salton Sea, provides an understanding of changing environmental and social effects. Monitoring also can identify other communities and socioeconomic clusters affected by the physical, biological, and cultural condition of the Salton Sea.

\section{Locations}

Monitoring activities will initially take place within the hydrologically defined watershed of the Salton Sea. As initial data collection progresses, this boundary could require adjustments to ensure essential socioeconomic clusters lying somewhat beyond this boundary are not overlooked. It is good to note that a substantial amount of socioeconomic data were identified through the PEIR process. These and other existing data will be integrated into the socioeconomic monitoring efforts to the extent feasible. Existing data provide important information regarding pre-restoration conditions without the need for extensive primary data collection. Existing information will need to be supplemented with data collected at the local level (that is, for communities located within the defined watershed). Socioeconomic catchments will be identified by using geographic mapping of socioeconomic clusters and networks that are strongly tied to the region on the basis of the spatial and content analysis of social and economic data.

By utilizing a sociospatial sampling approach (sampling based on geographically important factors), the analysis would focus on identifying the various Salton Sea socioeconomic catchments throughout the region. This approach begins with the communities located within the defined watershed and then adjusts outward to include other closely associated communities as determined through socioeconomic network analysis of these clusters. Defining social and economic boundaries on the basis of data related to income, age, race, commerce, and trade and industry growth and development could provide a more complete understanding of the effects of changes to the lake over time.

\section{Period and Frequency}

The initial focus is to develop an understanding of the socioeconomic landscape and interactions between and among clusters. Because these data would complement information obtained through other aspects of the overall monitoring and assessment plan, it is essential to coordinate the scale and frequency of data collection across resource areas. Specific elements that relate to the socioeconomic component are likely to include (but are not limited to) the following: 
- Avian resources.

- Fisheries resources.

- Surface water.

- Groundwater.

- Geology and geography (particularly land use and land cover).

- Air quality.

After data on existing conditions are obtained, and geographic and conceptual network mapping is complete, specific socioeconomic data will be collected on a 2 -year cycle for the first 20 years and on a 5 to 10 -year cycle thereafter, as determined necessary to track how continued restoration or recovery of the lake and the surrounding region approaches the desired end state (ecosystem and socioeconomic functions). Since important aspects of socioeconomic data are collected during the United States Census every 10 years, these collection cycles will be planned to coincide with those years in which the Census takes place (for example, 2010, 2020, and so on) For those data types that require more intensive collection or analysis, efforts could require nearly continual collection to ensure they are available at the temporal resolution specified. Additional special studies could become necessary during this process if the monitoring data indicate some unexpected result or other external factors necessitate more detailed data for a particular resource area.

\section{Protocol and Data Collection}

A variety of sociospatial mapping methods and protocols will be required to acquire and compile the data. These protocols likely will need to incorporate a variety of techniques, including the following:

- Written surveys.

- Key-informant interviews.

- Focus groups.

- Sociospatial Grounded Theory.

- Geographic Information System (GIS) mapping:

- Sociospatial mapping of clusters, communities, and networks.

- Social Network Analysis (mapping and visualizing the connections among people, groups, and organizations).

- Public Participation GIS mapping methods.

- Field validation/ground-truthing sessions.

- Secondary data mapping and analysis (for example, U.S. Census, Economic Census).

To the extent feasible, secondary data will be incorporated into the monitoring protocol to minimize duplication with available data sources. Examples include government agency data products, such as the U.S. Census of population and economy, county and agency data on land use and zoning, and a variety of state and federal agencies with responsibility for collection or monitoring of biological and physical data related to these functions (for example, airborne contaminants that affect health of communities in the basin, biological resources that support tourism in the region, and so on).

Sociospatial grounded theory is an example of a multi-method approach employing a specific recommended methodology (Steinberg and Steinberg, 2006). Steps in this process include the following: 
1. Determine topic of interest.

2. Determine a geographic location of interest.

3. Collect the data and spatially link the data.

4. Geocode the data.

5. Ground truth the data (by using Public Participation GIS sessions).

6. Analyze the data and look for spatial and social patterns.

7. Generate theory (spatial and social).

By using this approach, relevant socioeconomic clusters and networks throughout the region can be identified and the relevant catchment area defined. Planning for data acquisition to coincide with the decadal census of the U.S. population is to begin as early as possible to develop a clear understanding of the specific sociospatial sampling and data collection protocols that will be necessary. An advance review of existing secondary data relating to socioeconomics (for example, population and economic census data, land-use maps, and other county records) will be completed for the Salton Sea region over the last 30 to 50 years at 10 year intervals. Depending on the degree of variability in these historic data, it will become clear how the stability of social and economic clusters and networks changed with the decline of the ecosystem and, thus, inform the specific design appropriate to monitor changes to these clusters and interactions as restoration progresses.

Data collection can be coordinated with the U.S. Census to identify current sociospatial clusters and networks and migratory patterns of individuals engaged in economic and social activities tied to the lake (for example, commuting within the basin to work in agricultural production or out of the basin to work in hotels in Palm Springs). Data collection will initially focus on those individuals living within the hydrologically defined basin, but this boundary could require minor adjustments based on preliminary data analysis.

The first necessary step will be to identify the various socioeconomic clusters either directly or indirectly related to the Salton Sea. Because these clusters exist in both geographic and conceptual space, the identified clusters can then be characterized by their demographic and economic qualities as well as their dependencies with the lake and the surrounding natural and cultural features, including physical, biological, and other essential characteristics (for example, casino workers, farmers, service industry workers). By examining where activities of people who live in the Salton Sea watershed take place (for example, where they live, work, recreate, or send their children to school) and identifying patterns of socioeconomic dependence with the lake, the necessary data will be provided for monitoring changes to these systems and the character of the socioeconomic clusters, relationships, and communities in and around the basin. Through this type of spatially based research, it will be feasible to monitor specific populations and how they affect and are affected (positively or negatively) by the changing conditions of the Salton Sea related to the restoration process over time.

Because socioeconomic changes are not generally uniform across populations, the effects of restoration efforts on communities in the region will be stratified and monitored in relation to demographic characteristics. A specific focus on ethnicity will be essential to account for different effects on particular groups over time. The Salton Sea has historic and ongoing relevance to Native American communities. Social class plays an important role in determining people's relationship to the Salton Sea, their mobility patterns, and participation in economic activities related to the Salton Sea. The poor often experience fewer economic options and, as a result, are more limited in their mobility patterns to work, recreate, and interact in the Salton Sea watershed and within Salton Sea socioeconomic catchments. 
Changing water levels due to restoration efforts could have significant effects on the use of cultural resources by Native American peoples who first inhabited this region. As the lake recedes or regions previously under water are exposed because of dikes or pumping of water, sites of cultural importance to Native Americans, such as former settlement sites, could be exposed. This has potential to raise issues of accessibility to cultural artifacts of importance to people of the region. Access can expose cultural sites in either a positive or negative manner; for example, it makes them available to Native Americans communities and benefits their traditional, cultural uses, but it also exposes sensitive sites to vandalism or artifact hunters. Such effects of restoration will be monitored throughout the process to understand if and how these changes affect Native Americans from a cultural and socioeconomic perspective.

An initial phase in preparation for data collection is to perform a review and do sociospatial mapping of secondary census and economic data to identify relevant patterns and flows of people and resources in the region. Mapping these data over the last 30 to 50 years can facilitate identification of patterns, including socioeconomic clusters, in and out-migration of various social and economic demographics, and relationships between these both within and external to the Salton Sea watershed. Conducting a spatial examination of demographic data could also help to assess current and potential future demand for resources, such as water, electricity, and air quality, by individuals and communities in the region.

Text-based news stories provide a rich data source. Historical news items can support and enhance information contained in datasets such as the U.S. population or economic census. Content analysis of news stories, selected to coincide with the dates of other data collection efforts, can facilitate a researcher's ability understand the groups affected, where they reside, what sorts of industries existed in the watershed, and how they have been affected by the changing condition of the Salton Sea over time. Looking forward, news stories will be compiled and analyzed to provide this same context to determine how changes due to restoration of the lake are perceived to affect the region.

Once data collection for a particular year is complete, primary themes emerging from the data will be mapped for sociospatial analysis in conjunction with the other data sources. This process involves tying assessment of news stories about the Salton Sea to a particular geographic place, both near the lake and farther away. Through such a process, a spatial portrayal of socioeconomic themes and topics relative to the lake can be examined in context of historic change as well as in relation to future changes in the region.

To obtain information on the relationships within and between the various socioeconomic clusters identified, focus group meetings will be scheduled with each cluster identified. The purpose of these meetings is to ground-truth secondary data that have been analyzed to verify that they accurately portray the actual socioeconomic situation and economic clusters perceived by those individuals and communities within the Salton Sea region.

Interviews could portray the socioeconomic issues by place, both proximate to the lake and nonproximate to the lake, and would identify key socioeconomic issues for people living in the Salton Sea watershed. Interviews can also provide information to help determine/define the Salton Sea socioeconomic catchments. Key informant interviews will take place with individuals viewed as local "experts" on a topic or issue and who represent the range of socioeconomic groups within the defined region. The goal of these interviews is to determine how people are tied in an economic and social capacity to the lake, and how they perceive the changes taking place related to where they live, work (how far do they travel to work), and recreate in terms of the lake between the times of data collection efforts. In the first year, such information will be used to identify boundaries of influence of the Salton Sea and to define the resulting socioeconomic catchment. Key informants could include elected 
officials, community leaders, key community members, or other individuals with particular insight about their community or particular group.

As with content analysis for news stories, data from key informant interviews will be mapped to highlight locations of socioeconomic catchments and their characteristics.

On the basis of insights from analysis of key informant interviews, a survey of a stratified sample of members representing each of the socioeconomic clusters can be developed and administered to further examine and assess themes and patterns identified in those interviews. This involves collecting data from the clusters of people who live both proximate to the lake and those who live farther away from the lake but who are a part of the Salton Sea socioeconomic catchment. This provides a basis for determining how people's socioeconomic lives are tied to the quality and health of the Salton Sea ecosystem and watershed.

As with the other forms of survey data, key themes can be sociospatially mapped to identify and highlight emerging socioeconomic clusters and influences affecting individuals and communities within the Salton Sea socioeconomic catchment. These could include clusters both near the lake and elsewhere. Social or economic proximity can be a long distance geographically, but socioeconomically there can be related systems closely tied to or associated with the quality and health of the Salton Sea ecosystem.

The ultimate objective of this data collection is to examine relationships among various socioeconomic clusters and communities and how they are affected, either positively or negatively, by changing conditions of the Salton Sea. A spatial analysis of the networks, mobility patterns, and economic dependence can help track where and how people are engaging in socioeconomic activities regionally and how changes in the system affect the overall health and function of these communities. Socioeconomic monitoring can help to target clusters that are affected by restoration disproportionately or fail to improve in condition at rates observed for other clusters. This type of monitoring and analysis can help community members, community and regional planners, economic development agencies, and other decision makers target their efforts to maximize the benefits of restoration of the Salton Sea's physical and biological characteristics in relation to supporting the socioeconomic situation for people who live within its socioeconomic catchment.

\section{Data Analysis}

Data analysis will consist of two related types: statistical and sociospatial. Statistical analysis will be used to document descriptive information initially (pre-restoration) and on an ongoing basis, as new data are obtained and collected throughout the restoration process. As multiple years of data become available, additional analysis of changes and trends in the data will be explored. Where appropriate, additional analysis of socioeconomic data in conjunction with data from other aspects of the program will be explored (for example, correlations between changes to the biological resources as they relate to economic conditions). The second approach to data analysis would be sociospatial. Whereas standard statistical analysis has potential to show relationships and trends, there is an inherent spatial component to much of the data that would be obtained during the restoration period. By using GIS in conjunction with geospatial analysis techniques, the relationships of social and economic data in space and time could reveal additional patterns and changes resulting from the restoration process. By using GIS, additional variables can be derived for this purpose by exploring spatial relationships, distances and directions of parameters and their effects on the socioeconomic character of the region.

All of the monitoring data collected will be spatially coded and analyzed, including data obtained for the socioeconomic monitoring element. In some cases, this will be data collected under another part of the monitoring process; for example, data relating to ecological conditions will be available to examine in conjunction with socioeconomic data relating to socioeconomic health of communities surrounding the lake. The socioeconomic data will pair especially well with the 
environmental data on air and water quality, as fluctuations in those factors have both direct and indirect ties with socioeconomic issues. Examples of socioeconomic changes observed could include expansion of tourism and recreation industries that rely on vibrant communities of resident and migratory birds; breathable, fresh air; and increased desirability of the region for housing and jobs based on existing economic clusters such as agriculture or new clusters built on the availability of geothermal power and other support services.

\section{Quality Assurance}

Typically, socioeconomic data are considered sensitive information and, therefore, are subject to a reasonable degree of confidentiality. For this reason, such data usually are aggregated to ensure an appropriate degree of protection of individuals and communities included in the monitoring and assessment plan. Because the population density in the region surrounding the Salton Sea is relatively low, in many areas census block/block groups could prove to be insufficient to maintain the required data resolution. Where existing units of analysis, such as provided by the census, are appropriate, they will be considered an appropriate geographic unit of analysis, although other options could need to be considered to ensure utility of the data for long-term monitoring. Data that are associated with defined geographic units will be collected at a resolution appropriate to represent the unit.

Data collected by using survey methods requires a sufficient sample size to avoid bias in the data and to ensure the required statistical power for analysis. When surveys are an element of data collection, they will be peer-reviewed and pilot tested to ensure questions are appropriate for measuring the intended parameter. Field staff performing interviews with key informants or focus groups, or who are engaging in participatory mapping will be appropriately trained to ensure consistency both within and between data collection activities.

A mixed-method approach, reliant on both quantitative and qualitative data and collected from a variety of sources, is a robust means for collection of data essential to monitoring of the socioeconomic status of communities that are tied to the Salton Sea. One essential element of quality assurance and quality control (QA/QC) is to have a means of validating these data. One QA/QC technique is to hold a series of public meetings with a sample of the identified socioeconomic clusters to validate data and findings and allow for public input to further refine the data and findings. To maintain the spatial integrity of these data, a public participation GIS (PPGIS) technique is useful. Public participation GIS is a process where individuals interact with the data and associated information in the form of maps, which can be annotated by the participants to clarify, add, or delete information relating to the topics and data of interest. By adding their personal-on-the-ground experiences, the data reflect on-the-ground realities as perceived by the communities of interest, which could otherwise be overlooked by traditional data collection techniques carried out at a regional level and necessarily lower granularity. Participants in PPGIS activities are asked to mark data directly on the maps, or with the support of interactive mapping technologies, for capture into the geospatial database.

As mentioned in the earlier discussion on data collection and analysis, it is important to groundtruth data with the communities in the region to ensure that the compiled data align with on-the-ground realities as perceived and experienced by the communities themselves.

\section{Data Reporting}

Annual reports of all data collected as part of socioeconomic surveys will be published online, in paper form, or both. Any environmental attributes collected during the surveys also will be included in the data reports. When possible, data will be published in integrated annual data reports containing data collected by all disciplines. Some data (such as Census information) will be collected by others as part of ongoing activities with potentially differing goals and objectives. Data will be displayed in GIS 
formats when appropriate. These reports and other activities will be referenced in the annual data reports anticipated as part of this MAP. All data will be entered into the database management system. Recommendations for improvement of survey protocols also will be included in the annual reports.

\section{Overview of Similar Current Monitoring Activities}

No similar data collection programs are underway in the region. However, there are a variety of existing data sources that will provide important information for monitoring of the socioeconomic conditions and changes in the Salton Sea region. These include data collected at a variety of governmental levels, including those data maintained by federal, state, regional, and local government agencies. As is true of any existing data source, information was typically collected with a different purpose in mind and is unlikely to capture all of the required information identified previously. However, it is important that a retrospective analysis of existing sources be performed as soon as feasible to determine what is known, which of these sources can provide portions of the data necessary for monitoring, and where these sources will need to be supplemented with additional data.

Additionally, performing a retrospective analysis at the outset will provide important information and insight into the likely socioeconomic clusters to be monitored as well as clusters that could have existed in the past, but subsequently disappeared as the conditions of the Salton Sea and its surrounding watershed changed. With restoration efforts attempting to move the condition of the lake in a different direction, it is possible some of these former socioeconomic clusters could reemerge. Therefore, the historical context provides a basis for developing the appropriate metrics and sample design and potentially can demonstrate where and how these data from other social, economic, and other data categories can inform the monitoring process and eliminate data redundancy.

\section{Economic Monitoring}

\section{Purpose and Justification}

Future changes in the Salton Sea will produce significant changes in economic characteristics of the human population. Such changes can be perceived as beneficial or detrimental, depending on the perspective and interests of the affected population. Monitoring economic change is essential to understanding public perception and to develop effective communication strategies.

The primary need is to establish historic and existing conditions data for key economic indicators. This will facilitate an examination of changes related to or resulting from future changes in the Salton Sea. Key characteristics include, but are not limited to, perceived value of restoration, supply and value of ecosystem services, opportunities for and value of recreational activities, condition of the agricultural economy and the distribution of agricultural income, availability and value of geothermal energy, air quality related health and amenity effects, property values, and spill-over effects in the local secondary economy.

Collection of retrospective and existing-conditions data and establishment of an ongoing monitoring plan will enable tracking of the major economic effects of changes in the lake both spatially and temporally. Anticipated effects of changes in the lake and actual effects can be compared and used to guide specific decisions ensuring effective use of public funds, and to communicate to the public.

\section{Locations}

Monitoring data will be geo-referenced and focused on the Coachella and Imperial Valleys. Additionally, economic monitoring can track the greater sphere of influence of changes in the lake. Perceptions and opinions of residents throughout California and even the U.S. could be assessed to identify changes in the economic sphere of influence of the Salton Sea. 


\section{Period and Frequency}

Monitoring will take place at least annually, and perhaps semi-annually or even quarterly, particularly for economic sectors with natural business cycles lasting 1 year or less (for example, agriculture, tourism). Care will be taken to avoid observing a sector only at its zenith or nadir.

\section{Protocol and Data Collection}

Protocols for data collection during economic monitoring activities will be identified and documented in either a supplemental or additional report.

\section{Data Analysis}

Analysis methods for data collected during economic monitoring activities will be identified and documented in either a supplemental or additional report.

\section{Quality Assurance}

Methods to ensure data quality will be identified and documented in either a supplemental or additional report.

\section{Data Reporting}

Reporting requirements for economic monitoring activities will be identified and documented in either a supplemental or additional report.

\section{Overview of Similar Current Monitoring Activities}

Limited data are available at this time. Some economic statistics for the local economy exist and are compiled regularly by appropriate government agencies. Linkages with the larger regional, State, and national economies remain largely uncertain or unknown, however.

\section{Human-Perception Monitoring}

\section{Purpose and Justification}

Because the Salton Sea restoration project is as much a social endeavor as it is an engineering and ecological one, it is important to monitor public perception of the restoration. Doing so provides stakeholders with two types of critical, ongoing feedback. First, it measures the progress of public outreach efforts. This includes how well the restoration project is communicated to the public, and how well the public understands their potential participation in the process. Second, it measures public values and preferences for the project. Such information can help project leaders implement project elements that better satisfy stakeholder needs. In short, monitoring public perceptions helps ensure the engagement of stakeholders in the project, which increases the likelihood of overall success.

\section{Locations}

Public-perception monitoring will be performed in two geographic areas: the communities surrounding the Salton Sea, including the residents of Imperial and Coachella Valleys, and the broader State of California.

\section{Period and Frequency}

As with any monitoring program, it is important to establish existing perceptions. By comparing data over time against the historic and existing conditions, it is possible to identify trends. The existing conditions study will begin with a series of focus groups with individuals chosen from target populations. Focus groups will certainly be performed with people who are residents of the Imperial and 
Coachella Valleys, but such initial studies are also important for populations around the State. The focus groups serve as means to identify essential components of perception, as well as test for key terms that can be used in the quantitative studies. Next, the existing-conditions study will also include a comprehensive survey of samples from areas throughout California, with oversampling in the Imperial and Coachella Valleys. The existing-conditions study will be completed before the development of federal and State environmental documents (that is, Environmental Impact Statement and Environmental Impact Report). Doing so not only provides a better pre-project measure of perceptions, but it can also help the development of the environmental documents by identifying potential criteria of evaluation, project options, and outreach strategies.

Monitoring will be performed following three tracks (see table 18). 1) Focus groups - the goal would be to perform focus groups before the comprehensive survey and to repeat focus group analysis intermittently throughout the period of monitoring. 2) Survey - the goal is to perform an initial survey, followed by comparison surveys every 2 years. However, if major changes are expected either in the restoration project or public perception, it is possible to perform a general or target survey on a shorter time-step to obtain quantitative data. 3) Qualitative interview data collection would follow the comprehensive existing-conditions survey. It is expected that qualitative data will be collected throughout the period of monitoring, but that an intensive interview schedule will be maintained on at least an alternating year routine. The idea of the qualitative data collection is to use interviews to write individual case studies to illustrate findings from the survey.

\section{Protocol and Data Collection}

Monitoring will be quantitative, qualitative, and spatial. Quantitative monitoring w includes measurement of citizen perceptions through surveys. Qualitative monitoring consists of focus groups and public participation forums, including public participation GIS and interviews with those populations closest to the Salton Sea. The quantitative data allows for trend-tracking and quick reference. The qualitative data allows managers to understand the circumstances underlying values, preferences, and preference formation. The data could provide a more in-depth understanding of the lake/public relationship, especially for groups living within close proximity to the lake. Public participation GIS allows the proximate public to provide targeted feedback concerning their spatially based-perceptions of restoration efforts and helps to identify and monitor public perception in an ongoing basis. Targeted face-to-face and/or phone interviews provide the proximate population, especially the Spanish speaking and less educated, the opportunity to share their perceptions. Moreover, qualitative data provide real-life examples that could be used to craft outreach programs related to the Salton Sea restoration effort specific to different populations. Finally, both quantitative and qualitative data can be assessed spatially to create a more holistic understanding of the people, place, and their environment.

Furthermore, the monitoring effort will be both longitudinal and cross-sectional. Regular, periodic measurements of public perceptions allow officials to gauge trends, especially as they change with the progress of the restoration. Measurements across different groups at the same times allow officials to compare perceptions among different stakeholders. Such groups are not only geographic (for example, different metropolitan areas) but also demographic (for example, different social and economic classifications).

Approval of the perception studies from the Office of Management and Budget (under the auspices of the Paperwork Reduction Act) is required because the studies would be performed, at least in part, with federal funds and the results of the analyses could be used by federal agencies in their decision-making. The U. S. Geological Survey has extensive experience in obtaining OMB clearance for studies of this kind. The objective is to seek a generic clearance so that individual studies could be 
initiated as needed. It is anticipated that a generic clearance would require from 8 to 12 months after initiation of the process.

Quantitative survey data collection will be guided by protocols set forward in the work of Dillman (2000). Spatial analysis of survey questions will draw from various texts on spatial statistics, such as Mitchell (2005). Qualitative data collection will be guided by protocols established in Berg (2001) and Yin (1994). The spatial analysis of qualitative data will draw from Sociospatial Grounded Theory, as outlined in Steinberg and Steinberg (2006).

Perceptions most relevant to the planning and implementation of this project include periodic measurement of the public's knowledge, values, preferences, and uses as they relate to the future of the project area. It is important to distinguish differences based on geographic location between a "proximate public" (those living in close proximity to the lake) compared to a non-proximate public. People living in closer proximity to the lake, where it is part of their daily environment, have different perceptions and values of it than those living elsewhere.

First, the restoration effort needs to gauge citizens' knowledge of the Salton Sea and its restoration. It is vitally important to understand what people believe is happening with the restoration. Do they understand its purpose? Can they identify progress? Who are the stakeholders? Where are stakeholders located? Closely related to these questions are measurements of how they have come to these levels of understanding. Where do they get their information? What sources do they find trustworthy? Officials who are guiding the restoration effort need this information so they can better plan targeted outreach efforts, explain the project, and respond effectively to changes in plans or in the way citizens perceive the restoration project.

Second, the restoration effort needs to know the things that people value most about the Salton Sea and how those values change over time. What esthetic values do different stakeholder groups hold for the lake? How strong is the sense of place, and sense of community, among seaside villages? Have these increased or decreased with the restoration? On a larger scale, how are the restoration efforts affecting non-proximate stakeholder groups who also value the Salton Sea? In a related dimension, the restoration effort needs to know people's preferences toward the lake and the restoration project. Knowing what people value about the lake will enable officials to adjust plans to satisfy those values and explain parts of the plan that do not directly connect to the values but support them indirectly. Monitoring values will quantify one of the big drivers for change in the Salton Sea system. The restoration project depends on citizen support not only for the initial restoration itself, but also for the on-going maintenance of the lake. Citizen values change, and managers will need to adapt to those changes through the years. Furthermore, there are different populations and groups who value the lake, some of whom are proximate and others who live in another part of the State. The value of the Salton Sea extends beyond the residents who live immediately close to the lake.

Third, the restoration effort needs to know the public's preferences for the project in order to develop a plan that meets the desires of stakeholders. Which of the multiple project goals are most important to the public? Which project options are most preferred? Who prefers which options? Answers to these questions will help officials develop project elements that reflect the public's preferences. It also allows them to prioritize competing elements when project resources are scarce.

Fourth, use is another big driver of change. Monitoring how the lake is used by locals and visitors as the restoration effort progresses is very important. Understanding use trends contributes to application of adaptive management and to development of economic estimates.

Spatial analysis of these data consists of questions about public perception, which could be carried out at a focus group session. Proximate Salton Sea residents would be provided with maps of the area including the restoration efforts. Public participation GIS allows residents to provide targeted and 
ongoing feedback on their perceptions (for example, what they have concerns about, what they value, perceptions of how community is affected) relative to Salton Sea restoration efforts. Spatial analysis will also consist of asking and analyzing geographically based questions on the survey that can be used to better understand how perceptions of the lake vary based on geographic locations of both the proximate and non-proximate public relative to the Salton Sea.

Data Analysis

Analysis methods for data collected during human-perception monitoring activities will be identified in the retrospective analyses and documented in either a supplemental or additional report.

Quality Assurance

The monitoring of public perceptions will meet academic standards in the social sciences, and will be vetted through peer review. The scope of the review will include data collection, analytic methods, and findings. Furthermore, datasets could be made available to the public, as long as the privacy of human subjects is fully protected. Peer review and approval will follow the U.S. Geological Survey Fundamental Science Practices policy.

Data Reporting

Reporting requirements for human-perception monitoring activities will be identified in the retrospective analyses and documented in either a supplemental or additional report.

Overview of Similar Current Monitoring Activities

This monitoring of perceptions represents a completely original collection of primary data through focus groups and surveys. We are unaware of any current monitoring of human perceptions regarding the Salton Sea. 


\section{Data Integration, Assessment, and Prioritization}

\section{Integration}

Integration of results and data from monitoring activities is a critical part of this MAP. Integration will be one of the responsibilities of all personnel working on restoration of the ecosystem. Through integration, scientists and decision-makers will be better able to effectively and efficiently coordinate field activities for maximum savings of time and money. In addition, the integration of protocols, standards, and practices will help assure that data will be scientifically valid and usable for the widest possible variety of assessments and uses. Integration starts in the planning phases and extends throughout the collection, interpretation, reporting, assessment, and adaptive management parts of the restoration process.

\section{Data Assessment}

Assessment focuses on the query and retrieval of data stored or accessible from a centralized data retrieval system and applying statistical and other analytical techniques to the data. This step helps to simplify the collected data, test for change and differences, develop and test hypotheses, and evaluate uncertainty. It is through rigorous assessment that progress toward restoration goals can be measured, and it is through regularly scheduled assessments that adaptive management can be effectively applied. As Williams and others (2007, p. 23) note, "The success of an adaptive management project requires an adequate understanding of the resource issues and the dedication to stay abreast of new information." Assessment is critical to ensure that data are used and not just dumped into a database and forgotten. Assessment of data collected as part of this plan will be used to provide answers to questions posed by managers, policy-makers, and scientists.

\section{Data Prioritization}

The information presented in this plan is intended to be used in the development of a detailed implementation plan. A key part of the implementation plan will be the objective consideration of priorities for data collection. These priorities include immediacy (human health and safety, and so on), scientific need, management need, cost, ease (the low-hanging fruit principle), and availability of an alternative (if any). The prioritization process will also need to be responsive to political influences and needs. An advantage, however, of the rigorous technical integration and assessment process is the ability to provide incentive and justification for the prioritization of activities and the efficient application and focus of political support.

Priorities for integration will be guided by the overall objectives and needs identified for ecosystem restoration in general. Priorities for short-term monitoring efforts also will be strongly influenced by needs to document and assess status and trends. The highest priority questions identified by each focus technical group will be used to establish priorities within the existing available funding.

A retrospective analysis of existing information is needed prior to any potential field work to objectively and fully identify data gaps. In general, a synoptic (snapshot) survey of existing hydrologic, biologic, geographic, geologic, and air-quality conditions is a high priority and needs to be addressed as soon as possible. Additional monitoring, as outlined in this document, will allow for an assessment of the variability of each factor and an analysis of trends or changes from existing conditions. 


\section{Data Management}

\section{Overview}

The Salton Sea Ecosystem Monitoring and Assessment Plan (MAP) includes a data collection, analysis, and management system that will help inform and guide potential management actions for the restoration of the Salton Sea ecosystem. The data-management element of the MAP is the responsibility of the California Department of Fish and Game, in coordination with the MAP Science Coordinator, to receive, archive, and disseminate many of the datasets generated through the monitoring program. Because many of the environmental and socioeconomic assessment and monitoring activities contained in the Salton Sea MAP involve the collection of geospatial data, it is anticipated that GIS will be a primary platform used to manage, organize, analyze, and express these data. The long-term utility of geospatial data requires significant attention to the development of high-quality metadata, data lineage tracking, and quality assurance/quality control (QA/QC) of datasets.

Data collected by scientists working in the Salton Sea area will conform to the guidelines and standards discussed previously in the discipline-specific sections of this MAP. The data will be available through their own agency and or through the CDFW BIOS. Scientists will be encouraged to use the BIOS, but in some instances, it may be more practical to use agency databases. Ultimately, a distributed database is envisioned where the physical location of the data are transparent to the user.

Effective data management will be integral to the success of the MAP and, ultimately, to the success of the decision making and restoration activities that the monitoring data will inform. Data management complexity increases for long-term and broad-scale comparative studies and assessments in which scientists from numerous disciplines can be involved for long periods to address a complex series of questions or hypotheses. This necessitates increased attention to adequate metadata, QA/QC, archiving, and methods standardization (Michener, 1997).

\section{Objectives}

The principal objectives of the data management element are the following:

- Establishing and enforcing standards for data documentation.

- Establishing data transfer and storage protocol.

- Making data easily accessible and in a useable format.

\section{Data Management Requirements}

The following data management activities for the MAP will be the responsibility of each partner agency and can include data and database design and implementation, including data entry sheet design; data collection protocols; data entry; QA/QC; data processing; summarization of data into reports, charts, graphs, and so on; and data documentation (metadata).

Data collected under the various monitoring plans will be housed and made accessible either through the CDFW or the appropriate agency. Data collection will follow the protocols laid out under the individual monitoring plans. All deliverable products will contain metadata that meet the CDFW's metadata standards as defined in appendix 9. For any new data collection, the CDFW will review the 
database design to ascertain compatibility in BIOS and to ensure that metadata have been identified. For any existing data collection, review of the database and metadata will be made and suggestions given where appropriate. Data that will be housed within another agency will need to follow the requirements and protocol of that agency.

Agencies submitting data to the CDFW will be required to deliver the data in an acceptable digital format (GIS data will need to be in an Esri format, databases in either Access or Excel, word documents in a Microsoft format or in a searchable PDF format, and so on) and provide metadata that meets both the FGDC guidelines and the CDFW's own standards (see appendix 10 "Standards That Enable You to Contribute Your Data to Larger Data Sets").

Agencies must consider the needs of both the public and the technical user when compiling, assembling, and disseminating data. For example, the public generally prefers to see data summarized into reports, maps, visual aids, figures, and so on; whereas, technical users prefer to see the raw datasets, technical articles, manuals, protocols, and so on. Where applicable, data derived products, such as reports, summaries, graphs, and so on, will be provided to the CDFW.

\section{Agency Coordination}

A memorandum of understanding (MOU) will be drafted between the CDFW and each agency that will be contributing data to the MAP to address data exchange requirements. These memoranda serve as data sharing agreements to outline initial roles and responsibilities for development and use of the Salton Sea data and information management, stewardship, and dissemination program. An MOU template is included as appendix 11.

\section{Quality Assurance and Control}

All data submitted to the $\mathrm{CDFW}$ will be subject to QA/QC in the following manner:

- Do the data have a projection and datum defined?

- Does the data table have any truncated field names; are there any value outliers; are the data consistent within a field; should any fields be re-ordered; if there is a related table does each table have a consistent key field to join the tables together?

- Are there any spatial outliers or are there any glaring coordinate errors (for example, do harbor seals show up in Death Valley, does a point with the attribute of Imperial County appear in Inyo County on the map)?

- Do the metadata meet both the FGDC and the CDFW's metadata standards? Do the metadata include key words, list all of the attributes and their associated definitions, and was a spell check completed?

The CDFW will provide support in the transfer of data; data will be backed-up on and off site on the basis of the rate of changes made to the data; versioning will be documented within the quality section of the metadata, and datasets will contain date stamps; access control will be enforced by securing the dataset location, and access will only be provided to authorized personnel.

\section{Storage and Dissemination}

The CDFW will store geographic data and databases that are submitted to them. Agencies collecting specialized data with intricate databases (such as the Air Resources Board and the State 
Water Resources Control Board) will maintain the data within their own database collection system. These data will need to meet those agencies' standards and protocols. It is anticipated that in the future, integration of specialized data housed in other departments will enable display of those data along with all of the other Salton Sea data in a single map viewer through the use of distributed databases.

Geographic data that are collected for the Salton Sea MAP and provided to CDFW will be archived and made accessible through the CDFW's BIOS internet map viewer, and all non-geographic data will be made accessible through the CDFW's document library. In order for data sharing to be successful, the guidelines laid out in the" Data Management" section must be met, MOUs must be in order, and all contracts must contain the following verbiage:

All data collected and created for this contract are a required deliverable of this contract and will become the property of the State of California, and not of the contracted party. A condition of final payment on this contract shall include the delivery of all related data. Spatial data should be delivered in an ESRI useable format where applicable and documented with metadata in accordance with minimum BIOS metadata standards (http://bios.dfg.ca.gov/metadata.asp) and FGDC metadata standards (http://www.fgdc.gov/metadata/documents/workbook_0501_bmk.pdf).

All documentation and reports provided to the CDFW will be made accessible through the CDFW's document library. The document library provides a single storage area for a variety of documents and is available to the public through the internet. The document library provides an easy way to search through all of the stored documents to find a particular topic. Depending on the need, each individual document can be made public or can be secured so that only specific users can view it. Metadata for spatial and non-spatial data will be distributed to metadata clearinghouses to provide access to a wider audience. Examples of metadata clearinghouses include, but are not limited to, the National Biological Information Infrastructure (NBII), California Environmental Resources Evaluation System (CERES), and the National Spatial Data Infrastructure (NSDI). 


\section{References Cited}

Anderson, M.A., Whiteaker, L., Wakefield, E. and Amrhein, C., 2008, Properties and distribution of sediment in the Salton Sea, California: an assessment of predictive models: Hydrobiologia, v. 604, p. 97-110.

Anderson, M.A. and C. Amrhein, 2002, Nutrient Cycling in the Salton Sea, Final Report to Salton Sea Authority: University of California Riverside, Department of Environmental Science, 86 p.

Barker, C.E., 1995, Salton Trough Province (016), in Gautier, D. L., Dolton, G.L., Takahashi, K.I., and Varnes, K.L., eds., 1995 National assessment of United States oil and gas resources - results, methodology and supporting data: U.S. Geological Survey Digital Data Series DDS-30, Release 2, one CD-ROM.

Berg, B.L., 2001, Qualitative Research Methods for the Social Sciences (4d ed.): Needham Heights, Mass., Allyn and Bacon, 304 p.

Besser, J.M., Brumbaugh, W.G., Papoulias, D.M., Ivey, C.D., Kunz, J.L, Annis, M. and Ingersoll, C.G., 2012, Bioaccumulation and toxicity of selenium during a life-cycle exposure with desert pupfish (Cyprinodon macularius): U.S. Geological Survey Scientific Investigations Report 2012-5033, 30 p. with appendixes.

Byron, E.R., and Ohlendorf, H.M., 2007, Diffusive flux of selenium between lake sediments and overlying water: Assessing restoration alternatives for the Salton Sea: Lake and Reservoir Management, no. 23, p. 630-636.

California Department of Fish and Game, 2008, Salton Sea Fisheries Long-term Monitoring, Draft Quarterly Report, Fall 2008: Bermuda Dunes, Calif., Salton Sea Program, Department of Fish and Game.

California Department of Water Resources, 2003, California's Groundwater Bulletin 118, update 2003: Available at http://www.water.ca.gov/groundwater/bulletin118/update2003.cfm.

California Resources Agency, 2006, Salton Sea Ecosystem Restoration Program Draft Programmatic Environmental Impact Report, available at: http://www.water.ca.gov/saltonsea/peir/draft/

California Resources Agency, 2007, Salton Sea Ecosystem Restoration Program Final Programmatic Environmental Impact Report, vol. 1, available at http://www.water.ca.gov/saltonsea/documents/final_eir.cfm.

Chung, E., Schladow, S.G., Perez-Losada, Joaquim, and Robertson, D. M., 2008, A linked hydrodynamic and water quality model for the Salton Sea: Hydrobiologia, v. 604, p. 57-75.

Coachella Valley Water District, 2002a, Coachella Valley Final Water Management Plan.

Coachella Valley Water District, 2002b, Final Program Environmental Impact Report for Coachella Valley Water Management Plan and State Water Project Entitlement Transfer.

Conway, C.J., 2008, Standardized North American Marsh Bird Monitoring Protocols: Wildlife Research Report \#2008-01. U.S. Geological Survey, Arizona Cooperative Fish and Wildlife Research Unit, Tucson, Ariz.

Cook, C.B., 2000, Internal dynamics of a terminal basin lake: A numerical model for management of the Salton Sea: Davis, California, University of California, Davis, Ph.D. dissertation, 200 p.

Cook, C.B., Huston, D.W., Orlob, G.T., King, I.P. and Schladow, S.G, 1998, Salton Sea Project, Phase II Final Report: Data Collection and Analysis for Calibration and Verification of a Three-Dimensional Hydrodynamic Model, Report 98-2: Department of Civil and Environmental Engineering, University of California, Davis.

Cook, C.B., Orlob, G.T., and Huston, D.W., 2002, Simulation of wind-driven circulation in the Salton Sea: implications for indigenous ecosystems: Hydrobiologia, v. 473, p. 59-75. 
Detwiler, P.M., Coe, M.F., and Dexter, D.M., 2002, The benthic invertebrates of the Salton Sea: distribution and seasonal dynamics: Hydrobiologia, v. 473, p. 139-160.

Dillman, D.A, 2000, Mail and Internet Surveys - The Tailored Design Method (2 ${ }^{\text {nd }}$ ed.): New York, John Wiley \& Sons, Inc., 480 p.

Eccles, L.A., 1979, Pesticide residues in Agricultural drains, Southeastern Desert Area, California: U.S. Geological Survey Water Resources Investigations 79-16, 60 p.

Federal Register, 2009, Vol. 74, No. 231 /Thursday, December 3, 2009, Rules and Regulations, p. 63309.

Friend, Milton, 2002, Avian Disease at the Salton Sea: Hydrobiologia, v. 473, p. 293-306.

Hely, A.G., Hughes, G.H., and Irelan, Burdge, 1966, Hydrologic Regimen of the Salton Sea, California. U.S. Geological Survey Professional Paper 486-C, 32 p.

Highland, L.M., and Bobrowsky 2008, The landslide handbook--A guide to understanding landslides, Geological Survey Circular 1325, 129 p.

Holdren, G.C. and Montano, Andrew, 2002, Chemical and Physical Characteristics of the Salton Sea, California: Hydrobiologia, v. 473, p. 1-21.

Imperial Irrigation District and U.S. Bureau of Reclamation, 2002, Imperial Irrigation District Water Conservation and Transfer Project Draft Habitat Conservation Plan and Draft Environmental Impact Report/Environmental Impact Statement.

Keeney, Sharon, 2009, Desert Pupfish Surveys: Draft Memo to Files, Bermuda Dunes, Calif., Salton Sea Program, California Department of Fish and Game, Bermuda Dunes Office.

LFR Levine-Fricke, 1999, Synthesis Document of Current Information on the Sediment Physical Characteristics and Contaminants at the Salton Sea, Riverside and Imperial Counties, California, $16 \mathrm{p}$. Environmental Reconnaissance of the Salton Sea Sediment Contaminants. Prepared for the Salton Sea Authority

Loeltz, O. J., Ireland, B., Robison, J. H., and Olmsted, F. H., 1975, Geohydrologic reconnaissance of the Imperial Valley, California: U. S. Geological Survey Professional Paper 486-K, 54 p.

Martin, B.A., and Saiki, M.K., 2005, Relation of desert pupfish abundance to selected environmental variables in natural and manmade habitats in the Salton Sea basin: Environmental Biology of Fishes, v. 73, p. 97-107.

Martin, B.A., and Saiki, M.K., 2009, Trophic relationships of small nonnative fishes in a natural creek and several agricultural drains flowing into the Salton Sea and their potential effects on the endangered desert pupfish: The Southwestern Naturalist, v. 54, no. 2, p. 156-165.

May, T.W., Walther, M.J., and Brumbaugh, W.G., 2007, Selenium Concentrations in Irrigation Drain Inflows to the Salton Sea, California, October 2006 and January 2007: U.S. Geological Survey OpenFile Report 2007-1113, 18 p.

Mendelsohn, M.B., Boarman, W.I., and R.N. Fisher, 2006, Diversity patterns in the terrestrial avifauna of the Salton Sea, in Proceedings of the Third International Partners in Flight Conference, Asilomar, CA: Albany, Calif., USDA Forest Service Pacific Southwest Research Station Gen. Tech. Rep. PSWGTR-191, p. 617-620.

Michener, W.K., 1997, Quantitatively Evaluating Restoration Experiments: Research Design, Statistical Analysis, and Data Management Considerations: Restoration Ecology, v. 5 no. 4, p. 324-337.

Mitchell, Andy, 2005, The Esri Guide to GIS Analysis Volume 2: Spatial Measurements and Statistics: Redlands, California: Esri Press, 190 p.

Molina, K.C., 2004, Breeding larids of the Salton Sea: trends in population size and colony site occupation: Studies in Avian Biology, v. 27 p. 92-99. 
Molina, K.C., and Sturm, K.K., 2004, Annual colony site occupation and patterns of abundance of breeding cormorants, herons, and ibis at the Salton Sea: Studies in Avian Biology, v. 27, p. 42-51.

Montgomery Watson, 1996, County of Imperial, Imperial County Groundwater Study, prepared by Montgomery Watson Inc.

Office of Environmental Health Hazard Assessment, 2009, Safe Eating Guidelines for fish from the Salton Sea (Imperial and Riverside Counties) updated March 18, 2009: California Environmental Protection Agency, accessed October 18, 2009, available at http://oehha.ca.gov/fish/so_cal/saltonsea.html.

Patten, M.A., McCaskie, Guy, and Unitt, Philip, 2003, Birds of the Salton Sea: Status, biogeography, and ecology: Berkeley, Calif., University of California Press, 374 p.

Ralph, C.J., Geupel, G.R., Pyle, Peter, Martin, T.E., DeSante, D.F., 1993, Handbook of Field Methods for Monitoring Landbirds: Albany, Calif., USDA Forest Service Pacific Southwest Research Station General Technical Report PSW-GTR-144, 41 p.

Rantz, S.E. and others, 1982, Measurement and Computation of Streamflow, Volume 1, Measurement of Stage and Discharge and Volume 2, Computation of Discharge: U.S. Geological Survey Water Supply Paper 2175, 4 p.

Reese, B.K, 2007, Sulfur biogeochemistry of the Salton Sea, California, M.S. Thesis, University of California, Riverside, $92 \mathrm{p}$.

Riedel, Ralf, Caskey, Lucille, and Costa-Pierce, B.A., 2002, Fish biology and fisheries ecology of the Salton Sea, California: Hydrobiologia, v. 473, p. 229-244.

Roos, Maurice, 2005, Accounting for Climate Change, vol. 4 in California Water Plan Update 2005: California Department of Water Resources, Sacramento, California, p. 611-625. Available at http://www.waterplan.water.ca.gov/docs/cwpu2005/vol4/vol4-globalclimateaccountingforclimatechange.pdf

Rueda, F.J. and Schladow, Geoffrey, 2003, Dynamics of large polymictic lake II: numerical simulations: Journal of Hydraulic Engineering, v.129, no. 2, p. 91.

Saiki, M.K, 1990, Elemental concentrations in fishes from the Salton Sea, southeastern California: Water, Air, and Soil Pollution, v. 52, p. 41-56.

Saiki, M.K., 2007, Year 2 summary report; baseline selenium monitoring of agricultural drains operated by the Imperial Irrigation District in the Salton Sea basin: Imperial, Calif., Imperial Irrigation District, Water Department-Water Transfer, 70 p.

Saiki, M.K., Martin, B.A., and Anderson, T.W., 2011, Unusual Dominance by Desert Pupfish (Cyprinodon Macularius) in Experimental Ponds within the Salton Sea Basin: The Southwestern Naturalist, v. 56, no. 3, p. 385-392.

Saiki, M.K., Martin, B.A., and May T.W., 2010, Final report: Baseline selenium monitoring of agricultural drains operated by the Imperial Irrigation District in the Salton Sea Basin, California: U.S. Geological Survey Open-File Report 2010-1064, 102 p.

Saiki, M.K., Martin, B.A., and May, T.W., 2008, Year 3 summary report: baseline selenium monitoring of agricultural drains operated by the Imperial Irrigation District in the Salton Sea basin: Dixon, California: U.S. Geological Survey Open-File Report 2008-1271, 70 p.

Salton Sea Science Subcommittee, 2000, Strategic Science Plan, Salton Sea Restoration Project: La Quinta, Calif., Salton Sea Science Office, 122 p.

Schroeder, R.A. and Orem, W.H., 2000, Nutrient Dynamics in the Salton Basin - Implications from Calcium, Uranium, Molybdenum, and Selenium in Spring Meeting, American Geophysical Union, May 30-June 3, 2000: Washington, D.C. EOS Trans. Suppl. 81, no. 19. Abstract no. H31B-02, p. S196. 
Schroeder, R.A., Orem, W.H., and Kharaka, Y.K., 2002, Chemical Evolution of the Salton Sea, California: nutrient and selenium dynamics: Hydrobiologia, v. 473 p. 23-45.

Setmire, J.G., and Schroeder, R.A., 1998, Selenium and salinity concerns in the Salton Sea Area of California, in Environmental Chemistry of Selenium: New York, Marcel Dekker, Inc., p. 205-221.

Shuford, W.D., Warnock, Nils, Molina, K.C., Mulrooney, Brennan, and Black, A. E., 2000, Avifauna of the Salton Sea: Abundance, distribution, and annual phenology: Stinson Beach, Calif., Point Reyes Bird Observatory Contribution No. 931, 126 p.

Shuford, W.D., Warnock, Nils, Molina, K.C., and Sturm, K.K., 2002, The Salton Sea as critical habitat to migratory and resident waterbirds: Hydrobiologia, v. 473, p. 255-274.

Steinberg, S.J., and Steinberg, S.L, 2006, Geographic Information for the Social Sciences: Investigating Space and Place ( $1^{\text {st }}$ ed.): Thousand Oaks, Calif., Sage Publications, 272 p.

Tiffany, M.A., González, M.R., Swan, B.K., Reifel, K.M., Watts, J.M., and Hurlbert, S.H., 2007, Phytoplankton dynamics in the Salton Sea, California, 1997-1999: Lake and Reservoir Management, v. 23, p. 582-605.

Tiffany, M.A., Swan, B.K., Reifel, K.M., Watts, J.M., and Hurlbert, S.H., 2002, Metazooplankton dynamics in the Salton Sea, California, 1997-1999: Hydrobiologia, v. 473, p. 103-120.

Tokunaga, T.K., Pickering, I.J., and Brown, G.E. Jr. 1996. Selenium transformations in ponded sediments: Soil Science Society of America Journal, v. 60, p. 781-790.

Tompson, Andrew, Demir, Zafer, Moran, Jean, Mason, Denise, Wagoner, Jeff, Kollet, Stefan, Mansoor, Kayyum, and McKereghan, Peter, 2008, Groundwater Availability Within the Salton Sea Basin, Final Report (LLNL-TR-400426): Livermore, Calif., Lawrence Livermore National Laboratories, 156 p.

U.S. Bureau of Reclamation, 2000, Salton Sea Restoration Project Draft Environmental Impact Statement/Environmental Impact Report. Available at: http://www.usbr.gov/lc/region/saltnsea/deistoc.html

U.S. Environmental Protection Agency, 2006, Guidance on Systematic Planning Using the Data Quality Objectives Process EPA QA/G-4: Washington, D.C., Office of Environmental Information, EPA/240/B-06/001, $111 \mathrm{p}$.

Vogl, R.A., and Henry, R.N., 2002, Characteristics and contaminants of the Salton Sea Sediments: Hydrobiologia, v. 473, p. 47-54.

Wahl, K.L., Thomas, W.O. Jr., and Hirsch, R.M., 1995, Stream Gaging Program of the U.S. Geological Survey: U.S. Geological Survey Circular 1123.

Williams, B.K., Szaro, R.C., and Shapiro, C.D., 2007, Adaptive management: The U.S. Department of the Interior technical guide, Washington, D.C.: U.S. Department of the Interior.

Yin, R. K., 1994, Case Study Research Design and Methods, (2 ${ }^{\text {nd }}$ ed.), Thousand Oaks, Calif., Sage Publications, $192 \mathrm{p}$. 
Figures 


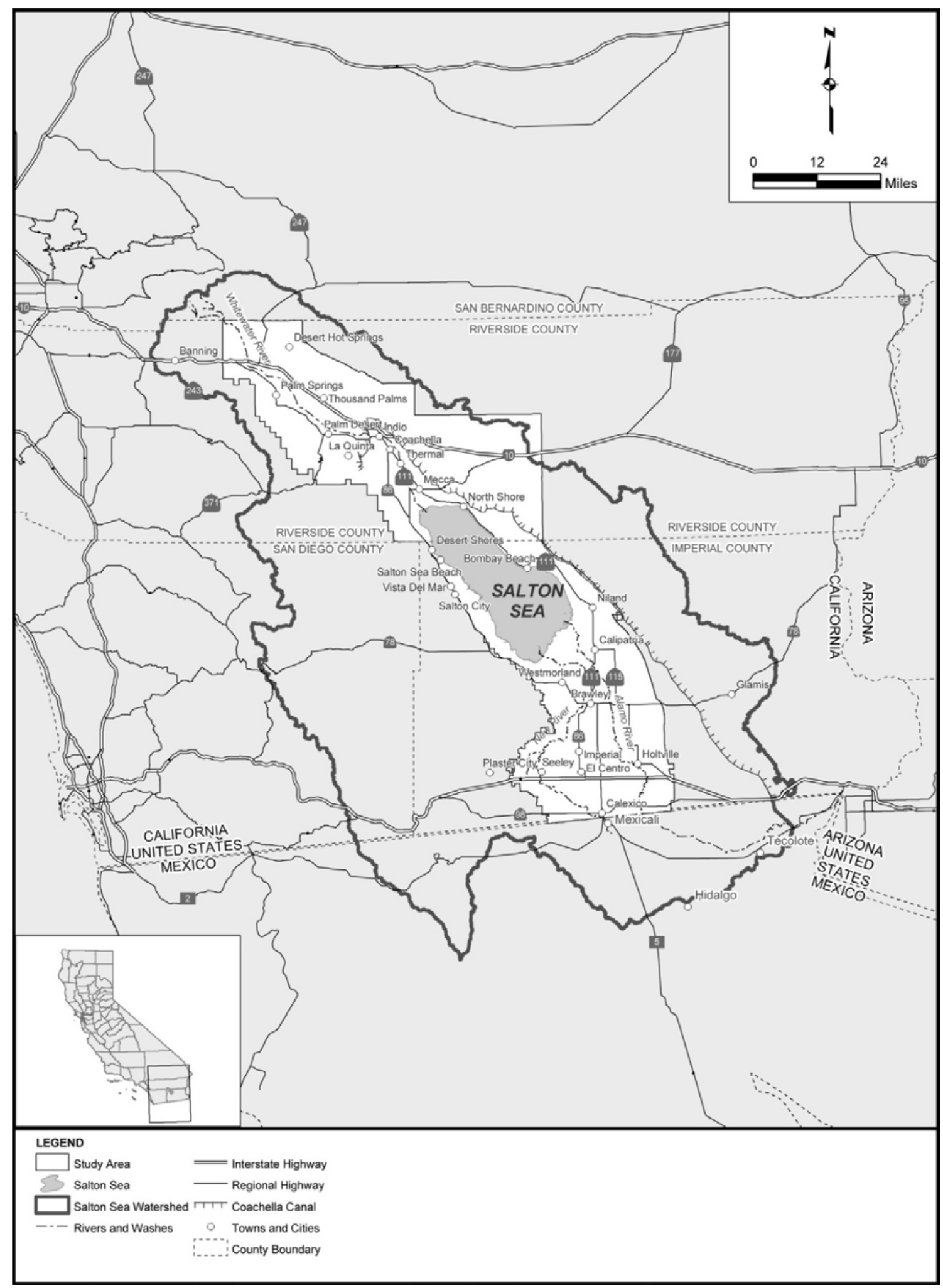

Figure 1. Study Area from Base California Resources Agency, 2006, Salton Sea Ecosystem Restoration Program Draft Programmatic Environmental Impact Report, figure 1-1, available online at URL http://www.water.ca.gov/saltonsea/peir/draft/ 


\section{Salton Sea Restoration Council Science Committee}

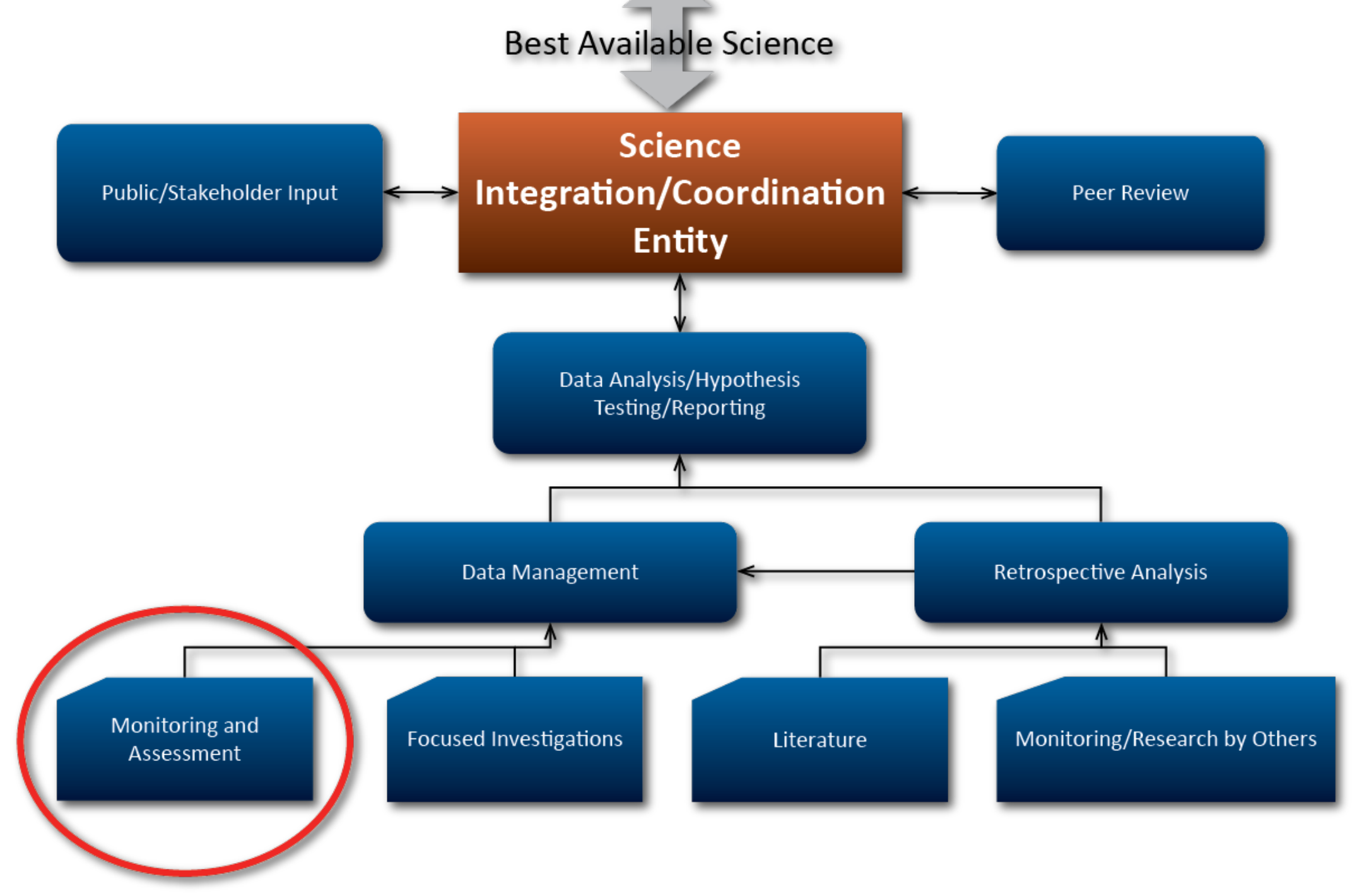

Figure 2. Conceptual framework for the science program 


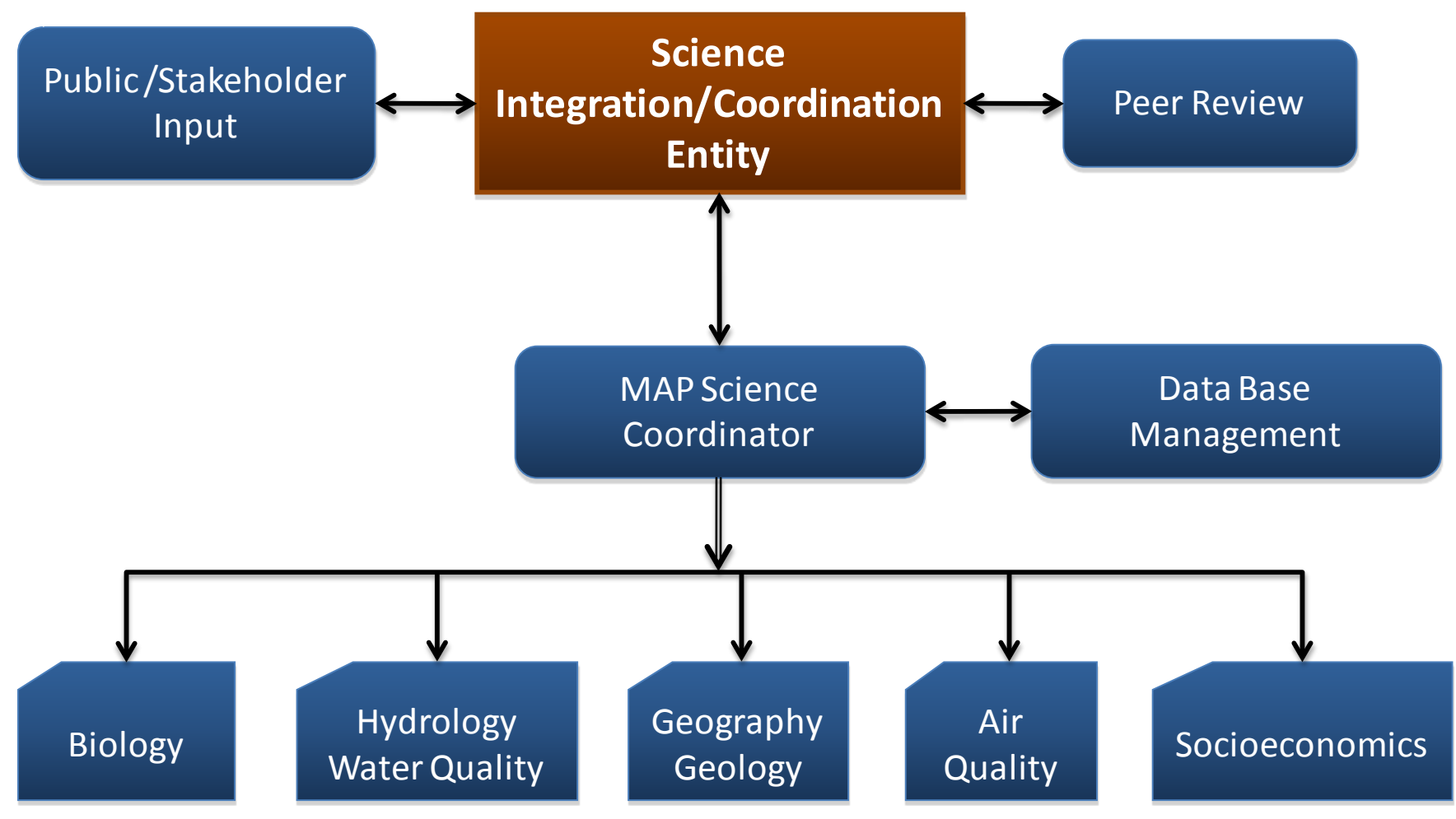

Figure 3. Potential organization of the Monitoring and Assessment Plan work group 


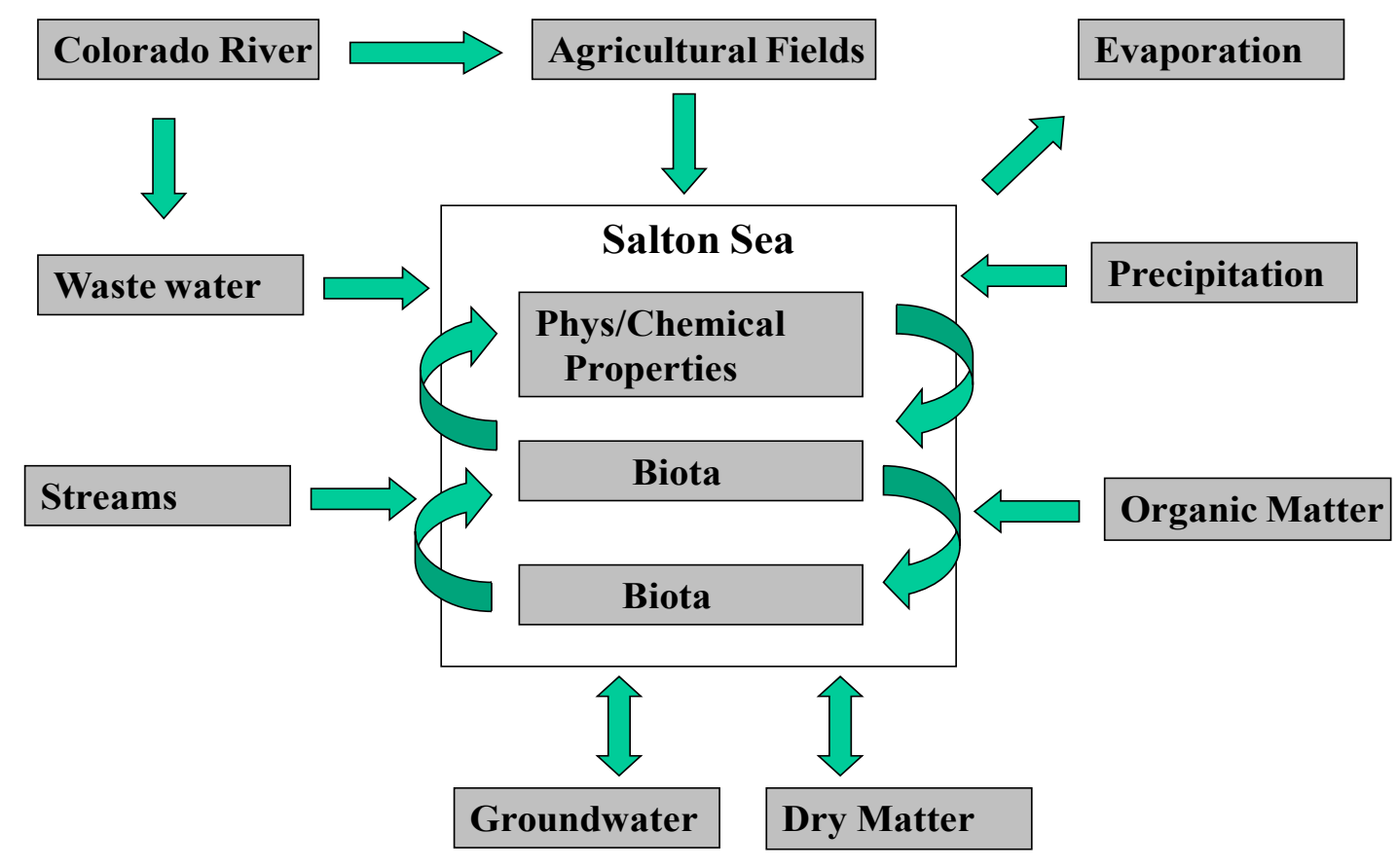

Figure 4. Conceptual Model for the Salton Sea Ecosystem. Modified from the Strategic Plan Salton Sea Restoration Project by the Salton Sea Science Subcommittee (2000), available online at URL http://www.usbr.gov//c/region/saltnsea/pdf_files/sciplan.pdf. 


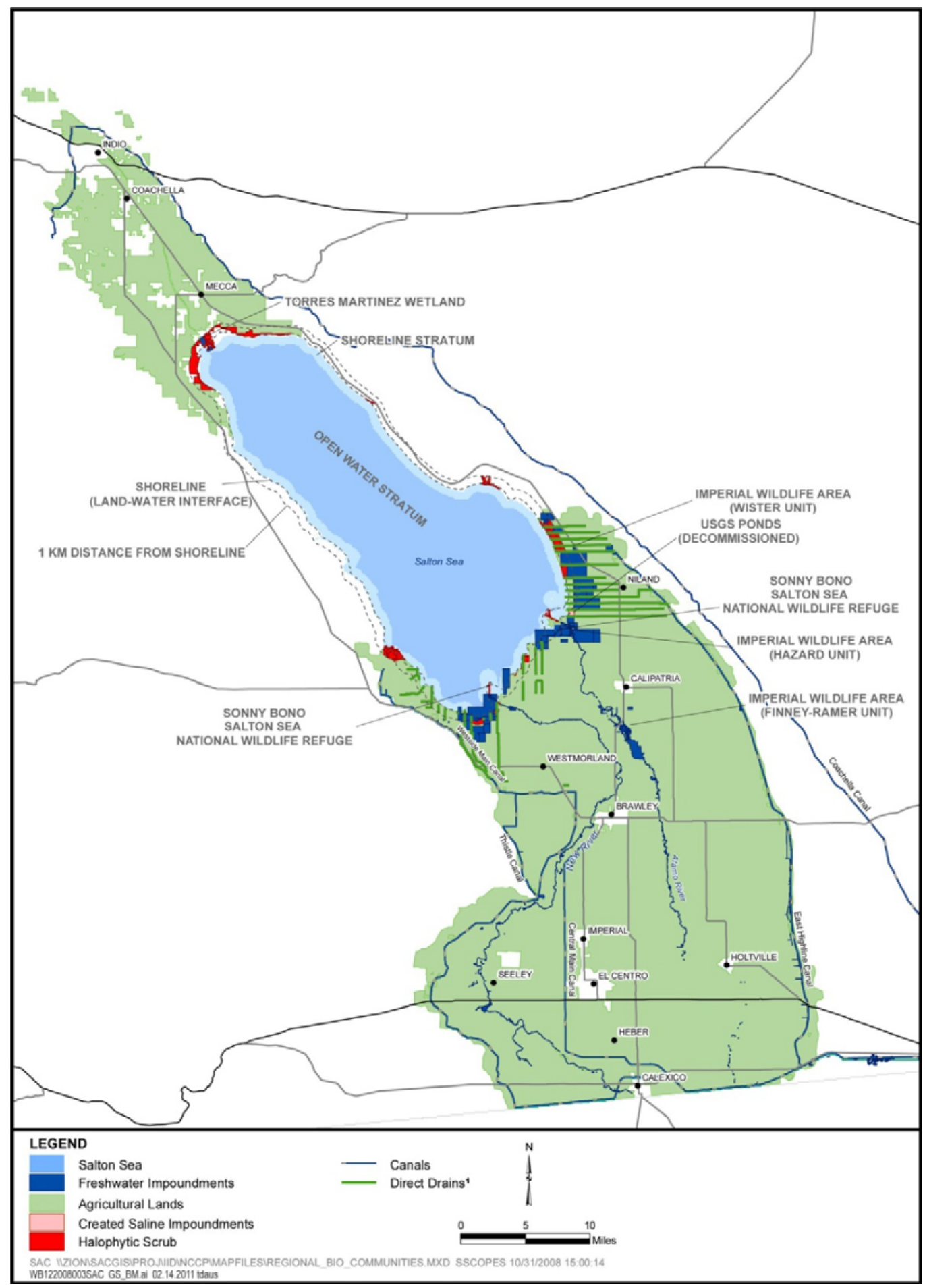

Figure 5. Geographic strata for biological monitoring at the Salton Sea. Base from California Resources Agency, 2006, Salton Sea Ecosystem Restoration Program Draft Programmatic Environmental Impact

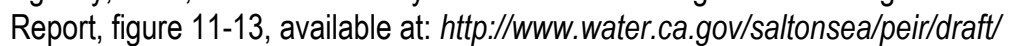

${ }^{1}$ Direct drains in the Coachella Valley at the north end of the Salton Sea are not shown on this figure but are included in the direct drain stratum. 


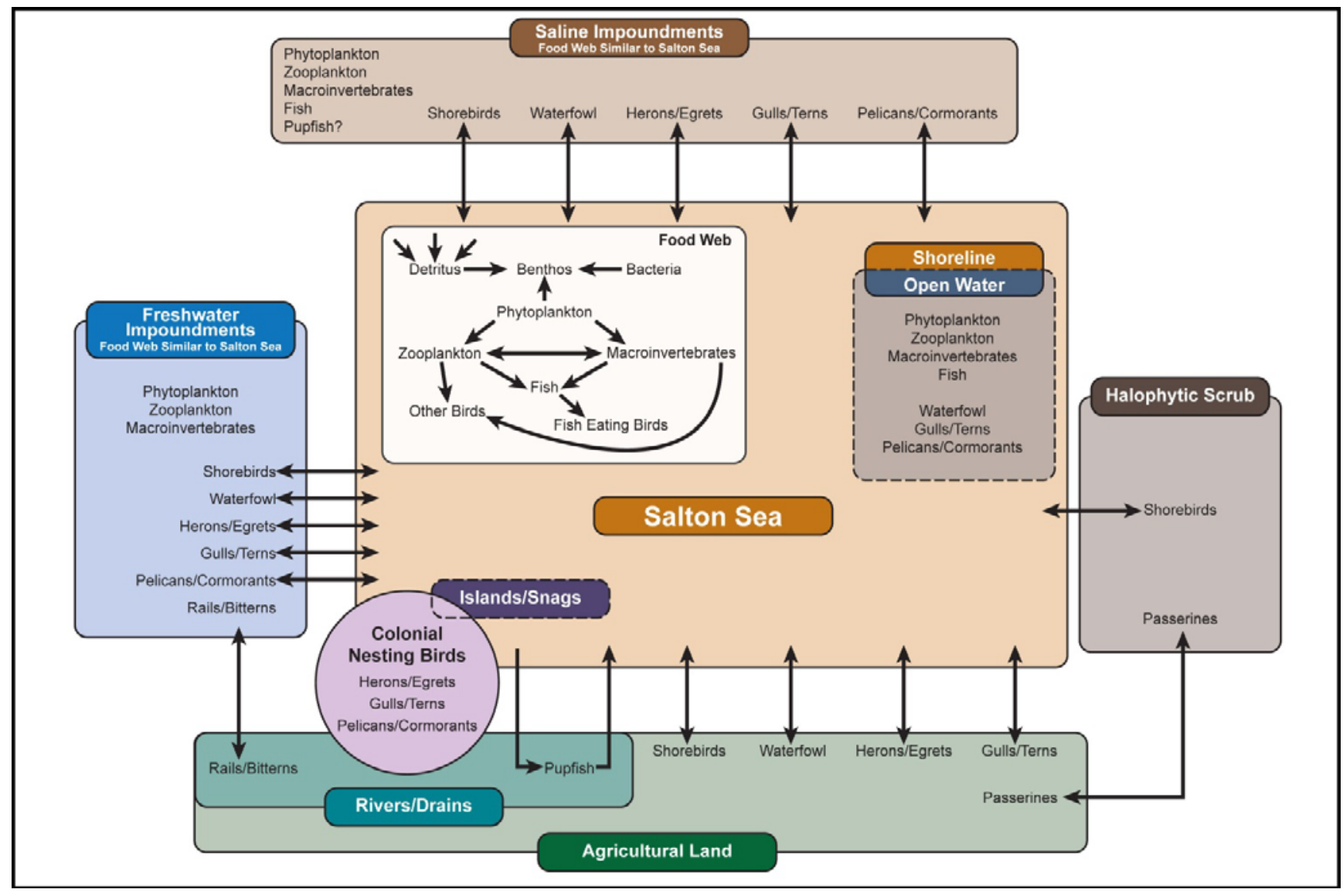

Figure 6. Conceptual model for biological resources at the Salton Sea. 


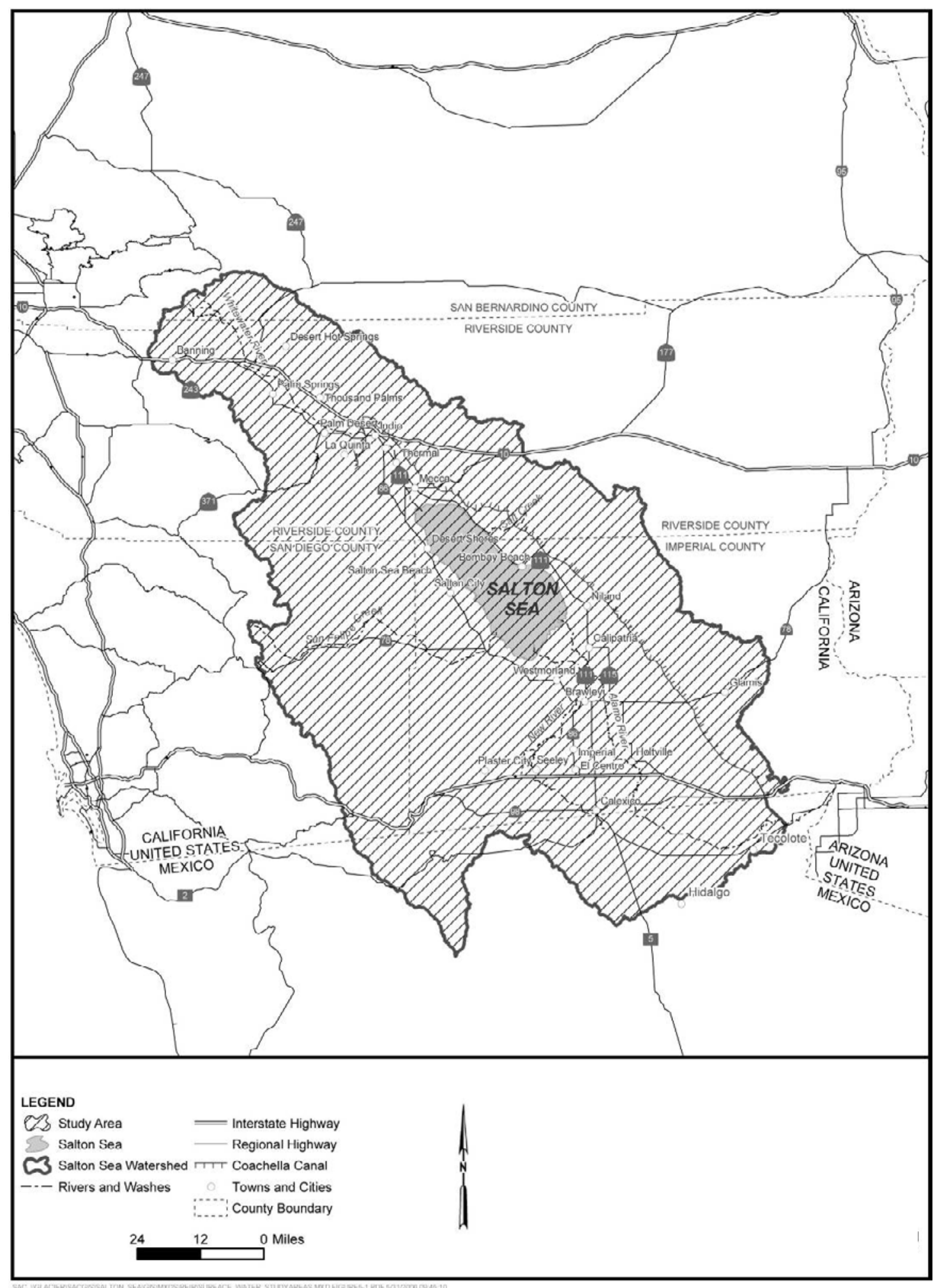

Figure 7. Geographic scope of hydrologic monitoring and assessment. Base from California Resources Agency, 2006, Salton Sea Ecosystem Restoration Program Draft Programmatic Environmental Impact Report, figure 5-1, available online at URL http://www.water.ca.gov/saltonsea/peir/draft/ 


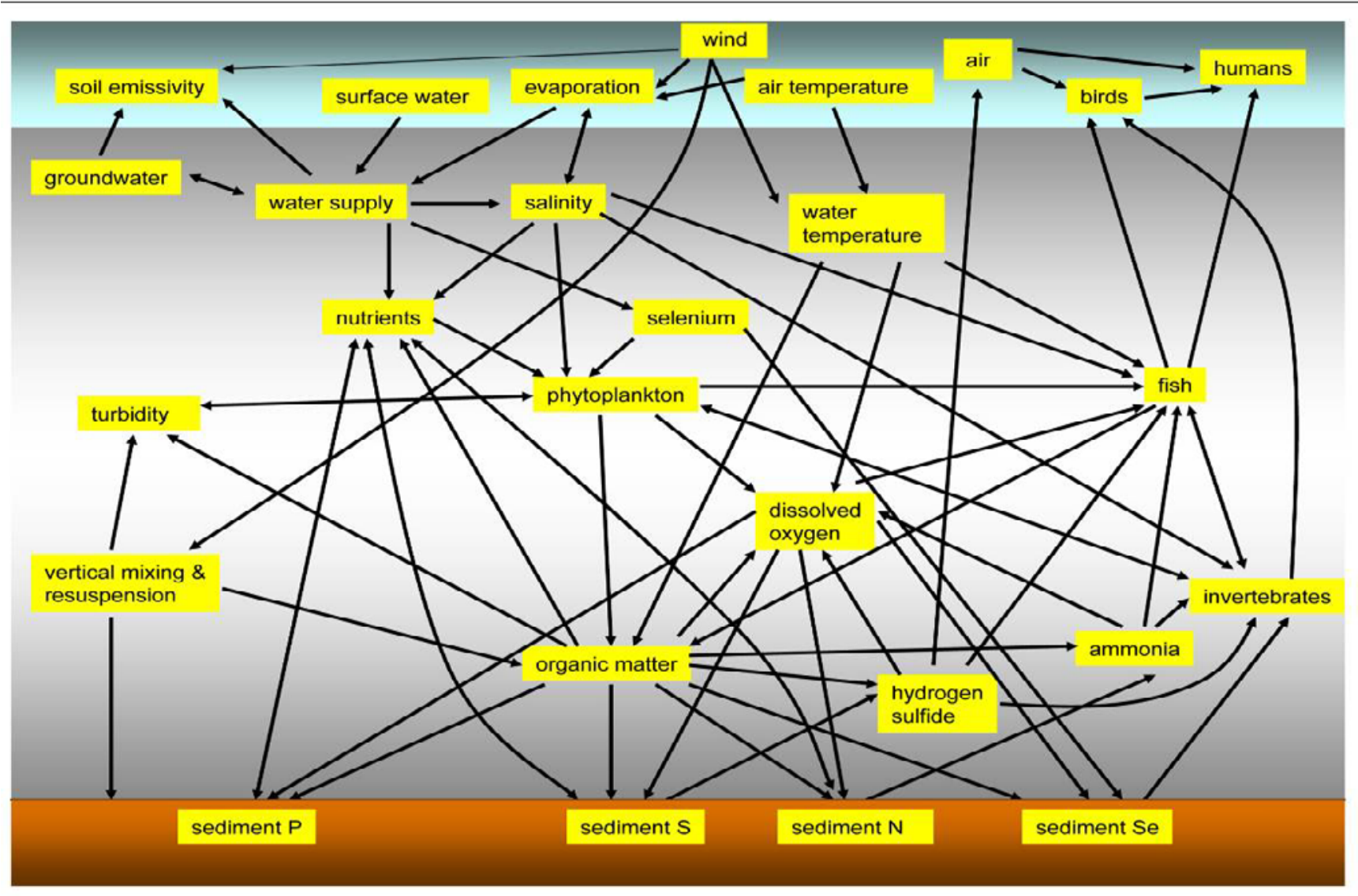

Figure 8. Conceptual model of hydrology at the Salton Sea. 


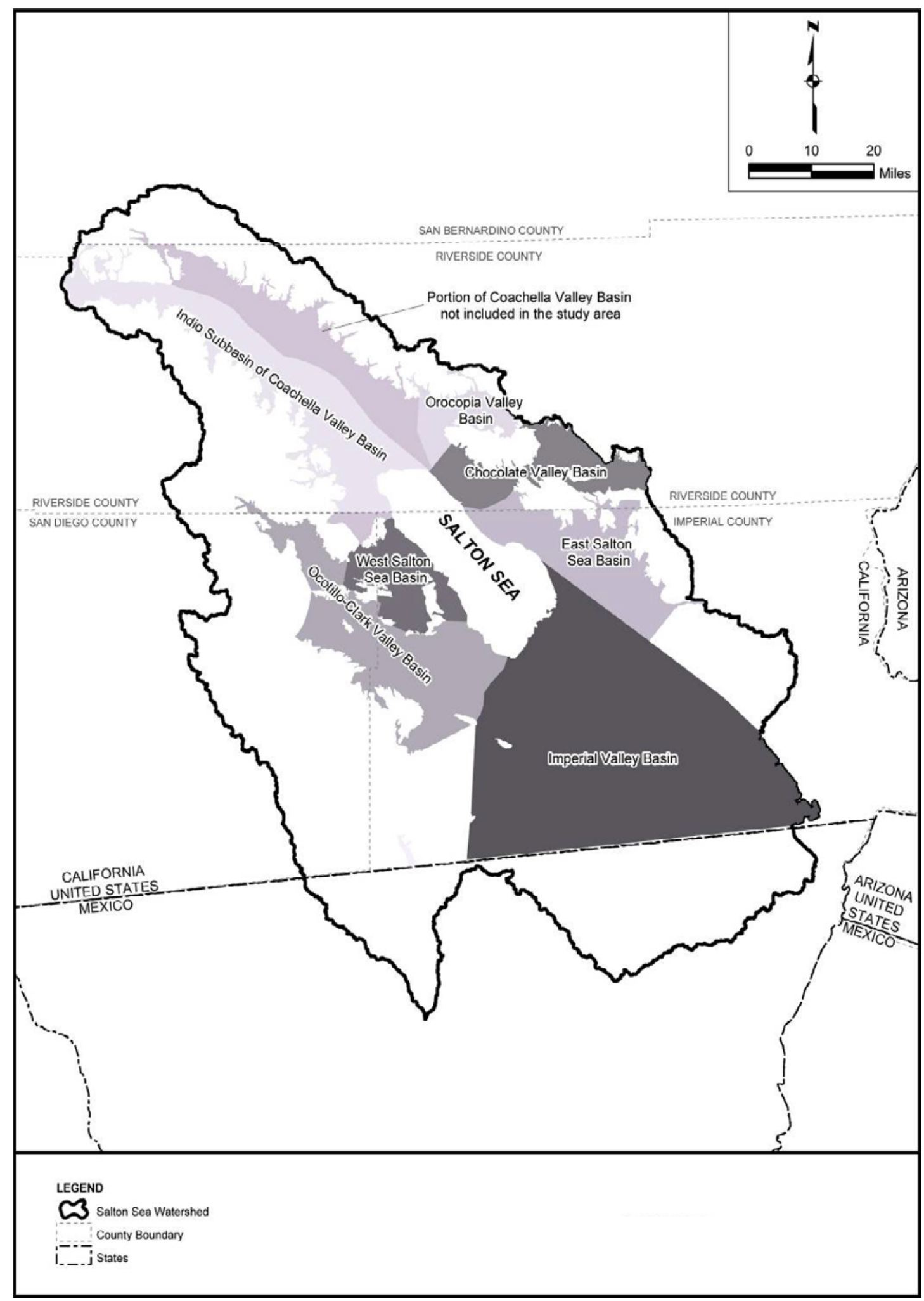

Figure 9. Groundwater basins around the Salton Sea. From California Resources Agency, 2006, Salton Sea Ecosystem Restoration Program Draft Programmatic Environmental Impact Report, figure 7-1, available online at URL http://www.water.ca.gov/saltonsea/peir/draft/ 
62

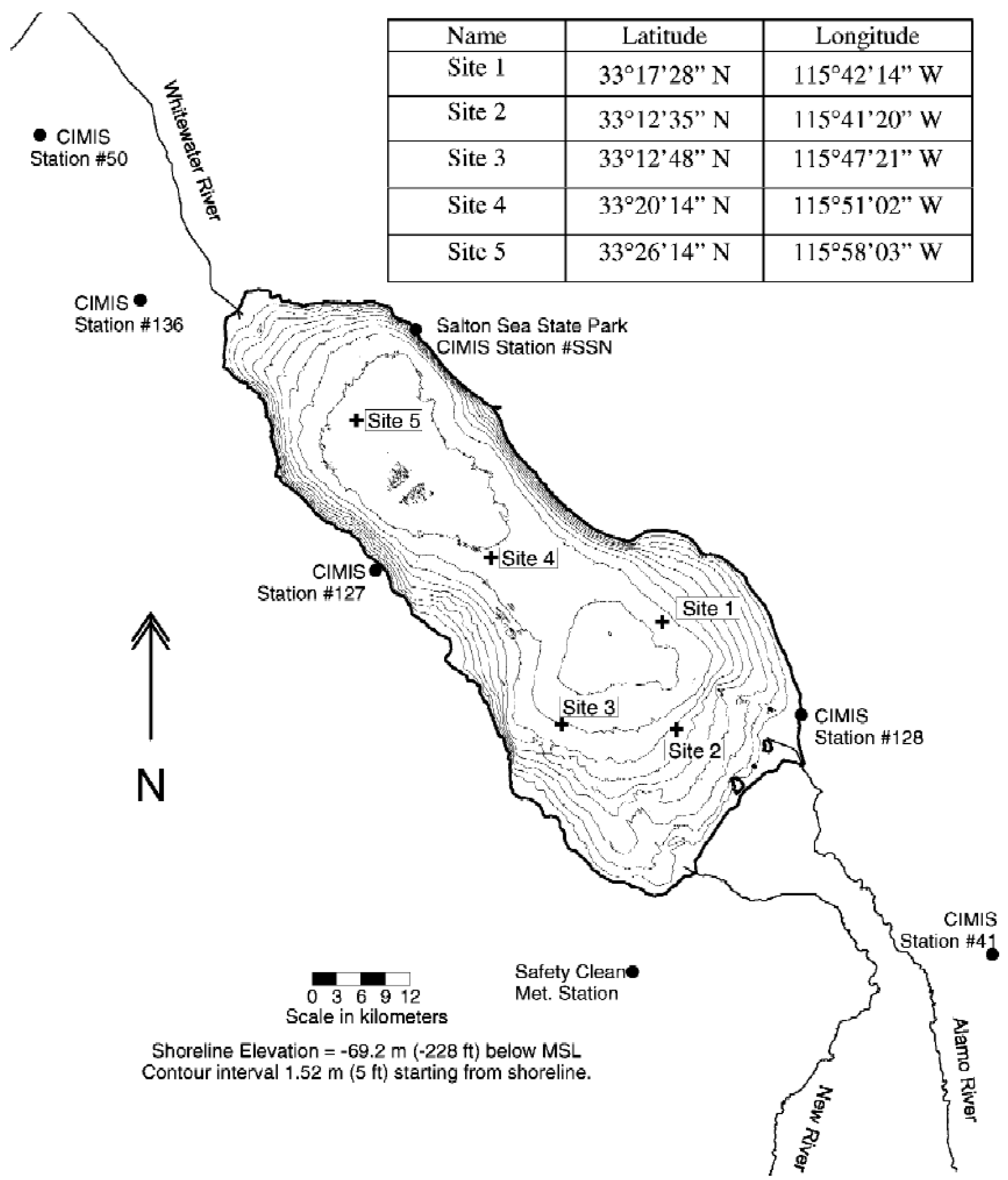

Figure 10. Locations of thermistor and velocity measurement sites. Source: Cook, C.B., Orlob, G.T., and Huston, D.W., 2002, Simulation of wind-driven circulation in the Salton Sea: implications for indigenous ecosystems: Hydrobiologia, v. 473, p. 59-75. 


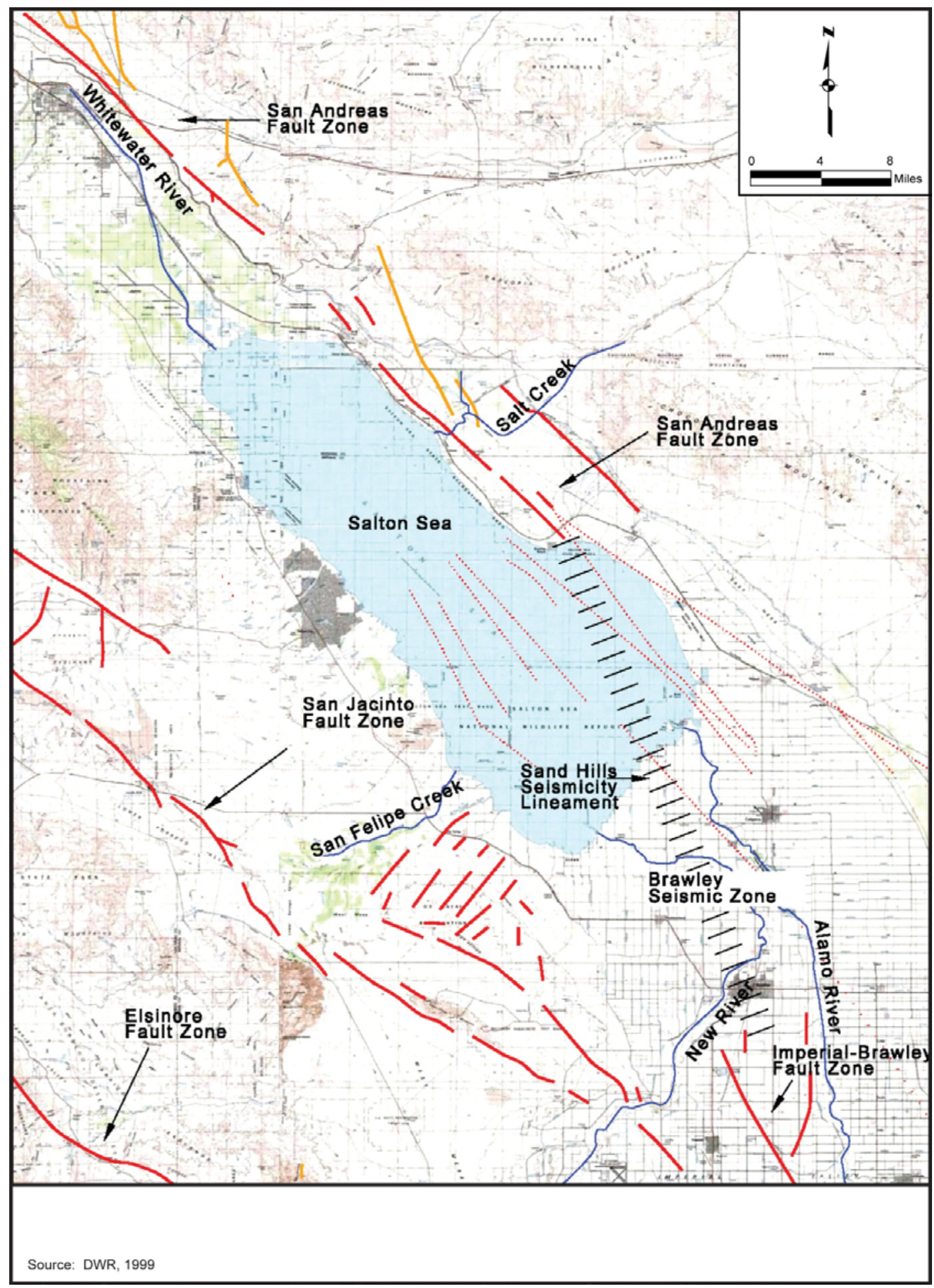

Figure 11. Geographic area for geology and seismicity monitoring. From California Resources Agency, 2006, Salton Sea Ecosystem Restoration Program Draft Programmatic Environmental Impact Report, figure 9-1, available online at URL http://www.water.ca.gov/saltonsea/peir/draft/ 


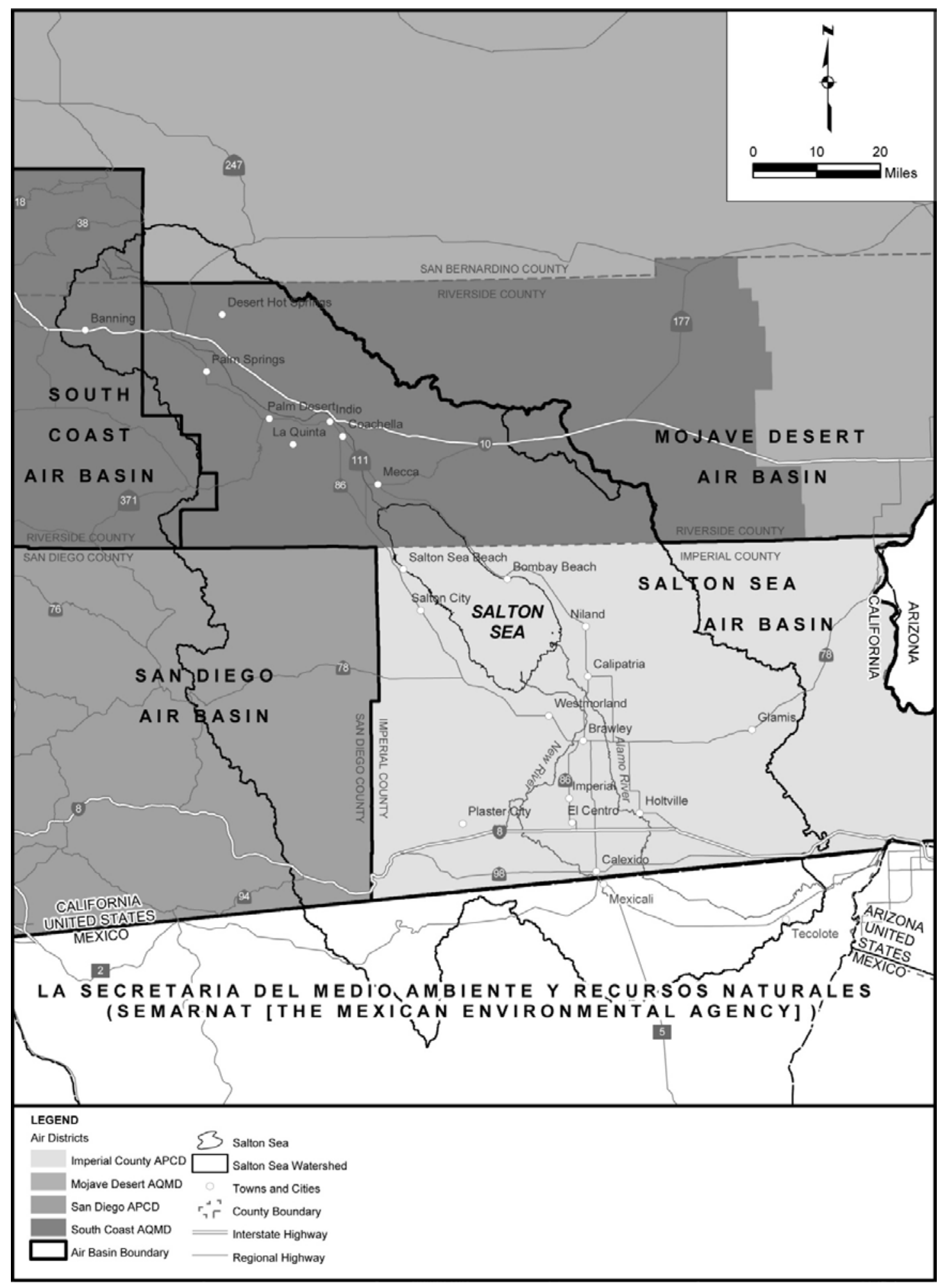

Figure 12. Geographic area for air-quality monitoring. From California Resources Agency, 2006, Salton Sea Ecosystem Restoration Program Draft Programmatic Environmental Impact Report, figure 10-1, available online at URL http://www.water.ca.gov/saltonsea/peir/draft/ 


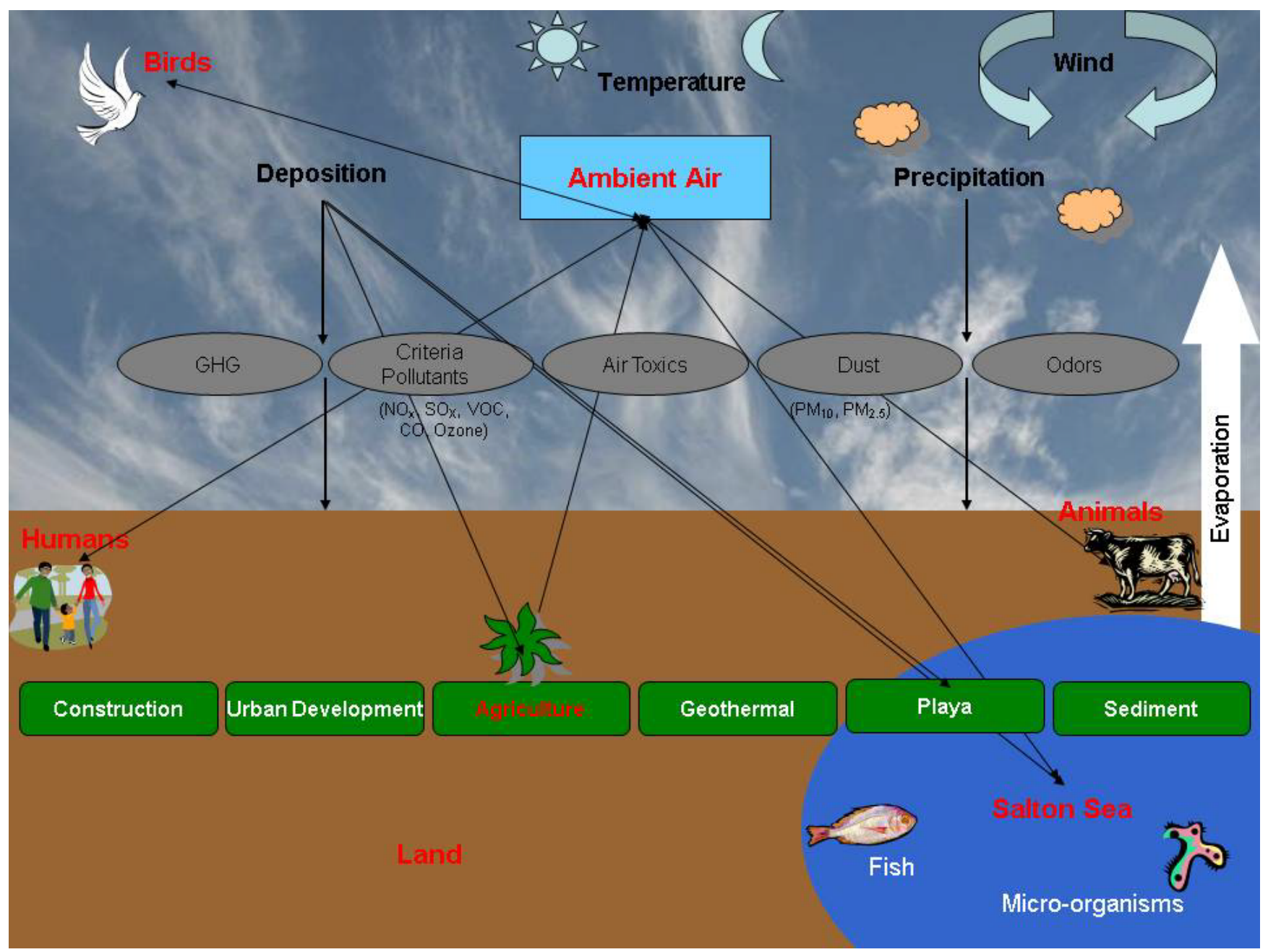

Figure 13. Conceptual model of factors affecting air quality. 


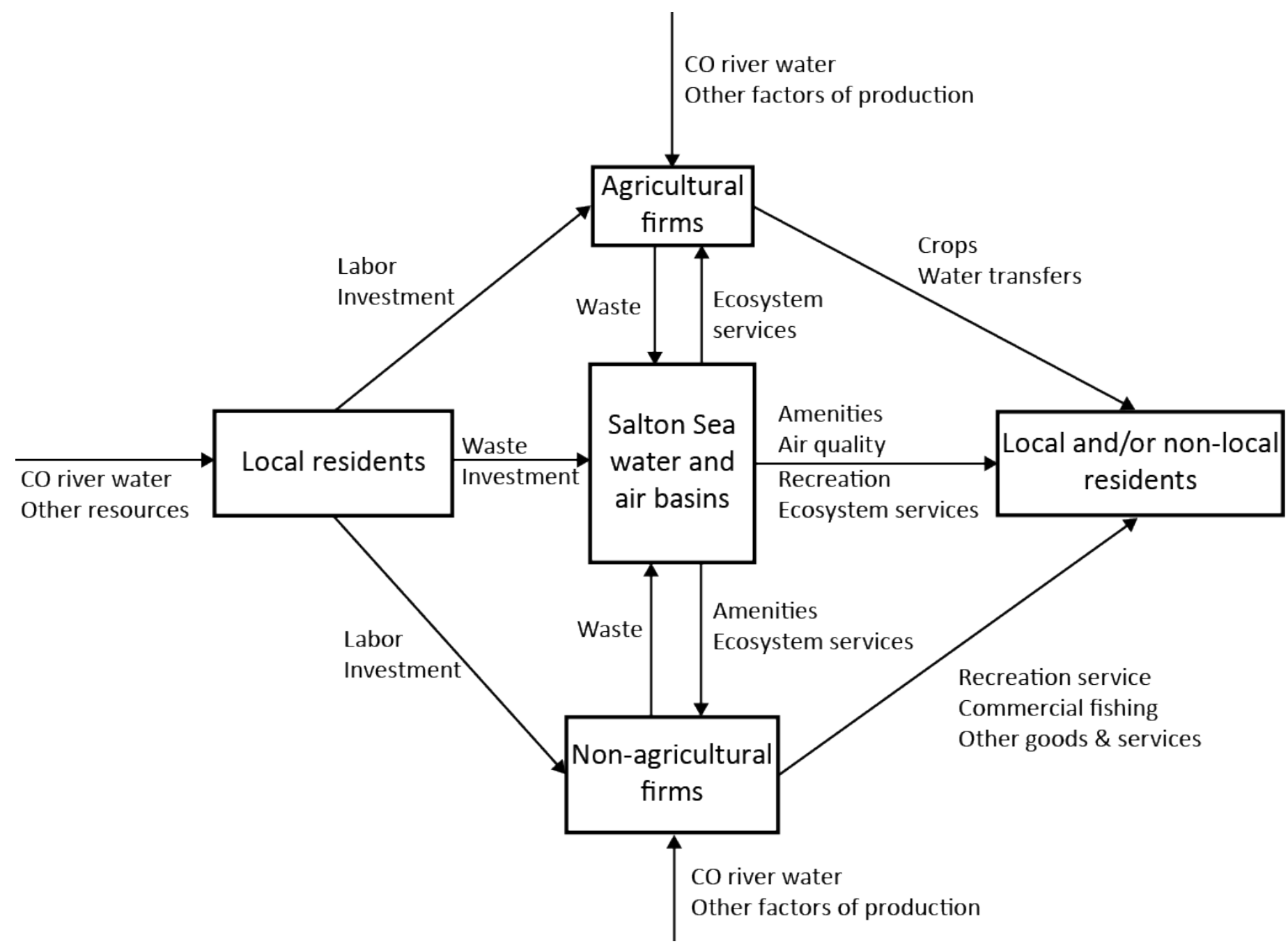

Figure 14. Conceptual model of the Salton Sea socioeconomic system. 


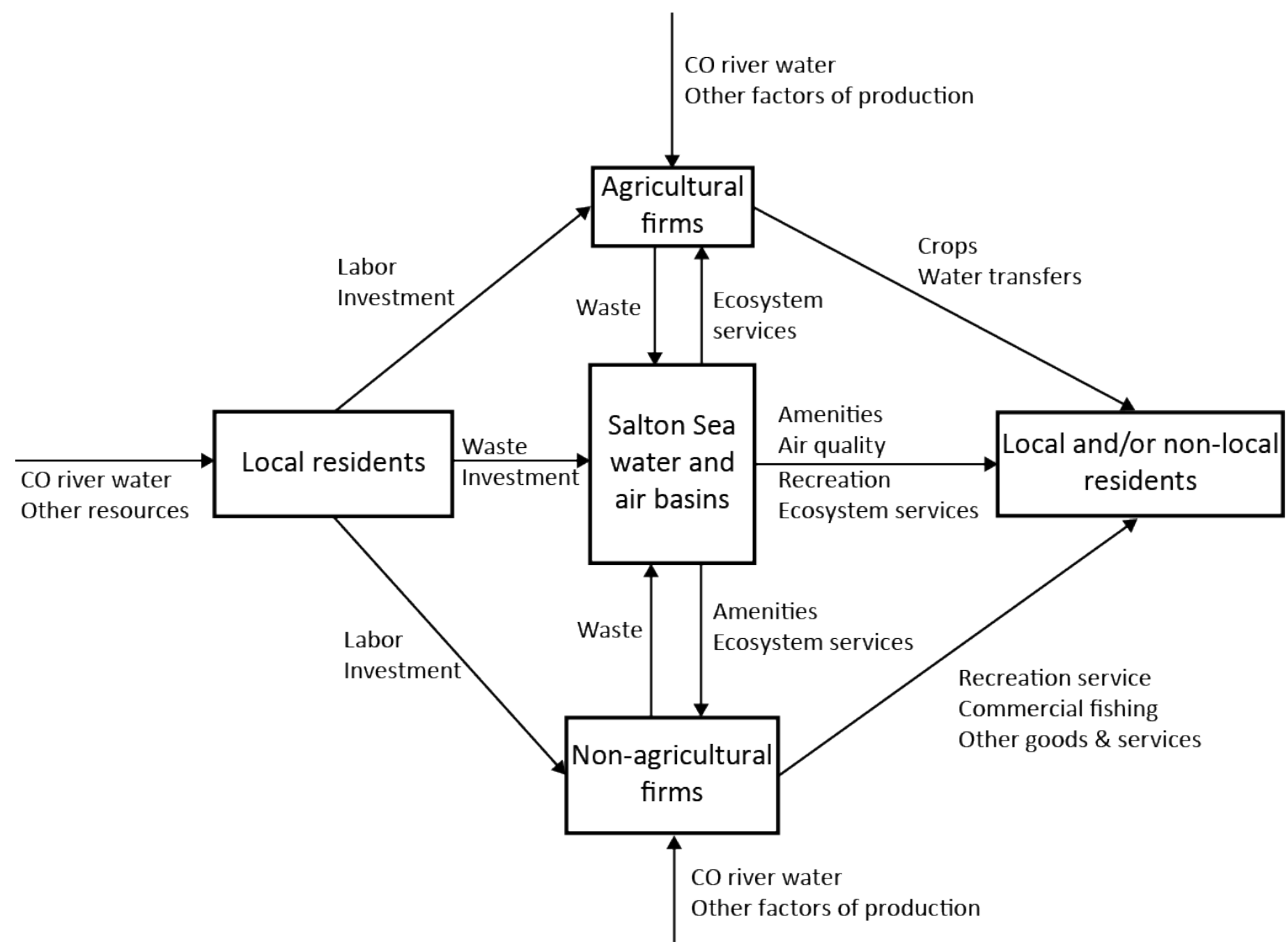

Figure 14. Conceptual model of the Salton Sea socioeconomic system. 
Tables 
Table 1. Salton Sea biological resources monitoring questions and information needs. Question

What are the trends in bird use in the Salton Sea area? Species composition

Seasonal patterns of use

Spatial distribution of use

Relative abundance

What are the environmental attributes and conditions

Water depth

that affect bird use at the Salton Sea?

Substrate characteristics

Thermal stratification

Hydrogen sulfide levels

Salinity

Location, condition, and characteristics of snags, islands, and other roosting/nesting features

Proximity of adjacent agricultural lands

Proximity of adjacent refuges

Availability of food (covered under fish and

invertebrates below)

Location and extent of edge

Proximity to freshwater

Location and characteristics of freshwater (brackish)

inputs

Proximity of potential human activity (disturbance)

What factors contribute to the productivity of breeding birds at the Salton Sea?

What are the trends in fish populations in the Salton Sea area?

What are the environmental attributes and conditions that affect fish populations in the Salton Sea?

What are the trends in desert pupfish populations in the Salton Sea area?
Abundance of colonial breeding waterbirds at the Salton Sea

Seasonal patterns of breeding at the Salton Sea

Characteristics of breeding locations

Breeding success

Species composition and seasonal catch rates in the Salton Sea

Species composition and seasonal catch rates in Salton Sea tributaries, including agricultural drains that discharge directly to the Salton Sea

Water depth

Substrate characteristics

Thermal stratification

Hydrogen sulfide levels

Salinity

River inflow volume and rate

Food availability

Presence/absence (occupancy) trends in the drains discharging directly to the Salton Sea, and shoreline pools, marina areas, and washes and other tributaries 
Table 1. Salton Sea biological resources monitoring questions and information needs.

Question

What are the environmental attributes and conditions that affect desert pupfish fish populations in the Salton Sea area?

What are the trends in benthic and water column macroinvertebrate populations in the Salton Sea and created saline habitats?

What are the environmental attributes and conditions that affect benthic and water column macroinvertebrate populations in the Salton Sea and created saline habitats?

What are the trends of plankton populations in the Salton Sea and created saline habitats?

What are the environmental attributes and conditions that affect plankton populations in the Salton Sea and created saline habitats?
Water depth

Information Needs

Water velocity in drains used by desert pupfish

Substrate characteristics

Vegetation/cover characteristics (in drains and created saline habitats)

Fish, invertebrate, reptile, and amphibian community composition associated with desert pupfish use Food availability

Species composition and density (\#/unit volume) in the Salton Sea and created saline habitats

Seasonal patterns in density

Spatial distribution of species

Water depth

Substrate characteristics

Thermal stratification

Hydrogen sulfide levels

Salinity

Species composition and density (\#/unit volume) in the Salton Sea and created saline habitats

Seasonal patterns in density

Spatial distribution of species

Water depth

Substrate characteristics

Thermal stratification

Hydrogen sulfide levels

Salinity 
Table 2. Biological resource monitoring activities and geographic strata in which they will be performed.

\begin{tabular}{|c|c|c|c|c|c|c|c|}
\hline \multirow[b]{2}{*}{ Monitoring Activity } & \multicolumn{7}{|c|}{ Geographic Strata } \\
\hline & 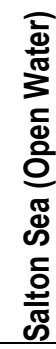 & 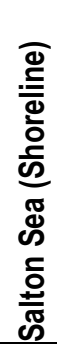 & 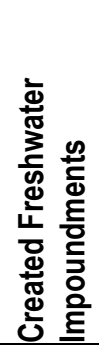 & 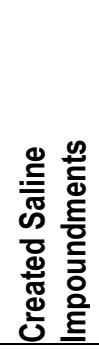 & 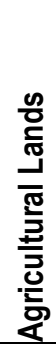 & 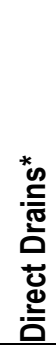 & 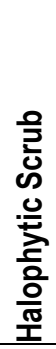 \\
\hline Bird Surveys & $\mathrm{X}$ & $\mathrm{X}$ & $\mathrm{X}$ & $\mathrm{X}$ & $\mathrm{X}$ & & $\mathrm{X}$ \\
\hline Colonial Breeding Bird Surveys & & $\mathrm{X}$ & & & & & $\mathrm{X}$ \\
\hline Bird Counts at Roost Sites & & $\mathrm{X}$ & $\mathrm{X}$ & & $\mathrm{X}$ & & $\mathrm{X}$ \\
\hline Dead and Sick Bird Counts & $\mathrm{X}$ & $\mathrm{X}$ & $\mathrm{X}$ & $\mathrm{X}$ & & & \\
\hline Fish Surveys & $\mathrm{X}$ & $\mathrm{X}$ & $\mathrm{X}$ & $\mathrm{X}$ & & & \\
\hline Desert Pupfish Surveys & & $\mathrm{X}$ & $X$ & $x$ & & $\mathrm{X}$ & \\
\hline Water Column Phytoplankton Surveys & $\mathrm{X}$ & $\mathrm{X}$ & $\mathrm{X}$ & $\mathrm{X}$ & & & \\
\hline Water Column Zooplankton Surveys & $\mathrm{X}$ & $\mathrm{X}$ & $\mathrm{X}$ & $\mathrm{X}$ & & & \\
\hline Water Column Macroinvertebrate Surveys & $\mathrm{X}$ & $\mathrm{X}$ & $\mathrm{X}$ & $\mathrm{X}$ & & & \\
\hline Benthic Macroinvertebrate Surveys & $\mathrm{X}$ & $\mathrm{X}$ & $\mathrm{X}$ & $\mathrm{X}$ & & & \\
\hline Vector (mosquito) Monitoring & & $\mathrm{X}$ & $\mathrm{X}$ & $\mathrm{X}$ & & & \\
\hline
\end{tabular}

*Bird surveys performed within the Agricultural Lands stratum will include the Direct Drain stratum. 
Table 3. Objectives and purpose of the avian monitoring activities.

Objective Purpose

Establish existing abundance of selected species or groups of species at a level of precision that is meaningful and at temporal and spatial scales that adequately capture the range of variability.

Monitor change in abundance of selected species or groups of species over time.

Document current abundance of birds at the Salton Sea

Provide basis for evaluating change

Provide basis for comparison to species abundance elsewhere to gain a better understanding of the value of the Salton Sea

Provide basis for comparison to historic conditions Provide basis for evaluating performance of potential future restoration activities Support potential environmental documentation associated with restoration

Document trends in abundance or occupancy by birds

Provide basis for determining linkages to changes in abundance elsewhere

Provide basis for identifying correlations between changes in bird use and changes in environmental conditions at the Salton Sea

Provide basis for evaluating response of birds to potential future restoration activities Support potential environmental documentation associated with restoration

Identify potential relationships between bird use and environmental attributes.

Provide basis for initially identifying relationships between bird use and environmental attributes Provide technical basis for planning, designing, and implementing focused investigations Provide technical basis for locating, planning, designing, and managing potential created habitats Provide technical basis for managing adjacent habitat areas Support potential environmental documentation associated with restoration 
Table 4. Objectives and purpose of the fish monitoring activities.

\section{Objective}

Establish existing conditions for levels of fish catches (catch per unit effort) within the Salton Sea and its tributaries at appropriate temporal and spatial scales.

Establish existing conditions for levels of habitat occupancy by desert pupfish within Salton Sea and its tributaries at appropriate temporal and spatial scales.

Establish existing conditions for levels of fish abundance within created saline habitats at appropriate temporal and spatial scales.

Monitor change in catch per unit effort, percent occupancy, and abundance over time, as appropriate.

Identify potential relationships between fish use and environmental attributes.
Document current catch per unit effort for fish in the Salton Sea

Provide basis for evaluating change

Provide basis for evaluating performance of potential future restoration activities

Support potential environmental documentation associated with restoration

Provide the basis for determining percent occupancy by desert pupfish in Salton Sea tributaries

Provide basis for evaluating change

Provide basis for evaluating performance of potential future restoration activities

Support potential environmental documentation associated with restoration

Document current abundance of fish within existing created saline habitats

Provide basis for evaluating change in abundance

Provide information that would contribute to identifying important environmental attributes Support potential environmental documentation associated with restoration

Document trends in catch per unit effort, abundance, or occupancy by fish in the Salton Sea area over time Provide information that will contribute to identifying correlations between changes in fish populations and changes in environmental conditions at the Salton Sea Provide basis for evaluating response of fish-eating birds to changes in the fish population Provide information that will support the design and management of habitats created to support fish and birds Support potential environmental documentation associated with restoration

Provide basis for initially identifying relationships between fish populations and environmental attributes Provide technical basis for planning, designing, and implementing focused investigations Provide technical basis for locating, planning, designing, and managing potential created habitats to support fish and fish-eating birds

Provide technical basis for managing adjacent habitat areas (for example, tributaries)

Support potential environmental documentation associated with restoration 
Table 5. Objectives and purpose of the invertebrate monitoring activities.

\section{Objective}

Establish existing conditions for selected plankton and invertebrate densities within the Salton Sea and created saline impoundments at appropriate temporal and spatial scales.

Monitor change in plankton and macroinvertebrate densities over time.

Identify potential relationships between measures of plankton and invertebrate abundance and environmental attributes.
Purpose

Document species composition and density of selected plankton and macroinvertebrates in the Salton Sea and created saline habitats

Provide basis for evaluating change

Provide basis for evaluating performance of potential future restoration activities

Support potential environmental documentation associated with restoration

Document trends in plankton and macroinvertebrate densities in the Salton Sea and created saline impoundments over time

Provide information that will contribute to identifying correlations between changes in the density of these organisms and changes in environmental conditions at the Salton Sea and created saline impoundments

Provide information that will support the design and management of habitats created to support fish and birds

Support potential environmental documentation associated with restoration

Provide basis for initially identifying relationships between plankton and invertebrate populations and environmental attributes

Provide technical basis for planning, designing, and implementing focused investigations

Provide technical basis for locating, planning, designing, and managing potential created habitats to support fish and birds

Provide technical basis for managing adjacent habitat areas (for example, tributaries)

Support potential environmental documentation associated with restoration 
Table 6. Inflow contributions to the Salton Sea. Inflow Source to the Salton Sea

Percent of Historical Annual Average Inflow*

\begin{tabular}{lc} 
Mexico & 9.8 \\
Imperial Valley & 76.5 \\
Coachella Valley & 8.5 \\
Local Watershed & 1.5 \\
Precipitation directly on the Salton Sea & 3.7 \\
TOTAL & 100.0 \\
\hline
\end{tabular}

Source: Resources Agency (2006). Period 1950-2002, App H-2 Table H2-2

*Does not include groundwater, which accounts for only about $0.01 \%$ of the total annual inflow. 
Table 7. Salton Sea hydrology monitoring questions and information needs.

\section{Question}

What are the trends in inflow/outflow quantity?

What factors affect inflow/outflow quantity?

What are the trends in water quality of surface inflows and sediment (rivers, drains, creeks)?

What are the trends in Salton Sea water and sediment quality?

What is the risk associated with selenium in the Salton Sea?

How does groundwater interact with the Salton Sea and exposed playa?

How do physical and biological factors influence the hydrodynamics in the Salton Sea?
Information Needs

Inflow amount (temporal and spatial)

Water-surface elevation/area

Evaporation rates (temporal) ${ }^{*}$

Salinity (affects evaporation)

Precipitation amount ${ }^{*}$

Concentrations of conventional water quality constituents (temporal and spatial)

Concentrations of nutrients (temporal and spatial)

Concentration of selenium (temporal and spatial)

Concentrations of conventional water quality constituents (temporal and spatial)

Concentrations of nutrients (temporal and spatial)

Concentration of selenium (temporal and spatial)

Timing, frequency, and duration of episodic events

Selenium concentrations in inflow water and sediment Selenium concentrations in Salton Sea water, sediment, invertebrates, and fish

Shallow groundwater depth and quality in areas of exposed playa (temporal and spatial)

Shallow groundwater depth and quality in areas of potential saline habitat

Water-surface elevation (Salton Sea)

Wind speed and direction ${ }^{*}$

Solar radiation *

Air temperature *

Humidity ${ }^{*}$

Water temperature (profile)

Direction and speed of currents

Suspension *

Pycnocline (spatial extent, duration, biological

productivity)

Decomposition *

Sinks*

Volatization ${ }^{*}$

Elevation

Bathymetry*

"Data that will be collected as part of monitoring for other resource areas or future focused investigation. 
Table 8. Objectives and purpose of the surface water monitoring activities. Objective Measure surface water flow levels to the Salton Sea.

Document current annual input volume to the Salton Sea (surface inflow component of the water budget) Document seasonal patterns of inflow Document distribution of inflow from various tributaries

Support potential future environmental documentation associated with restoration

Monitor changes in surface water flows to the Salton Sea over time.

Document trends in surface water inflow to the Salton Sea

Provide basis for projecting future inflow conditions and estimating the reliability of water supply for potential future created habitats and air quality mitigation

Provide information that will clarify linkages between surface water flows and land use activities Contribute to understanding the hydrodynamics of the Salton Sea

Provide information that will support the design and management of created habitats

Support potential environmental documentation associated with restoration

Measure water-surface elevation in the Salton Sea.

Document current annual (average) water-surface elevation in the Salton Sea

Document seasonal patterns in water-surface elevation Contribute to projecting future volume, area, and depth of the Salton Sea

Support potential environmental documentation associated with restoration 
Table 9. Flow monitoring gages in the Salton Sea watershed.

\begin{tabular}{llll}
\multicolumn{1}{c}{ Source } & \multicolumn{1}{c}{ Location } & \multicolumn{1}{c}{ Agency } & Frequency \\
\hline Alamo River & International Boundary & IID, IBWC & Continuous \\
Alamo River & near Niland & USGS 10254730 & Continuous \\
New River & International Boundary & USGS 10254970 & Continuous \\
New River & near Westmorland & USGS 10255550 & Continuous \\
Whitewater & near Mecca & USGS 10259540 & Continuous \\
River/CVSC & & & \\
Whitewater River & near Indio & USGS 10259300 & Continuous \\
Salt Creek & 0.3 mi upland of Salton & USGS 10255550 & Continuous \\
& Sea & & \\
San Felipe Creek & near Westmorland & USGS 10255885 & Discontinued \\
\hline
\end{tabular}


Table 10. Objectives and purpose of shallow groundwater monitoring activities.

Objective

Measure shallow groundwater levels in the Salton Sea area.

Monitor change in shallow groundwater levels in the Salton Sea area over time.

Measure quality of shallow groundwater near the Salton Sea

Monitor change in shallow groundwater quality in the Salton Sea area over time.
Document current shallow groundwater levels Document seasonal changes in shallow groundwater levels

Support potential future environmental documentation associated with restoration

Document trends in shallow groundwater levels in the Salton Sea area

Provide basis for identifying factors that influence shallow groundwater level (for example, Salton Sea elevation)

Provide basis for projecting shallow groundwater depth at potential restoration or air quality management sites on exposed Salton Sea playa Provide information that will support the design and management of created habitats

Support potential environmental documentation associated with restoration

Document current shallow groundwater quality Document seasonal changes in shallow groundwater quality

Support potential future environmental documentation associated with restoration

Document trends in shallow groundwater quality in the Salton Sea area

Provide basis for identifying factors that influence shallow groundwater quality (for example, linkage to surface water quality)

Provide information that will support the design and management of created habitats and air quality management sites Support potential environmental documentation associated with restoration 
Table 11. Objectives and purpose of hydrodynamics (water temperature and current) monitoring activities.

\begin{tabular}{|c|c|}
\hline Objective & $\begin{array}{l}\text { Purpose } \\
\end{array}$ \\
\hline Measure water temperature profiles of the Salton Sea. & $\begin{array}{l}\text { Document current water temperature profile in the } \\
\text { Salton Sea } \\
\text { Document depth and strength of density stratification } \\
\text { Document seasonal changes in the water temperature } \\
\text { profile } \\
\text { Support potential future environmental documentation } \\
\text { associated with restoration }\end{array}$ \\
\hline $\begin{array}{l}\text { Monitor change in water temperature profiles in the } \\
\text { Salton Sea over time. }\end{array}$ & $\begin{array}{l}\text { Document trends in water temperature in the Salton } \\
\text { Sea } \\
\text { Provide basis for clarifying factors that influence } \\
\text { water temperatures in the Salton Sea (for example, } \\
\text { wind-driven turnover events) } \\
\text { Provide basis for projecting water temperature profiles } \\
\text { (and stratification) under different water body } \\
\text { configurations and depths } \\
\text { Contribute to understanding hydrodynamic } \\
\text { relationships (for example, nutrient cycling) in the } \\
\text { Salton Sea } \\
\text { Provide information that will support the design and } \\
\text { management of created habitats } \\
\text { Support potential environmental documentation } \\
\text { associated with restoration }\end{array}$ \\
\hline Measure water current patterns in the Salton Sea & $\begin{array}{l}\text { Document existing current patterns in the Salton Sea } \\
\text { Document seasonal changes in current patterns } \\
\text { Support potential future environmental documentation } \\
\text { associated with restoration }\end{array}$ \\
\hline $\begin{array}{l}\text { Monitor change in water current patterns in the Salton } \\
\text { Sea over time. }\end{array}$ & $\begin{array}{l}\text { Document trends in water current patterns in the } \\
\text { Salton Sea } \\
\text { Provide basis for clarifying factors that influence } \\
\text { water currents (for example, prevailing winds) } \\
\text { Contribute to understanding hydrodynamic } \\
\text { relationships (for example, nutrient cycling) in the } \\
\text { Salton Sea } \\
\text { Provide information that will support the design and } \\
\text { management of created water bodies } \\
\text { Support potential environmental documentation } \\
\text { associated with restoration }\end{array}$ \\
\hline
\end{tabular}


Table 12. Objectives and purpose of water and sediment quality monitoring activities.

Objective Purpose

Measure water and sediment quality in the Salton Sea

Document current water and sediment quality in the

area.

Salton Sea area (including Salton Sea and tributaries)

Support identification and evaluation of potential

environmental and human risks associated with water

or sediment-borne compounds

Provide information that will support the design and

management of created habitats

Support potential future environmental documentation associated with restoration

Monitor change in water and sediment quality in the

Document trends in water and sediment quality in the

Salton Sea area over time.

Salton Sea area

Support identification and evaluation of potential environmental and human health risks associated with water or sediment-borne compounds

Provide basis for identifying factors that influence water and sediment quality in the Salton Sea (for example, wind-driven turnover events)

Provide basis for projecting future water quality characteristics in the Salton Sea and potential created water bodies (for example, water quality modeling) Contribute to understanding hydrodynamic relationships (for example, nutrient cycling) in the Salton Sea

Provide information that will support the design and management of created habitats

Support potential environmental documentation associated with restoration 
Table 13. Location and sampling frequency for water and sediment quality parameters.

\section{Location}

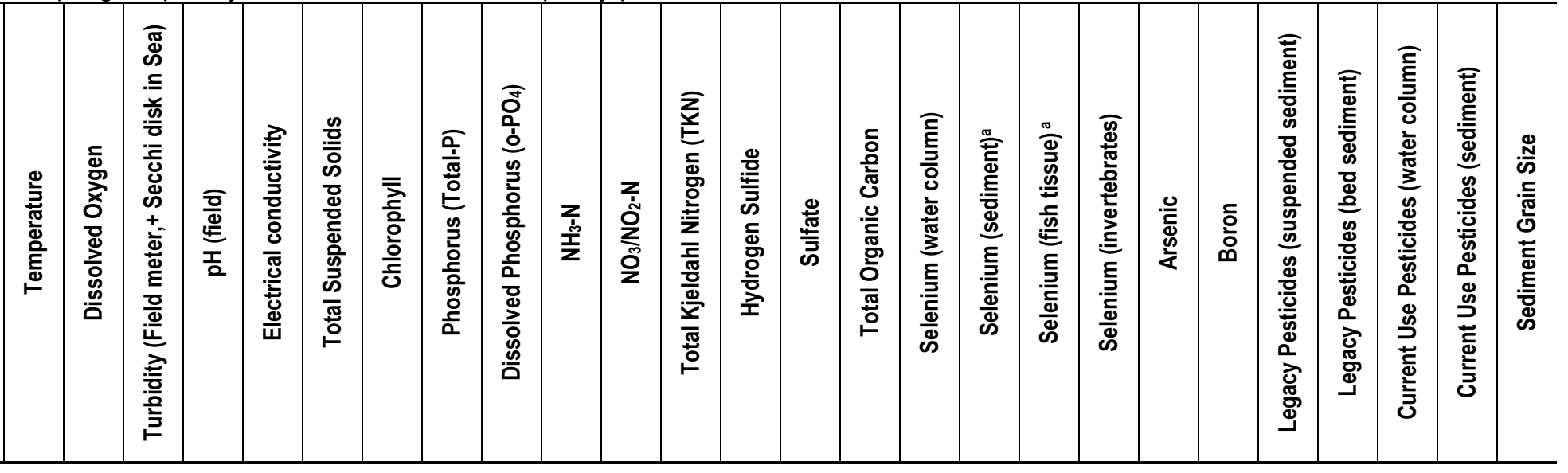

Inflows - Surface Water and Sediment

\begin{tabular}{|c|c|c|c|c|c|c|c|c|c|c|c|c|c|c|c|c|c|c|c|c|c|c|c|c|c|c|}
\hline $\begin{array}{l}\text { New River at International } \\
\text { Boundary }\end{array}$ & $\mathrm{C}$ & $\mathrm{C}$ & M & $\mathrm{M}$ & $\mathrm{C}$ & M & - & M & M & M & M & M & - & M & - & $\mathrm{M}$ & - & - & - & M & M & A & A & $\mathrm{S}$ & $\mathrm{S}$ & M \\
\hline New River at Outlet & $\mathrm{C}$ & $\mathrm{C}$ & M & $\mathrm{M}$ & $\mathrm{C}$ & $\mathrm{M}$ & - & $\mathrm{M}$ & $\mathrm{M}$ & M & $\mathrm{M}$ & $\mathrm{M}$ & - & $\mathrm{M}$ & - & M & - & A & - & $\mathrm{M}$ & $\mathrm{M}$ & A & A & $\mathrm{S}$ & $\mathrm{S}$ & $\mathrm{M}$ \\
\hline $\begin{array}{l}\text { Alamo River at International } \\
\text { Boundary }\end{array}$ & $\mathrm{C}$ & $\mathrm{C}$ & M & M & $\mathrm{C}$ & M & - & $\mathrm{M}$ & M & M & M & M & - & M & - & $\mathrm{M}$ & - & - & - & M & $\mathrm{M}$ & A & A & $\mathrm{S}$ & $\mathrm{S}$ & M \\
\hline Alamo River at Outlet & $\mathrm{C}$ & $\mathrm{C}$ & $\mathrm{M}$ & $\mathrm{M}$ & $\mathrm{C}$ & $\mathrm{M}$ & - & $\mathrm{M}$ & $\mathrm{M}$ & $\mathrm{M}$ & $\mathrm{M}$ & $\mathrm{M}$ & - & $\mathrm{M}$ & - & $\mathrm{M}$ & - & $\mathrm{A}$ & - & $\mathrm{M}$ & $\mathrm{M}$ & $\mathrm{A}$ & $\mathrm{A}$ & $\mathrm{S}$ & $\mathrm{S}$ & $\mathrm{M}$ \\
\hline Whitewater River at Outlet & $\mathrm{C}$ & $\mathrm{C}$ & $\mathrm{M}$ & $\mathrm{M}$ & $\mathrm{C}$ & $\mathrm{M}$ & - & $\mathrm{M}$ & $\mathrm{M}$ & $\mathrm{M}$ & $\mathrm{M}$ & $\mathrm{M}$ & - & $\mathrm{M}$ & - & $\mathrm{M}$ & - & $\mathrm{A}$ & - & $\mathrm{M}$ & $\mathrm{M}$ & $\mathrm{A}$ & $\mathrm{A}$ & $\mathrm{S}$ & $\mathrm{S}$ & $\mathrm{M}$ \\
\hline Salt Creek & $\mathrm{C}$ & $\mathrm{C}$ & $\mathrm{M}$ & $\mathrm{M}$ & $\mathrm{C}$ & $\mathrm{M}$ & - & $\mathrm{M}$ & $\mathrm{M}$ & $\mathrm{M}$ & $\mathrm{M}$ & $\mathrm{M}$ & - & $\mathrm{M}$ & - & $\mathrm{M}$ & - & - & - & $\mathrm{M}$ & $\mathrm{M}$ & - & - & - & - & $\mathrm{M}$ \\
\hline San Felipe Creek & $\mathrm{C}$ & $\mathrm{C}$ & $\mathrm{M}$ & $\mathrm{M}$ & $\mathrm{C}$ & $\mathrm{M}$ & - & $\mathrm{M}$ & $\mathrm{M}$ & $\mathrm{M}$ & $\mathrm{M}$ & $\mathrm{M}$ & - & $\mathrm{M}$ & - & $\mathrm{M}$ & - & - & - & $\mathrm{M}$ & $\mathrm{M}$ & - & - & - & - & $\mathrm{M}$ \\
\hline Direct Drains & - & $\mathrm{M}$ & $\mathrm{M}$ & $\mathrm{M}$ & $\mathrm{M}$ & $\mathrm{M}$ & - & $\mathrm{M}$ & $\mathrm{M}$ & $\mathrm{M}$ & $\mathrm{M}$ & $\mathrm{M}$ & - & $\mathrm{M}$ & - & $\mathrm{M}$ & Q & Q & Q & $\mathrm{Q}$ & Q & $\mathrm{S}$ & $\mathrm{S}$ & $\mathrm{S}$ & $\mathrm{S}$ & $M$ \\
\hline All American Canal & $\mathrm{C}$ & $\mathrm{C}$ & $\mathrm{M}$ & $\mathrm{M}$ & $\mathrm{C}$ & $\mathrm{M}$ & - & $\mathrm{M}$ & $\mathrm{M}$ & $\mathrm{M}$ & $\mathrm{M}$ & $\mathrm{M}$ & - & $\mathrm{M}$ & - & $\mathrm{M}$ & - & - & - & - & - & - & - & - & - & $\mathrm{M}$ \\
\hline \multicolumn{27}{|c|}{ Salton Sea - Surface Water and Sediment } \\
\hline SS-1 (North Basin) (at depths) & $\mathrm{C}$ & $\mathrm{C}$ & $\mathrm{M}$ & $\mathrm{M}$ & $\mathrm{C}$ & $\mathrm{M}$ & $\mathrm{M}$ & Q & Q & Q & Q & Q & $\mathrm{M}$ & $\mathrm{M}$ & Q & Q & Q & A & A & A & A & - & A & $\mathrm{S}$ & A & $\mathrm{M}$ \\
\hline SS-2 (mid-Sea) (at depths) & $\mathrm{C}$ & $\mathrm{C}$ & $\mathrm{M}$ & $\mathrm{M}$ & $\mathrm{C}$ & $\mathrm{M}$ & $\mathrm{M}$ & Q & Q & Q & Q & Q & $\mathrm{M}$ & $\mathrm{M}$ & Q & Q & Q & A & A & A & A & - & A & $\mathrm{S}$ & A & $\mathrm{M}$ \\
\hline SS-3 (South Basin) (at depths) & $\mathrm{C}$ & $\mathrm{C}$ & $\mathrm{M}$ & $\mathrm{M}$ & $\mathrm{C}$ & $\mathrm{M}$ & $\mathrm{M}$ & Q & Q & Q & Q & Q & $\mathrm{M}$ & $\mathrm{M}$ & Q & Q & Q & $\mathrm{A}$ & $\mathrm{A}$ & $\mathrm{A}$ & $\mathrm{A}$ & - & $\mathrm{A}$ & $\mathrm{S}$ & $\mathrm{A}$ & $\mathrm{M}$ \\
\hline New River Delta locations & $\mathrm{M}$ & $\mathrm{C}$ & M & $\mathrm{M}$ & $\mathrm{C}$ & $\mathrm{M}$ & $\mathrm{M}$ & Q & Q & Q & Q & Q & $\mathrm{M}$ & Q & Q & Q & Q & A & A & A & A & & A & $\mathrm{S}$ & A & $\mathrm{M}$ \\
\hline Alamo River Delta locations & $\mathrm{M}$ & $\mathrm{C}$ & $\mathrm{M}$ & $\mathrm{M}$ & $\mathrm{C}$ & $\mathrm{M}$ & $\mathrm{M}$ & Q & Q & Q & Q & Q & $\mathrm{M}$ & Q & Q & Q & $\mathrm{Q}$ & $\mathrm{A}$ & $\mathrm{A}$ & $\mathrm{A}$ & $\mathrm{A}$ & - & $\mathrm{A}$ & $\mathrm{S}$ & $\mathrm{A}$ & $\mathrm{M}$ \\
\hline
\end{tabular}

KEY: - = not measured, $\mathrm{C}=$ continuous, $\mathrm{M}=$ monthly, $\mathrm{Q}=$ quarterly, $\mathrm{S}=$ seasonal, based on seasons of use, $\mathrm{A}=$ annual

*Fish and invertebrate samples will be collected as part of the biological resources element of the monitoring plan. 
Table 14. Salton Sea geography and geology monitoring questions and information needs.

Question
How are drainage patterns being changed in the watershed?
How much area will be exposed as the Salton Sea shoreline
recedes in the future?
What are sources of potential airborne dusts?
Where do the potential airborne dusts originate?
How does erosion and deposition affect shoreline and delta
areas? What is the rate of erosion and sedimentation
(deposition)?

What type of land uses around the lake could affect or benefit restoration activities?

How much development will occur around the lake?

Where is the distribution of housing and infrastructure?

What are changes in agricultural uses?

How does faulting change topography (for example, fault scarps and offsets)?

What is the distribution of vegetation?

What is the distribution of soils?

How will geothermal development affect the landscape?

Where are locations of geologic hazards? What are the earthquake hazards and recurrence intervals?

Where does subsidence occur and at what rate?

What are geographical changes that may occur as a result of climate change? What are the effects of climate change on inflows?

Where are the locations of snags and islands?

What is the distribution of benthic communities on the seafloor?

What areas are prone to landslides and slumps?
Topography and contours

Land use

Land cover

National Hydrogaphy Dataset (NHD) base

Bathymetric elevations and contours

Salton Sea Inflows

Salton Sea Outflows

Properties, distribution, and thickness of finegrained sediment in the lake

Remote sensing data

Topography

Sedimentation rate and transport

Silt loads in rivers

Sediment cores

Land use

Land use

Land cover

Transportation

Orthoimagery

Land use

Land cover

Surface water (canals and ditches)

Orthoimagery

Topography

Land cover

Color infrared orthoimagery

Land cover

Color infrared orthoimagery

County soil survey

Geothermal activity and locations of hotspots Land use

Faulting rupture (displacements)

Seismic monitoring

Locations of geothermal vents

Volcanic eruptions

Areas prone to landslides and slumps

Liquefaction potential areas

Subsidence (derived from elevation)

Topography

Water-surface elevations

Bathymetry

Survey of snags and islands

Bathymetry and side-scan data

Topographic maps, orthoimagery, geologic reports, and engineering reports. 
Table 15. Salton Sea air-quality monitoring questions and information needs. Question

Information Needs

What are the existing conditions and trends for air quality at the Salton Sea?

Ambient concentrations of pollutants of concern:

Particulate matter $\left(\mathrm{PM}_{\text {coarse }}\left(\mathrm{PM}_{10-2.5}\right)\right.$ and $\left.\mathrm{PM}_{2.5}\right)$

Ozone and NOx

Sulfur dioxide, hydrogen sulfide, and ammonia

Analysis/speciation of collected PM filter samples ${ }^{\text {a }}$

Particulate matter deposition rates ${ }^{1}$

What are the surface meteorological conditions at the Salton Sea?

Wind speed and wind direction

Temperature

Solar radiation

Precipitation ${ }^{\text {a }}$

Evaporation $^{\mathrm{a}}$

Relative humidity

Barometric pressure

${ }^{1}$ Data that may be collected as part of monitoring and/or future focused investigations. 
Table 16. Objectives and purposes of air-quality monitoring activities.

Objective

Measure ambient concentrations of the pollutants of concern at and near the Salton Sea.

Monitor changes in ambient concentrations of pollutants of concern at the Salton Sea over time.

\section{Purpose}

Document current air pollutant concentrations

Document seasonal changes in concentrations

Establish benchmarks against which data gathered during

long-term monitoring can be compared

Support potential future environmental documentation associated with restoration

Document variability and trends in air pollutant concentrations at and near the Salton Sea

Provide basis for clarifying factors and relationships that influence air pollutant concentrations at and near the

Salton Sea (for example, contributing emission sources; pollutants of concern; meteorological parameters; playa, groundwater, and sand conditions)

Provide basis for projecting potential air pollutant concentrations under different restoration scenarios Provide information to support design and management of ecosystem restoration elements, such as created habitats

Support potential environmental documentation associated with restoration 
Table 17. Salton Sea regional monitoring stations.

\begin{tabular}{|c|c|c|c|c|c|c|c|c|}
\hline Name & Address & PM10 & $\begin{array}{l}\text { Continuous } \\
\text { PM10 }\end{array}$ & PM2.5 & $\begin{array}{l}\text { Continuous } \\
\text { PM2.5 }\end{array}$ & 03 & NO2 & $\begin{array}{l}\text { Meteorological } \\
\text { Parameters }\end{array}$ \\
\hline $\begin{array}{l}\text { Torres Martinez } \\
\text { Tribal Lands }^{1}\end{array}$ & Tribal Headquarters & $x$ & & & & $x$ & & $x$ \\
\hline $\begin{array}{l}\text { Whitewater River } \\
\text { Outlet }\end{array}$ & $\begin{array}{l}\text { Torres Martinez Tribal } \\
\text { Lands }^{1}\end{array}$ & $x$ & $x$ & $x$ & $x$ & & & $x$ \\
\hline Palm Springs & $\begin{array}{l}590 \text { E Racquet Club } \\
\text { Av, Palm Springs } \\
92262\end{array}$ & $x$ & & $x$ & & $x$ & $x$ & $x$ \\
\hline Indio & $\begin{array}{l}46990 \text { Jackson St, } \\
\text { Indio } 92201\end{array}$ & $x$ & $x$ & $x$ & & $x$ & & $x$ \\
\hline Niland & $\begin{array}{l}7711 \text { English Rd., } \\
\text { Niland } 92257\end{array}$ & $x$ & $x$ & & & $x$ & & $x$ \\
\hline Westmorland & $\begin{array}{l}570 \text { Cook St., } \\
\text { Westmorland } 92281\end{array}$ & $x$ & & & & $x$ & & $x$ \\
\hline Brawley & $\begin{array}{l}220 \text { Main St., } \\
\text { Brawley } 92227\end{array}$ & $x$ & $x$ & $x$ & & & & $x$ \\
\hline El Centro & $\begin{array}{l}150 \text { 9th St, } \\
\text { El Centro } 92243\end{array}$ & $x$ & & $x$ & & $x$ & $x$ & $x$ \\
\hline $\begin{array}{l}\text { Calexico - } \\
\text { East }\end{array}$ & $\begin{array}{l}1699 \text { Carr Rd, } \\
\text { Calexico } 92231\end{array}$ & & & & $x$ & $x$ & $x$ & $x$ \\
\hline $\begin{array}{l}\text { Calexico - } \\
\text { Ethel St. }\end{array}$ & $\begin{array}{l}1029 \text { Belcher St, } \\
\text { Calexico } 92231\end{array}$ & $x$ & & $x$ & $x$ & $x$ & $x$ & $x$ \\
\hline $\begin{array}{l}\text { Calexico - } \\
\text { Grant St. }\end{array}$ & $\begin{array}{l}900 \text { Grant St, } \\
\text { Calexico } 92231\end{array}$ & $x$ & & & & $x$ & & $x$ \\
\hline Bombay Beach & $\begin{array}{l}\text { A St. \& 3rd St., } \\
\text { Bombay Beach } 92257\end{array}$ & $x$ & $x$ & $x$ & $x$ & & & $x$ \\
\hline $\begin{array}{l}\text { Salton Sea Naval } \\
\text { Test Site }\end{array}$ & $\begin{array}{l}\text { State Highway } 86, \\
\text { approximately } 16.5 \\
\text { miles northwest of } \\
\text { Westmorland, } \\
\text { California }\end{array}$ & $x$ & $x$ & $x$ & $x$ & & & $x$ \\
\hline $\begin{array}{l}\text { Salton Sea South } \\
\text { Shore }\end{array}$ & $\begin{array}{l}906 \text { W Sinclair Rd., } \\
\text { Calipatria } 92233\end{array}$ & $x$ & $x$ & $x$ & $x$ & & & $x$ \\
\hline $\begin{array}{l}\text { Salton Sea } \\
\text { Recreation Area }\end{array}$ & $\begin{array}{l}\text { 100-225 State Park } \\
\text { Rd., North Shore } \\
92254\end{array}$ & $x$ & $x$ & $x$ & $x$ & & & $x$ \\
\hline Salton City & $\begin{array}{l}\text { Salton Community } \\
\text { Services District } \\
\text { wastewater treatment } \\
\text { plant }\end{array}$ & $x$ & $x$ & $x$ & $x$ & & & $x$ \\
\hline
\end{tabular}

${ }^{1}$ Source: http://www.torresmartinez.org/Departments/EPA/Air/tabid/69/Default.aspx

${ }^{2}$ Note: The Calexico-Grant station was decommissioned in 2007. 
Table 18. On-going monitoring tracks.

\begin{tabular}{lcccc}
\hline Type of Study & Initial Stage & $\begin{array}{c}\text { Intermediate } \\
\text { Stages }\end{array}$ & $\begin{array}{c}\text { Concluding } \\
\text { Stage }\end{array}$ & Comments \\
\hline Focus Groups & $\mathrm{X}$ & $\mathrm{X}$ & $\mathrm{X}$ & \\
Survey & $\mathrm{X}$ & $\mathrm{X}$ & $\mathrm{X}$ & \\
\hline
\end{tabular}




\section{Appendices}




\section{Appendix 1. Protocol for Monitoring Birds in Agricultural Areas (from ERA, 2007)}

Associated Species (Spring): White-faced ibis (spring and winter), white-tailed kite (spring and winter), Swainson's hawk (spring--migrant only), American peregrine falcon (spring and winter), western snowy plover (spring and winter), long-billed curlew (spring and winter), black tern (spring migrant only), loggerhead shrike (spring and winter), and tricolored blackbird (spring and winter).

Associated Species (Winter): Aleutian Canada goose, fulvous whistling duck, greater sandhill crane, white-faced ibis (spring and winter), bald eagle, white-tailed kite (spring and winter), ferruginous hawk, American peregrine falcon (spring and winter), merlin, prairie falcon, western snowy plover (spring and winter), mountain plover, long-billed curlew (spring and winter), loggerhead shrike (spring and winter), and tricolored blackbird (spring and winter).

Specific Habitat Parameters: Vegetative cover, crop height, type of crop or current status (fallow, plowed, burned), and presence of standing water or mud.

Survey Period: March to May (spring) and November to February (winter).

Protocol: In order to increase the area surveyed and thereby increasing the probability of detecting agricultural bird species that utilize large, and often "ephemeral" foraging areas a transect survey protocol with a 4-kilometer long linear transect (fig. 4) will be utilized to census agricultural bird species. Each transect is associated with a circular panel, to the extent possible. There are 11 points within each transect, which are 400 meters apart. A team of two biologists will stop at each point for up to five (5) minutes. Surveying for agricultural species will occur within 300 feet of the center line of each transect. All covered species, number of individuals, habitat, and behavioral code will be recorded within each transect, including at the scanning points.

To ensure covered bird species will not be affected by the team's presence, the distance between each point will be driven no greater than five (5) miles per hour. Approximately half of the panels will be sampled in this manner. In addition to binoculars, a spotting scope will be utilized by each team of surveyors to confirm identification. Agricultural fields will be mapped during each survey, as these fields change regularly throughout the year. The crop category, crop stage, crop condition, crop type, and crop height will be recorded.

The two spring surveys are proposed, one in late March and April and another in mid to late May. The two winter surveys will be performed during the November/December and January/February months. As presented in Table 11, all survey panels with a composition of greater than 30 percent agricultural fields (approximately 26 sentinel panels and 121 rotating panels [approximately 40 panels per year]) will be surveyed for agricultural bird species. These surveys will be performed with the Raptor Bird Surveys when feasible. 


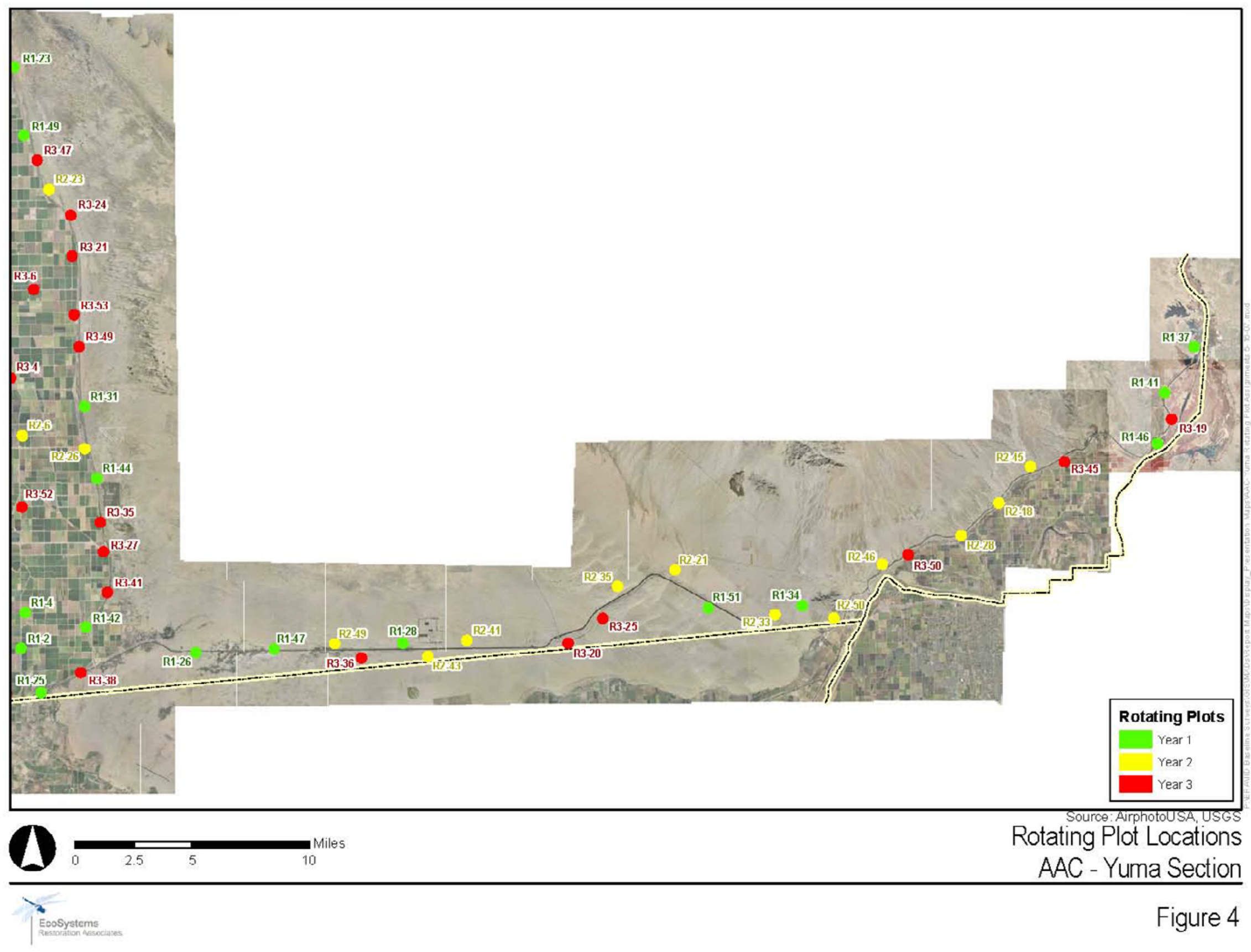


Table 11

Number of Panels Grouped into Three Classes of Agriculture Based on Percent Cover

\begin{tabular}{|c|c|c|c|c|}
\cline { 2 - 5 } \multicolumn{1}{c|}{} & $\begin{array}{c}\text { AG CLASS }=3 \\
(>70 \% \mathrm{Ag})\end{array}$ & $\begin{array}{c}\text { AG CLASS }=2 \\
(30-70 \% \mathrm{Ag})\end{array}$ & $\begin{array}{c}\text { AG CLASS }=1 \\
(<30 \% \mathrm{Ag})\end{array}$ & Total \\
\hline Sentinel & 17 & 9 & 17 & 43 \\
\hline Rotating & 106 & 15 & 38 & 159 \\
\hline Total & 123 & 24 & 55 & 202 \\
\hline
\end{tabular}




\title{
Appendix 2. Key Questions Identified by the Hydrology and Water Quality Focused Technical Group
}

\author{
Hydrology (Inflows/Outflows/Storage)
}

How refined are our understandings of the system plumbing and water budgets?

What are the current quantity, quality, and trends of inflows to the Salton Sea?

a. What are the relative contributions of various sources of inflow? (New River, Alamo River, Direct Drains, Whitewater River, San Felipe Creek, Salt Creek, groundwater, precipitation)

What are the appropriate levels of precision and accuracy for quantity, quality, and trend data?

What are the spatial and temporal patterns in the quality and quantity of inflows to the Salton Sea?

What current and future activities in the watershed, including imports and exports, could affect the quality and quantity of inflows to the Salton Sea?

a. Mexico policy decisions (for example, phosphate, inflow availability)

b. Cropping patterns

c. Tile/Tailwater contributions

d. Colorado River management

e. Urbanization

f. Water quality regulations (for example, TMDL)

What are the effects of inflows on lake elevations and associated exposure of playa?

a. How do we reconcile differences between the USGS and IID elevations datums? What elevation datum will be the program standard? (coordinate with Geophysical workgroup)

What data are necessary to characterize climatological trends and hydrologic processes?

a. What meteorological and climatological data are necessary in and around the lake? (wind speed, evaporation, temperature) (coordinate with Air Quality workgroup)

What is the extent of the study watershed for groundwater (for example, elevation) and surface water?

What is the relationship of the hydrologic budget to the salinity of the Salton Sea?

Water Quality and Sediment Quality

What are the key water and sediment quality parameters necessary to characterize baseline and future habitat quality and function at the Salton Sea? (link to Biology and Air Quality workgroups)

a. Salinity, selenium (speciation), temperature, DO, turbidity, $\mathrm{pH}$ nutrients, ammonia, ReDox, $\mathrm{H} 2 \mathrm{~S}$, sulfur (speciation), total organic carbon, grain size, chlorophyll, dynamics (stratification/ destratification), pesticides, arsenic

b. What are the current and future trends of sediment loading, quality, and deposition?

i. How are adsorbed constituents affected when sediment reaches the Salton Sea?

What are the key water quality and sediment parameters necessary to assess baseline and future ecological risk at the Salton Sea?

a. See previously 
What are the key water quality and sediment data necessary to characterize eutrophic conditions of the Salton Sea?
a. Nutrient inflow
b. temperature, DO, turbidity, $\mathrm{pH}$, nutrients, ammonia, ReDox, H2S, sulfur (speciation), total organic carbon, grain size, chlorophyll, dynamics (stratification/ de-stratification), Secchi depth
c. aerial/satellite observations (episodic, algal and gypsum blooms, fish die-offs)

How are important water quality parameters cycled in the Salton Sea?
a. Carbon cycle
b. Selenium cycle
c. Sulfur cycle
d. Nutrient cycle $(\mathrm{P}, \mathrm{N})$ (fish as a P sink)
e. Circulation, suspension, stratification/destratification, decomposition, sinks, volatilization,

Does salinity stratification occur?

a. What is the importance of salinity stratification in selenium cycling?

b. What is the extent of the freshwater lens, if any? Duration?

c. Would salinity stratification affect mixing zones in constructed habitats?

What are the critical changes over time within the watershed affecting the quality of inflows?

What processes (for example, deposition) are important to characterize the transport of water and water quality parameters?

Will the lake develop carbon dioxide sinks that could be released during lake turnover, posing a human health risk?

\section{Groundwater}

What is the relationship between groundwater movement and the elevation of the Salton Sea, including potential locations of constructed habitats?
a. What is the extent of the basin of influence affecting/affected by the Salton Sea?
b. What is the transmissivity of the aquifer?
c. What is the estimated hydraulic conductivity of the lakebed?

What are the physical and hydraulic conditions within the groundwater system(s)? (for example, hydrostratigraphy, physical, chemical, head conditions, water quality, geologic structure)

How is Sea water quality affected by groundwater levels and the elevation of the Salton Sea?

What are the trends in groundwater conditions affecting the Salton Sea over time?

How can the interaction of groundwater with the lake, atmospheric conditions, and land surface be characterized?

a. As the lake recedes, when and where will seeps develop? If they do, will they mitigate some air quality concerns and/or will there be potential to develop them as a water supply for constructed habitats?
b. What is the relationship between groundwater levels and formation of salts at the surface of exposed playa?


What is the potential risk that dewatering will contribute to subsidence?

What are the groundwater sources, sinks, and changes in storage?

What are the localized effects of other constructed wetlands (for example, Imperial Valley wetlands, Torres Martinez wetland, other proposed projects) on groundwater?

How do the geothermal zones influence groundwater conditions?

What are the groundwater quality parameters of interest?

a. Perchlorate?

b. Trace elements?

Hydrodynamics

What creates the stratification//destratification dynamics?

a. Thermal stratification

b. Circulation

c. Mixing

d. Pycnocline (spatial extent, duration, biological productivity)

What wave height and interval data are necessary, spatially and temporally? (wind wave re-suspension, circulation) 
Appendix 3. Conceptual Models for Selenium, Nutrient, and Sulfur Cycling at the Salton Sea

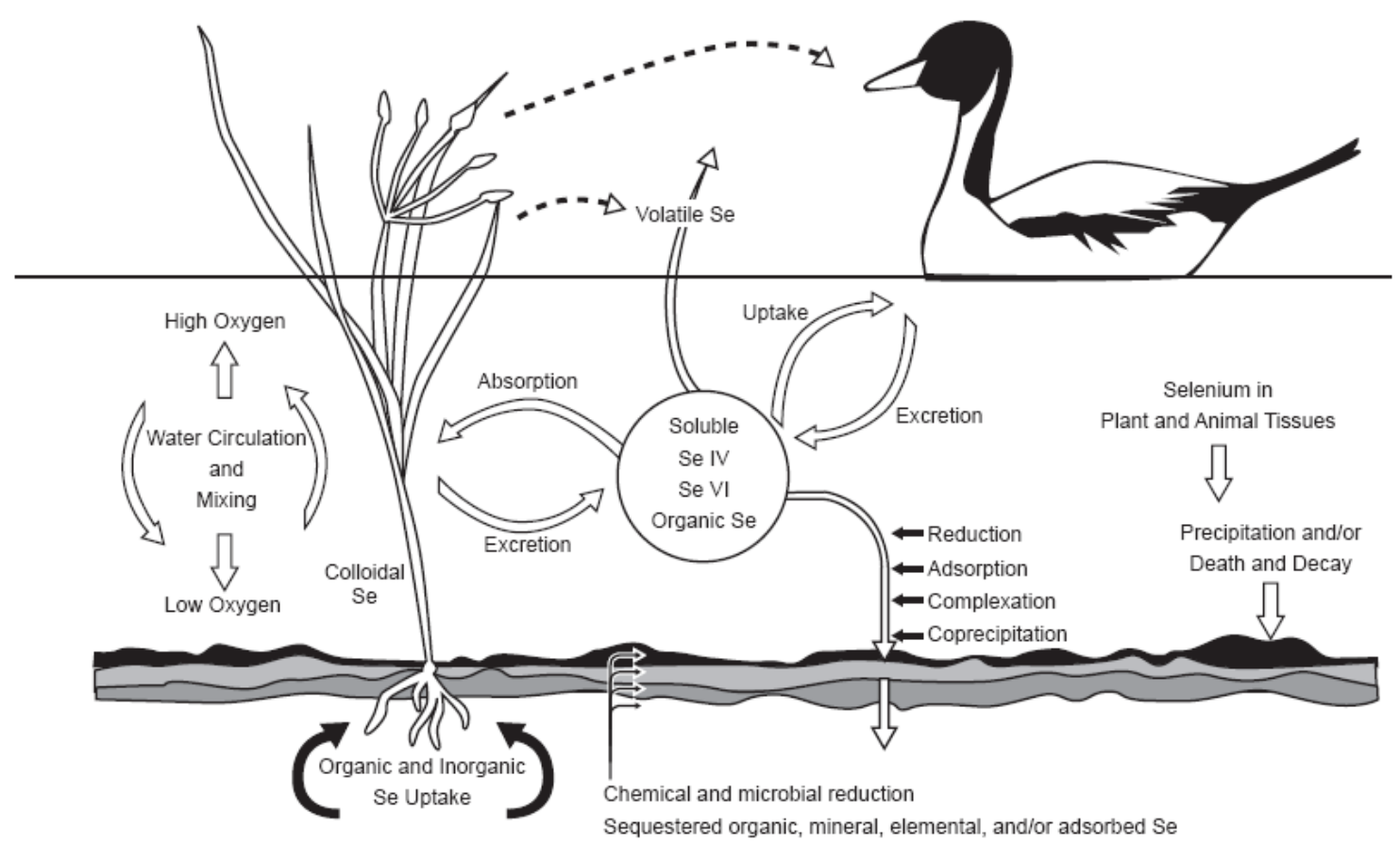

Figure A4-1. Selenium Cycling in an Aquatic Ecosystem

Source: Lemly \& Smith, 1987 

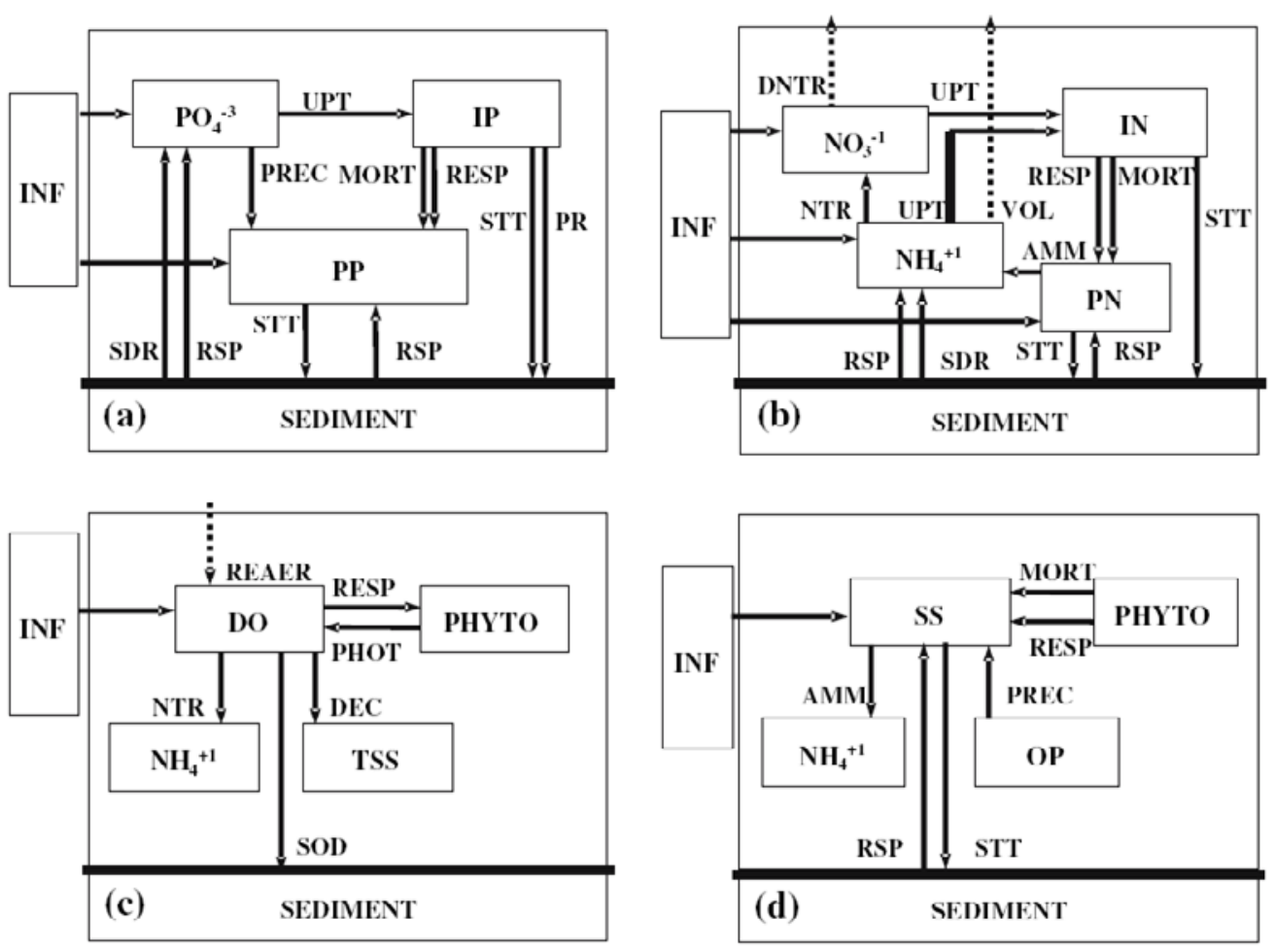

Figure A4-2. Conceptual models for the Salton Sea: (a) phosphorus; (b) nitrogen; (c) DO; (d) suspended solids.

Source: Chung and others, 2008

Solid lines indicate process in the water column and between water and sediment. Dashed lines indicate processes between air and water surface. INF = Inflow; UPT = Uptake; RESP = Respiration; MORT = Mortality; PR = Permanent Removal; SDR = Sediment Release; $\mathrm{STT}=$ Settling; RSP = Sediment Resuspension; PREC $=$ Precipitation + adsorption; NTR $=$ Nitrogen; DNTR $=$ Denitrification; $\mathrm{VOL}=$ Volatilization; $\mathrm{AMM}=$ Ammonification; REAER $=$ Reaeration; SOD $=$ Sediment Oxygen Demand; PHOT $=$ Photosynthesis; DEC $=$ Decomposition. 


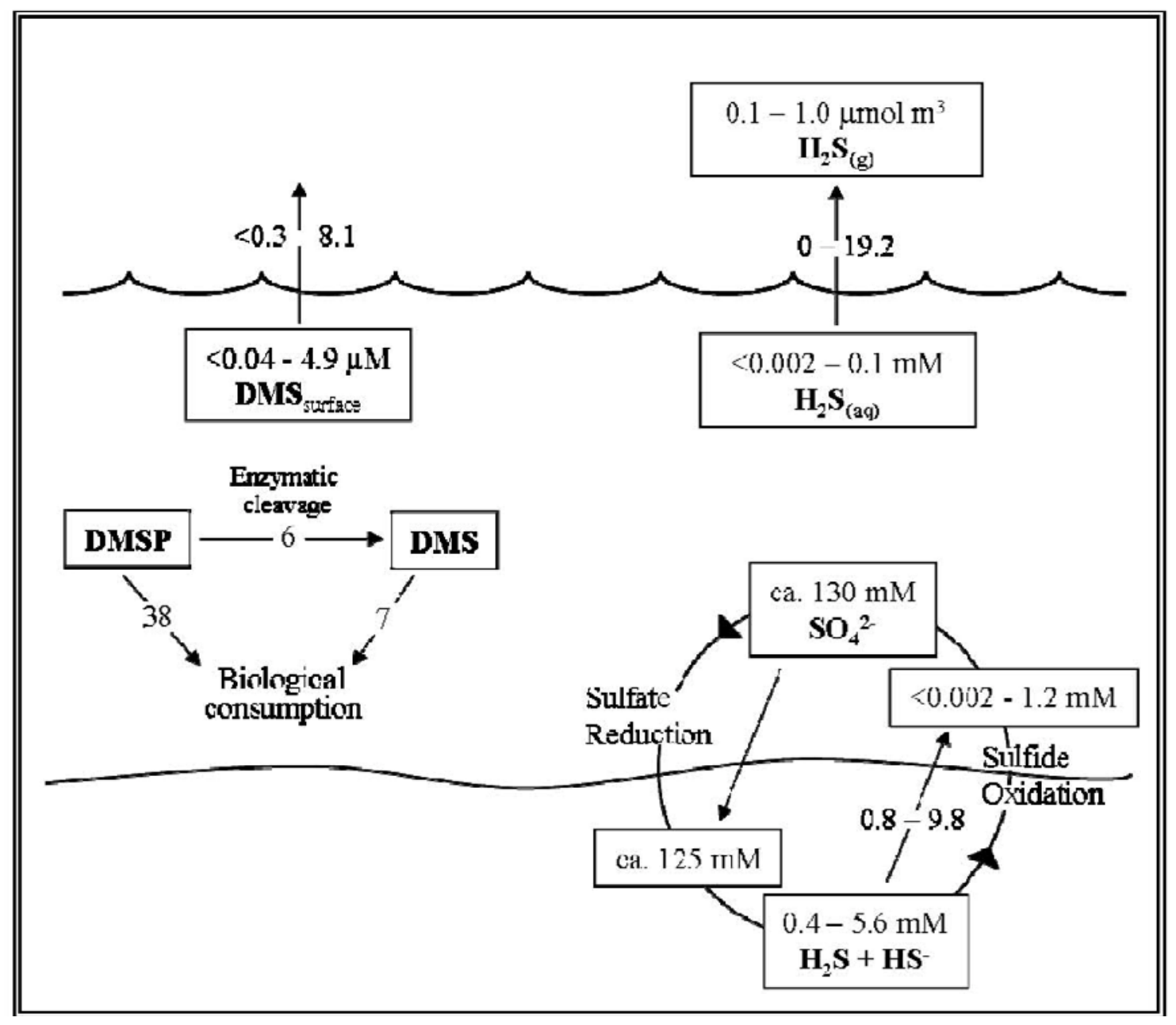

Figure A4-3. Graphical depiction of the sulfur biogeochemical cycle at the Salton Sea.

Source: Reese, 2007

Measured concentrations are provided in boxes. Estimated fluxes are unboxed and given as mmol m-2 d-1 
Appendix 4. SWAMP Quality Assurance Project Plan Template

Draft Report

\title{
Salton Sea Monitoring and Assessment: Quality Assurance Project Plan
}

\author{
Project Name
}

Prepared for

California Department of Water Resources

May 2008

Prepared by

Revision No. 0 


\section{Approval Signatures}

Title

Name

Signature

Date ${ }^{1}$

Project Manager

QA Officer

\section{Notes}

1. This is a contractual document. The signature dates indicate the earliest date when the project can start. 



\section{Table of Contents}

Approval Signatures .............................................................................................................................. i

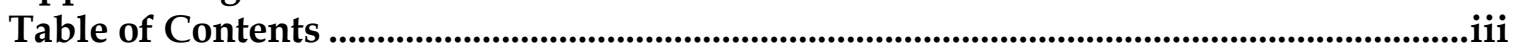

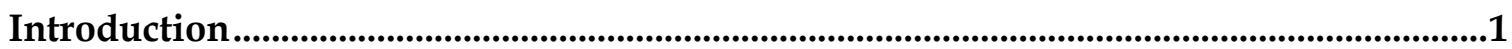

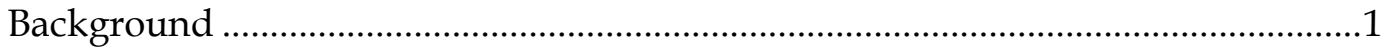

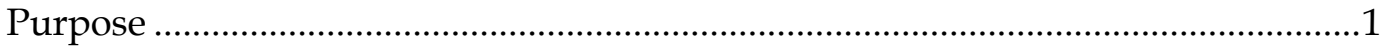

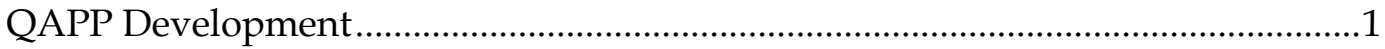

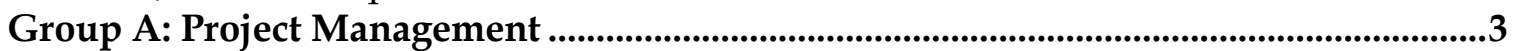

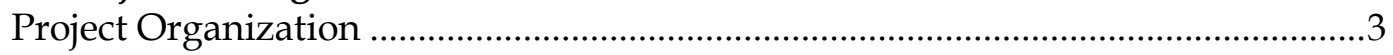

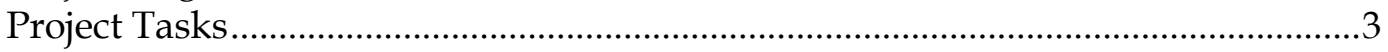

Data Quality Objectives and Criteria......................................................................

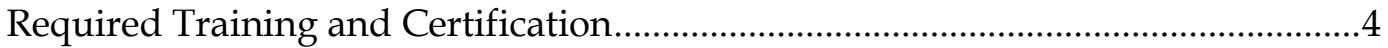

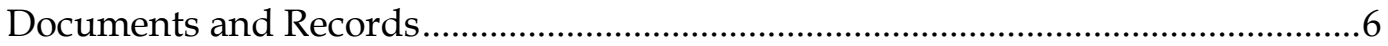

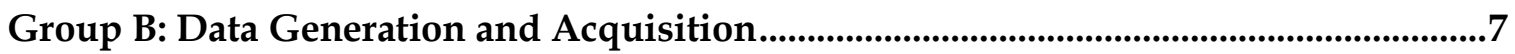

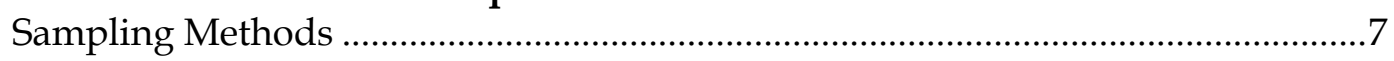

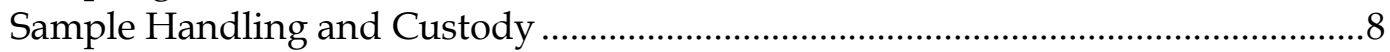

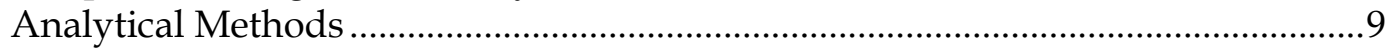

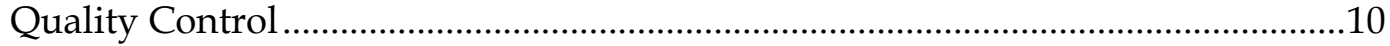

Instrument/Equipment Testing, Inspection, and Maintenance ..............................12

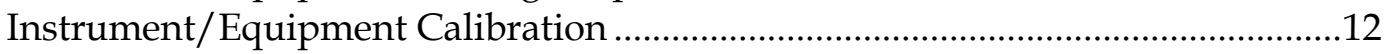

Inspection/ Acceptance of Supplies and Consumables ........................................13

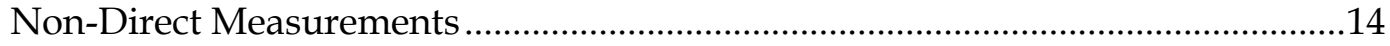

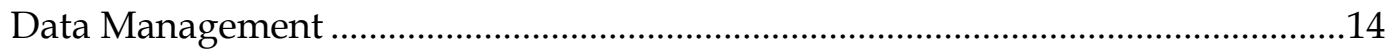

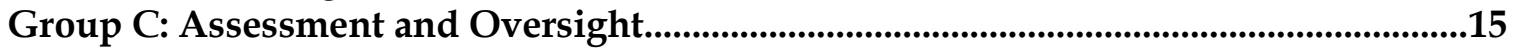

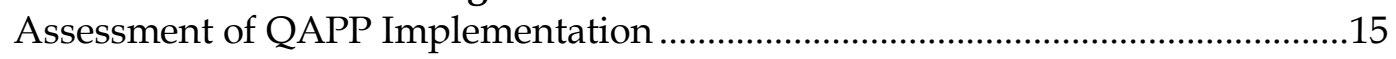

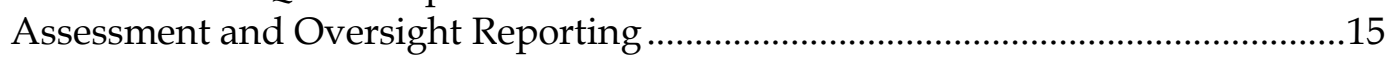

Group D: Data Validation and Usability .......................................................................................15

Data Verification and Validation Criteria ..............................................................17

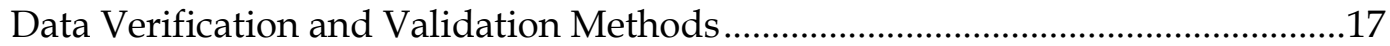

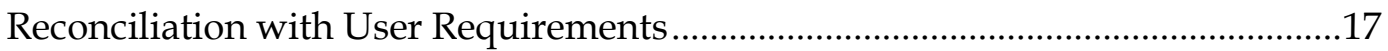




\section{Introduction}

\section{Background}

The California Departments of Water Resources (DWR) and Fish and Game (CDFW) have identified the need to develop a Monitoring and Assessment Program that will begin to collect information that will support future decisions regarding actions to restore the Salton Sea ecosystem and to help guide the design and management of restoration features and habitats that might be created. It includes a description of long-term monitoring and assessment, beginning in Fiscal Year 2008-2009.

\section{Purpose}

The purpose of this report is to provide a quality assurance project plan (QAPP) for the water and sediment quality monitoring described in the Hydrologic Monitoring and Assessment Plan.

\section{QAPP Development}

The development of this report was based in part on use of the US Environmental Protection Agency's (USEPA) Guidance for Quality Assurance Project Plans (USEPA, 2002) and the California State Water Resources Control Board (SWRCB) Surface water Ambient Monitoring Program (SWAMP) Electronic Template for SWAMP Compatible Quality Assurance Project Plans (SWRCB, 2004). Both of these documents provide a logical stepwise process for the development of an effective QAPP. 


\section{Distribution List}

Title

Name / Affiliation

Project Manager

QA Officer

\begin{tabular}{l}
\hline \\
\hline Project Manager \\
\hline QA Officer \\
\hline
\end{tabular}

Telephone No. No. of Copies

n




\section{Group A: Project Management}

\section{Project Organization}

\section{Involved Parties and Roles}

- Identify lead agencies and any other partner agencies

- List of individuals and organizations involved with the project, identifying their roles and responsibilities; include both information producers and information users as well as contractors

TABLE

Personnel Responsibilities and QA Project Plan Receipt

\begin{tabular}{|c|c|c|c|c|}
\hline Name & Organization & Title & $\begin{array}{c}\text { Contact } \\
\text { Information }\end{array}$ & $\begin{array}{c}\text { QAPP } \\
\text { Receipt/Control \# }\end{array}$ \\
\hline
\end{tabular}

Notes:

1. Telephone number, fax number, email address

\section{Quality Assurance Officer}

- Documentation of Project quality assurance (QA) manager's independence from those generating project information

\section{QAPP Updates and Maintenance}

- Identification of individual responsible for maintaining the official, approved QAPP

- Identify who can make changes to the QAPP

\section{Organization and Responsibilities}

- Organizational chart showing relationships and lines of communication among project personnel

- Chart will show names, titles, and relationships and lines of communication among project personnel 


\section{Project Tasks}

\section{Description of Work}

- Summary of work to be performed and products

- Indicate constituents to be monitored and the measurement processes and techniques to be used

\section{Schedule}

- Describe the Project schedule

TABLE

Task Schedule

Activity

Initiation Date

Completion Date

Deliverable

Deliverable Due Date

\section{Location}

- Maps, tables, and so on showing geographic locations

\section{Constraints}

Describe resource and/or time constraints, if applicable

\section{Data Quality Objectives and Criteria}

- Provide quality specifications

- Provide established measurement performance or acceptance criteria

- Express criteria in terms of precision, accuracy, bias, representativeness, comparability, completeness, and sensitivity 
TABLE

Data Quality Objectives for Field Measurements

Group Parameter Accuracy Precision Recovery Target Reporting Completeness
Limit

TABLE

Data Quality Objectives for Laboratory Measurements

\begin{tabular}{ccccc}
\hline Group Parameter Accuracy Precision & Recovery & $\begin{array}{c}\text { Target Reporting } \\
\text { Limit }\end{array}$ & Completeness \\
\hline
\end{tabular}

\section{Required Training and Certification}

\section{Specialized Training and Certification Requirements}

- List and describe any specialized training and certifications needed by personnel, if any.

\section{Documentation}

- Describe plans for providing, documenting, and assuring this training and certification is obtained

- Identify where documentation will be located

\section{Training and Certification Providers}

- Provide information on who will be providing the training 
TABLE

Special Personnel Training and/or Certification

Training

Training Provider

Completion Date

Trainee/Organization

Proof of Training

\section{Documents and Records}

- List of records to be included in the data report package

- List of any other project documents to be produced

- Provide information on final disposition of records and documents, including location and retention schedule

- Identify persons responsible for maintaining records.

- Describe of how the most current approved QAPP will be distributed to project staff

- Discuss back-up plans for record stored electronically

TABLE

Document and Record Retention, Archival, and Disposition Information

\begin{tabular}{ccccc}
\hline Record Type & $\begin{array}{c}\text { Required } \\
\text { Documentation }\end{array}$ & Retention & Archival & Disposition \\
\hline
\end{tabular}

Sample Collection

Field

Analytical

Data

Assessment

Modeling 


\section{Group B: Data Generation and Acquisition}

\section{Sampling Design}

- Reference MAP

- Type and total number of samples needed

- Where samples will be taken

- What to do if the site becomes inaccessible

- Project activity schedules

- Identify sources of variability and how variability will be reconciled with Project information

\section{Sampling Methods}

- Description of sample/data collection procedures

- List of equipment needed

- Identification of performance requirements

- Description of corrective actions to be taken if problems arise

TABLE

Sampling Locations and Methods

\begin{tabular}{|c|c|c|c|c|c|c|c|c|c|c|}
\hline 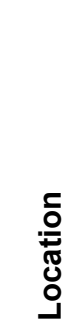 & 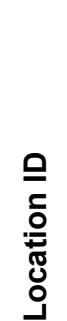 & 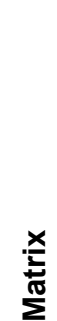 & 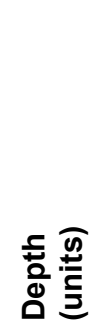 & 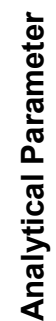 & 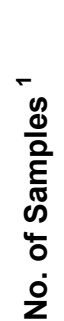 & 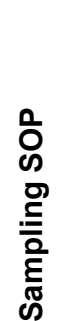 & 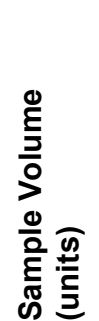 & 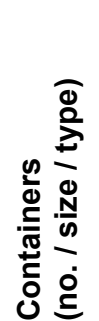 & 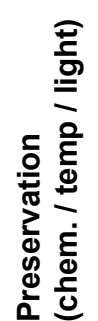 & 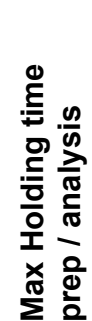 \\
\hline
\end{tabular}

Notes

1. Include field duplicates 


\section{Sample Handling and Custody}

- Description of how samples will be handled, transported, and then received in the laboratory or office

- Provide sample handling and custody procedures requirements and transfer, and for ultimate disposal

TABLE

Sample Handling and Custody Responsibility

\begin{tabular}{l}
\hline \multicolumn{1}{c}{ Sample Handling Activity } \\
\hline Sample Collection, Packaging and Shipment \\
Sample Collection \\
Sample Packing \\
Coordination of Shipment \\
Type of Shipment (Courier) \\
\hline Sample Receipt and Analysis \\
Responsible Organization or Individual \\
Sample Receipt \\
Sample Custody and Storage \\
Sample Preparation \\
Sample Determinative Analysis \\
\hline Sample Archival \\
Field Sample Storage (\# days from sample \\
collection) \\
Sample Extract/Digestate Storage (\# days from \\
extraction/digestion): \\
\hline Sample Disposal \\
Responsible Organization \\
Responsible Personnel
\end{tabular}


TABLE

Sample Handling and Custody

\begin{tabular}{lllll}
\hline Parameter & Container & Volume & Initial Preservation & Holding Time \\
\hline
\end{tabular}

\section{Analytical Methods}

- Description of analytical methods to be used

- Identification of any performance criteria

- Description of corrective actions when problems arise

TABLE

Field Analytical Methods

\begin{tabular}{|c|c|c|c|c|c|c|c|}
\hline \multirow[t]{2}{*}{ Analyte } & \multirow[t]{2}{*}{$\begin{array}{l}\text { Laboratoryl } \\
\text { Organization }\end{array}$} & \multirow{2}{*}{$\begin{array}{l}\text { Project } \\
\text { Action } \\
\text { Limit }\end{array}$} & \multirow{2}{*}{$\begin{array}{c}\text { Project } \\
\text { Quantification } \\
\text { Limit }\end{array}$} & \multicolumn{2}{|c|}{ Analytical Method } & \multicolumn{2}{|c|}{$\begin{array}{c}\text { Achievable Lab } \\
\text { Limits }\end{array}$} \\
\hline & & & & $\begin{array}{l}\text { Analytical } \\
\text { Method/ } \\
\text { SOP }\end{array}$ & $\begin{array}{l}\text { Modified } \\
\text { yes/no }\end{array}$ & MDLs & Method \\
\hline
\end{tabular}




\section{Quality Control}

- List of QC activities needed for sampling, analytical, or measurement techniques, along with their frequency

- Description of control limits for each QC activity and corrective actions when these are exceeded

- Identification of any applicable statistics to be used

TABLE

Project Quality Control Checks

\begin{tabular}{|c|c|}
\hline QC Check & Information Provided \\
\hline \multicolumn{2}{|l|}{ Blanks } \\
\hline bottle blank & cleanliness of sample bottles \\
\hline field blank & transport, storage, and field handling bias \\
\hline reagent blank & contaminated reagent \\
\hline rinsate or equipment blank & contaminated equipment \\
\hline method blank & response of an entire laboratory analytical system \\
\hline \multicolumn{2}{|l|}{ Spikes } \\
\hline matrix spike & analytical (preparation + analysis) bias \\
\hline matrix spike replicate & analytical bias and precision \\
\hline analysis matrix spike & instrument bias \\
\hline surrogate spike & analytical bias \\
\hline \multicolumn{2}{|l|}{ Calibration Check Samples } \\
\hline zero check & calibration drift and memory effect \\
\hline span check & calibration drift and memory effect \\
\hline mid-range check & calibration drift and memory effect \\
\hline \multicolumn{2}{|l|}{ Replications, Splits, And so on } \\
\hline field collocated samples & sampling + measurement precision \\
\hline field replicates & precision of all steps after acquisition \\
\hline field splits & shipping + interlaboratory precision \\
\hline laboratory splits & interlaboratory precision \\
\hline laboratory replicates & analytical precision \\
\hline analysis replicates & instrument precision \\
\hline
\end{tabular}


TABLE

Field Sampling Quality Control

\section{Matrix}

Sampling SOP

Analytical Parameter(s)

Analytical Method/SOP Reference

No. Sample Locations

\section{Field QC}

Equipment Blanks

Field Blanks

Trip Blanks

Cooler Temperature

Field Duplicate Pairs

Collocated Samples

Field Splits

Field Matrix Spikes

Others

TABLE

Analytical Quality Control

\section{Matrix}

Sampling SOP

Analytical Parameter(s)

Analytical Method/SOP Reference

No. Sample Locations

\section{Field QC}

Method Blanks

Reagent Blanks

Storage Blanks

Instrument Blank

Lab Duplicate

Lab Matrix Spike

Matrix Spike Duplicate

Lab Control Sample 


\section{Instrument/Equipment Testing, Inspection, and Maintenance}

- List of equipment and/or systems needing periodic maintenance, testing, or inspection; provide a schedule for these activities

- Description of how inspections and periodic preventive maintenance procedures will be performed and documented

- Discussion on how critical spare parts will be supplied and stocked

- Description of how re-inspections will be performed and effectiveness of corrective actions

TABLE

Testing, Inspection and Maintenance of Sampling Equipment and Analytical Instruments

\begin{tabular}{|c|c|c|c|c|c|c|c|c|}
\hline 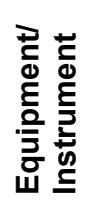 & 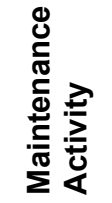 & 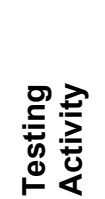 & 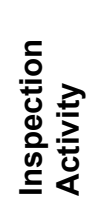 & 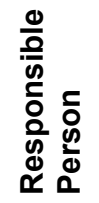 & 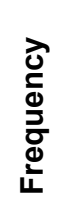 & 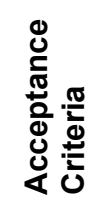 & 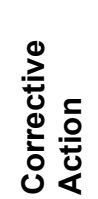 & 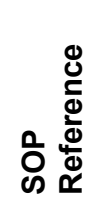 \\
\hline
\end{tabular}

\section{Instrument/Equipment Calibration}

- List of all project tools, gauges, instruments, and other sampling, measuring, and test equipment that will be calibrated

- Description of calibration method and identification of any certified equipment and/or standards to be used 
- Details of how calibration records will be maintained and traceable to the instrument/equipment

TABLE

Equipment and Instrumentation Calibration

\begin{tabular}{lllcccc}
\hline $\begin{array}{l}\text { Equipment/ } \\
\text { Instrument }\end{array}$ & Procedure & $\begin{array}{c}\text { Calibration } \\
\text { Frequency }\end{array}$ & $\begin{array}{c}\text { Acceptance } \\
\text { Criteria }\end{array}$ & $\begin{array}{c}\text { Corrective } \\
\text { Action }\end{array}$ & $\begin{array}{c}\text { Organization/Person } \\
\text { Responsible }\end{array}$ & $\begin{array}{c}\text { SOP } \\
\text { Reference }\end{array}$ \\
\hline
\end{tabular}

\section{Inspection/Acceptance of Supplies and Consumables}

- A list of project supplies and consumables that may directly or indirectly affect the quality of the results

- The acceptance criteria

- Identification of those responsible

TABLE

Inspection/Acceptance Testing Requirements for Consumables and Supplies

$\begin{array}{ccccc}\begin{array}{c}\text { Project-Related } \\ \text { Supplies/Consumables }\end{array} & \begin{array}{c}\text { Inspection/Testing } \\ \text { Specifications }\end{array} & \begin{array}{c}\text { Acceptance } \\ \text { Criteria }\end{array} & \text { Frequency } & \begin{array}{c}\text { Responsible } \\ \text { Individual }\end{array}\end{array}$




\section{Non-Direct Measurements}

- Identification of any existing data that will be obtained from non-measurement sources, such as literature files and historical databases

- Description of how this data will be used

- Acceptance criteria and any limitations for using such data

\section{Data Management}

- Description of the project data management process

- Description of or reference to the office's standard record-keeping procedures and document control, data storage, retrieval, and security systems

- Identification of data handling equipment and procedures to process, compile, and analyze project data

- Discussion of data handling procedures to detect and correct errors and loss during data processing

- Examples of any forms or checklists to be used

- Identification of any specific computer hardware/software performance requirements and how configuration acceptability will be determined

- Description of how applicable information resource management requirements will be satisfied, as well as any applicable Agency information resource management requirements 


\section{Group C: Assessment and Oversight}

\section{Assessment of QAPP Implementation}

- Description of project assessments planned and a brief discussion of the information expected

- Approximate schedule for these assessments and their reports

- For any planned self assessments, identification of potential participants and their relationship within the project organization

- For independent assessments, identification of the organization and person(s) that will perform the assessments

- Identification of how, when, and to whom the results of each assessment will be reported

- Description of the corrective action process; include how actions are to be addressed, by whom, and how they are verified and documented

TABLE

Assessment

Type

Frequency

Internal or External

Person, Title, Organizational Affiliation Responsible for:

Assessment Response Corrective Monitoring
Actions

Effectiveness

\section{Assessment and Oversight Reporting}

- Frequency and distribution of reports to inform management of the project's status

- Identification of report preparer and recipients, as well as any specific actions or recommendations recipients are expected to make 
TABLE

Management Reports

Record Type

Frequency

Projected Delivery

Responsible

Report Recipients Person/Organization 


\section{Group D: Data Validation and Usability}

\section{Data Review, Verification, and Validation Criteria}

- State the criteria for deciding to accept, reject, or qualify project data in an objective and consistent manner

- If applicable, include any subsequent activities and criteria that will review data a second time for data that fails to pass the first review

\section{Data Verification and Validation Methods}

- Description of how project data will be verified and validated

- Discussion of how any issues will be resolved and identification of who has the authority for resolving them

- Description of how results will be conveyed to data users

- Explanation of how validation issues differ from verification issues for this project

- Examples of any forms or checklists to be used and identification of any project specific calculations

TABLE

Data Validation Summary

Matrix

Parameter

Concentration

Validation

Validation Criteria

Data Validatorl

Level

Criteria

Modification

Organization

\section{Reconciliation with User Requirements}

- Description of how project results will be reconciled with the requirements defined by the data user or decision maker 
- An outline of methods proposed to analyze the data and determine possible anomalies or departures from assumptions made when the project was planned

- Description of how reconciliation with user requirements will be documented, issues will be resolved, and how limitations on the use of the data will be reported to decision makers 


\section{Appendix 5. Legacy and Current-Use Pesticides Detected in the Salton Sea}

\begin{tabular}{|c|c|c|c|c|c|c|c|c|c|c|c|}
\hline \multirow[b]{2}{*}{ Citation } & \multirow[b]{2}{*}{ Constituent } & \multicolumn{2}{|c|}{$\begin{array}{c}\text { High Threshold } \\
\text { Sediment Concentration }\end{array}$} & \multicolumn{3}{|c|}{$\begin{array}{l}\text { Bed Sediment Concentrations } \\
(\mathrm{ng} / \mathrm{g} \mathrm{dw}) \text { - May } 2000^{3}\end{array}$} & \multicolumn{3}{|c|}{$\begin{array}{l}\text { Bed Sediment Concentrations } \\
\text { (ng/g dw) - May } 2001\end{array}$} & \multicolumn{2}{|c|}{$\begin{array}{c}\text { Concentrations in Corvina } \\
\text { (ng/g wet wt) collected in the } \\
\text { Salton Sea, May } 2001^{6}\end{array}$} \\
\hline & & $\begin{array}{l}\text { Probable } \\
\text { Effect } \\
\text { Level } \\
\text { (PEL) })^{1}\end{array}$ & $\begin{array}{l}\text { Effect } \\
\text { Range- } \\
\text { Median } \\
(\text { ERM) })^{2}\end{array}$ & $\begin{array}{l}\text { Salton Sea- } \\
\text { Middle }\end{array}$ & $\begin{array}{l}\text { Salton Sea- } \\
\text { South\#1 }\end{array}$ & $\begin{array}{l}\text { Salton Sea- } \\
\text { North \#1 }\end{array}$ & $\begin{array}{l}\text { Salton Sea- } \\
\text { Middle }\end{array}$ & $\begin{array}{l}\text { Salton Sea - } \\
\text { South \#3 }\end{array}$ & $\begin{array}{l}\text { Salton Sea - } \\
\text { North \#2 }\end{array}$ & Muscle & Liver \\
\hline \multirow{18}{*}{$\begin{array}{l}\text { Sapozhnikova and } \\
\text { others (2004) }\end{array}$} & Trifluralin & & & $<0.16$ & 3.2 & 4 & 2.6 & 1.2 & 1.8 & 0.9 & 5.1 \\
\hline & Dimethoate & & & 1.3 & 2.3 & 2.2 & $<0.2$ & $<0.2$ & $<0.2$ & 0.2 & 1.2 \\
\hline & Diazinon & & & 1.4 & 0.5 & 2 & 5.4 & 4.3 & 0.6 & 5.4 & 17.2 \\
\hline & Malathion & & & $<0.15$ & 5.2 & 7.2 & $<0.15$ & $<1.15$ & $<0.15$ & 0.1 & 1 \\
\hline & Endrin & & & $<0.13$ & $<0.13$ & $<0.13$ & $<0.13$ & $<0.13$ & $<0.13$ & 0.3 & 1.2 \\
\hline & Lindane & 1.38 & & 1.4 & $<0.13$ & 3.6 & 0.7 & 1.8 & 0.7 & 6.7 & 19.7 \\
\hline & Chlorothalonil & & & $<0.12$ & 6.6 & 8.9 & 2.3 & 1.5 & 2.9 & 2.2 & 10.3 \\
\hline & Dachtal & & & 1.2 & 6 & 2.6 & $<0.13$ & $<0.13$ & $<0.13$ & 9.1 & 12.9 \\
\hline & Dursban & & & 4.1 & 9.5 & $<0.12$ & $<0.12$ & $<0.12$ & $<0.12$ & 0.6 & 2.1 \\
\hline & Disulfoton & & & $<0.20$ & $<0.20$ & $<0.20$ & 29.6 & $<0.20$ & $<0.20$ & $<0.14$ & 0.2 \\
\hline & $\mathrm{HCB}$ & & & $<0.2$ & $<0.2$ & $<0.2$ & $<0.2$ & 0.7 & $<0.2$ & 1.5 & 18.6 \\
\hline & Haptachlor & & & 0.3 & 4.5 & 1.1 & 1.3 & $<0.17$ & 0.9 & 2 & 26.6 \\
\hline & Dieldrin & 6.67 & & 12.3 & 3 & $<0.2$ & 1.4 & 0.5 & 3.9 & 2 & 10.2 \\
\hline & Total DDE & 8.51 & & 30.6 & 25.3 & -- & 18.9 & 27.2 & 18.8 & 8.7 & 76.1 \\
\hline & Total DDD & & & 6.7 & -- & -- & 3.3 & -- & 2.6 & 4.7 & 17.7 \\
\hline & Total DDT & & 46.1 & 1.9 & -- & -- & 1.5 & -- & 1.6 & 4.3 & 35 \\
\hline & Sum DDT4 & & & 40.2 & 25.3 & -- & 23.7 & 27.2 & 23 & 17.6 & 128.8 \\
\hline & Total PCBs ${ }^{5}$ & 277 & 180 & 180.9 & 283.6 & 304 & 179.3 & 116.1 & 299.3 & 30.21 & 78.51 \\
\hline
\end{tabular}

${ }^{1}$ MacDonald and others (2000).

${ }^{2}$ Long and others (1995).

${ }^{3}$ Values in bold exceed the sediment threshold.

${ }^{4}$ Sum DDT $=$ Total DDT+Total DDE+Total DDD.

${ }^{5}$ Total PCBs measured in sediment $=$ sum of 42 congeners.

${ }^{6}$ Values represent the mean of $\mathrm{N}=6$ samples. 


\section{Appendix 6. Legacy and Current-Use Pesticides Detected in Tributaries}

\begin{tabular}{|c|c|c|c|c|c|c|c|c|c|c|}
\hline \multirow[b]{2}{*}{ Citation } & \multirow[b]{2}{*}{ Constituent } & \multicolumn{4}{|c|}{ Aqueous Concentrations (ng/L) Spring 2002} & \multicolumn{4}{|c|}{ Suspended Sediment Concentrations (ng/g) Spring 2002} & \multirow{2}{*}{$\begin{array}{l}\text { Human Oral } \\
\text { Dose Low } \\
\text { Effects Level } \\
\quad(\mathrm{mg} / \mathrm{kg})\end{array}$} \\
\hline & & $\begin{array}{l}\text { Alamo } \\
\text { River } \\
\text { Outlet }\end{array}$ & $\begin{array}{l}\text { New } \\
\text { River } \\
\text { Outlet }\end{array}$ & $\begin{array}{c}\text { Whitewater } \\
\text { River } \\
\text { Outlet }\end{array}$ & $\begin{array}{l}\text { Aqueous } \\
\mathrm{MDL}\end{array}$ & $\begin{array}{l}\text { Alamo } \\
\text { River } \\
\text { Outlet }\end{array}$ & $\begin{array}{l}\text { New } \\
\text { River } \\
\text { Outlet }\end{array}$ & $\begin{array}{c}\text { Whitewater } \\
\text { River } \\
\text { Outlet }\end{array}$ & $\begin{array}{l}\text { Sediment } \\
\text { MDL } \\
\text { (ng/L) }\end{array}$ & \\
\hline \multirow{14}{*}{$\begin{array}{l}\text { LeBlanc, and others } \\
\text { (2004) }\end{array}$} & Atrazine & 630 & 434 & $(2.1)$ & 4.2 & & & & 0.6 & \\
\hline & Carbofuran & 530 & nd & nd & 3.3 & nd & nd & 5.1 & 3 & \\
\hline & Chlorpyrifos & 73.1 & 20.7 & nd & 4.2 & & & & 1.5 & 0.1 \\
\hline & Dacthal & 47 & 31.7 & 6.9 & 1.2 & 7.1 & nd & 2.2 & 0.6 & 10 \\
\hline & Diazinon & (3.4) & 22.7 & nd & 3.6 & nd & nd & 2 & 1.5 & 214 \\
\hline & EPTC (eptam) & 50.6 & nd & nd & 4.5 & (3.7) & 5.6 & nd & 0.7 & \\
\hline & Malathion & 1100 & 253 & nd & 2.1 & & & & 1.5 & \\
\hline & Oxyflurofen & 30.3 & nd & nd & 4.2 & 19.7 & nd & nd & 6.1 & \\
\hline & Pendimethalin & 156 & 65.3 & nd & 2.4 & 42.4 & nd & nd & 4 & \\
\hline & Trifluralin & 600 & 215 & nd & 3 & 106.4 & 33.3 & (.6) & 1.4 & 150 \\
\hline & Methidathion & & & & & nd & 34.3 & nd & 3.4 & \\
\hline & $p, p^{\prime}-$ DDD & & & & & 4.9 & nd & nd & 2.9 & \\
\hline & $p, p^{\prime}-\mathrm{DDE}$ & & & & & 101 & 58.2 & 31.7 & 3.7 & $0.9-1.5$ \\
\hline & p,p'-DDT & & & & & nd & nd & nd & 3.9 & \\
\hline $\begin{array}{l}()=\text { Concentration } \\
\text { below MDL }\end{array}$ & & & & & & & & & & \\
\hline
\end{tabular}




\title{
Appendix 7. Key Questions Identified by the Air Quality FTG
}

\author{
Monitoring to Characterize Baseline or Current Ambient Air Quality Conditions
}

What are the existing conditions and trends of air quality at the Salton Sea?

What ambient concentrations of PM10, PM2.5, ozone, NOx, SOx, hydrogen sulfide, and ammonia (and any other identified pollutants) represent baseline or current conditions at the Salton Sea?

What is the quality and adequacy of the data from existing air-quality monitoring locations in the Salton Sea Air Basin?

What additional data will be needed for future project level effect evaluations?

Are new monitoring stations needed to expand the existing monitoring network to measure baseline and future levels of the pollutants of concern at the Salton Sea?

Are there other pollutants, including potential hazardous air pollutants, which should also be considered? How can data on these pollutants be collected by using the existing and/or expanded air-quality monitoring network at the Salton Sea?

What is the best way to enhance/expand the existing monitoring stations to better collect needed data on baseline concentrations of pollutants at the Salton Sea?

\section{Monitoring to Characterize Baseline or Current Surface Meteorological Conditions}

What are the existing surface meteorological conditions and trends at the Salton Sea (wind speed, wind direction, temperature, relative humidity, barometric pressure, solar radiation, precipitation, evaporation, and so on)?

What is the quality and adequacy of the data from existing meteorological monitoring locations in the Salton Sea Air Basin?

What additional data will be needed for future project level effect evaluations?

What correlations can be developed for data on meteorological conditions, such as wind speed, when measurements are taken concurrently at the same location at different heights previously the ground? What information on local surface roughness conditions can be developed from wind speed measurements taken concurrently at the same location at different heights previously the ground?

Can/should the 2 meter CIMIS data be used in future studies?

Chemical Analysis of Collected Particulate Matter (Dust) Samples

What is the chemical composition of the particulate matter in ambient air sampled along the shoreline under baseline or current conditions? In particular, are there measureable concentrations of hazardous or toxic air pollutants in the sampled windblown dust?

Is the chemical composition of the particulate matter in ambient air currently sampled along the Salton

Sea shoreline, including potential hazardous air pollutants, representative of that expected for future conditions?

\section{Particle Deposition Study}

What are typical particle deposition rates for windblown dust from exposed playa under baseline or current conditions? Are these deposition rates representative of that expected for future conditions?

What is the chemical composition of the deposited particulate matter? 
Will windblown dust result in particle deposition of sufficient mass or chemical composition to harm area agriculture?

Playa Characterization: Dust Emission Potential, Salt Crust Genesis, Chemical Composition, Physical Characteristics

How much playa will be exposed, over what time frame, and where will the exposed playa be located?

For exposed playa available for near term studies, what are the threshold wind velocities and associated particulate matter emission rates, for both disturbed and undisturbed playa? How can the threshold wind velocities and associated particulate matter emissions for existing disturbed and undisturbed playa be characterized by using ambient measurements collected and averaged in hourly and subhourly averaging periods? Are the threshold wind velocities and associated particulate matter emission rates identified for exposed playa in near term studies representative of future conditions for disturbed and undisturbed playa?

For exposed playa available for near term studies, how does dust emission potential vary in relation to time exposed and distance from the shoreline?

For exposed playa available for near term studies, how does salt crust genesis and change vary in relation to time exposed and distance from the shoreline?

For exposed playa available for near term studies, how does the chemical composition of playa soils and salts vary in relation to time exposed and distance from the shoreline?

For exposed playa available for near term studies, how do physical characteristics of playa soils and salts vary in relation to time exposed and distance from the shoreline?

Is the chemical composition of the playa currently sampled along the Salton Sea shoreline, including potential hazardous substances, representative of that expected for windblown dust or particulate matter in ambient air under future conditions?

For exposed playa available for near term studies, are there physical and/or chemical surface conditions that can be correlated with dust emission potential? Can these surface conditions be mapped and used for predictions of potential playa emissions and/or needs for control measure implementation?

\section{Groundwater Interactions}

How does groundwater presence or absence affect the exposed playa: dust emission potential, salt crust genesis, chemical composition, and physical characteristics? To what extent does the depth to groundwater contribute to, or inhibit, particulate matter emission rates from exposed playa?

How does groundwater presence or absence influence the effectiveness of dust control measures (for example, water efficient vegetation, salt crust, etc.)?

\section{Sand Surveys}

What sources of sand exist at the Salton Sea with potential to contribute to fugitive dust emissions, what are their characteristics, and where are they located? To what extent is sand present on existing and potential future exposed areas?

For exposed playa available for near term studies, what sand motion is occurring and to what extent is moving sand contributing to dust emissions from exposed areas? Are the sand motion characteristics and dust generation rates identified for exposed playa in near term studies representative of future conditions? 


\section{Upper Air Meteorological Monitoring}

What are the ranges of atmospheric mixing heights for the Salton Sea Air Basin?

How do atmospheric mixing heights vary diurnally and seasonally near the Salton Sea?

\section{Microclimate Conditions}

What microclimatic conditions represent baseline or current conditions adjacent to the Salton Sea?

What equipment is needed to monitor microclimatic conditions adjacent to the Salton Sea? What parameters should be monitored? How long and at what frequency should microclimatic conditions be monitored? Where should monitors be located?

How might microclimatic conditions be expected to change in the future?

Remote Sensing and Satellite Imagery

How might remote sensing and satellite imagery be used to track changes in exposed Salton Sea shoreline areas?

\section{Back Trajectory Analysis}

How might back trajectory analysis be used to predict the sources of monitored particulate matter at the Salton Sea?

\section{Mitigation and Control Measures}

What methods represent best available control measures (BACM) for:

a. Construction equipment exhaust emissions (NOx, PM10),

b. Construction fugitive dust emissions (PM10), and

c. Playa fugitive dust emissions (PM10)?

What is the effectiveness of the methods in the "Tool Box of Control Measures" for control of playa fugitive dust emissions (PM10)? What pilot and demonstration studies are needed to demonstrate effectiveness of the measures at the Salton Sea?

How does the IID Water Conservation and Transfer Project Mitigation Monitoring and Reporting Plan (MMRP) Four-Step Plan interact with studies or projects that may be undertaken as part of the ecosystem restoration program?

\section{Odor Studies}

What odorous emissions and sources currently affect air quality at the Salton Sea? Are these emissions and sources representative of future conditions?

Modeling of Impacts

What tools are needed and what models should be used to predict future air quality conditions in the Salton Sea Air Basin and at the Salton Sea?

Greenhouse Gas (GHG) Emissions and Climate Change Impacts

How might climate change affect evaporation rates and soil surface structure in the Salton Sea Air Basin? 
How might climate change affect wind speed, direction, and frequency in the Salton Sea Air Basin?

How will air quality and meteorology in the Salton Sea Air Basin be affected by global climate change?

Will ecosystem restoration projects emit greenhouse gases (GHGs) at levels of significance? If so, how will GHG emissions be mitigated?

\section{Data Management}

How should data be stored and who should have access to the data?

What platform should be used for storage and access?

How will the monitoring data be analyzed?

Who will do the data analysis and interpretation, and how will the results of these analyses and interpretations be made available? 


\section{Appendix 8. CDFW Metadata Standards}

Please provide these items for each dataset.

Abstract: Briefly describe what the dataset is about (who, what, where, when). Include any limitations of the dataset, assumptions made, and if there is anything special that the user of these data should be aware of.

Purpose: Briefly describe why the dataset was created.

Date: The date or range of dates when the data were gathered, or the date the photos, maps or other items at the core of the dataset, were created.

Point of Contact: Contact information for an individual or organization that is knowledgeable about the dataset. Include:

Person's Name: Complete first and last name

Organization's Name: Program, administrative unit, and agency, company, or group name

Telephone Number: Including Area Code

E-Mail address:

Field Definitions: List and define each field used in your shapefile, database, or spreadsheet.

Abbreviation Definitions: For any field that contains numeric or alphabetic

codes (for example, $\mathrm{SAC}=$ Sacramento County), list each code/abbreviation and provide an unabbreviated definition.

Scale: Is your dataset intended to be viewed/used at a particular scale? If so what?

Access Constraints: Is there a need to limit who has access to see or read this dataset? If so, specify. If not, put "None."

Use Constraints: Is there a need to limit the use of this dataset to certain people or to specific tasks? If so, specify, If not, put "None." Also include how the data should be cited, if you want something specific.

Data Distribution: Can your data be distributed? If yes, to who? If no, can you be contacted for distribution?

Progress: Complete or Incomplete.

Update Frequency: Possible values are: Continually, Daily, Weekly, Monthly, Annually, Unknown, As Needed, Irregular, None Planned, or ..... 
If you are providing a GIS file (shapefile, etc.), these next two items are often defined as part of that file, but you will need to make sure.

Projection: What is the Projected Coordinate System name?

California Teale Albers (preferred)

Latitude/Longitude

State Plane

UTM

Datum (or Geographic Coordinate System): Which Datum is the projection in?

NAD83

NAD27

(GCS_North_American_1983) (preferred)

WGS84

(GCS_North_American_1927)

(WGS_1984)

Keywords (optional): Words or short phrases summarizing an aspect of the dataset, used to allow people to find your dataset with quick keyword searches.

Theme Subjects covered by the dataset.

Place Geographic locations characterized by the dataset.

Metadata must be provided in XML format. XML formatted data can be created by using ESRI software. If ESRI software is not available standalone, metadata editors can be found at http://www.fgdc.gov/metadata/geospatial-metadata-tools\#availabletools 


\section{Appendix 9. Standards That Enable You to Contribute Your Data to Larger Data Sets}

One of the most important places you can contribute your data are to the growing number of databases that are developing at national, regional, and global levels around the world. If you contribute your data, then other practitioners can make use of your findings and learn from your experiences. In order to make your data accessible to outside parties, they need to conform to international data standards that provide the basis for open sharing of data. These standards need to occur on several levels:

Standard Software Formats - Your data need to be in an electronic format that other users can either directly read or at least import. For example, most people can read files generated by Microsoft programs that use the *.doc, ${ }^{*}$.xls and *.mdb formats for documents, spreadsheets and databases respectively. Non-proprietary formats include HyperText Markup Language (HTML) used for webpages or, in more recent years, various flavors of eXtensible Mark-up Language (XML). For example, in the world of spatial data, ESRI's Geography Markup Language (GML) is becoming the standard XML encoding for geospatial information.

Standard Data - Your data need to fit the structure of the databases to which you contribute. As a simple example, if you are reporting numbers of birds of different species in a census and your data are in the form of nesting pairs whereas the database wants to know individual adult birds, your data will not be compatible unless they are converted. Most databases will outline the format that data need to be in.

Standard Terms - A particularly important aspect of the need for standard data is the need for standard terminology. If you have recorded plant names by using locally common names, these data will useable more broadly. For biological names, it is important to use scientific (Latin) names; the Integrated Taxonimic Information System (ITIS, http://www.itis.gov/) is a standard reference. Similarly, if you call a threat "cattle grazing" and another project terms it "livestock" then there will be no way to compare results.

Specific resources that you may wish to consult for terminology include:

- Geographic Place Names - GeoNet Names Server (GNS): http://gnswww.nga.mil/geonames/GNS/index.jsp

- Biological Species and Other Taxonomic Information - Integrated Taxonomic Information System (ITIS): $h t t p: / / w w w . i t i s . g o v /$

- Habitats, Threats, and Conservation Actions - IUCN/CMP Classifications \& Authority Files: http://www.redlist.org/info/authority_files

- General Terms: California Environmental Resources Evaluation System (CERES): http://gis.ca.gov/catalog/thesaurus.epl?mode=browse or General Multilingual Environmental Thesaurus (GEMET): http://www.eionet.europa.eu/gemet

Standard Metadata - In addition to contributing your data themselves, you should also contribute meta information about your dataset. Typical metadata include identification of the data being described, the source of the data and a contact person/organization, the quality of the data, entity, or attribute information (if in a database or spreadsheet), its publication date, distribution information (including rights/liabilities), and the name of the individual completing the metadata. Metadata are typically recorded in a separate file (often by using HTML or XML) that accompanies the main data 
file. The US Federal Geographic Data Committee has created a metadata standard that is fairly widely accepted throughout the world and is suggested as a good metadata reference to employ. For more information on this standard, please go to: http://www.fgdc.gov/metadata/geospatial-metadatastandards. 


\title{
Appendix 10. Memorandum of Understanding (DRAFT) This is a template subject to revision and additions.
}

\section{MEMORANDUM OF UNDERSTANDING}

\author{
Among the \\ California Department of Fish and Game \\ California Department of Water Resources \\ California Air Resources Board \\ California State Water Resources Control Board \\ regarding collaboration and development of Data Management for the Salton Sea
}

\section{Section 1. Preamble and Background}

During the past few years, State and Federal agencies have collected data on the fish, wildlife, air and water resources at the Salton Sea. These data are the foundation for analyses used to make decisions affecting Salton Sea management and restoration. The recent advent of tools to link databases through server systems and high-speed data transfer technology, and the capability by many to display and serve these data to a broad audience by using Internet Map Services (IMS), have led to centralized solutions for data storage, management, and retrieval.

The Salton Sea Data Management Program is intended as a statewide program to meet multi-agency information storage and retrieval needs. The purpose of the Salton Sea Data Management Program is to provide centralized Salton Sea data to partner agencies and to the public; and to function as a multi-agency cooperative program designed to gather, maintain, and disseminate Salton Sea fish, wildlife, air, water, socioeconomic, and geologic resource data and data standards. As such, the system is intended to accommodate a wide variety of data and information including life history and species accounts, population trends, habitat data, barrier data, distribution information, bathymetric data, imagery, and so on. The stated goal is as follows:

To create, maintain, and enhance high quality, consistent data that are directly applicable to policy, planning, management, research, and restoration of the Salton Sea; and

To provide data and information services in a timely manner in formats that meet the needs of users.

Currently, data and information are offered through the Biogeographic Information and Observation System (BIOS) and the Document Library web sites. BIOS is a system designed to enable the management, visualization, and analysis of biogeographic data collected by the Department of Fish and Game (CDFW) and its Partner Organizations. In addition, BIOS facilitates the sharing of those data within the BIOS community. BIOS integrates GIS, relational database management, and ESRI's ArcIMS technology to create a statewide, integrated information management tool that can be used on any computer with access to the Internet. The Document Library is a tool for managing important documents held by the CDFW. The Library stores and makes readily available key documents frequently used by the public, Department employees and our cooperators.

As additional information and data are identified and evaluated for suitability for BIOS and the Document Library, they will be added to the growing content and made available to agencies cooperating with the CDFW and the public.

\section{Section 2. Purpose}

The intent of this Memorandum of Understanding (MOU) among the California Department of Fish and Game, California Department of Water Resources (DWR), California Air Resources Board (ARB), and California State Water Resources Control Board (SWRCB) (hereinafter "Cooperators") is to outline initial roles and responsibilities for development and use of the Salton Sea data and information management, stewardship, and dissemination program. 


\section{Section 3. Authorities and Obligations}

a. Nothing herein grants any party to this MOU powers and authorities that they do not otherwise possess under the constitutions, laws, and rules of the State of California or of the United States.

b. Nothing herein limits or affects in any way the controlling authorities or obligations of the parties to this MOU.

c. It is recognized by all parties that participation in the MOU does not guarantee or assure the issuance of any approvals or permits required under the applicable statutory authorities. It is further recognized that the decision to issue said approvals or permits remains within the sole discretion of the appropriate resource agency.

\section{Section 4. Roles and Responsibilities}

Roles and responsibilities of specific Cooperators follow:

a. CDFW agrees to maintain a Data Management plan, define contract deliverables and budget expectations for Data Management; establish and promulgate standards for data documentation; establish data transfer and storage protocols; provide a digital data repository to make data easily accessible to partners and the public and in a useable format; provide GIS and GPS support for biological surveys and analysis; provide necessary hardware and software to meet the Salton Sea Data Management objectives; and fund staff services as required.

b. DWR agrees to deliver high quality data in an acceptable and useable digital format for end users including summarizations of raw data; provide data updates as they become available; ensure all deliverable products contain metadata that meets the Federal Geographic Data Committee (FGDC) and BIOS standards; ensure all deliverables be submitted during the funding period; provide derived data products such as reports, summaries, graphs, and so on to the CDFW; and include the following verbiage in all contracts entered into by DWR:
All data collected and created for this contract is a required deliverable of this contract and will become the property of the State of California, and not of the contracted party. A condition of final payment on this contract shall include the delivery of all related data. Spatial data should be delivered in an ESRI useable format where applicable and documented with metadata in accordance with minimum BIOS metadata standards (http://bios.dfg.ca.gov/metadata.asp) and FGDC metadata standards (http://www.fgdc.gov/metadata/documents/workbook_0501_bmk.pdf).

DWR further agrees to participate as a member of the Salton Sea Program, providing Salton Sea related expertise in water related issues such as water quality, monitoring, evaluating and sighting of proposed infrastructure.

c. ARB agrees to deliver high quality data in an acceptable and useable digital format for end users including summarizations of raw data; provide data updates as they become available; ensure all deliverable products contain metadata that meets the Federal Geographic Data Committee (FGDC) and BIOS standards; ensure all deliverables be submitted during the funding period; provide derived data products such as reports, summaries, graphs, and so on to the CDFW; and include the following verbiage in all contracts entered into by ARB:

All data collected and created for this contract is a required deliverable of this contract and will become the property of the State of California, and not of the contracted party. A condition of final payment on this contract shall include the delivery of all related data. Spatial data should be delivered in an ESRI useable format where applicable and documented with metadata in accordance with minimum BIOS metadata standards (http://bios.dfg.ca.gov/metadata.asp) and FGDC metadata standards (http://www.fgdc.gov/metadata/documents/workbook_0501_bmk.pdf).

d. SWRCB agrees to deliver high quality data in an acceptable and useable digital format for end users including summarizations of raw data; provide data updates as they become available; ensure all deliverable products contain metadata that meets the Federal Geographic Data Committee (FGDC) and BIOS standards; ensure all deliverables be submitted during the funding period; provide derived data products such as reports, summaries, graphs, and so on to the CDFW; and include the following verbiage in all contracts entered into by SWRCB: 
All data collected and created for this contract is a required deliverable of this contract and will become the property of the State of California, and not of the contracted party. A condition of final payment on this contract shall include the delivery of all related data. Spatial data should be delivered in an ESRI useable format where applicable and documented with metadata in accordance with minimum BIOS metadata standards (http://bios.dfg.ca.gov/metadata.asp) and FGDC metadata standards (http://www.fgdc.gov/metadata/documents/workbook_0501_bmk.pdf).

\section{Section 5. Funding}

a. All of the duties, roles and responsibilities specified herein are subject to the availability of funds to perform them.

b. The expenditure or advance of any money or the performance of any obligation of the State of California under this MOU will be contingent on appropriation and allotment of funds. No liability will accrue to the United States or the State of California in case funds are not appropriated or allotted.

c. Any future activities, including those involving funds or fiscal obligations, will be documented in an amendment to this agreement or in separate agreements referencing this agreement and signed by the parties, and should include a description of products or services to be provided, financial arrangements, legal authority citations, party responsibilities, duration of each project, and points of contact, subject to clearance by the Office of the Assistant General Counsel for Administration.

\section{Section 6. Dispute Resolution}

Should disagreements arise on the interpretation for the provisions of this agreement, or amendments and/or revisions thereto, that cannot be resolved at the operating level, the area(s) of disagreement shall be stated in writing by each party and presented to the other party for consideration. If agreement on interpretation is not reached within thirty days, the parties shall forward the written presentation of the disagreement to respective higher officials for appropriate resolution.

\section{Section 7. Effective Date and Termination}

This MOU may be executed in one or more separate counterparts. This MOU shall become effective when the counterparts have been executed by all the Parties hereto, and not before. Any Cooperator may terminate participation in this MOU on providing the other Parties 30 day written notice of intent to discontinue. 
THEREFORE, Cooperators, through their authorized representatives, approve and commit to implement the actions specifically described herein.

[CDFW Director Name]

California Department of Fish and Wildlife

DATE:

1416 Ninth Street, 12th Floor

Sacramento, CA 95814

[Agency Director Name]

California Department of [Agency Name]

DATE:

[Agency Address] 
Prepared by the Sacramento Publishing Service Center.

For more information concerning this report, contact:

Director, Pacific Region

U.S. Geological Survey

3020 State University Drive East

Modoc Hall, Suite 3005

Sacramento, CA 95819-2632

or visit our Web site at:

http://www.usgs.gov/saltonsea/ 
